\title{
THE PION CHARGE FORM FACTOR THROUGH PION ELECTROPRODUCTION
}

\author{
Tanja Horn
}

2006

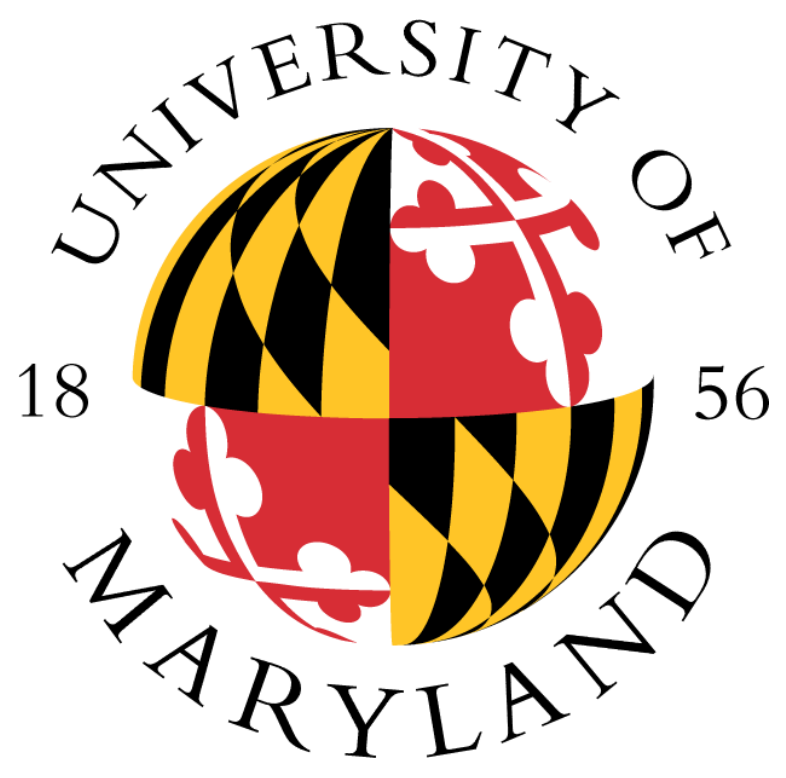





\begin{abstract}
Title of dissertation: THE PION CHARGE FORM FACTOR THROUGH PION ELECTROPRODUCTION

Tanja Horn, Doctor of Philosophy, 2006

Department of Physics
\end{abstract}

The goal of Jefferson Lab experiment E01-004 (F $\pi$-2) was the measurement of the longitudinal and transverse cross sections via pion electroproduction from hydrogen and deuterium for the purpose of extracting the charged pion form factor using pole dominance. The data were taken at two values of $Q^{2}(1.60$ and 2.45 $\left.(\mathrm{GeV} / \mathrm{c})^{2}\right)$. In order to attain full coverage in $\phi_{\pi}$, charged pions were detected in parallel kinematics (along the direction of momentum transfer, $q$ ), and at \pm 3 degrees off the direction of momentum transfer. For each $Q^{2}$ data were taken for two values of the virtual photon polarization, $\epsilon$, respectively. All data were taken at a fixed center of mass energy, $W=2.22 \mathrm{GeV}$. The longitudinal and transverse pieces of the cross section were separated using the Rosenbluth separation method. 


\title{
THE PION CHARGE FORM FACTOR THROUGH PION ELECTROPRODUCTION
}

\author{
by
}

Tanja Horn

Dissertation submitted to the Faculty of the Graduate School of the University of Maryland, College Park in partial fulfillment

of the requirements for the degree of

Doctor of Philosophy

2006

Advisory Commmittee:

Professor Elizabeth J. Beise, Chair/Advisor

Dr. David J. Gaskell

Professor William Walters

Professor Philip G. Roos

Professor Thomas D. Cohen

Professor Chia-Cheh Chang 
(c) Copyright by

\section{Tanja Horn}

2006 


\section{ACKNOWLEDGMENTS}

I am indebted to many people who have made it possible for me to complete this Ph.D. dissertation. First, I want to thank my academic advisor, Betsy Beise, who has always been a supportive friend and a truly respectful person. Over the years, Betsy has given me freedom and trust to work on my own, but she has always taken time out of her busy schedule whenever guidance was needed.

My special regards go to Dave Gaskell, who gave me confidence, trust and support to work on this challenging and rewarding project for my dissertation. Dave worked with me on several difficult analysis problems, and always inspired me with new ideas when I got stuck. Dave also taught me about the Moller polarimeter in Hall $\mathrm{C}$ and gave me the opportunity to work on refurbishing the Moller scintillators. I respect Dave as an honest and unbiased physicist from whom I have learned not only physics but also the right attitude towards experimental research.

I have to thank the Physics Department at the University of Maryland, in particular, the experimental nuclear physics group: George Chang, Phil Roos, Jim Kelly, Herbert Breuer and Nick Chant. I am grateful to George for recruiting me into the group during my undergraduate studies and getting me started in experimental nuclear physics in Hall A at Jlab. I thank our group secretary, Judy Myrick, who always keeps everything organized. I am privileged to have my years in the group overlapping with former and present graduate students: Kenneth Gustafsson, 
Damon Spayde, Nikolai Savvinov, Jianglai Liu, Chad Galley, Colleen Ellis and Jon Miller, and undergraduates Lance Clevenger, Mary Claire Herda, Kristin Kiriluk, Ken Rossato, Erick Andrade and Andrew Parker.

During my time at the University of Maryland I worked with many fantastic people. I like to thank Chad Galley, Willie Merrell, Nick Cummings and Ahmad Idilbi for helping me understand various theoretical concepts throughout my graduate studies and moral support during my thesis defense. In particular, I like to thank Chad Galley for many long night-time discussions on non-equilibrium dynamics. My regards go to Dan and Beth Dakin for many physics discussions and helping me to organize the quantum field theory class. I also want to thank Ira Thorpe for sitting through endless study sessions and taking all first year classes with me and Ray Hodges for listening to all my explanations while studying for the second round of the qualifier. I am grateful to professor Bei-Lok Hu for teaching me the applications of gravitational theory to other research and for helping me on the quark gluon plasma project.

My time as a student at Jefferson Lab has been both educational and interesting. I thank all people at the lab I interacted with directly or indirectly. In particular, the Hall C scientists under the leadership of Rolf Ent: Steve Wood for helping me with the computers, Mark Jones for helping me to figure out several difficult spectrometer optics problems, Howard Fenker for discussions on scintillators and electronics, Dave Mack for giving me the opportunity to work on this experiment, Greg Smith for teaching me about cryogenic targets, Bill Vulcan for helping me with photomultiplier tubes and electronics, Dave Meekins for many scientific 
conversations, Dave Gaskell for enduring even my most irrelevant questions, reading my thesis front to back once and serving on my thesis committee, and Rolf Ent for advice and support on spectrometer optics problems and allowing me to sit in his Nuclear Physics class. I also want to mention all the other people at JLab who kept this experiment running. The target group under the leadership of Mike Seeley for making sure that we had cold targets, the Yerevan group under the leadership of Hamlet Mkrtchyan for building the aerogel cherenkov detector, and the engineering and technical staff - Paul Brindza, Mike Fowler, Steve Lassiter, Walter Kellner and his crew, Joe Beaufait and many others. I thank Eric Christy for discussions on kinematic offsets, optics and tracking. My regards go to John Arrington for scientific advice, feedback on parts of my thesis and helping me with some of the time of flight calibration issues.

I thank the entire $\mathrm{F}_{\pi}-2$ collaboration. In particular, the spokespeople, Henk Blok, Dave Mack and Garth Huber for their trust and support and getting this experiment approved and running. I thank Henk Blok for working with me on many difficult spectrometer optics problems, Garth Huber for many theoretical discussions, help and advice, and Dave Mack for getting me started when I first came to Hall $\mathrm{C}$ and for working with me on important parts of the analysis. I want to thank Jochen Volmer for helping out and taking so many night shifts on this experiment. Jochen's hard work on $\mathrm{F}_{\pi^{-1}}$ played an integral part in the analysis of the $\mathrm{F}_{\pi}-2$ data. I acknowledge my fellow students and postdocs at JLab: Ben Clasie, Leon Cole, Aji Daniel, Nadia Fomin, Sabine Fuchs, Narbe Kalantarians, Ya Li, Simona Malace, Peter Monaghan, Itaru Nakagawa, Jason Seely, Patricia Solvignon and 
Vince Sulkosky, the G0 students and postdocs: Jianglai Liu, Sarah Phillips, Kaz Nakahara, Silviu Covrig, Lars Hannelius, Mat Muether, Colleen Ellis, and Stephanie Bailey, Fatiha Benmokthar, Michael Gericke, Julie Roche and Paul King, and many others. Thanks to Ameya Kolarkar for always listening and helping me to figure out how to make posters (and black boxes) in Open Office. Special thanks to Victor Rodriguez for reading parts of my thesis.

Throughout my graduate studies I enjoyed the support of friends and family. I have to mention three people without whom I would not have reached this milestone. First of all I thank Brett Bassett for always supporting me although we never seem to be in the same place at the same time. I thank Dave Walker for giving me confidence that I could succeed in physics - really my turning point from chemistry, and Nick Chant for trusting that I could succeed in the Physics graduate program at UMd.

I acknowledge all my defense committee members: Betsy Beise, Dave Gaskell, William Walters, Phil Roos, Tom Cohen, and George Chang for taking the time reading this thesis. I particularly appreciate Betsy and Dave's efforts of turning this lengthy manuscript full of language mistakes into a useful document. This work was supported in part by the U.S. Department of Energy and research grants from the U.S. National Science Foundation 


\section{TABLE OF CONTENTS}

List of Tables vii

List of Figures viii

1 Introduction 1

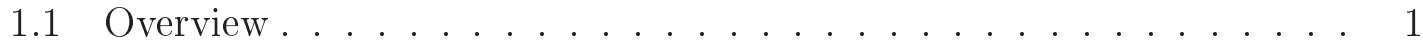

1.2 The Pion Form Factor in the Asymptotic Limit . . . . . . . . . . 8

1.3 Pion Form Factor Dynamics . . . . . . . . . . . . . . . 13

1.3.1 QCD Sum Rules .................. . . 14

1.3.2 Lattice Calculations . . . . . . . . . . . 15

1.3 .3 Models ....................... 17

1.4 Pion Electroproduction . . . . . . . . . . . . . . . 25

1.4.1 Kinematics Definition . . . . . . . . . 25

1.4.2 Cross Section Definition ............. 28

1.4.3 Pion Electroproduction Models ............ . 30

1.4.4 Competing Reaction Channels . . . . . . . . . . . . 35

1.5 Overview of the Experiment . . . . . . . . . . . . 37

2 Experimental Apparatus 39

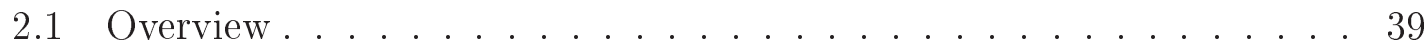

2.2 Accelerator . . . . . . . . . . . . . . . 39

2.3 Hall C . . . . . . . . . . . . . . . . . . 41

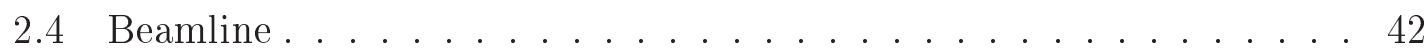

2.4.1 Beam Position Monitors . . . . . . . . . . . . . 42

2.4.2 Beam Energy Measurement . . . . . . . . . . . 44

2.4.3 Beam Current Monitors ............... . . 46

2.4.4 Modification to Beamline . . . . . . . . . . . 48

2.5 Targets . . . . . . . . . . . . . . . . . . 49

2.5.1 Cryogenic Targets: Hydrogen and Deuterium . . . . . . . . 50

2.6 Target Thickness . . . . . . . . . . . . . . 51

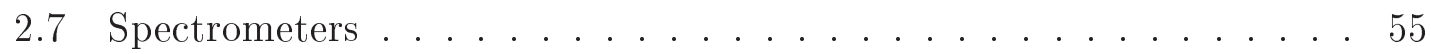

2.7.1 High Momentum Spectrometer (HMS) ......... 55

2.7.2 Short Orbit Spectrometer (SOS) . . . . . . . . 60

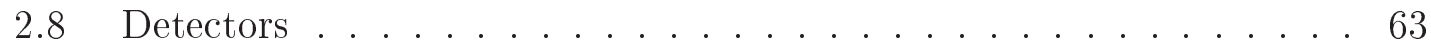

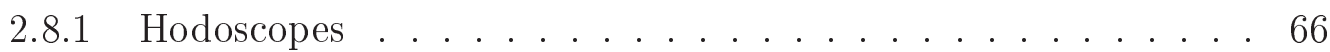

2.8.2 Čerenkov Detectors . . . . . . . . . . . . . 68

2.8.3 Lead Glass Calorimeter . . . . . . . . . . . . . . 69

2.8.4 HMS Aerogel Čerenkov Detector ........... . 70

2.9 Trigger System and Data Acquisition . . . . . . . . . . . . . 74

2.9.1 HMS Pretrigger ................... 74

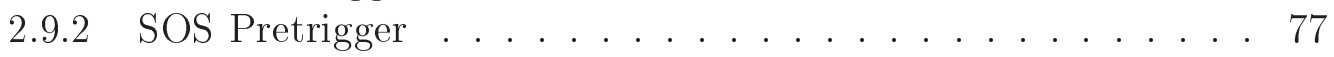

2.9.3 Coincidence Trigger . . . . . . . . . . . . . 78

2.10 Data Acquisition . . . . . . . . . . . . . 80 
3 Data Analysis $\quad 82$

3.1 Tracking and Reconstruction . . . . . . . . . . . . . . 82

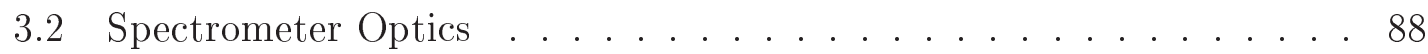

3.2.1 Spectrometer Optics for Extended Targets in $y_{t a r}$. . . . . . 91

3.2.2 Spectrometer Acceptance . . . . . . . . . . . . . . 95

3.3 Experimental Offsets . . . . . . . . . . . . . . 100

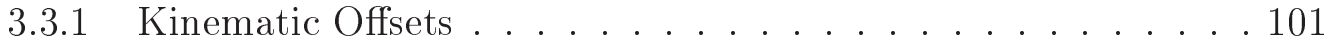

3.3.2 Fast Raster Correction . . . . . . . . . . . . . . 107

3.4 Particle Identification and Event Selection . . . . . . . . . . 109

3.4.1 Time of Flight . . . . . . . . . . . . . . . . . 109

3.4.2 Electron Identification . . . . . . . . . . . . . 110

3.4.3 Pion Identification . . . . . . . . . . . . . . . . . . . . . . . . . . . . . . . 114

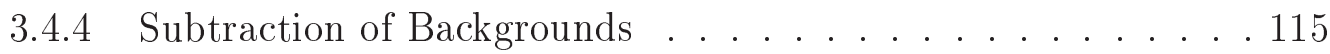

3.5 Efficiency Corrections . . . . . . . . . . . . . . . 120

3.5.1 Trigger Efficiency . . . . . . . . . . . . . . 120

3.5.2 Tracking Efficiency and Multiple Tracks . . . . . . . . . 122

3.5.3 Computer and Electronic Deadtimes . . . . . . . . . . 129

3.6 Coincidence Blocking . . . . . . . . . . . . . . . 138

3.7 Čerenkov Blocking . . . . . . . . . . . . . . . . . 141

3.8 Pion Absorption in the HMS . . . . . . . . . . . 146

4 Simulation $\quad 152$

4.1 Monte Carlo Overview . . . . . . . . . . . . . . . . . 152

4.2 Event Generation . . . . . . . . . . . . . . . 153

4.3 Spectrometer Models . . . . . . . . . . . . . . . 155

4.4 Passage Through Materials . . . . . . . . . . . . . . . 157

4.4.1 Ionization Energy Loss . . . . . . . . . . . . . . . 158

4.4 .2 Multiple Scattering . . . . . . . . . . . . . 159

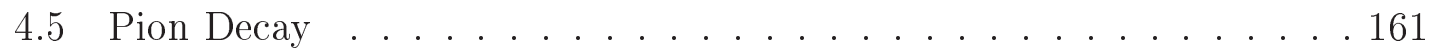

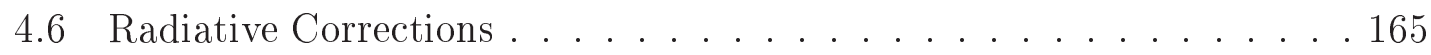

4.7 Elastic Scattering . . . . . . . . . . . . . . . 174

5 Experimental Cross Sections Overview 180

5.1 Monte Carlo Equivalent Yield . . . . . . . . . . . . . . . 182

5.2 Determining Cross Sections . . . . . . . . . . . . . . 184

5.3 Model Cross Section . . . . . . . . . . . . . . 186

5.4 Comparing Data and Monte Carlo . . . . . . . . . . 187

5.4.1 Iteration of the Input Model Cross Section . . . . . . . . . 188

5.4.2 Cross Section Model Dependence . . . . . . . . . . . . 193

5.5 Cross Section Spectrometer Acceptance Dependence . . . . . . . . 196

5.6 Error Analysis . . . . . . . . . . . . . . . 199 
6 Results and Discussion 203

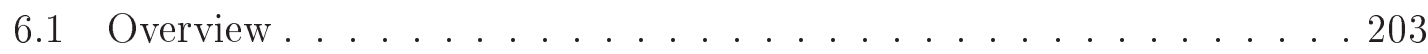

6.2 Experimental Cross Sections . . . . . . . . . . . . . . 204

6.3 Unseparated Cross Sections . . . . . . . . . . . . 205

6.4 Separated Cross Sections . . . . . . . . . . . . . . 206

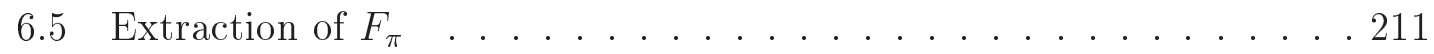

$6.6 \pi^{-} / \pi^{+}$Ratio and Pole Dominance . . . . . . . . . . . . 214

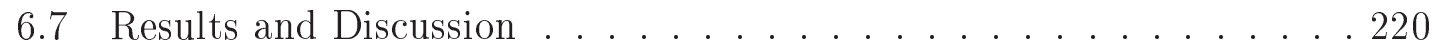

6.8 Future Measurements . . . . . . . . . . . . . . . . . . . . . . . . . . . . . . . . . . . 222

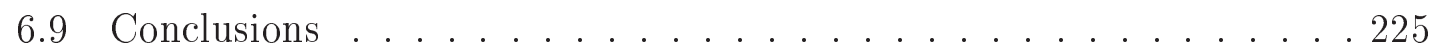

A Target Density - Luminosity Scan Runs 227

B Target - Thermal Contraction 237

$\begin{array}{ll}\text { Bibliography } & 240\end{array}$ 


\section{LIST OF TABLES}

1.1 Kinematic settings measured in $F_{\pi}-1$ and $F_{\pi}-2 . \ldots . \ldots . . . . .38$

2.1 Cryotarget thicknesses, not corrected for beam offset. The cryotarget length, measured at room temperature, is corrected for thermal contraction of the aluminum cell walls and the offset of the cryotarget from the surveyed target position $(3.42 \mathrm{~mm})$. The final target length is given by $L=2 \cdot \sqrt{R^{2}-d x^{2}}$, where $R$ is the target cell radius corrected for thermal contraction and with the cell walls subtracted, and $d x$ is the beam offset from the target center. The actual target thicknesses for these targets were also corrected for the beam offset from the target center at each kinematic setting (see Figure ??). The uncertainty on the target thickness is the quadrature sum of $0.6 \%$ uncertainty on the target length and $0.5 \%$ on the target density. . . . . . . . . . 52

2.2 Cryotarget thickness uncertainties. The random uncertainty is determined by the beam position on target. . . . . . . . . . . . . . . 54

3.1 Experimental settings for the $F_{\pi}$-2 elastic coincidence runs. Electrons were detected in the SOS and protons were detected in the HMS. . . . 102

3.2 Kinematic offsets measured in $F_{\pi}$-2. . . . . . . . . . . . 107

3.3 SOS Čerenkov and SOS calorimeter cut efficiencies. The electron cuts were $N_{\text {photoelectrons }}>0.5$ and $E_{\text {calorimeter }} / E^{\prime}>0.7$ for $F_{\pi}$-2. . . . 113

3.4 HMS Čerenkov blocking correction at high and low $\epsilon$. The values shown are for representative runs at each setting. . . . . . . . . . . . 144

3.5 Pion absorption correction and associated uncertainties. . . . . . . . 149

3.6 Materials in HMS and SOS contributing to absorption and multiple scattering as particles traverse the target and spectrometers. . . . . . . 151

4.1 Comparison of data to Monte Carlo as a function of missing mass $\left(M_{x}\right)$ cut in the radiative tail. The ratios are normalized to 1.0 at the largest missing mass cut. Note that the region above $1.02 \mathrm{GeV}$ up to two pion threshold is excluded to avoid contamination of the sample from collimator punchthrough events. . . . . . . . . . . . 171

5.1 Fit parameters for equation ?? before and after iterating the model. Convergence was achieved within 2 iterations. . . . . . . . . . . . . 194 
5.2 Summary of systematic uncertainties. The uncertainties listed correspond to the uncertainty in the cross section as a result of the uncertainty in the correction. Point to point uncertainites are applied at each $\epsilon$ point when calculating the separated cross sections, $\sigma_{L}$ and $\sigma_{T}$. Scale uncertatinties are directly associated to the separated cross sections. The values in the "Correlated (-t)" column denote uncertainties that change the t-dependence of the high and low epsilon dependent cross sections in a correlated way. These uncertainties are applied directly to $F_{\pi}$ after extracting $\Lambda_{\pi}^{2}$. Adding all uncertainties in quadrature results in a total point to point uncertainty of $1.2(0.9) \%$ and a total scale uncertainty of 3.5\%. The partially correlated uncertainty ranges between $1.8 \%$ and $1.9 \%$. . . . . . . . . . . . . 202

6.1 Unseparated cross sections, $\frac{d \sigma}{d t}$, for the $\left(e, e^{\prime}, \pi^{+}\right)$reaction. The results are presented in the lab frame for the center value of each $t$ bin. The values for $W$ and $Q^{2}$ are the weighted averages for each $t$ bin averaged over the high and low $\epsilon$ points. The values for $\epsilon$ and $\theta^{*}$ are calculated from $W, Q^{2}$ and $t$ in each such bin and the corresponding beam energies for the $\epsilon$ calculation. The uncertainties shown are statistical and random systematic combined in quadrature. Note that random systematic uncertainties have to be included at each $\epsilon$ point using equation ??, while scale systematic uncertainties propagate directly into the separated cross sections. . . . . . . . . . . . . . . 206

6.2 Separated cross sections for the $\left(e, e^{\prime}, \pi^{+}\right)$reaction for both values of $Q^{2}$. The errors on the cross sections are statistical and systematic, while the errors on the ratios are the total errors. . . . . . . . . 208

6.3 Values for $\Lambda_{\pi}^{2}$ and $F_{\pi}$. The values for $\Lambda_{\pi}^{2}$ are extracted from a comparison of the separated longitudinal cross section to the one from the $V G L /$ Regge model prediction and $F_{\pi}$ is determined using equation ??. Note that these values are dependent on the particular model used. . . 213

6.4 Longitudinal separated ratios cross section ratios. Statistical and total systematic uncertainties are shown. The cross sections have been evaluated using the hydrogen model. . . . . . . . . . . . . . 220

A.1 Kinematic settings for the $F_{\pi}$-2 luminosity scans. . . . . . . . . 228

B.1 Material properties for Aluminium alloys used in Hall C target cell

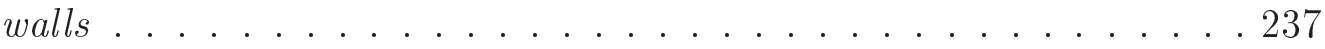




\section{LIST OF FIGURES}

1.1 $F_{\pi}$ elastic $\pi$-e data from CERN and pion electroproduction data from CEA, Cornell III, DESY. The error bars are statistical only. Also shown are $F_{\pi}$ pion electroproduction $L / T$ data from JLab Hall C $\left(F_{\pi^{-}}\right.$ 1). The solid curve is a monopole fit to the CERN data $\left(r_{\pi}=0.662\right.$ fm, Ref. [4]). The dashed curve is a monopole fit to the Cornell III analysis result $\left(r_{\pi}=0.711 \mathrm{fm}\right.$, Ref. [6]). . . . . . . . . .

1.2 $F_{\pi}$ in hard scattering in the valence quark picture to lowest order. The photon couples to a quark with subsequent gluon exchange. The quantities $x$ and $y$ indicate the momentum fractions carried by individual valence quarks in the inital and final state respectively. . . . . . . .

1.3 $F_{\pi}$ theoretical predictions from a constituent quark model (solid line, Ref. [22]), QCD sum rules (long dashed, Ref. [23]), Bethe-Salpeter/ Dyson-Schwinger equations (short-dashed, Ref. [24]) and next to leading order perturbative QCD with and without estimated soft contributions (dashed-dotted, Ref. [26]). The $F_{\pi}$ data are as in Figure 1.1. . . 11

1.4 Lattice calculation of the pion form factor by Bonnet et al. [37]. The calculation uses a chiral-symmetry preserving domain wall action and lattice pion masses of $318 \mathrm{MeV}$ and $758 \mathrm{MeV}$. A domain wall on the lattice can be viewed as a plane separating degenerate solutions to the Dirac equation. The shaded regions indicate the uncertainty for a vector meson dominace fit. Note that no chiral extrapolation has been done in the lattice results shown in the figure. The datapoints shown are from $F_{\pi}-1 \ldots \ldots \ldots \ldots$

1.5 Local Duality prediction of the pion form factor using Fermilab E615 Drell-Yan data on the pion structure function. The leading order analysis of the experimental data was used in the calculation. The two dashed lines denote linear and quadratic $x$-dependeces. The dotted line is the asymptotic leading order $p Q C D$ prediction. The figure is

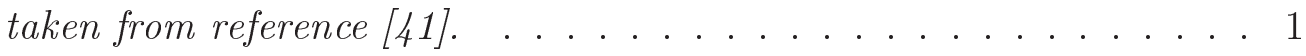

1.6 Diagram of the Bethe-Salpeter equation. Figure from reference [46]. . 20 
1.7 Impulse approximation for the pion form factor. The model amplitude for the mesons are the solutions of the homogeneuos Bethe-Salpeter equation, which has two inputs: 1) Quark propagator and 2) the scattering kernel. Integrating over one of the variables in the path integral containing all orders one arrives at a hierachy of coupled n-point functions. The calculation is usually performed in a Euclidean metric in momentum-space, which is the Fourier transform of the lattice

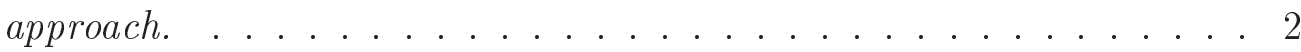

1.8 Diagram of transition of the physical pion to a pair of valence quarks separated on the lightcone. This process is described by the pion dis-

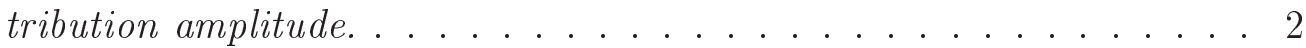

1.9 Consituent quark model predictions for the pion form factor compared to $Q C D$ sum rule and dispersion relation calculations. Shown are a constituent quark model calculation by Cardarelli (dashed-dotted, [22]), a more recent constituent quark analysis with different confining potentials (solid, [53]), QCD sum rules (dotted, [23]), and a dispersion relation calculation by Geshkenbein (dashed, [54]). The dispersion relation approach relates timelike and spacelike regions of the pion form factor in the complex plane and is generally rigorous. However, the approach is not well constrained in the timelike region. . 24

1.10 Schematic representation of the pion electroproduction process. The three-vectors of the initial and final electron define the scattering plane. The momentum transferred to the photon-nucleon system is defined in terms of the four-momentum transfer, $Q^{2}$. The reaction plane is defined by the three-momentum vectors of the pion and the recoiling nucleon. The pion is emitted at an angle $\theta_{\pi}$ relative to the virtual photon direction and the angle between the scattering and reaction plane is $\phi_{\pi}$. Figure from reference [56] . . . . . . . . .

1.11 Polar plot of the $-t$ phase space. The radial component is $-t$ shown as two circles for values of $-t=0.1$ and $0.3(\mathrm{GeV} / \mathrm{c})^{2}$. The angular component is $\phi_{\pi}$ which progresses counter-clockwise with $\phi_{\pi}=0$ to the right. The full $\phi$ coverage is obtained for the high $\epsilon$ setting at three HMS central angles $(0, \pm 3) \ldots \ldots . \ldots \ldots$

1.12 Born term diagrams for pion electroproduction. (a) t-channel (pion pole) mechanism, (b) s-channel (nucleon pole), (c) u-channel (crossed nucleon pole), (d) contact term required to restore gauge invariance.

1.13 Longitudinal and transverse cross sections calculated in the VGL Regge model for different values of $\Lambda_{\rho}^{2}$. While the longitudinal term is not affected at low -t, the transverse term changes significantly. . . . . . . 34 
1.14 Pion electroproduction cross section calculations for $F_{\pi}$-2 kinematics from the VGL/Regge model ([11] and a quark model prediction by Obukhovsky et al. [58]. Both models provide a similar good description of the expected $t$-dependence of the longitudinal cross section for $\Lambda_{\pi}^{2}=0.54(\mathrm{GeV} / \mathrm{c})^{2}$. The quark model prediction for the transverse piece of the cross section exceeds the VGL/Regge prediction by a fac-

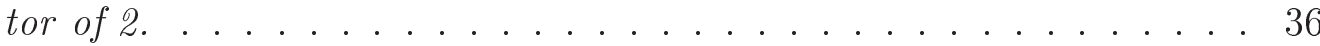

2.1 Schematic representation of the JLab site. . . . . . . . . . . 40

2.2 Schematic representation of the JLab accelerator. . . . . . . . . 42

2.3 Schematic representation of the instrumentation along the Hall $C$ beamline..................... 43

2.4 Beam Line Elements. . . . . . . . . . . . . . . . . . . . . . 45

2.5 Cross section of the cryotarget loop. ............... 52

2.6 Beam position offset with respect to cryotarget center. The vertical axis denotes the location of the cryotarget along the beam in the lab frame. The horizontal axis shows the position of the beam relative to the target center also in the lab frame. ........... 53

2.7 Schematic top view of the Hall $C$ spectrometers relative to the target

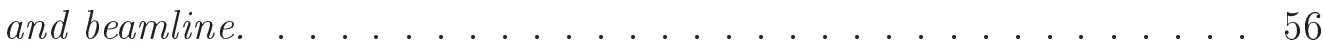

2.8 Schematic representation of the HMS. The spectrometer consists of three focusing quadrupole magnets and one vertical bend dipole magnet. The distance from the target to the focal plane is $\approx 26 \mathrm{~m} . . .55$

2.9 Schematic sideview of the HMS detectors. The lead glass calorimeter is tilted as an angle of $5^{\circ}$ relative to the central ray to minimize the loss of particles through the spaces between the calorimeter blocks. . . 5

$2.10 B_{Q 3} / P_{H M S}$ normalized to 1.0 at $P_{H M S}=2.77 \mathrm{GeV} / \mathrm{c}$. In the ideal case, the ratio is expected to be constant as a function of HMS central momentum. The 1998 data were obtained from reference [71]. The data from 2003 are separated into time periods before and after the Hall probes were zeroed. . . . . . . . . . . . . 59

2.11 Schematic sideview of the SOS. The distance from the target to the SOS focal plane is $\approx 10 \mathrm{~m}$. Figure from reference [92]....... 61 
2.12 SOS central momentum Saturation correction. The SOS central momentum offset found for $F_{\pi}-1$ result in a true value for the central momentum about $0.45 \%$ higher than the nominal set point. This offset has been removed in the figure in order to compare the $F_{\pi}$-1 data points to the ones from $F_{\pi}$-2. The 2004 data points are from

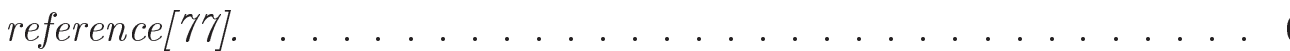

2.13 Representative SOS Čerenkov and SOS Calorimeter spectra. On average there are 7 photoelectrons per electron for the SOS Čerenkov with little position dependence for three of the four mirrors. Due to localized inefficiency in one of the mirrors (\#2), a low photoelectron cut $\left(N_{\text {photoelectron }}>0.5\right.$ was used. The calorimeter provides additional particle identification and eliminates residual real $\pi^{+} / \pi^{-}$coincidences. The arrow indicates the cut applied to select electrons. . . .

2.14 Schematic drawing of the HMS Aerogel Čerenkov detector. The detector was installed in the HMS in 2003 to provide more efficient particle identification at spectrometer central momenta above $3 \mathrm{GeV} / \mathrm{c}$. Two configurations with refractive indeces 1.03 and 1.015 are available. For $F_{\pi}$-2 aerogel with $n=1.03$ was used to reject protons. . . . . . . 72

2.15 Aerogel Čerenkov Detector. The mean number of photoelectrons $\left(N_{p e}=\right.$ 12) is stable up to particle rates of $600 \mathrm{KHz}$. The arrow indicates the cut used in this analysis. . . . . . . . . . . . . . . 73

2.16 Schematic of spectrometer pretrigger logic. There are four options at the pretrigger module: 1) ELREAL standard electron trigger, 2) PIPRE prescaled 3/4 pion trigger, 3) SCIN 3/4, and 4) PIONHI 3/4 with Čerenkov veto. Option 4 was added in the HMS trigger logic

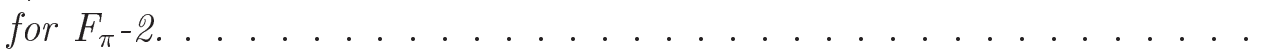

2.17 Schematic of spectrometer pretrigger logic. There are four options at the pretrigger module: 1) ELREAL standard electron trigger, 2) PIPRE prescaled 3/4 pion trigger, 3) SCIN 3/4, and 4) PIONHI $3 / 4$ with Ćerenkov veto. Option 4 was added in the HMS trigger logic for $F_{\pi}$-2. . . . . . . . . . . . . . .

3.1 Reconstructed invariant mass, $W$, from an elastic (e, $\left.e^{\prime}\right)$ delta scan as a function of $H M S x_{f p}^{\prime}$ at $P_{H M S}=4.005 \mathrm{GeV} / \mathrm{c}$. If the matrix elements are correct, $W$ is expected to reconstruct to the proton mass independent of target and focal plane quantities. With no further correction, the centroid of the elastic peak varies by 2-3 MeV across the $x_{f p}^{\prime}$ acceptance. Applying an ad-hoc $x_{f p}^{\prime}$ correction to the reconstruction of the data eliminates most of the correlation. . . . . . . . . . . . 86 
3.2 HMS $\left(\delta, x_{f p}^{\prime}\right)$ correction $\left(\epsilon_{\text {corr }}\right)$ as a function of HMS central momentum. This correction is applied to all data in the $F_{\pi}$-2 analysis. The 2004 data were obtained from reference [71]. . . . . . . . . . . 8

3.3 Reconstruction of the HMS sieve hole pattern. The solid line denotes the acceptance defined by the octagonal collimator. Note that the sieve holes in the figure are denoted by ellipses, while the collimator sieve holes are circular. . . . . . . . . . . . . . . .

3.4 Difference between the reconstructed out of plane and the value calculated from sieve hole location and geometry, not corrected for horizontal beam position, for the outermost quintar targets at $z=-7.11,+7.91$ $\mathrm{cm}$. The symbols represent the 9 vertical columns of the sieve collimator. The difference between reconstructed and calculated $x_{\text {tar }}^{\prime}$ varies by 4.5 mrad across the acceptance. This slope may be attributed to a modification in the out-of-plane angle, $x_{\text {tar }}^{\prime}$, resulting in a nonzero vertical beam position in the optical plane of the spectrometer, which in turn affects the reconstuction of $x_{\text {tar }}^{\prime}$. The sign of the slope depends on the target position along the beam, since $x_{t a r}^{\prime}=\frac{d x}{d z} . \ldots \ldots$

3.5 Diagram of the target in spectrometer coordinates. The angle is different at extreme target positions relative to $z=0$ resulting in a vertical beam position offset in the optical plane. Note that the sign of $x_{t a r}^{\prime}=-\frac{x_{t a r}}{z_{t a r}}$ changes with the sign of $z_{\text {tar }}$, since the scattering angle is always defined to be positive. . . . . . . . . . . . . . .

3.6 HMS $x_{f p}^{\prime}$ vs $x_{f p}$ for the two outermost quintar targets. The angle for the outermost sieve holes varies by $\approx 7$ mrad for foils at $z=+7.91$ $(-7.11) \mathrm{cm}$ relative to the one at $z=0$. The change in angle leads to a non-zero vertical beam position in the HMS optical plane $\left(x_{t a r} \neq 0\right)$, which in turn affects the reconstructed variables. . . . . . . . . . .

3.7 HMS $y_{\text {tar }}^{\prime}$ acceptance for elastic $e-p$ data at $P_{H M S}=3.83 \mathrm{GeV} / \mathrm{c}$ and $\theta_{H M S}=12^{\circ}$. The left panel shows the distribution of data (crosses) and SIMC (solid line). The acceptance is generally described well except for small regions at the edges. While this discrepancy is likely of no major concern if one integrates over this region, it is important in this analysis due to the direct correlation with $-t . \ldots 96$ 
3.8 Reconstructed missing mass from $H\left(e, e^{\prime} \pi^{+}\right) n$ data plotted vs. HMS $y_{f p}^{\prime}$ (hsypfp) at $P_{H M S}=2.93 \mathrm{GeV} / \mathrm{c}$. The missing mass should reconstruct to the neutron mass independent of target and focal plane variables. However, the missing mass centroid varies by $1.5 \mathrm{MeV}$ across the hsypfp acceptance. A similar effect is observed in elastic data, where the invariant mass is studied as a function of hsypfp. To eliminate this correlation an ad-hoc correction is fit using $H\left(e, e^{\prime} \pi^{+}\right) n$ data (see text). . . . . . . . . . . . . . 99

$3.9 \operatorname{HMS}\left(\delta\right.$, hsypfp) correction $\left(C_{\delta}\right)$ as a function of HMS xpfp. . . . 100

3.10 HMS central angle offset study using ep elastic data. The difference between invariant mass and proton mass is shown as a function of HMS angle for two sets of kinematic offsets. The linear correlation at forward angles in the upper panel was determined to be partially due to a 1 mrad offset in the HMS central angle. However, to completely eliminate the correlation, the $x_{f}^{\prime} p$ correction to HMS $\delta$ (see section ?? has to be taken into account as well. . . . . . . . . . . . . . . . 103

3.11 Out of plane Offsets determined from elastic coincidence data. The fraction of electron and proton momentum is calculated from the spectrometer central momenta. The values on the vertical axis include the offset of the vertical beam position at each kinematic setting. The vertical beam position is determined from sieve slit data and BPM information in this analysis. . . . . . . . . . . . . . 106

3.12 Fast raster $Y$ position (ie. vertical beam position) as a function of missing energy for elastic coincidence data before and after correcting the amplitude. . . . . . . . . . . . . . . 108

3.13 Position at the HMS S2Y hodoscope plane as a function of $\beta_{T O F}$. The hodoscope resolution is affected by erratic behavior of at least three paddles in the center of the plane. The resolution could be improved by eliminating a large fraction of the PMTs on one side of the plane from the time of flight calculation. The PMT signals were included on the trigger level, so that no good events were lost. . . . . . . . . . 111

3.14 SOS Čerenkov efficiency. . . . . . . . . . . . . . . 113

3.15 HMS aerogel Čerenkov detector horizontal acceptance and detector cut efficiency as a function of horizontal acceptance for three different photoelectron cuts. The cut efficiency for the cut used in this analysis

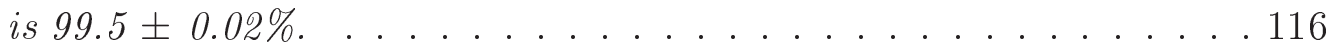


$3.16 F_{\pi}$-2 coincidence time spectrum as a function of $\beta_{T O F}-\beta_{p}$ including the real (solid) and random (dashed) coincidence time cuts. Real $e-\pi$ coincidences can be clearly identified and real proton coincidences are rejected by the coincidence time cut. The random coincidence background is $<1 \%$ for all kinematic settings. The "tail" in the time of flight spectrum at low $\beta$ - $\beta_{p}$ results from pions interacting hadronically in the scintillator paddles. These events were eliminated to simplify the coincidence time cut. These lost "good" events must be accounted for in the efficiency calculation. . . . . . . . . . . . . . . 117

3.17 HMS trigger efficiency from elastic $\left(e, e^{\prime}\right)$ data. The upper panel shows the acceptance for electron kinematics with central $\delta$ centered at $-4 \%$. The lower panel shows the predicted 3/4 trigger efficiency using the algorithm described in the text. At negative $\delta$ the data indicate a significant ineffciency which may be attributed to scintillator paddle performance. This inefficiency is of no significant concern in the $F_{\pi}-2$ data analysis, since the HMS acceptance is limited from $-5 \%$

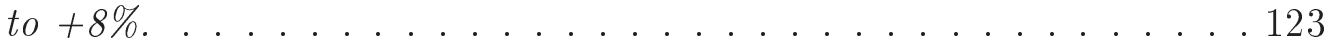

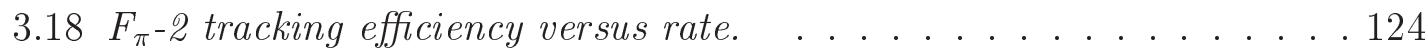

3.19 Multiple track contribution to electron data. The upper panel shows a distribution of the total energy deposited in the calorimeter. Nominally, electrons deposit all energy and appear at unity (normalized). Two electron clearly contribute in the region above 1.0 and the contribution changes as a function of rate. The solid line shows data taken at a rate of $400 \mathrm{KHz}$ and the dashed line indicates data taken at a rate of $700 \mathrm{KHz}$. The lower panel shows the multiple track background as a function of rate calculated from equation ??. . . . . . . . . . 126

3.20 HMS tracking efficiency for inelastic electron data taken during luminosity scans. The open symbols indicate the nominal tracking efficiency calculation neglecting multiple track contribution and the filled symbols represent the calculation correcting for multiple tracks. The solid line indicates the prediction of for the HMS tracking efficiency using equation ?. . . . . . . . . . . . . . . . . . 128

3.21 Schematic of the HMS detectors in one event display mode. Shown is an electron trigger for which multiple tracks were found. The red line indicates the track selected by the tracking algorithm and the remaining cluster of events are failed events. . . . . . . . . . . 129 
3.22 Efficiency corrected and charge normalized yields for data runs taken at constant current, but at varying computer deadtime. The error bars shown are statistical only. The solid and dashed lines indicate the average yield and the statistical deviation from it. While the computer dead time correction varies by $40 \%$ between runs, the yields agree to

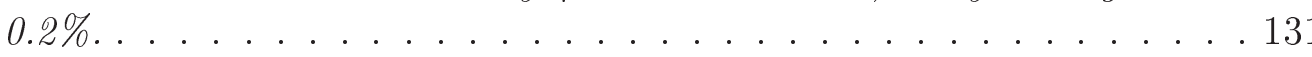

3.23 Electronic live time as a function of pretrigger rate into HMS and SOS. The data are fit to equation ??. The data shown are taken with no particle identification on the trigger level. . . . . . . . . . . . . 139

3.24 HMS Coincidence Time Spectrum. The main coincidence time window is centered around 1700 TDC channels, while the region to the left are early singles events in the SOS. Due to the specific coincidence time trigger conditions no early events from HMS singles are present. The conversion to nanoseconds is 0.1 TDC channels/ns. . . . . . . 140

3.25 Measured SOS coincidence blocking correction due to SOS early singles as a function of pretrigger rate. The curves shown are the fit results to the data from equation ?? with the value of $\tau$. . . . . . . . . 142

3.26 HMS Čerenkov Blocking TDC spectrum for electron events as identified by the HMS Čerenkov ADC. The central peak corresponds to signal from the electrons that result in the trigger. The second peak is due to a second electron arriving within the timing window. The backgrounds to the left and right of the two peaks are due to earlier and later electrons. . . . . . . . . . . . . . . . . . 143

3.27 Čerenkov blocking as a function of electron rate in the HMS. The solid line is a fit to equation ??. The fit value for $\tau$ is larger than the measured Čerenkov TDC gate width ( $\approx 100$ ns, dashed line). This discrepancy may be attributed to the width of the Čerenkov signal. While the ADC gate is fixed, the Čerenkov signal itself has some width and the overlap determines an effective gate width. . . . . . . . 145

3.28 Parametrization of the $A$ dependence of the $\pi-A$ cross section. The

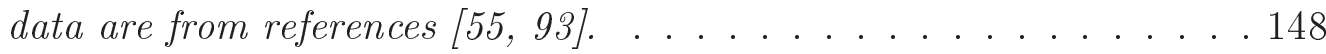

4.1 Comparison of data (crosses) and SIMC (solid line) charge normalized counts for HMS reconstructed quantities. The fractional momentum hsdelta and the spectrometer angles hsxptar and hsyptar are used in the calculation of the relevant physics variables. Generally, the Monte Carlo describes the data quite well except for small regions at the edges of the hsyptar acceptance. A similar effect was observed in elastic electron data. . . . . . . . . . . . . . . . 156 
4.2 Simulated HMS $Y_{\text {tar }}^{\prime}$ acceptance for a point target for different spectrometer exit window thicknesses. The HMS matrix elements were fit using a window made of Kevlar/Mylar $\left(X_{0}=53.3\right)$, while the exit window used in this experiment is made of Titanium $\left(X_{0}=3.56\right)$. Multiple scattering is present in all three cases, but has increased in the case of the thicker Titanium window at low HMS momenta. The size of the effect also depends on the target length along the beam and HMS

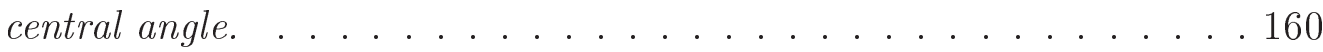

4.3 Simulated HMS pion decay distance for pions that decay and for which the resulting muon falls within the acceptance and passes all cuts. A large fraction of all simulated muons that pass all cuts come from the region after the HMS dipole and inside the spectrometer hut. . . . . . 163

4.4 Feynman diagrams contributing to first order, internal, soft Bremsstrahlung radiation. Figure from reference [55]. . . . . . . . . 169

4.5 Feynman space diagrams representing virtual photon corrections to one photon exchange cross section. Figure from reference [55]. . . . 170

4.6 Missing energy distribution for one of the ${ }^{1} H\left(e, e^{\prime} p\right)$ kinematics. Elastic data are used to distinguish resolution from radiative effects. In the elastic case the Monte Carlo simulation (solid line) describes the data (crosses) in the radiative tail quite well. The data/SIMC ratio changes by $1 \%$ over the entire missing energy acceptance. The discrepancy at low missing energy is due to resolution effects. . . . . . 172

4.7 Feyman diagrams of the dominant contributions of the two photon exchange effect. . . . . . . . . . . . . . 173

4.8 Ratio of data to Monte Carlo yields for elastic data taken for the four $F_{\pi}$-2 kinematic settings. The top panel shows the coincidence yields (proton absorption correction applied) and the bottom panel shows the electron yields. The error bars shown are statistical only. The dashed lines indicate the point to point errors between the data points and the solid lines indicate the overall systematic uncertainty. . . . . . . 178

5.1 Phase space in $W$ and $Q^{2}$ at both low and high $\epsilon$ points for the two dynamic settings. Note that $-t$ and $\theta^{*}$ change in value as well across the $W-Q^{2}$ acceptance. . . . . . . . . . . . . 182 
5.2 Representative plot of the experimental cross sections, $\frac{d^{2} \sigma}{d t d \phi}$ as a function of azimuthal angle $\phi$ at $Q^{2}=1.6(\mathrm{GeV} / \mathrm{c})^{2}$ for high and low $\epsilon$. The curves shown represent the fit of the measured values of the cross section to equation ??. In this fit all four parameters (the L,T,LT and TT response functions) are fitted simultaneously. . . . . . . . . 192

5.3 Comparison of Data (crossed) and SIMC (solid line) for the relevant pion electroproduction physics quantities, $W, Q^{2},-t, \phi$ and $\theta$. The Monte Carlo is weighted by the model input cross section. The distributions are normalized to each other by a global scale factor. . . . . 195

5.4 Ratio of data and simulated charge normalized yields across the $y_{\text {tar }}^{\prime}$ acceptance for elastic $e-p$ data at $P_{H M S}=3.83(\mathrm{GeV} / \mathrm{c})$ and $\theta_{H M S}=12^{\circ} .197$

6.1 Experimental unseparated cross sections at $Q^{2}=1.60(\mathrm{GeV} / \mathrm{c})^{2}$. The solid and dashed lines indicate the model prediction for hgigh and low

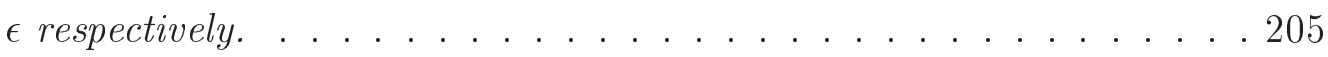

6.2 Experimental separated cross sections, $\sigma_{L}$ and $\sigma_{T}$ compared to $V G L$ Regge calculations with values of $\Lambda_{\pi}^{2}=0.512$ (0.491) $\mathrm{GeV}^{2}$ at values of $Q^{2}=1.60$ (2.45) $(\mathrm{GeV} / \mathrm{c})^{2}$. The error bars indicate statistical and random systematic uncertainty combined in quadrature. The error band denotes the correlated part of the systematic uncertainty by which all data points move collectively. The dashed line and dashed-dotted lines indicate VGL Regge calculations for $\sigma_{T}$ for values of $\Lambda_{\rho}^{2}$ of 0.6 and

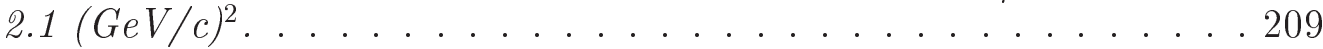

6.3 Experimental separated cross sections, $\sigma_{L T}$ and $\sigma_{T T}$ obtained from a 4 parameter fit to the experimental $\phi$ dependence. The error bars are statistical only. The solid and dashed lines represent the VGL Regge model results for $\sigma_{L T}$ and $\sigma_{T T}$ with $\Lambda_{\pi}^{2}=0.512(\mathrm{GeV} / \mathrm{c})^{2}$ and $\Lambda_{\pi}^{2}=0.491(\mathrm{GeV} / \mathrm{c})^{2} \ldots \ldots \ldots \ldots . \ldots \ldots$

$6.4 \chi^{2}$ distribution for a comparison of $\sigma_{L}$ from VGL/Regge calculations and $F_{\pi}-2$ experimental data. The data are fit with a parabola and the value of $F_{\pi}$ is extracted at the minimum of the $\chi^{2}$ distribution (solid line). The dashed line indicates the uncertainty in $\Lambda_{\pi}^{2}$ when $\chi^{2}$ varies by one standard deviation. . . . . . . . . . . . . . . . . . 212 
6.5 Pion form factor from $F_{\pi}$-2 compared to data from CERN, DESY and $J L a b F_{\pi}-1$. The error bars combine the statistical and experimental systematic uncertainties in quadrature. The curves are from a DysonSchwinger equation (dashed-dotted), QCD sum rules (dashed) and from a $p Q C D$ calculation (dotted). All models describe the data well up to values of $Q^{2}=1.60(\mathrm{GeV} / \mathrm{c})^{2}$. The $F_{\pi}$-2 data point at $Q^{2}=1.60$ $(\mathrm{GeV} / \mathrm{c})^{2}$ deviates by $\approx 1 \sigma$ from the Dyson Schwinger calculation by Maris and Tandy. . . . . . . . . . . . . . . . 214

6.6 Missing mass distribution for $\pi^{+}$production from deuterium. Triangles indicate experimental data and the solid line is the Monte Carlo assuming quasifree production. The disagreement between data and Monte Carlo may be attributed to not taking into account $N N$ final states interactions in the Monte Carlo. . . . . . . . . . . . . . . . 216

6.7 Longitudinal $\pi^{-} / \pi^{+}$ratios. The error bars on the data points are statistical and systematic. . . . . . . . . . . . . . . 219

6.8 Pion form factor from $F_{\pi}$-2 compared to $e-p$ scattering data from CERN, and precision pion electroproduction from DESY and JLab $F_{\pi}$-1. The solid curve is a monopole fit to the CERN data [4]. The monopole prediction describes the data well up to $Q^{2}=1.60(\mathrm{GeV} / \mathrm{c})^{2}$. The $F_{\pi}$-2 data point at $Q^{2}=2.45(\mathrm{GeV} / \mathrm{c})^{2}$ deviates by $1 \sigma$ from the monopole form. . . . . . . . . . . . . . 222

6.9 Pion electroproduction cross sections at 11 GeV kinematics. The longitudinal and transverse terms become comparable at $-t=0.6(\mathrm{GeV} / \mathrm{c})^{2} .225$

A.1 Charge normalized yield as a function of beam current from the hydrogen target a measured in the HMS. The data points are normalized so that the value of the line fit to the data is one at zero beam current. The error bars are statistical only. The dashed lines denote the uncertainty in the BCM calibration as a function of beam current. . . 232

A.2 Charge normalized yield as a function of beam current from the deuterium target a measured in the HMS. The data points are normalized as described in Figure ??. . . . . . . . . . . . . . . . . 233

A.3 Charge normalized yield as a function of beam current from the solid carbon target a measured in the HMS. The data points are normalized as described in Figure ??. . . . . . . . . . . . . . . . . . . . . . . . . 234

A.4 Charge normalized yield as a function of beam current from the solid carbon target a measured in the HMS. The data points are normalized as described in Figure ??. . . . . . . . . . . . . . . . . . . 235 
A.5 Charge normalized yield as a function of beam current from the solid carbon target a measured in the HMS. The data points are normalized as described in Figure ??. . . . . . . . . . . . . . . . . . . 236

B.1 Representative example of Aluminum thermal contraction for Al6061T6. The solid curve denotes a parameterization to data between 4 and $300 \mathrm{~K}$, while the dashed line is a calculation assuming a linear Thermal Expansion Coefficient. The data deviate significantly from the linear prediction at low temperature approaching a constant value. Note that this behavior is similar for all Aluminum alloys. . . . . . . 239 
Chapter 1

Introduction

\subsection{Overview}

A fundamental challenge in nuclear physics is the description of hadrons in terms of the constituents of the underlying theory of strong interactions (Quantum Chromodynamics, QCD). The relevant degrees of freedom in QCD are quarks and gluons, and a variety of experimental results have been sucessfully explained using this framework [1]. However, the observable degrees of freedom (hadrons) are different from the constituent degrees of freedom in the QCD Lagrangian (current quarks and gluons) making up the hadronic bound states. Moreover, the quark and gluon constituents cannot be isolated due to quark confinement at large distances. The transition from quark-gluon degrees of freedom to hadron degrees of freedom is therefore of significant interest in characterizing hadrons from first principles. Static properties like total charge and magnetic moments of hadrons are typically taken into account by constituent quark masses while contributions of dynamical properties like interactions of gluons and sea quarks to charge and current distributions of hadrons are not yet fully understood. In particular, the most fundamental part of the theory, the binding and confinement of quarks and gluons into hadrons, remains to be explained in detail.

Due the structural similarities between QCD and the theory of electromagnetic interactions, Quantum Electrodynamics (QED), and from the relatively straightforward calculations of QED observables from first principles, it is tempting to assume that the problems in hadron physics could use the corresponding tools of 
perturbation theory in QCD. However, this hypothesis is not correct, and so it is difficult to make predictions for hadronic obervables. To understand quark binding in hadrons, at least two length scales have to be considered. Asymptotic freedom at short distances indicates the relevance of perturbative physics at large momentum transfer and allows for calculations from first principles via perturbative methods. Exact calculations are not possible at low momenta, and since binding is a long distance effect, non-perturbative methods must play an important role as well. For a complete description of composite objects, a consistent analysis including both length scales is required. However, in the absence of a complete solution to QCD the predictive power of the theory is limited and we mostly rely on the extraction of related information from experimental data in the non-perturbative sector. These data can be used to constrain effective models describing the non-perturbative part of strong interactions at low momentum transfer and to look for the onset of perturbative behavior.

Hadronic form factors provide important information about hadronic structure. Whereas the coupling of structureless particles to a photon can be parametrized in terms of constant coefficients like charge and magnetic moments the internal structure of composite particles is determined by momentum dependent functions, the electromagnetic form factors, which describe the distribution of charge and current.

One of the simplest hadronic systems available for study in its ground state is the pion, whose valence structure is a bound state of two quarks. The electromagnetic structure of a spinless particle such as the pion is determined by a single form factor. The pion charge form factor, $F_{\pi}$, is well defined to lowest order in QCD 
in the limit of very large values of $Q^{2}$ [2], the square of the four-momentum of the photon:

$$
F_{\pi}\left(Q^{2}\right)=8 \pi \frac{\alpha_{s} f_{\pi}^{2}}{Q^{2}} \quad\left(Q^{2} \rightarrow \infty\right)
$$

It depends solely on the strong coupling constant $\alpha_{s}$ and its normalization is fixed by the pion decay constant $f_{\pi}$, which is determined from weak decay of the pion $\left(\pi \rightarrow \mu+\nu_{\mu}\right)$

In the perturbative QCD (pQCD) description, the high momentum (hard) components of the pion wave function are taken into account whereas the low momentum (soft) components cannot be suitably described. Due to the simple $q \bar{q}$ structure and minimal number of exchanged gluons in nuclear interactions the transition from soft (nonperturbative) to hard (perturbative) physics is expected to occur at significantly lower values of $Q^{2}$ for $F_{\pi}$ than the nucleon form factor [3] and is therefore more likely to be accessible experimentally.

The experimental determination of $F_{\pi}$ at large values of $Q^{2}$ is difficult due to the lack of a free pion target. In particular, determining the charge radius of the pion from elastic $\pi-e$ scattering experiments as described in $[4,5]$ is limited to relatively small values of $Q^{2}\left(\approx 0.28(\mathrm{GeV} / \mathrm{c})^{2}\right)$. The extension of the measurement of $F_{\pi}$ to larger values of $Q^{2}$ requires the use of pion electroproduction from a nucleon target (see Figure 1.12(a)) where a virtual photon couples to a pion inside the proton. The pion exchange process, which dominates the longitudinal forward electroproduction cross section, is due to the exchange of a longitudinally polarized virtual photon and determines the pion form factor. The pion electroproduction mechanism depends on 


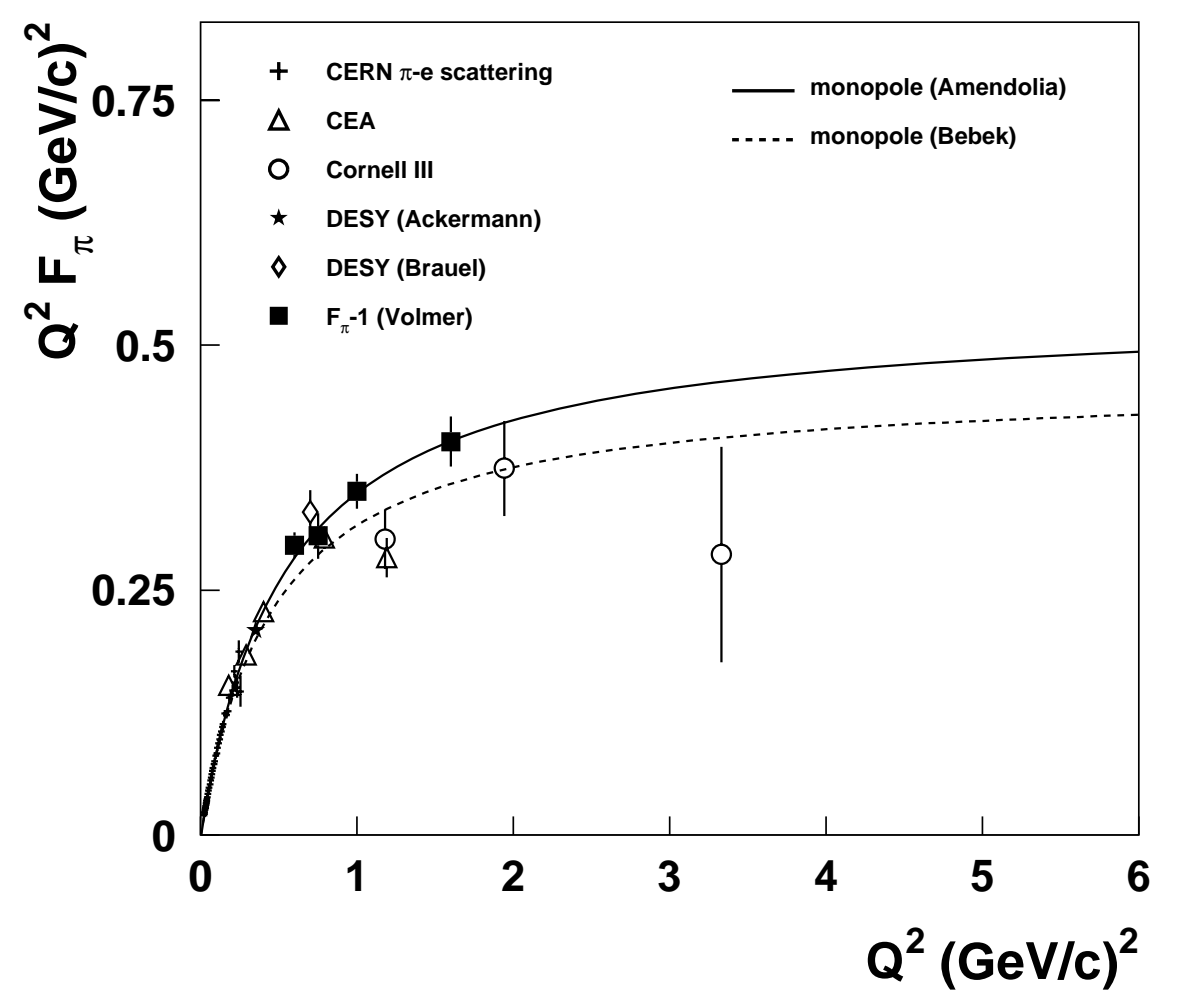

Figure 1.1: $F_{\pi}$ elastic $\pi$-e data from $C E R N$ and pion electroproduction data from CEA, Cornell III, DESY. The error bars are statistical only. Also shown are $F_{\pi}$ pion electroproduction $L / T$ data from JLab Hall $C\left(F_{\pi}-1\right)$. The solid curve is a monopole fit to the CERN data $\left(r_{\pi}=0.662 \mathrm{fm}\right.$, Ref. [4]). The dashed curve is a monopole fit to the Cornell III analysis result $\left(r_{\pi}=0.711 \mathrm{fm}\right.$, Ref. [6]).

$t$, the squared momentum transfer to the nucleon and $\mathrm{g}_{\pi N N}^{2}(\mathrm{t})$, the $\pi N N$ coupling constant. At least two theoretical issues arise in the extraction of the $F_{\pi}$ from electroproduction data. First, since the struck pion is off mass shell the extraction of $F_{\pi}$ from experimental data requires the extrapolation to the physical pion mass pole. This extrapolation requires knowledge of the $t$-dependence of the experimental cross section. Uncertainties in the knowledge of the $t$-dependence in the extrapolation will result in uncertainties in $F_{\pi}$ [9]. Secondly, other hard, nonresonant mechanisms may compete with the $t$-channel process at high $Q^{2}$ and the separation of mechanism may be difficult [10]. Both of these issues are part of the current experimental 
investigation and will be addressed in detail in subsequent chapters. It should be noted at this point that recent experimental results show relatively good agreement for values of $F_{\pi}$ determined from $\pi-e$ scattering and pion electroproduction. A summary of existing data is shown in Figure 1.1.

The separation of the longitudinal piece of the cross section, $\sigma_{L}$, from the total cross section is done via the Rosenbluth separation method which entails measurements at different photon polarizations, $\epsilon$, at fixed values of $Q^{2}$ and invariant mass of the photon-nucleon system, $W$. The pole dominance is tested using the ratio of longitudinal $\pi^{-}$and $\pi^{+}$cross sections on deuterium, which should be identical. The strong $t$-dependence from the $\pi$-N vertex and extrapolating to the minimum allowable value of $t$ allows then for the extraction of $F_{\pi}$. However, the $t$-dependence of $\sigma_{L}$ must be described via a theoretical model in which $F_{\pi}$ is a free parameter. Typical theoretical models used for this purpose are based on Regge theory or simple Born term models. While Regge theories describe pion electroproduction in terms of the exchange of $\rho$ - and $\pi$-like particles [11], Born term models are based on the lowest order Feynman pole diagrams with single particle exchange [12, 13].

Experimental $F_{\pi}$ data have previously been obtained at DESY [14, 15], CEA and Cornell $[6,7,8]$. Pion electroproduction data were obtained at DESY for a value of $Q^{2}$ of $0.7(\mathrm{GeV} / \mathrm{c})^{2}, W=2.19 \mathrm{GeV}$ and longitudinal and transverse cross sections were extracted using the Rosenbluth separation method. Most of the high $Q^{2}$ data have come from experiments at Cornell covering a range of values in $Q^{2}$ between 0.28 and $9.77(\mathrm{GeV} / \mathrm{c})^{2}$. In these experiments data were taken at only one beam energy and one value of $Q^{2}$, so the longitudinal cross section could not be extracted by a 
true Rosenbluth separation. The interpretation of the data, for which a Rosenbluth separation was attempted, is complicated by large systematic uncertainties. These uncertainties arise from the method used to separate the longitudinal and transverse pieces in the data. In order to isolate the longitudinal term, the transverse cross section was taken to be proportional to the total photoproduction cross section and subtracted from the measured total cross section. In the case of the lack of a consistent set of high and low $\epsilon$ data, the transverse piece of the cross section was estimated from an extrapolation of a set of Rosenbluth separated data. For the extraction of $F_{\pi}$ from the measured cross section, Born term models $[13,12]$ were used in both experiments. Therefore, though reliable pion electroproduction data exist for $Q^{2}<1 \mathrm{GeV}^{2}$, the high $Q^{2}$ data points provide no clear constraint in constraining theoretical models.

The Jefferson Lab $F_{\pi}$ program was designed to provide reliable L-T separated pion electroproduction data for values of $Q^{2}$ between 0.7 and $6(\mathrm{GeV} / \mathrm{c})^{2}$. The first measurement was performed in 1997 for values between $Q^{2} 0.6$ and $1.6(\mathrm{GeV} / \mathrm{c})^{2}$ at $\mathrm{W}=1.95 \mathrm{GeV}$. These data provide a determination of $F_{\pi}$ with a significant improvement in precision, over earlier experiments including the model dependence of the pion mass pole extraction in the experimental uncertainties [16]. The goal of the second phase of the measurement was to extend the dynamic coverage to $Q^{2}=2.5$ $(\mathrm{GeV} / \mathrm{c})^{2}$ and to improve the understanding of model dependence in the extraction of $F_{\pi}$ at higher values of $Q^{2}[17,18]$. The increase in dynamic range may rule out some effective nonperturbative calculations and may provide an indication about the higher $Q^{2}$ behaviour of $F_{\pi}$. To reach the kinematic region where perturbative 
QCD expectations may be approached, higher electron beam energies such as the ones proposed for the $12 \mathrm{GeV}$ upgrade to Jefferson Lab are required. To this end the third stage of the measurement of $F_{\pi}$ at Jefferson Lab is being planned for that time.

The following sections will illustrate in detail the description of the pion form factor at short and long distance scales and describe the experimental method used to extract the pion form factor at moderate values of $Q^{2}$. 


\subsection{The Pion Form Factor in the Asymptotic Limit}

The electromagnetic form factor of the pion can be expressed in terms of the matrix element

$$
\left(p_{1}+p_{2}\right) F_{\pi}\left(Q^{2}\right)=<\pi\left(p_{2}\right)\left|J_{\mu}(0)\right| \pi\left(p_{1}\right)>
$$

where $F_{\pi}$ is the electromagnetic pion form factor. $J_{\mu}=\sum_{f} e_{f} \bar{q}_{f} \gamma_{\mu} q_{f}$ is the electromagnetic current operator expressed in terms of the quark fields $q_{f}$ of flavor $f$ and electromagnetic charges $e_{f} \cdot J_{\mu}$ describes the coupling of photons to quarks. The quantities $p_{1}$ and $p_{2}$ denote the four-momenta of the incoming and outgoing pions respectively and $Q^{2}$ is the squared four momentum transfer to the photon.

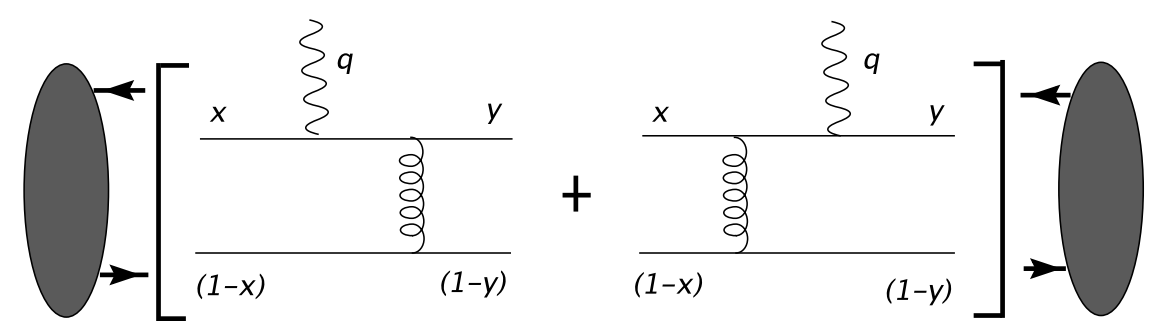

Figure 1.2: $F_{\pi}$ in hard scattering in the valence quark picture to lowest order. The photon couples to a quark with subsequent gluon exchange. The quantities $x$ and $y$ indicate the momentum fractions carried by individual valence quarks in the inital and final state respectively.

In the limit of very large momenta, $F_{\pi}$ can be calculated in perturbative QCD by isolating the short distance part of the interaction. The factorization theorem, which states that a physical amplitude can be expressed as a convolution of a hard (perturbative) scattering kernel and hadronic distribution amplitudes [19], provides the theoretical tool to separate short and long distance scales. In this framework the 
hadron is considered to be a definite partonic state and the hadronic wavefunction is thought of as a distribution of valence quarks, which includes all long distance dynamics. It should be noted that the momentum distribution of partons in each hadron are assumed to be entirely longitudinal. The behaviour of $F_{\pi}$ can then be expressed as a sum over wave functions involving only quark momentum fractions:

$$
F_{\pi}\left(Q^{2}\right)=\iint d x d y \phi_{\pi}\left(y, \mu^{2}\right) T\left(y, x, Q^{2}, \mu^{2}\right) \phi_{\pi}\left(x, \mu^{2}\right)
$$

where $\phi_{\pi}\left(x, \mu^{2}\right)$ is the valence state wave function describing a quark with fraction $x$ of the pion momentum and $T\left(x, y, Q^{2}, \mu^{2}\right)$ describes the hard scattering of partons. The integral is a perturbative expansion in the strong coupling constant, $\alpha_{s}\left(\mu^{2}\right)$, at a scale $\mu^{2}$. The hard scattering kernel can be expressed as the sum of gluon exchange diagrams contributing at lowest order as shown in Figure 1.2. Corrections to the hard scattering amplitude are supressed relative to hard scattering by additional inverse powers of $Q^{2}$ including soft, nonperturbative contributions [19, 20]. It follows that the one gluon exchange term dominates large values of $Q^{2}$. The hard scattering kernel has dimensions of inverse mass squared and scales as $1 / Q^{2}$. The explicit lowest-order form of $T$ is given by $[2,19]$.

$$
T=16 \pi C_{F} \alpha_{s}\left(\mu^{2}\right)\left(\frac{2}{3} \frac{1}{x y Q^{2}}+\frac{1}{3} \frac{1}{(1-x)(1-y) Q^{2}}\right)
$$

where $C_{F}=\left(N^{2}-1\right) / 2 N=4 / 3$ and $N$ denotes the number of colours in QCD. The product $x y Q$ is the virtuality of the exchanged gluon. The gluon virtuality 
determines the scale for the strong coupling constant $\alpha_{s}$ and the applicability of pQCD to the process. Full one-loop calculations have been performed for the pion form factor [21]. With a particular choice of scale $(Q=\mu)$, contributions to $T$ are limited to processes with transverse separation distances of $1 / Q$ and $T$ can be expanded in terms of the strong coupling, $\alpha_{s}$ alone.

The pion distribution amplitude can be calculated explicitly from the evolution with respect to the renormalization scale $\mu$ to all orders in $\alpha_{s}$ [21]. The most general solution is given in terms of an expansion in Gegenbauer polynomials, which are a class of orthogonal polynomials, as discussed in detail in reference [19]. Using this functional form, the pion's quark wave function can then be expressed in the limit of large $Q^{2}$ in the simple form [19]:

$$
\phi_{\pi}\left(x, \mu^{2}\right) \rightarrow \sqrt{3} f_{\pi} x(1-x)
$$

where the normalization $f_{\pi}=133 \mathrm{MeV}$ is known from weak decay of charged pions. It should be noted that the asymptotic wave function in equation 1.5 is model independent. The asymptotic form of $F_{\pi}$ to lowest order in pQCD follows from substitution of equation 1.5 into equation 1.3 and depends only on the QCD coupling constant, $\alpha_{s}$, with a normalization given by the constant $f_{\pi}$ determined from weak pion decay [2]:

$$
F_{\pi}\left(Q^{2}\right)=8 \pi \frac{\alpha_{s} f_{\pi}^{2}}{Q^{2}} \quad\left(Q^{2} \rightarrow \infty\right)
$$

where the high energy expression of the pion form factor is valid up to corrections 
in $\alpha_{s} \sim 1 / \ln (Q)[19,20]$.

The pQCD prediction for $F_{\pi}$ differs significantly from existing experimental results. Figure 1.3 shows existing data as a function of momentum transfer, $Q^{2}$. In particular, for $\alpha_{s} \sim 0.3$ at $Q^{2} \sim 1 \mathrm{GeV}^{2}$ the data exceed the QCD prediction by a factor of three. The apparent discrepancy between experimental data and the pQCD prediction was quickly attributed to the lack of including low momentum contributions to the pion wave function whose contribution become important at moderate and low values of $Q^{2}[3]$.

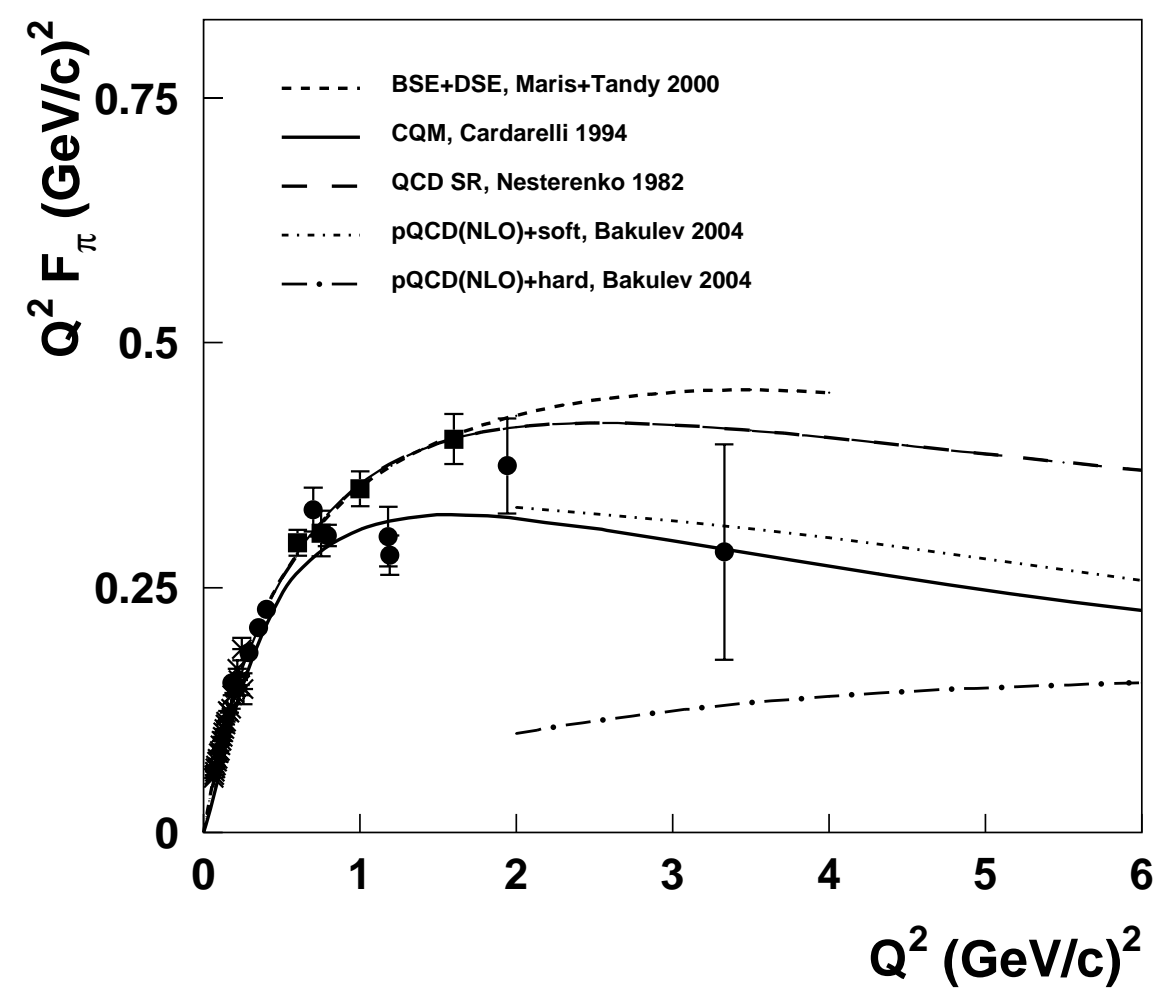

Figure 1.3: $F_{\pi}$ theoretical predictions from a constituent quark model (solid line, Ref. [22]), QCD sum rules (long dashed, Ref. [23]), Bethe-Salpeter/ DysonSchwinger equations (short-dashed, Ref. [24]) and next to leading order perturbative QCD with and without estimated soft contributions (dashed-dotted, Ref. [26]). The $F_{\pi}$ data are as in Figure 1.1.

Isgur and Llewellyn-Smith [3] and Bakulev and Radyushkin [27] pointed out 
that the application of the standard pQCD framework is complicated by the "endpoint problem" at moderate momentum transfers. At experimentally accessible energies the pion distribution amplitude contains substantial contribution from regions of $x$ or $(1-x)$ close to zero where the hard scattering expands in transverse space and the exchanged gluon carries a small momentum, thus violating the perturbative assumption [28]. For equation 1.3 to be applicable at experimentally accessible momentum transfer, the endpoint problem has to be addressed. This is typically done with the "Sudakov supression" mechanism, which effectively suppresses nonperturbative contributions to the interaction. The Sudakov corrections result from a resummation of higher order terms and damp contributions from the valence region in which the transverse momenta of the quarks are not large enough to prevent the exchange of soft gluons (gluonic radiative corrections). The transverse separation is accounted for by re-expressing $F_{\pi}$ in terms of a convolution of wave functions characterized by both fractional momenta and transverse separation. Sterman, et al. calculated $F_{\pi}$ using a Sudakov resummed transverse wavefunction to suppress the non-perturbative contribution at lower $Q^{2}[28,29]$. By suppressing configurations for which quarks are separated far enough to couple strongly to radiated gluons, the validity of perturbative calculations at low values of $Q^{2}$ was expected. However, Jakob and Kroll $[25,30]$ showed that the Sudakov corrections alone do not suppress the non-perturbative contributions entirely. They included an additional intrinsic transverse momentum dependence in the pion distribution amplitude re-expressing $\left(x y Q^{2}\right)^{-1}$ with $\left(x y Q^{2}+\left(k_{\perp}-l_{\perp}\right)^{2}\right)^{-1}$ where $k_{\perp}$ and $l_{\perp}$ are the transverse momenta of the intial and final state valence quarks respectively. The result of suppressing 
the transverse momentum contributions with additional strength was thought to extend the validity of perturbative calculations to a region as low as $1-4(\mathrm{GeV} / \mathrm{c})^{2}$ in $Q^{2}$.

A variety of improved perturbative calculations of $F_{\pi}$ exist. However, at lowest order, most of these calculations disagree with experimental data by more than a factor of 2 in the nonasymptotic regime. The discrepancy is generally attributed to soft contributions to the pion wave function at finite $Q^{2}[20,19]$. To estimate the weight of soft contributions, Braun, et al. [31] performed light cone sum rule calculations up to twist 6 and showed that hard components of higher twist partially cancel soft contributions even at moderate values of $Q^{2}$. The combination of soft and NLO perturbative contributions is on the order of $30 \%$ at $Q^{2} \sim 1(\mathrm{GeV})^{2}$.

\subsection{Pion Form Factor Dynamics}

The valence pQCD formalism allows for the calculation of $F_{\pi}$ at very high $Q^{2}$. However, to extend the calculation to non-asymptotic $Q^{2}$ it is necessary to develop methods that take into account processes that are suppressed by powers of $Q^{2}$ at high energies, and include a variety of soft processes. The complexity of soft, nonperturbative processes in QCD complicates the construction of simple physical descriptions connecting the low and high $Q^{2}$ regions. Whereas chiral perturbation theory and pQCD are effectively first principle techniques describing the strong interaction in the low and high energy domains separately, no comparably rigorous technique exists for the intermediate energy region. Constructing a method to describe strong interactions at intermediate energies in fundamental QCD remains one 
of the theoretical challenges in nuclear physics. With the requirement of minimal model-dependence a variety of calculations for $F_{\pi}$ can be grouped into at least three categories, which are described next.

\subsubsection{QCD Sum Rules}

QCD sum rules rely on quark hadron duality, which entails expressing the same object in terms of either quark or hadronic fields [27], and makes use of causality and the analyticity properties of Green's functions that are associated with the form factor. The relevant Green's function describing the quark content can be expressed in standard operator product expansion and the hadron content is given by the double dispersion relation:

$$
T\left(p_{1}^{2}, p_{2}^{2}, Q^{2}\right)=\frac{1}{\pi^{2}} \int d s_{1} \int d s_{2} \frac{\rho\left(s_{1}, s_{2}, Q^{2}\right)}{\left(s_{1}-p_{1}^{2}\right)\left(s_{2}-p_{2}^{2}\right)} .
$$

where $p_{1}, p_{2}$ and $q$ are the four momenta of the inital and final pion and the photon respectively. The spectral density function $\rho$ contains a pion pole giving $F_{\pi}$, and is defined as $\rho_{\pi \pi}\left(s_{1}, s_{2}, Q^{2}\right)=2 \pi f_{\pi}^{2} F_{\pi}$, where $f_{\pi}^{2}$ in this expression denotes the pion decay constant. The spectral function describes the continuum above the three pion threshold in terms of $q \bar{q}$ condensates with invariant masses $s_{1}$ and $s_{2}$. Using standard Green's function techniques the values for $s_{0}$ and $M$, the effective threshold for higher states production and adjustable mass in the duality interval, respectively, can be determined. The soft and hard contributions expressed as a relation between $F_{\pi}\left(Q^{2}\right)$ and the lowest order perturbative contribution to the spectral density, $\rho$, 
can then be expressed as follows:

$$
\begin{aligned}
& F_{\pi}^{s o f t}\left(Q^{2}\right)=1-\frac{1+6 \frac{s_{0}}{Q^{2}}}{\left(1+4 \frac{s_{0}}{Q^{2}}\right)^{3 / 2}} . \\
& F_{\pi}^{\text {hard }}\left(Q^{2}\right)=\frac{\alpha_{s}}{\pi} \frac{1}{\left(1+\frac{Q^{2}}{2 s_{0}}\right)^{3 / 2}} .
\end{aligned}
$$

Expanded in inverse powers of $Q^{2}$, expression 1.8 shows nonleading behaviour compared to the perturbative prediction. The hard, perturbative contribution in equation 1.9 is estimated by interpolating between asymptotic values of $Q^{2}$. Bakulev, et al. combined equations 1.8 and 1.9 for their prediction of $F_{\pi}$ and find relatively good agreement with experimental data [27] (see Figure 1.3). This result confirms the hypothesis [3] that soft contributions play a significant role in $F_{\pi}$ and may dominate over perturbative contributions at moderate energies.

\subsubsection{Lattice Calculations}

Lattice QCD provides a relatively rigorous technique for describing strong interaction in the intermediate energy region. Numerical simulations of lattice QCD provide direct accessibility to the QCD equations of motion and results for the dressed quark two-point functions ("propagators") are in semi-quantitative agreement with Dyson-Schwinger equation calculations (a detailed discussion can be found in references $[33,34])$. Though based on first principles at least three approximations to the Lagrangian are necessary and contribute to the overall uncertainty in any lattice result. The most obvious uncertainty is associated with discretization 
errors due to lattice spacing. Additional uncertainties arise from the extrapolation of the lattice results to the physical pion mass and the neglecting of disconnected quark loops in the "quenched approximation" [36, 35]. Whereas discretization errors can be addressed using improved lattice actions that suppress lattice spacing errors, limitations in available computational power result in a standard lattice pion mass significantly larger than the physical pion mass [37]. The quenched approximation introduces an a priori not easily quantifiable systematic error. Unquenched (full QCD) lattice calculations were recently performed by Bonnet et al [37].

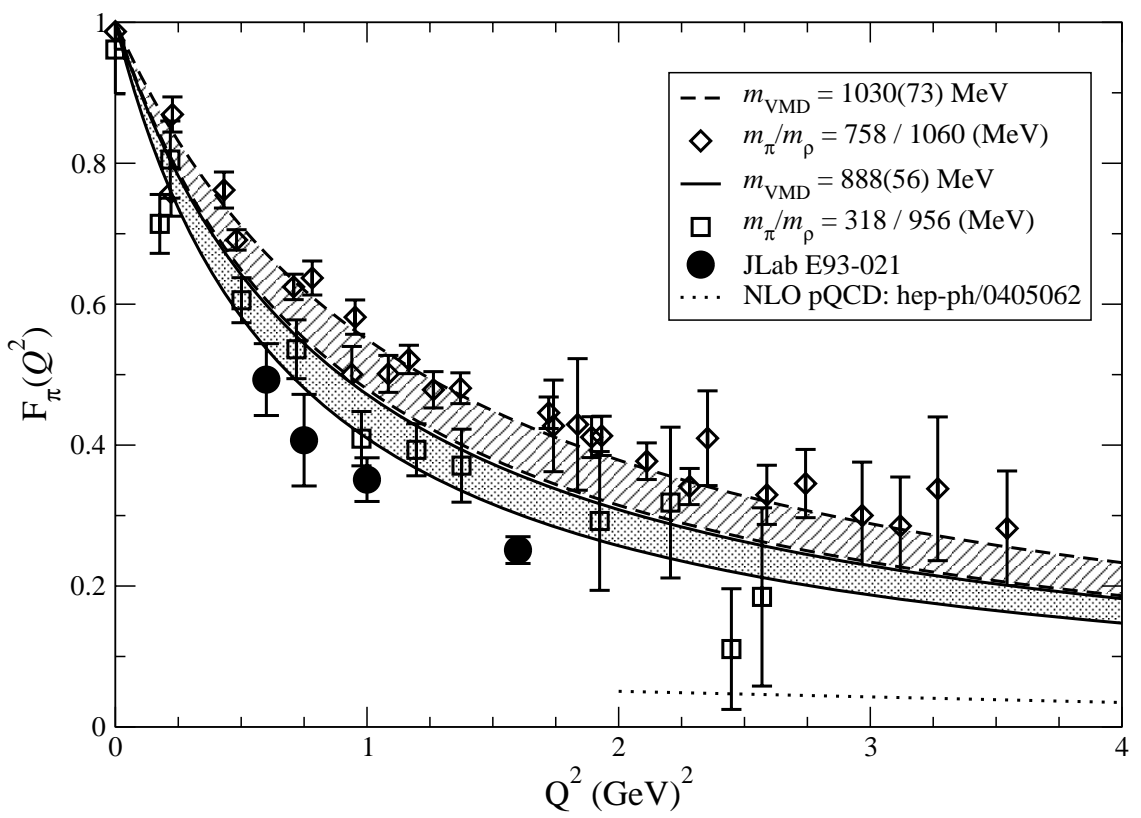

Figure 1.4: Lattice calculation of the pion form factor by Bonnet et al. [37]. The calculation uses a chiral-symmetry preserving domain wall action and lattice pion masses of $318 \mathrm{MeV}$ and $758 \mathrm{MeV}$. A domain wall on the lattice can be viewed as a plane separating degenerate solutions to the Dirac equation. The shaded regions indicate the uncertainty for a vector meson dominace fit. Note that no chiral extrapolation has been done in the lattice results shown in the figure. The datapoints shown are from $F_{\pi}-1$. 
Although the most recent lattice calculations have clearly improved over earlier work limited to values of $Q^{2}<1(\mathrm{GeV} / \mathrm{c})^{2}$ [38], the use of lattice simulations for a quantitative interpretation of experimental data is still in the early stages. One major effort in this direction was accomplished by Bonnet et al. [37], who provided the first unquenched lattice calculation for $F_{\pi}$. The result has strong $m_{\pi}$ dependence as shown in Figure 1.4. The lattice calculation with a pion mass of 318 $\mathrm{MeV}$ lies consistently below the calculation with a pion mass of $758 \mathrm{MeV}$. Though both calculations overpredict the experimental data, the lattice result suggests that an unquenched lattice calculation near the physical mass pole may result in good agreement with the data.

\subsubsection{Models}

Unlike numerical simulations of QCD on a finite spacegrid, continuum methods in hadronic physics are based on modelling. Constituent quark models describe the baryon spectrum and decay with a small number of parameters. However, this type of model does not provide a good description of the entire set of phenomena associated with the lightest mesons. Other models successful in describing many aspects of hadron physics are effective field theories, light-front calculations and approaches based on the Dyson-Schwinger equations.

i. Quark Hadron Duality

Quark hadron duality describes the transition from perturbative to non-perturbative regions in QCD in terms of the relation between confinement and asymptotic free- 
dom. The perturbative treatment of hard exclusive processes assumes a partonic description of the participating hadrons and is therefore closely related to the parton model of inclusive processes. Under the assumption of locality, hadronic structure contained in exclusive form factors can be related via quark hadron duality to inclusive structure functions. Duality in the pion can be understood as the relation between its effective hadronic description as the Goldstone boson of chiral symmetry breaking and its microscopic description as a quark-antiquark system. Analogous to calculations on the nucleon, the relation between the pion form factor and pion structure function, $F_{2}^{\pi}=\nu W_{2}^{\pi}$, can be expressed in terms of a dispersion integral. The upper limit in equation 1.10 denotes the elastic contribution to the inclusive structure function [39],

$$
F_{\pi}^{2}\left(Q^{2}\right)=\int F_{2}^{\pi}\left(\omega, Q^{2}\right) \omega d \omega
$$

In principal, quark hadron duality allows for the prediction of $F_{\pi}$ or $F_{2}^{\pi}$ given experimental data on the pion structure function or the pion form factor respectively. While a relatively large set of $F_{\pi}$ data is available from DESY, Cornell and JLab, experimental data on the pion structure function are sparse. In the valence region $(0.2<x<0.99)$, the quark distribution has been studied at Fermilab using the Drell-Yan mechanism [40]. Figure 1.5 shows the result of a local duality calculation of $F_{\pi}$ by Melnitchouk using the leading order analysis of the pionic Drell-Yan data on the pion structure function [41]. Note that the shape of the curve depends significantly on the $x$ dependence of the pion structure function. Melnitchouk's result (solid line in Figure 1.5) is in remarkably good agreement with the prediction from 
the Drell-Yan-West relation [42], which predicts $F_{2}^{\pi} \sim(1-x)$ under the assumption of the asymptotic form of $F_{\pi}$ at high energies. While leading order analysis of the experimental data from Fermilab are in relatively good agreement with this prediction $[43,44]$, a recent next to leading order analysis predicts significant deviation from linec

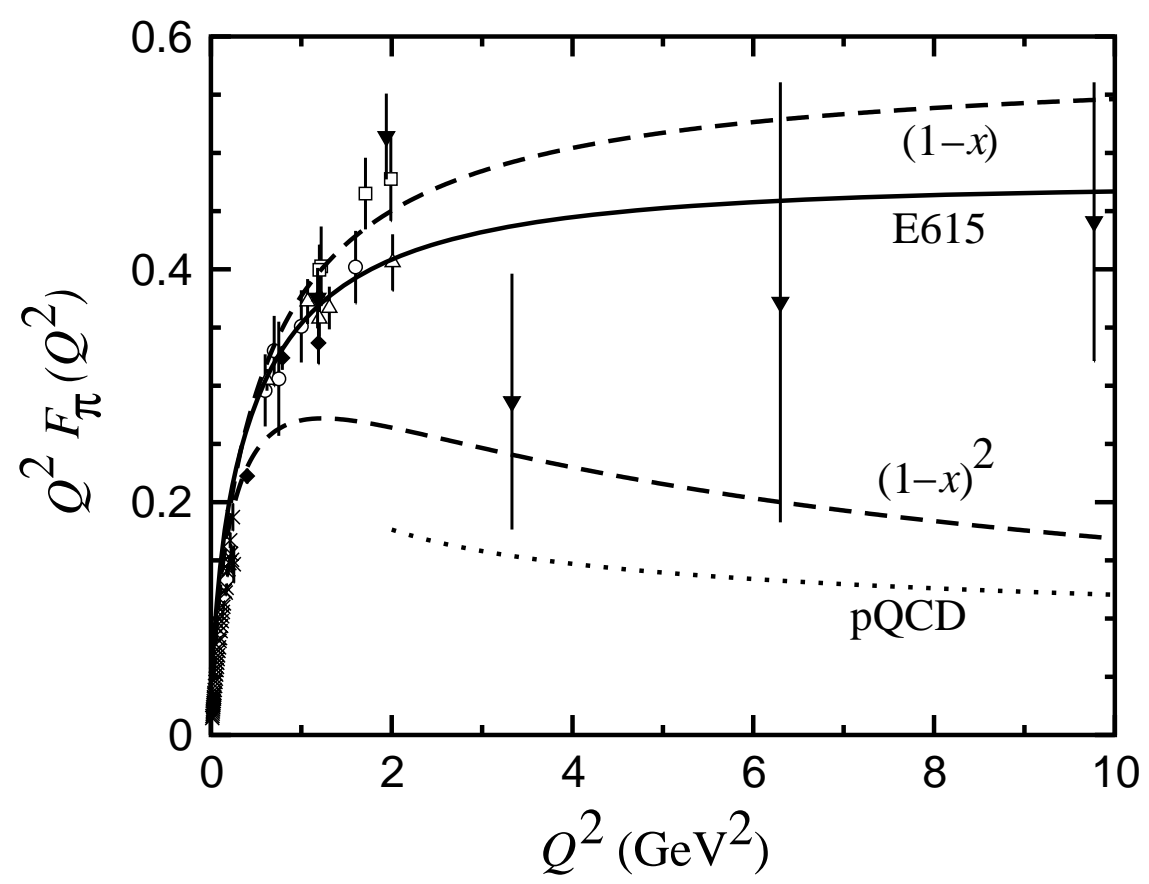

Figure 1.5: Local Duality prediction of the pion form factor using Fermilab E615 Drell-Yan data on the pion structure function. The leading order analysis of the experimental data was used in the calculation. The two dashed lines denote linear and quadratic $x$-dependeces. The dotted line is the asymptotic leading order $p Q C D$ prediction. The figure is taken from reference [41].

ii. Dyson-Schwinger Approach

The Bethe-Salpeter equation (BSE) provides a fully relativistic description of the two-body bound state problem analogous to the nonrelativistic Schroedinger equation. The static properties of the bound state are expressed in terms of the 
Bethe-Salpeter amplitude and the dynamics are summarized in a $q \bar{q}$ scattering kernel (Figure 1.6).

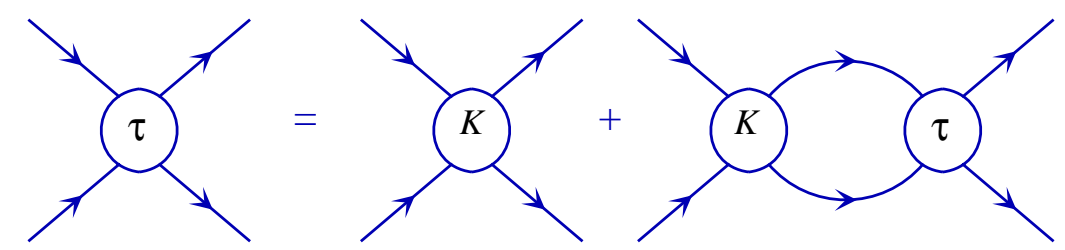

Figure 1.6: Diagram of the Bethe-Salpeter equation. Figure from reference [46].

Modeling QCD in the non-perturbative regime requires a proper description of medium effects resulting from the interaction between the constituents. This is addressed in light front Bethe-Salpeter models by separating the hard and soft components in the BSE. To obtain the essential non-perturbative features such as confinement and vacuum condensates, Jacob and Kisslinger [47] use a linear confining potential and a perturbative piece derived from one gluon exchange or dynamic chiral symmetry breaking. They predict an asymptotic value for $F_{\pi}$ at a value of $Q^{2} \sim 15(\mathrm{GeV} / \mathrm{c})^{2}$

In an alternative approach, Maris and Tandy obtain the Bethe-Salpeter amplitudes and the quark-photon vertex as the solution of the corresponding BSE in "ladder truncation", which uses as an input the dressed quark propagators derived from the "rainbow truncation" of the Dyson-Schwinger equations (DSE) [24]. This approach is in principal like the Hartree-Fock approximation in many-body physics.

The rainbow ladder describes the short range part of the interaction kernel fixed by perturbative QCD assuming one gluon exchange with one loop renormalization. The long range part of the interaction is fixed by the quark antiquark 
condensate where the gluon mass scale is set at $200 \mathrm{MeV}$. Conservation of the relevant currents in the truncation, allows for the pion to be generated as both, a bound state of massive constituents and as the Goldstone boson of chiral symmetry breaking. In addition, the model is Poincare invariant and consistent with quark gluon confinement. The model parameters are constrained by the requirement that the pion mass and the pion decay constant reproduce the experimental values and $F_{\pi}$ and the pion charge radius are obtained with no further adjustment of the parameters. The produced pion radius agrees with experimental data to $2 \%$ [48]. A detailed discussion of the DSE formulation can be found in reference [49].

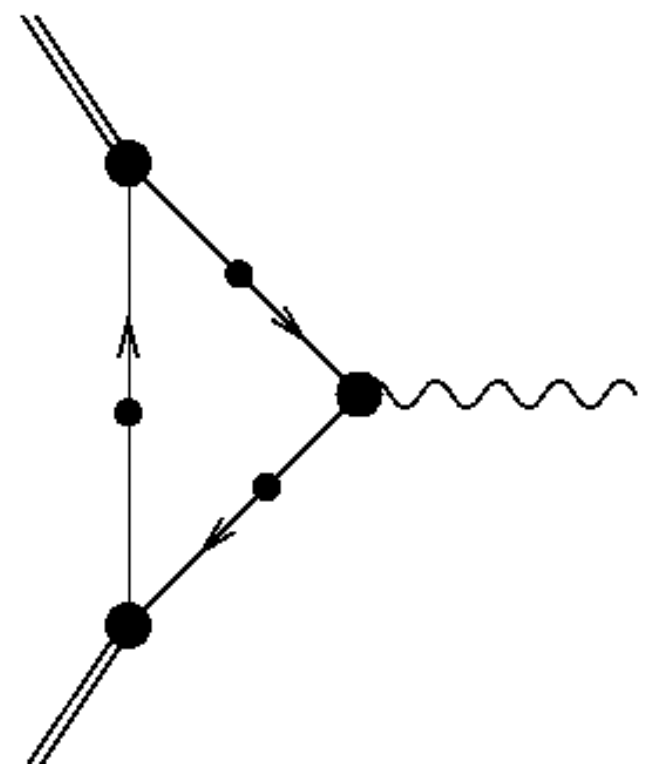

Figure 1.7: Impulse approximation for the pion form factor. The model amplitude for the mesons are the solutions of the homogeneuos Bethe-Salpeter equation, which has two inputs: 1) Quark propagator and 2) the scattering kernel. Integrating over one of the variables in the path integral containing all orders one arrives at a hierachy of coupled n-point functions. The calculation is usually performed in a Euclidean metric in momentum-space, which is the Fourier transform of the lattice approach. 
iii. Constituent Quark Models

Constituent quark models are effective theories constructing the pion from constituent quarks and describing the pion wavefunction in terms of solutions to a potential obtained from a $q \bar{q}$ interaction model. In order to extend the validity of the model to the non-perturbative regime the constituent quark model has been made fully relativistic [50, 22] allowing for a consistent, relativistic description of quark spins. A typically used framework is based on the Fock state decomposition of hadronic states, which arises in the "light-cone quantization". A light cone wave function is a localized stationary solution of the light cone Schroedinger equation describing the evolution of a particle state on the light cone time, $\tau=x^{0}+x^{3}$ in a particular light cone gauge. The square of the light cone wave function is the probability to find a constituent with specific momentum, $x P$ ( $x$ denotes the light cone momentum fraction), in a pion of momentum $P$. A diagram of a pair of valence quarks separated on the lightcone is shown in Figure 1.8. In the light cone frame the

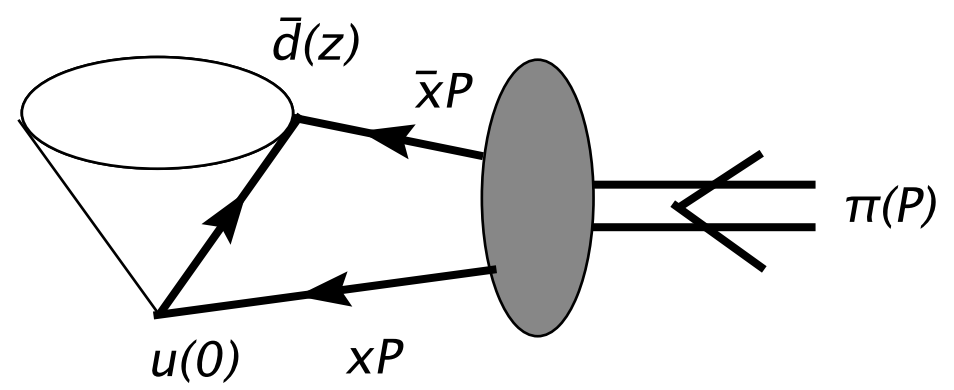

Figure 1.8: Diagram of transition of the physical pion to a pair of valence quarks separated on the lightcone. This process is described by the pion distribution amplitude.

wave function is factorized into a linear confining term and a one-gluon perturbative 
term $\psi\left(x, p_{T}\right) \sim X(x) P\left(x, p_{T}\right)$. The scale $p_{T}$ produces an effective cutoff in the UV region for which the one-gluon exchange term is not included in the form factor. The quark separation determines the significance of the soft contributions over the range of measured $Q^{2}[3]$. At least two variations of the model exist. Hwang, et al. [51] use a wave function determined from experimental data on the charged pion decay constant, the neutral pion two-photon decay width and the charged pion radius to predict the charge and transition form factors of the pion. Alternatively, existing $F_{\pi}$ data can be used to constrain the type of interaction model used for the constituent quarks in the pion and the range of the constituent quark masses [52]. In the framework of the relativistic Hamiltonian the dominant dependence is due to the constituent quark mass rather than the type of quark interaction model. In yet another approach Sengbusch and Polyzou [53] consider point-like constituent quarks under different confining potentials. The authors find that there exists much freedom to change representation and still fit the meson masses and the pion form factor (see Figure 1.9). This freedom may be used to simplify the treatment of dynamics in the model. Experimental data at higher $Q^{2}$ could provide greater selectivity for the pion wavefunction and quark mass value.

While the theoretical description of $F_{\pi}$ at low and high energies is well known, its calculation in the transition to the asymptotic regime remains complicated. Several effective models are available. However, by definition none of them can provide a prediction based entirely on QCD methods. In addition, model predictions begin to diverge at moderate values of $Q^{2}$. This is significant, because any effective theory that is correct, even if it requires input from other data, would give the correct be- 


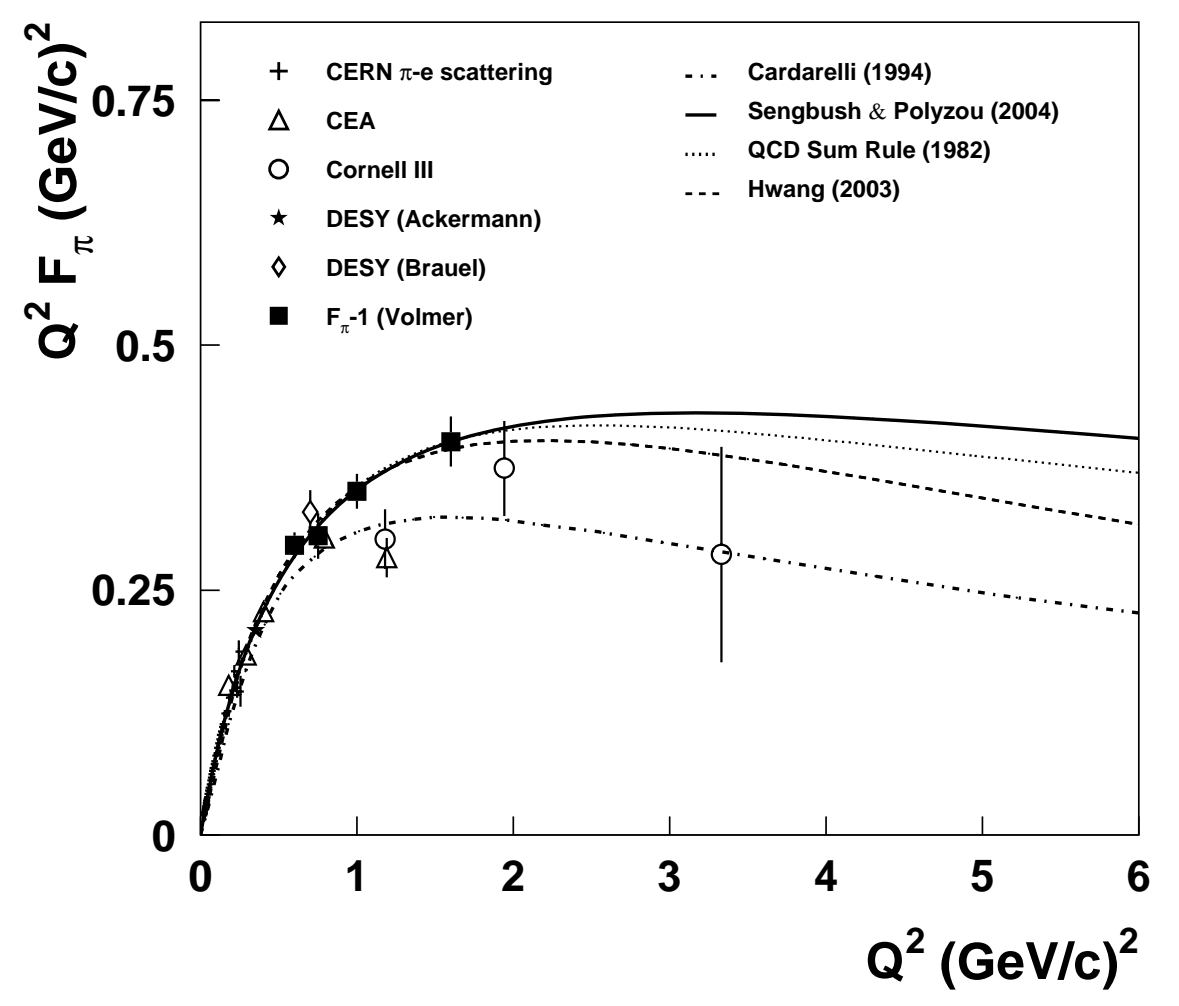

Figure 1.9: Consituent quark model predictions for the pion form factor compared to QCD sum rule and dispersion relation calculations. Shown are a constituent quark model calculation by Cardarelli (dashed-dotted, [22]), a more recent constituent quark analysis with different confining potentials (solid, [53]), QCD sum rules (dotted, [23]), and a dispersion relation calculation by Geshkenbein (dashed, [54]). The dispersion relation approach relates timelike and spacelike regions of the pion form factor in the complex plane and is generally rigorous. However, the approach is not well constrained in the timelike region.

havior at large values of $Q^{2}$. However, the free parameters in many effective models are not well constrained and experimental data are needed to constrain theoretical calculations in the intermediate dynamic range. 


\subsection{Pion Electroproduction}

\subsubsection{Kinematics Definition}

To study the $Q^{2}$ dependence of $F_{\pi}$, exclusive $\pi^{+}$production from the proton is used,

$$
e+p \rightarrow e^{\prime}+\pi^{+}+n
$$

where $p$ and $n$ denote the initial and final state of the nucleon. The kinematics

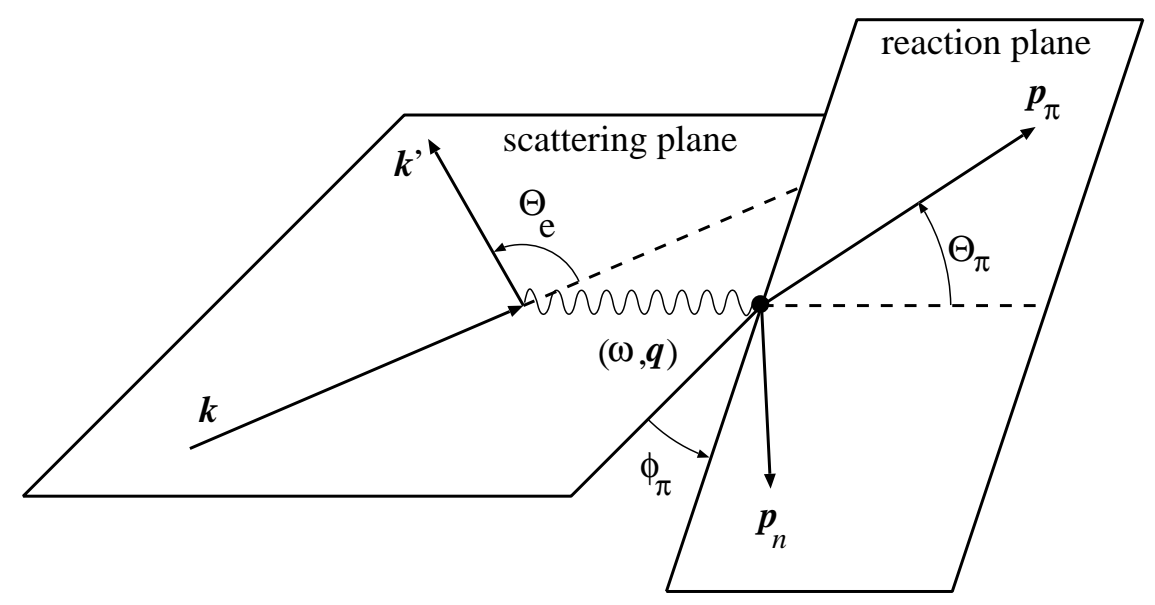

Figure 1.10: Schematic representation of the pion electroproduction process. The three-vectors of the initial and final electron define the scattering plane. The momentum transferred to the photon-nucleon system is defined in terms of the fourmomentum transfer, $Q^{2}$. The reaction plane is defined by the three-momentum vectors of the pion and the recoiling nucleon. The pion is emitted at an angle $\theta_{\pi}$ relative to the virtual photon direction and the angle between the scattering and reaction plane is $\phi_{\pi}$. Figure from reference [56]

of the $\mathrm{p}\left(e, e^{\prime} \pi^{+}\right) \mathrm{n}$ reaction using the standard notation for total energies and threemomenta is depicted in Figure 1.10. The incident electron interacts with the nucleon via virtual photon exchange. The scattering plane is defined by the three momentum vectors of the incoming and outgoing electrons denoted by $\mathbf{k}$ and $\mathbf{k}^{\prime}$ respectively. The electron scattering angle is denoted by $\theta_{e}$. The 4-momentum transferred by the 
electron is defined in terms of the 4-momentum $q=(\omega, \mathbf{q})=\left(k-k^{\prime}\right)$ of the virtual photon exchanged with the nucleon, where $\omega=E_{i}-E_{f}$. By definition, the square of the virtual photon 4-momentum vector $q^{2}=q_{\mu} q^{\mu}=\omega^{2}-|\mathbf{q}|^{2}$ is always negative in electron-nucleon scattering and henceforth the notation $Q^{2}=-q^{2}>0$ will be used for the space-like electron scattering process. The reaction plane is defined by the three-momentum vectors of the pion and the recoiling nucleon respectively. The pion is emitted at an angle $\theta_{\pi}$ relative to the virtual photon direction and the angle between the scattering and the reaction plane is denoted by $\phi_{\pi}$. Detecting the pion along $q$ with $\theta_{\pi}=0$ corresponds to "parallel kinematics".

The energy and three momentum of the recoiling nucleon system can be calculated by applying momentum conservation at the reaction vertex. If both the scattered electron and pion are detected and the incident electron kinematics are known, the corresponding missing energy $\left(E_{m}\right)$ and missing momentum $\left(P_{m}\right)$ are well determined,

$$
\begin{array}{r}
\mathbf{p}_{\mathbf{m}}=\mathbf{q}-\mathbf{p}_{\pi} \\
E_{m}=E_{e}-E_{e^{\prime}}-E_{\pi}
\end{array}
$$

The missing mass of the recoil system can then be expressed as $M=\sqrt{E_{m}^{2}-\mathbf{p}_{\mathbf{m}}^{\mathbf{2}}}$. In the case of the ${ }^{1} H\left(e, e^{\prime} \pi^{+}\right) n$ the missing mass is given by the neutron mass $M=m_{n}$. This assumption is not accurate in the case of pion electroproduction from deuterium because the extra degree of freedom introduced by the relative momentum between the recoiling nucleons does not allow for a discrete value of the 
missing mass.

Pion electroproduction is typically described in terms of the incident electron kinematics and the response functions which carry the hadronic information. The response functions are completely determined by the Lorentz invariants $Q^{2}, W$ and $t$, where $Q^{2}$ denotes the invariant mass of the exchanged virtual photon, $W$ is the square root of the total energy in the photon-nucleon center of mass frame and $t$ is the four-momentum squared of the momentum transferred to the nucleon. The invariant mass of the photon-nucleon system $W=\sqrt{s}$ can be expressed,

$$
W=\sqrt{M^{2}+2 M \omega-Q^{2}}
$$

where $M$ is the target mass (in this case the proton mass). The four-momentum transfer to the pion, $t=\left(q-p_{\pi}\right)^{2}$, in pion electroproduction can be written:

$$
t=\left(E_{\pi}-\nu\right)^{2}-\left|p_{\pi}\right|^{2}-|q|^{2}+2\left|\mathbf{p}_{\pi}\right||\mathbf{q}| \cos \theta_{\pi}
$$

which is always negative for the reaction considered, and henceforth the notation $-t$ will be used. The minimum value of $-t$, denoted by $-t_{\min }$, is attained at $\theta_{\pi}=0$, at the maximum value of the cosine. At fixed values of $Q^{2},-t_{\min }$ decreases with increasing values of invariant mass, $W$. 


\subsubsection{Cross Section Definition}

In the plane wave Born approximation, the incident and scattered electrons are described by Dirac plane waves and the interaction with the target is mediated by a single virtual photon (one photon exchange approximation). The six-fold $\left(e, e^{\prime}, \pi\right)$ differential cross section simplifies for discrete final states and can be expressed as the product of the virtual photon production cross section, $\frac{d \sigma}{d \Omega_{\pi}^{*}}$ and a virtual photon flux factor, $\Gamma$. In terms of the scattered electron energy $E_{e}^{\prime}$, electron solid angle, $d \Omega_{e}=d \sin \theta_{e} d \phi_{e}$ and pion solid angle in the center of mass, $d \Omega_{\pi}^{*}=d \sin \theta_{\pi}^{*} d \phi_{\pi}$ the pion electroproduction cross section and flux factor can be written in terms of:

$$
\frac{d^{5} \sigma}{d \Omega_{e} d E_{e}^{\prime} d \Omega_{\pi}}=J\left(t, \phi \rightarrow \Omega_{\pi}\right) \Gamma_{v} \frac{d^{2} \sigma}{d t d \phi_{\pi}}
$$

where $J\left(t, \phi \rightarrow \Omega_{\pi}\right)$ is the Jacobian transforming the hadronic part of the differential virtual photon cross section, and

$$
\Gamma_{v}=\frac{\alpha}{2 \pi^{2}} \frac{E_{e}^{\prime}}{E_{e}} \frac{1}{Q^{2}} \frac{1}{1-\epsilon} K
$$

is the virtual photon flux. $\alpha$ is the fine structure constant, and $\epsilon$ is the longitudinal polarization of the virtual photon,

$$
\epsilon=\frac{1}{1+2 \frac{\left|q^{2}\right|^{2}}{Q^{2}} \tan ^{2}\left(\theta_{e} / 2\right)}
$$


$K$ is the equivalent real photon energy required to excite a target of mass $\mathrm{M}$ to an excited state of mass W. In the Hand convention [57] $K$ is written:

$$
K=\frac{W^{2}-M^{2}}{2 M}
$$

The four unpolarized response functions, which constitute the fundamental contributions to the virtual photon cross section contain all information that can be extracted from the hadronic system. In particular, the four response functions correspond to the polarization states of the virtual photon, the longitudinal (L), transverse (T) and two interference terms (LT and TT), and can be directly related to the components of the hadronic current. In terms of the four response functions the cross section can be written as,

$$
2 \pi \frac{d^{2} \sigma}{d t d \phi_{\pi}}=\frac{d \sigma_{T}}{d t}+\epsilon \frac{d \sigma_{L}}{d t}+\sqrt{2 \epsilon(1+\epsilon)} \frac{d \sigma_{L T}}{d t} \cos \phi_{\pi}+\epsilon \frac{d \sigma_{T T}}{d t} \cos 2 \phi_{\pi} .
$$

Note that the four response funtions $\frac{d \sigma_{i}}{d t}$ depend only on $Q^{2}, \mathrm{~W}$ and $\mathrm{t}$.

The separation of the four response functions entails varying the values of $\epsilon$ and $\phi_{\pi}$ keeping the independent variables constant. The two interference terms can be eliminated either by taking data along the direction of the virtual photon (parallel kinematics), or by measuring these terms over the full angular $\phi$ range and integrating over the acceptance. In the former case, the virtual cross section becomes

$$
\frac{d \sigma}{d t}=\frac{d \sigma_{T}}{d t}+\epsilon \frac{d \sigma_{L}}{d t}
$$


The longitudinal and transverse contributions to the cross section can be separated via the Rosenbluth $(\mathrm{L} / \mathrm{T})$ separation method. This technique requires the measurement of the virtual photon cross section at two values of $\epsilon$ keeping $W, Q^{2}$ and $t$ fixed and fitting a straight line to the known $\epsilon$ dependence. The longitudinal cross section is given by the slope and the transverse cross section by the y-intercept $(\epsilon=0$ intercept). The photon polarization $\epsilon$ can be varied by changing the electron energy and scattering angle. In order to determine the interference terms the azimuthal angle between the scattering and production planes, $\phi_{\pi}$, is varied. For the case of $\sigma_{L T}$ this is achieved by measuring the pion to the left and right of the $q$-vector, while an additional out of (scattering) plane measurement is required for $\sigma_{T T}$.

\subsubsection{Pion Electroproduction Models}

The struck pion in the electroproduction mechanism is off mass shell and the extraction of the pion form factor from experimental data requires the extrapolation to the physical pion mass pole. This extrapolation requires knowledge of the $t$ dependence of the experimental cross section. Uncertainties in the knowledge of the $t$-dependence in the extrapolation will result in uncertainties in $F_{\pi}$ [9]. Secondly, other hard, nonresonant mechanisms may compete with the $t$-channel process at high $Q^{2}$ and the isolation of that mechanism may be difficult [10].

In the first order Born description, pion electroproduction processes are described in terms of the contributing covariant Born term diagrams (depicted in Figure 1.12). The pole process (a) corresponds to the knockout of a virtual pion by a virtual photon. Diagrams (b) and (c) show the nucleon pole and crossed nucleon 


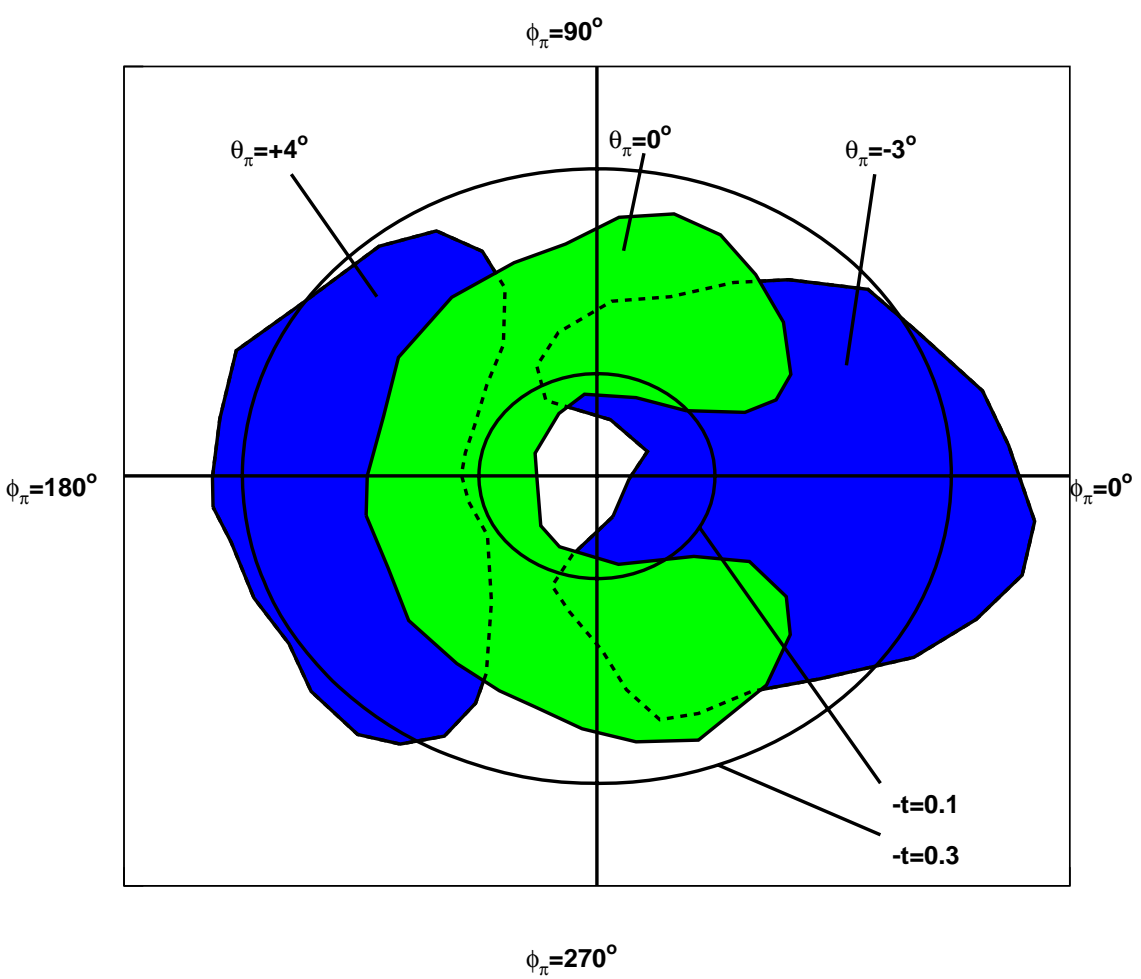

Figure 1.11: Polar plot of the $-t$ phase space. The radial component is $-t$ shown as two circles for values of $-t=0.1$ and $0.3(\mathrm{GeV} / \mathrm{c})^{2}$. The angular component is $\phi_{\pi}$ which progresses counter-clockwise with $\phi_{\pi}=0$ to the right. The full $\phi$ coverage is obtained for the high $\epsilon$ setting at three $H M S$ central angles $(0, \pm 3)$

pole and diagram (d) corresponds to the contact term, which is included to preserve gauge invariance when pseudovector coupling to the virtual photon is used. For the study of the electromagnetic structure of the pion, the diagram of interest is the pion pole. However, there is a nonzero probability for the other diagrams to contribute. In particular, the presence of off mass shell particle states or higher order processes requires a more complex description of the general structure of the electromagnetic vertex of hadrons and the associated form factors depend on more variables than they do in the free case. In particular, $\sigma_{T}$ can couple strongly to higher order processes such as nuclear resonances (e.g. $\Delta(1232)$ ), multiple pion exchange or exhange 
of heavy mesons $(\rho, \omega)$. The simplest Born term model assumes that other processes are suppressed in forward kinematics and that the longitudinal cross section is given by the Born exchange amplitude for values of $W$ above the resonance region.

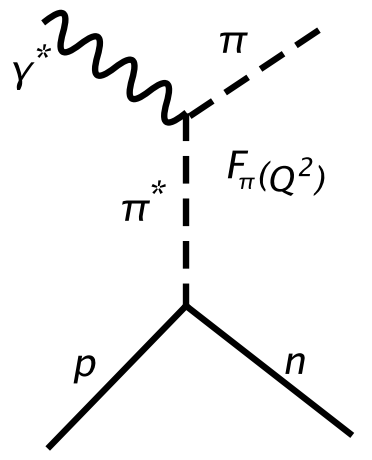

(a)

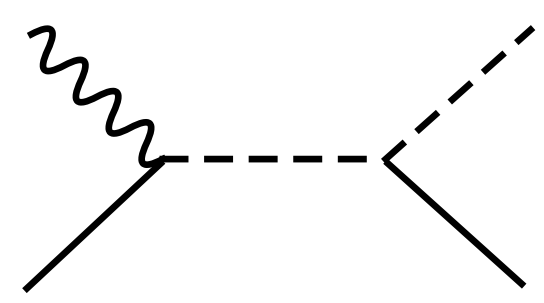

(b)

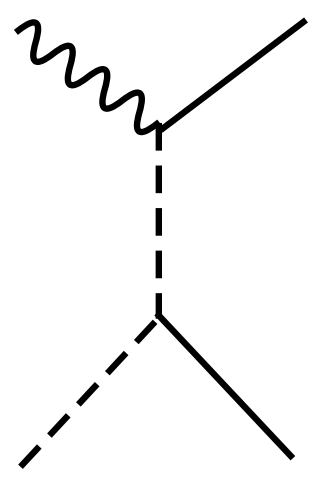

(c)

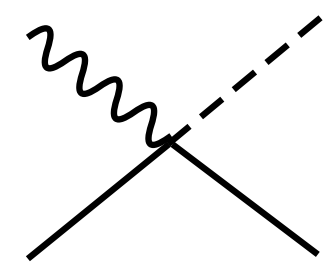

(d)

Figure 1.12: Born term diagrams for pion electroproduction. (a) t-channel (pion pole) mechanism, (b) s-channel (nucleon pole), (c) u-channel (crossed nucleon pole), (d) contact term required to restore gauge invariance.

The Born exchange amplitude is expressed as a product of the probability of a virtual pion interacting with a virtual photon of particular momentum and the probability to encounter a virtual pion with that momentum. The first factor corresponds to the pion form factor $F_{\pi}\left(Q^{2}\right)$ whereas the second factor is described by $\mathrm{g}_{\pi N N}$, the $\pi N N$ coupling. In the pole approximation the pion form factor is 
determined through the relation,

$$
\sigma_{L} \approx \frac{-t Q^{2}}{\left(t-m_{\pi}^{2}\right)^{2}} g_{\pi N N}^{2}(t) F_{\pi}\left(Q^{2}, t\right)
$$

\section{Regge Models}

Another type of model is based on Regge theory, which describes pion electroproduction in terms of the exchange of a family of particles. A Regge model for pion electroproduction developed by Vanderhaeghen, Guidal and Laget (VGL, [11]) models the exchange of $\rho$ and $\pi$ like particles by replacing the pole-like Feynman propagators, $1 /\left(t-m^{2}\right)$, of Born term models with Regge propagators accounting for the exchange of a family of particles with the same internal quantum numbers. The VGL Regge model for pion electroproduction is an extension of a gauge invariant Regge trajectory exchange model, which gives a good description of pion photoproduction data at high energies and low momentum transfer $\left(-t<2(\mathrm{GeV} / \mathrm{c})^{2}\right)$. The model has no free parameters, since the coupling constants at the vertices are well known from studies in the resonance region. The extension to electroproduction is achieved by introducing a monopole pion form factor,

$$
F_{\pi}\left(Q^{2}\right)=\frac{1}{1+\frac{Q^{2}}{\Lambda_{\pi}^{2}}}
$$

where $\Lambda_{\pi}^{2}$ is effectively the only free parameter. It should be noted that the $\rho \pi \gamma$ form factor is a free parameter as well, but does not influence the t-dependence of the longitudinal electroproduction cross section at small momentum transfers, 

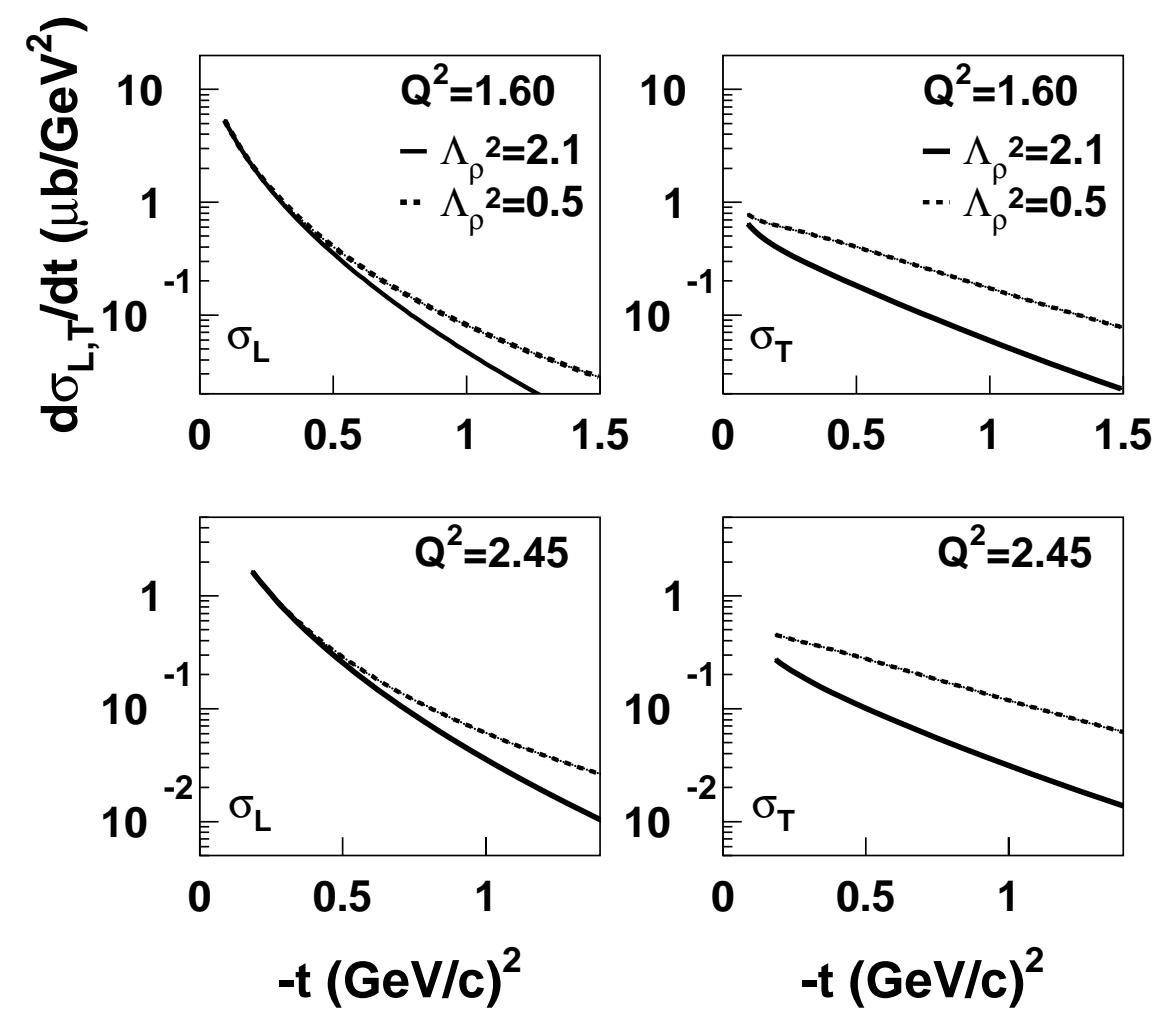

Figure 1.13: Longitudinal and transverse cross sections calculated in the VGL Regge model for different values of $\Lambda_{\rho}^{2}$. While the longitudinal term is not affected at low $-t$, the transverse term changes significantly.

$-t$. The sensitivity to the $\rho$ exchange Regge trajectory can be estimated from a comparison of the transverse electroproduction cross sections. The implementation of gauge invariance in the VGL Regge model by adding a nucleon s-pole term to the Regge amplitude has recently been criticized by Obukhovsky et al. [58]. The authors suggest that gauge invariance is guaranteed provided that the full sum of s-channel resonances included is equivalent to the full sum of Regge trajectory exchanges. Obukhovsky et al. use a constituent quark model approach to model the contribution of $s$-pole resonances including a nonlocal extension of the contact term. To this end, Obukhovsky et al. introduce an additional strong form factor to 
account for the $s$ - and $u$-channel quark transition amplitudes.

$$
F_{\pi}\left(Q^{2}\right) \approx F_{q}\left(Q^{2}\right) \times F_{\pi q q}\left(Q^{2}\right)
$$

where $F_{q}\left(Q^{2}\right)$ and $F_{\pi q q}\left(Q^{2}\right)$ denote the electromagnetic and strong form factors in the pole process $\left(\gamma^{*}+q \rightarrow q+\pi\right)$. The prediction of the $t$-dependence of the cross section contains the common effect of all pole contributions and is intrinsically gauge invariant.

\subsubsection{Competing Reaction Channels}

The extraction of $F_{\pi}$ relies on the dominance of the $\pi$ exchange diagram to the longitudinal cross section (t-channel/pole dominance). In order to test the pole dominance of the experimentally determined longitudinal cross section, the longitudinal ratio $\pi^{-} / \pi^{+}$in deuterium can be used. In exclusive pion electroproduction from deuterium the virtual photon state is a linear combination of the virtual photon isoscalar $(I=0)$ and isovector $(I=1)$ isospin states. In terms of the isoscalar and isovector amplitudes $\left(A_{s}\right.$ and $\left.A_{v}\right)$ the longitudinal $\pi^{-} / \pi^{+}$ratio can be expressed as

$$
\frac{\sigma\left(\gamma^{*} n \rightarrow \pi^{-} p\right)}{\sigma\left(\gamma^{*} p \rightarrow \pi^{+} n\right)}=\frac{\left|A_{v}-A_{s}\right|^{2}}{\left|A_{v}+A_{s}\right|^{2}}
$$

where G-parity conservation requires the pole term to be purely isovector, whereas both isoscalar and isovector contributions are allowed for the other Born terms. A longitudinal $\pi^{-} / \pi^{+}$ratio of unity can be interpreted as a necessary but not 

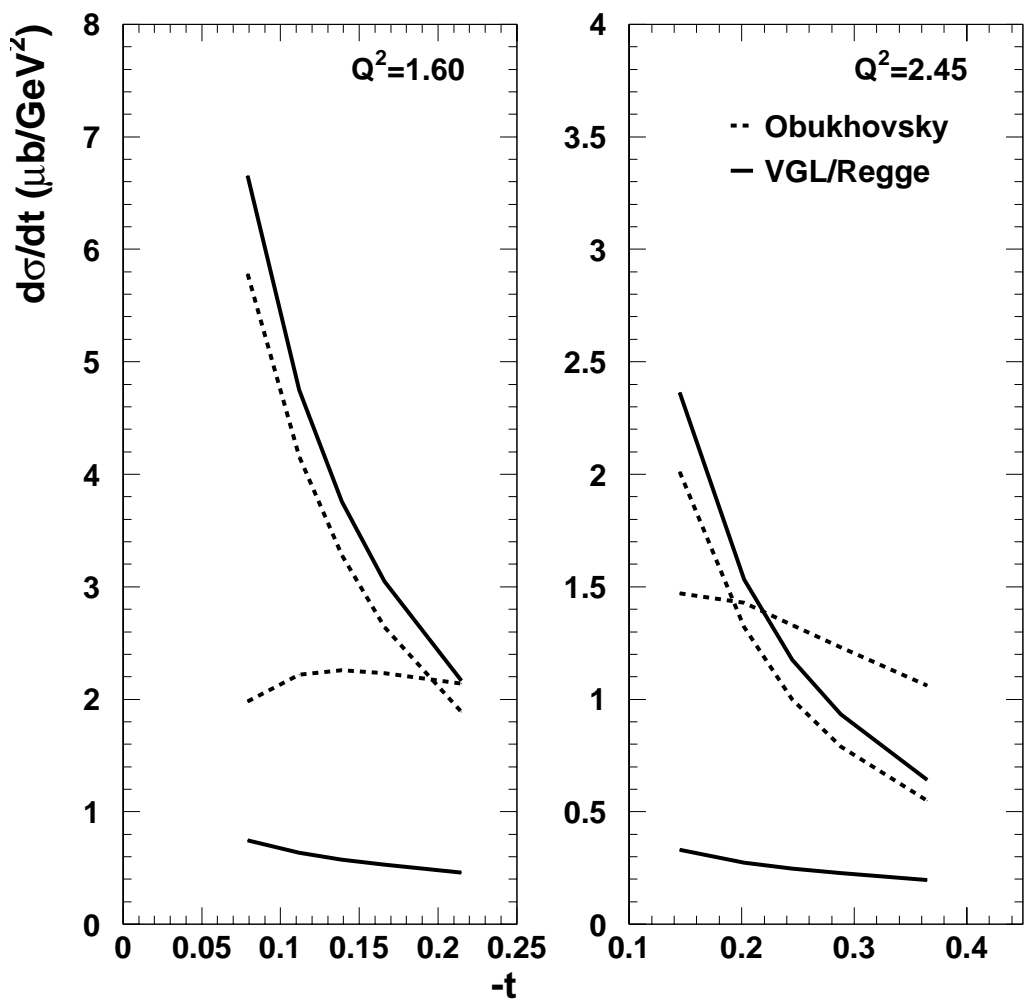

Figure 1.14: Pion electroproduction cross section calculations for $F_{\pi}$-2 kinematics from the VGL/Regge model ([11] and a quark model prediction by Obukhovsky et al. [58]. Both models provide a similar good description of the expected t-dependence of the longitudinal cross section for $\Lambda_{\pi}^{2}=0.54(\mathrm{GeV} / \mathrm{c})^{2}$. The quark model prediction for the transverse piece of the cross section exceeds the VGL/Regge prediction by a factor of 2 .

sufficient condition for the absence of isoscalar background. In particular, at least three cases have to be considered for equation 1.25: 1) The ratio is consistent with unity and the pole process is the dominant contribution, 2) The ratio is consistent with unity, but diagrams $1.12(\mathrm{~b}), 1.12(\mathrm{c})$ and 1.12(d) contribute, and 3) The ratio is not consistent with unity and the pole process does not dominate. The second case results from particular combinations of relative phase factors of the complex amplitudes resulting in a ratio consistent with unity even if the isoscalar contribution 
is nonzero. The absolute isoscalar contribution is unclear in this case. Therefore, a ratio of the longitudinal $\pi^{-} / \pi^{+}$ratio consistent with unity cannot alone be taken as an indication of pole dominance in a measurement of longitudinal cross sections.

As $-t$ increases, the probability for the virtual photon to interact with individual quarks increases. In the limit of large $-t$, the transverse $\pi^{-} / \pi^{+}$ratio is expected to approach a value of $1 / 4$, the square of the charge ratio of the participating quarks. Data from [56] confirm this supposition. In this experimental study, $\sigma_{L}$ and $\sigma_{T}$ were extracted for both reactions $\gamma n \rightarrow \pi^{-} n$ and $\gamma p \rightarrow \pi^{+} n$ allowing for a separate extraction of the longitudinal $\left(R_{L}\right)$ and transverse $\left(R_{T}\right)$ ratios. It was found that $R_{L}$ is consistent with unity over the entire range in $-t$, while $R_{T}$ decreases as a function of $-t$.

\subsection{Overview of the Experiment}

The goal of the experiment E01-004 $\left(\mathrm{F}_{\pi}-2\right)$ [59] discussed here was the measurement of the longitudinal and transverse cross sections via pion electroproduction from hydrogen and deuterium for the purpose of extracting the charged pion form factor assuming pole dominance. The experiment was carried out in Hall $\mathrm{C}$ at Jefferson Lab in July and August of 2003. Pions and electrons were detected in coincidence in magnetic focusing spectrometers. The charged pions were detected in the High Momentum Spectrometer (HMS), while the scattered electrons were detected in the Short Orbit Spectrometer (SOS).

Table 1.1 summarizes the kinematics of this and the previous $F_{\pi}$ measurements. The data were taken at four different beam energies and two values of $Q^{2}$. 


\begin{tabular}{ccccc}
\hline Experiment & $\begin{array}{c}Q^{2} \\
(\mathrm{GeV} / \mathrm{c})^{2}\end{array}$ & $\begin{array}{c}W \\
(\mathrm{GeV} / \mathrm{c})\end{array}$ & $\begin{array}{c}|-t| \\
(\mathrm{GeV} / \mathrm{c})^{2}\end{array}$ & $\begin{array}{c}E_{e} \\
(\mathrm{GeV} / \mathrm{c})\end{array}$ \\
\hline $\mathrm{F}_{\pi^{-}}$ & $0.6-1.6$ & 1.95 & $0.03-0.150$ & $2.445-4.045$ \\
$\mathrm{~F}_{\pi^{-}}$ & $1.6,2.5$ & 2.22 & $0.093-0.189$ & $3.779-5.246$ \\
\hline
\end{tabular}

Table 1.1: Kinematic settings measured in $F_{\pi}-1$ and $F_{\pi}-2$.

In order to attain full coverage in $\phi_{\pi}$ charged pions were detected in parallel kinematics (along the direction of momentum transfer, $q$ ) and at \pm 4 degrees off the direction of momentum transfer. For each $Q^{2}$, data were taken for two values of the virtual photon polarization, $\epsilon$. All data were taken at a fixed center of mass energy, $W=2.22 \mathrm{GeV}$. The following chapter will give an overview of the experimental apparatus. 
Chapter 2

Experimental Apparatus

\subsection{Overview}

The $\mathrm{F}_{\pi}-2$ experiment was performed in Hall $\mathrm{C}$ at Thomas Jefferson National Accelerator Facility (Jefferson Lab) previously known as CEBAF (Continous Electron Beam Accelerator Facility). The data were acquired in July and August of 2003. The unpolarized electron beam from the Jefferson Lab accelerator was incident on a $4 \mathrm{~cm}$ liquid hydrogen or deuterium target located under vacuum in a scattering chamber. Four beam energies were used during $\mathrm{F}_{\pi}-2: 3.778,4.709,4.210$ and 5.246 $\mathrm{GeV}$ with currents between 40 and $90 \mu \mathrm{A}$ depending on the particular target. The scattered electrons were detected in the Short Orbit Spectrometer (SOS) and the coincident electroproduced pions were detected in the High Momentum Spectrometer (HMS). The individual particle detectors were located in concrete-shielded huts in either spectrometer. The signals from the detectors were sent from the experimental hall to remote electronics located in the Hall $\mathrm{C}$ counting house where the data were processed and recorded by the data acquisition system. This chapter describes the details of the experimental apparatus and data acquisition used during $\mathrm{F}_{\pi}-2$.

\subsection{Accelerator}

Jefferson Lab is a medium energy nuclear facility, which consists of a "continous wave" electron accelerator and three experimental halls (A, B and C), that use the electron beam provided to study different aspects of nuclear physics. 


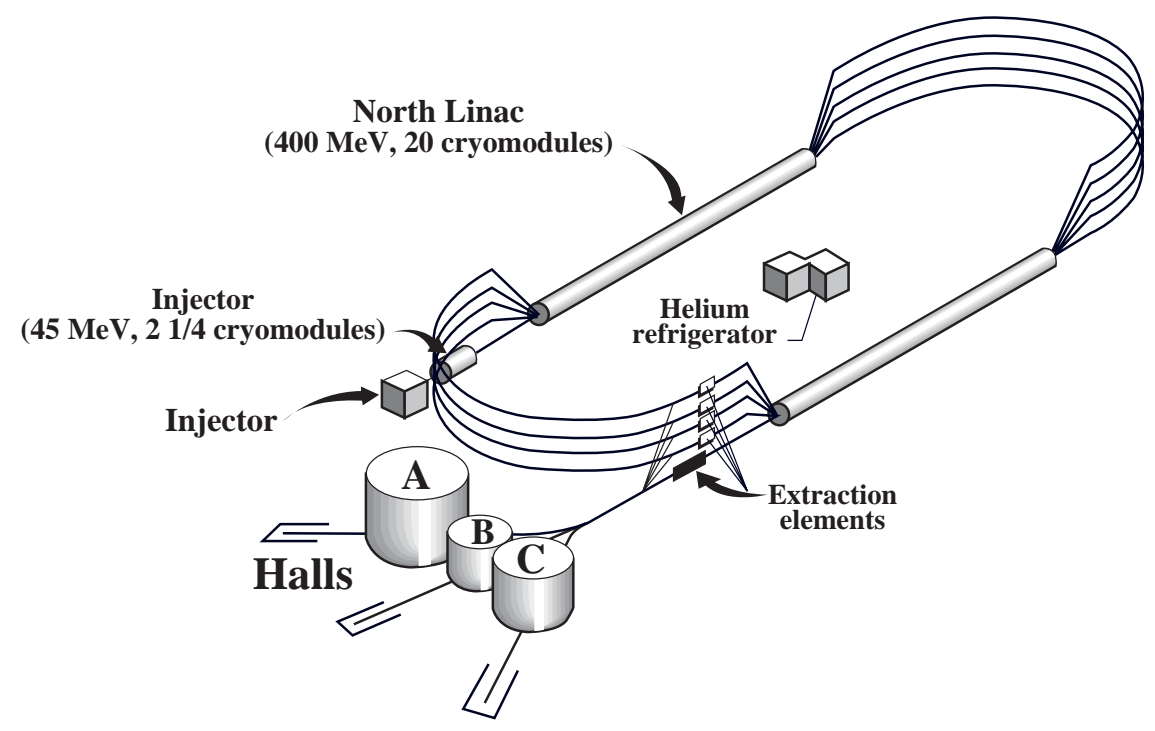

Figure 2.1: Schematic representation of the JLab site.

The Jefferson Lab accelerator is a high intensity, continous wave (CW), superconducting radiofrequency $(\mathrm{RF})$ electron accelerator operating at a fundamental frequency of $1497 \mathrm{MHz}$. A schematic of the site layout of the accelerator is shown in Figure 2.2. Electrons are accelerated to an energy of $45 \mathrm{MeV}$ (for a maximum energy of $4.045 \mathrm{GeV}$ ) and sent to the North Linac where they gain an additional energy of $400 \mathrm{MeV}$ through acceleration through superconducting RF (radio frequency) cavities. From the North linac the electron beam is bent through the east arc and guided through the south linac where it gains another $400 \mathrm{MeV}$ in energy. At this stage the beam can either be sent to the Beam Switch Yard where it can be distributed to the three experimental halls or recirculated through the west arc for an additional pass through the linacs. For each additional pass the electron beam acquires another $800 \mathrm{MeV}$ of energy. The beam can be recirculated up to five times before it is sent to any of the experimental halls. Currently the accelerator is 
capable of delivering continous electron beam of maximum energy $\approx 6 \mathrm{GeV}$. A linac consists of 20 cryo modules, each of which contains eight superconducting niobium cavities cooled by liquid helium at $2 \mathrm{~K}$. The same linacs are used for the acceleration in each circulation. However, the beams from different passes have their own beam pipes in the recirculating arcs and steering magnets allowing each experimental hall to run simultaneously at different energies. All halls can simultaneously receive the maximum energy beam.

The CW beam is not continous, but contains an intrinsic mircostructure. The accelerator produces short beam pulses $\approx 2$ ps long [60], which occur at a frequency of $1497 \mathrm{MHz}$ as a result of the RF power used in the resonating cavities [61]. Every third pulse is delivered to each of the experimental halls resulting in a frequency of $499 \mathrm{MHz}$, or one pulse every $2 \mathrm{~ns}$, beam structure for each hall. The beam pulses are separated after each linac pass by means of RF separators at the Beam Switch Yard. It should be noted that at this rate the beam delivery can effectively be considered continous. For a coincidence experiment like $\mathrm{F}_{\pi}-2$, the continous beam property is important when considering the accidental coincidence rate. Since the accidental coincidence rate is inversely proportional to the beam duty factor, the accidental coincidence rate would be larger at pulsed beam accelerators with lower duty factors. Further information on the accelerator design can be found in [61].

\subsection{Hall C}

A schematic layout of experimental Hall $\mathrm{C}$ is shown in Figure 2.7. The hall has a nearly circular geometry with a diameter of $32 \mathrm{~m}$. A large fraction of the exper- 


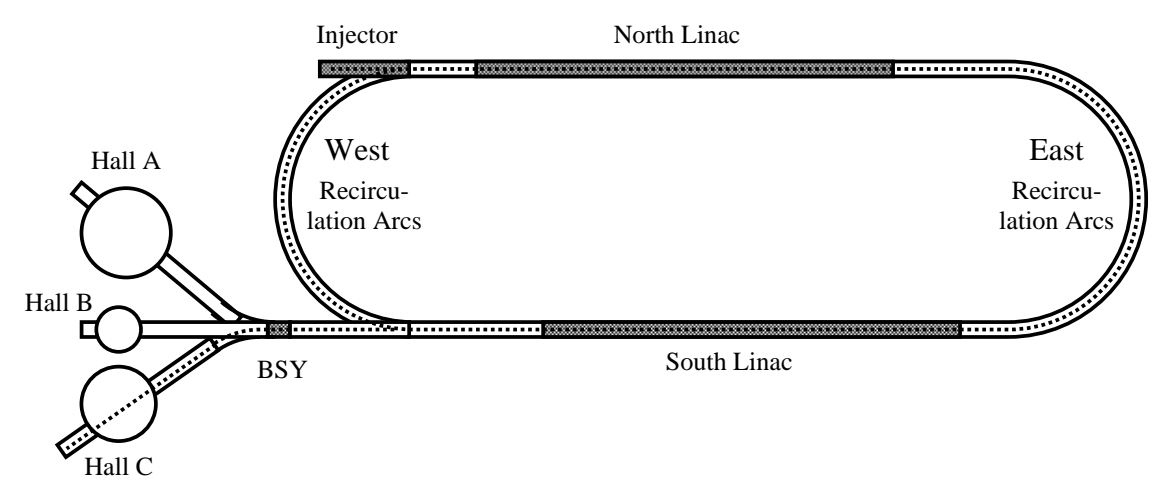

Figure 2.2: Schematic representation of the JLab accelerator.

imental hall is located underground and is well shielded to contain radiation. The standard Hall C apparatus consists of two magnetic focusing spectrometers. The High Momentum Spectrometer (HMS) optics configuration consists of three superconducting quadrupoles followed by a dipole and has a pathlength of approximately $26 \mathrm{~m}$ from the target to the focal plane. In contrast, the Short Orbit Spectrometer (SOS) optics consists of three resistive magnets and has a pathlength of $10 \mathrm{~m}$ appropriate for the detection of short-lived particles. The momentum resolutions of the HMS and SOS are better than $1 \times 10^{-3}$ and the horizontal angular resolutions are better than 2 mrad. The design maximum central momentum for the SOS is $1.74 \mathrm{GeV} / \mathrm{c}$. The standard instrumentation in Hall $\mathrm{C}$ has been used successfully for a variety of experiments requiring high luminosity.

\subsection{Beamline}

\subsubsection{Beam Position Monitors}

Beam Position Monitors (BPM) are used to monitor the position of the beam in the Hall $\mathrm{C}$ arc and the beamline. These monitors consist of resonating cavities 
with a fundamental frequency to match both the $1497 \mathrm{MHz}$ accelerator beam pulse frequency and the $499 \mathrm{MHz}$ pulse frequency in Hall C. Each cavity has four antennae which are rotated by $45^{\circ}$ with respect to the horizontal and vertical axes to minimize synchrotron damage. The amplitude of the signal picked up from the fundamental frequency by each antenna allows for determining the relative position of the beam $[62]$.

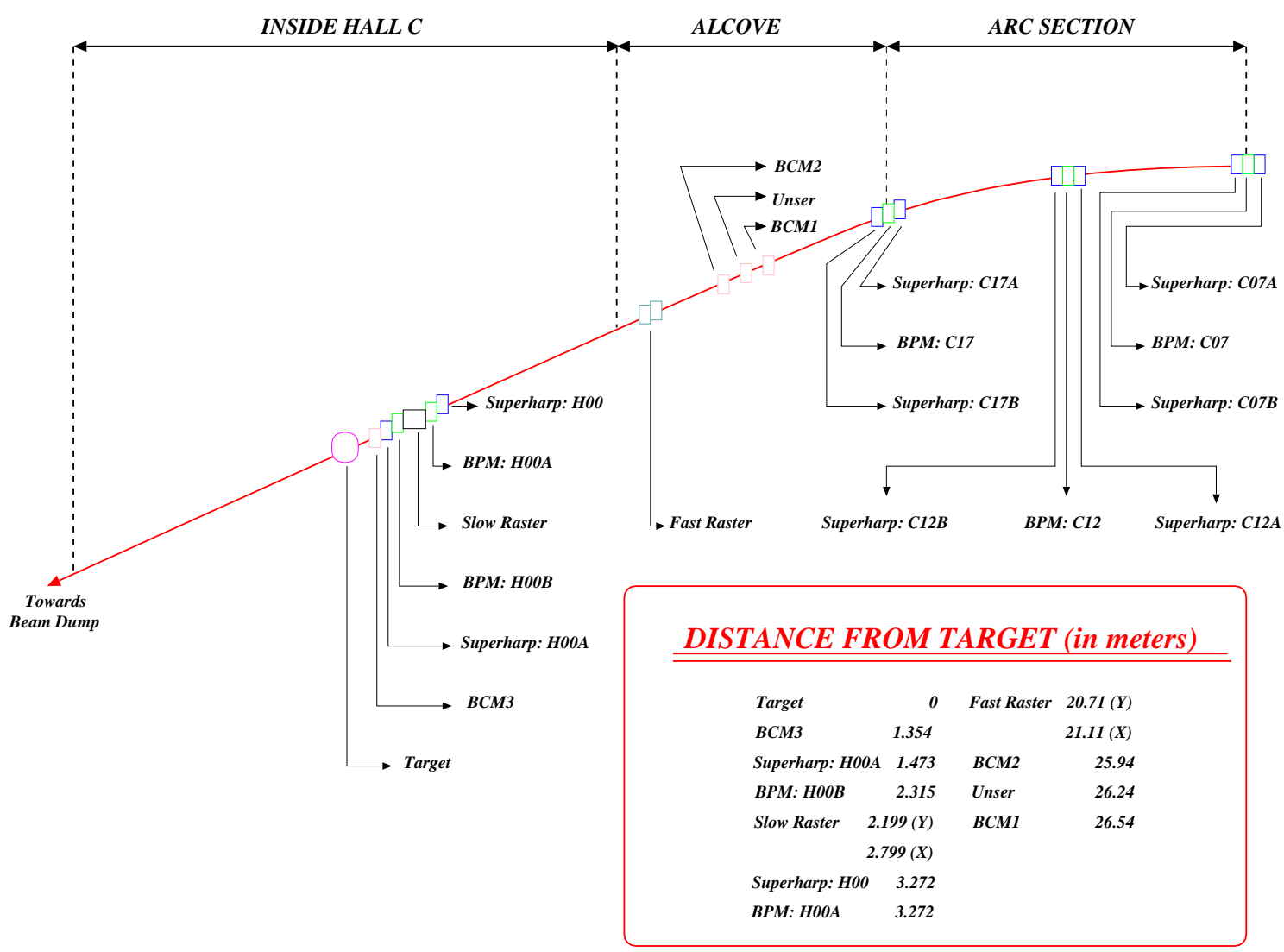

Figure 2.3: Schematic representation of the instrumentation along the Hall $C$ beamline.

The BPMs in the Hall C arc are typically used for guiding the beam. However, the BPMs closest to the Hall C target (H00A, H00B, H00C) were also monitored to ensure consistency of the beam position on the target. The nominal beam position 
was set on the BPMs based on information from spectrometer optics data and varied for each of the four beam energies. It should be noted that the BPM coordinates do not represent the absolute position of the beam and are chosen based on the requirement of simultaneous mid-plane symmetry in both spectrometers. Given the location of the BPMs (see Figure 2.3), the beam position at the actual target location can be determined from the projection of the combination of any pair of BPMs. During the experiment BPM C was determined to be not reliable so that for all subsequent calculations BPMs A and B were used. The typical size of the variation of the position at the target was less than $0.5 \mathrm{~mm}$.

The beam profile is monitored using the superharps along the Hall $\mathrm{C}$ arc and beamline. The harp system consists of a nonstationary frame and two vertically and one horizontally oriented wires. During a "harp scan" the differently oriented wires move across a low current beam. The signals generated at each wire as they

are intercepted by the beam are recorded by an analog to digital converter. The corresponding position of the wire intercepted is determined by a position encoder. The superharp system is an upgrade to the harp system including absolute position readout electronics, a dual beam profile detection system with two analog pick-up channels and a vibration-free support system. The harp system and its operation are described in more detail in reference [63].

\subsubsection{Beam Energy Measurement}

The energy of the electron beam in Hall $\mathrm{C}$ is measured using the deflection of the electron beam in a known magnetic field in the Hall $\mathrm{C}$ arc. This measure- 
ment makes use of the fact that an electron moves on a circular trajectory in a constant magnetic field where its radius depends on the strength of the magnetic field and the electron's momentum. The arc method uses the arc magnets as a type of spectrometer and the superharps to measure the deflection of the beam in the section of the beam line between the Beam Switch Yard (BSY) and the hall entrance. Figure 2.4(b) illustrates the layout of the arc measurement setup.

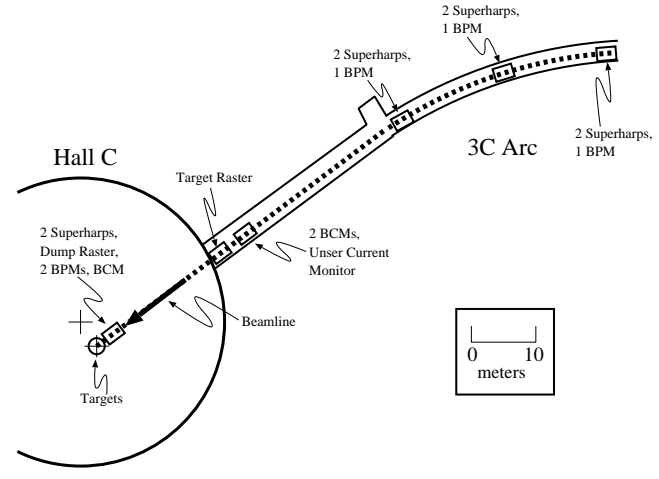

(a) Hall C Arc

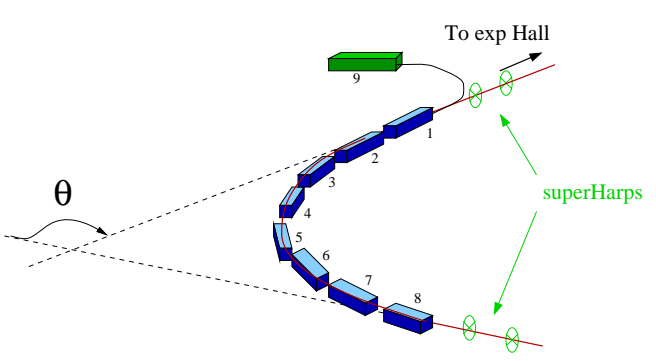

(b) Arc Energy Measurement

Figure 2.4: Beam Line Elements.

This measurement cannot be performed simultaneously with the regular data taking, since it requires that all focussing elements be turned off and degaussed. The beam position and direction at the entrance, middle and the exit of the arc are measured using the high resolution wire sensors (superharps). The bend angle of the Hall $\mathrm{C}$ arc, $\theta_{\text {arc }}$, was measured to be $34.3^{\circ}$. The beam is then steered so that the central trajectory and the beam energy can be determined from the electron momentum using

$$
p=\frac{e}{\theta_{\text {arc }}} \int B d l,
$$


where $e$ is the electron charge, $\theta_{\text {arc }}$ is the arc bend angle and $\mathrm{B}$ is the magnetic field in the dispersive elements. The extraction of the beam energy from the field integral requires the knowledge of the magnetic fields in the arc dipoles. For this reason, one of the dipoles in the Hall $\mathrm{C}$ arc has been field-mapped as a function of current. The remaining eight dipoles are calibrated relative to the reference dipole assuming similar field maps. Using the value of the field integral the beam energy

can be determined with a precision of $\frac{\delta p}{p} \approx 5 \times 10^{-4}$ [64]. A detailed description of the beam energy measurement using the arc method is available in reference [65].

\subsubsection{Beam Current Monitors}

The electron beam current delivered to the hall is measured by two Beam Current Monitors (BCM). The primary BCMs (BCM1 and BCM2) are cylindrically shaped wave guides tuned to the frequency of the beam. In particular, the geometry of the cavities was designed to be excited by the $T E M_{010}$ mode of the electron beam pulse frequency. The advantage of the $T E M_{010}$ mode is that its magnitude changes very slowly with respect to the position of the beam within the cavities. The output voltage levels of the wave guides is proportional to the beam current when the wave guides are tuned to the frequency of the beam.

The current monitor cavities are sensitive to temperature. Due to expansion and contraction in response to changing temperatures the resonant frequency of the cavities is modified resulting in a decrease of the measured power and therefore in the current measurement. In order to minimize temperature fluctuations, the temperature is stabilized by thermally insulating the beam monitor cavities at a 
temperature of $43.3^{\circ} \mathrm{C}$. The cavity temperature was checked during each shift and found to be stable within $\pm 0.2^{\circ} \mathrm{C}$. It should be noted that the temperature of the readout electronics can also affect the current measurement. In order to minimize this effect due to temperature variations the electronics room was maintained at a nearly constant temperature throughout the experiment.

Both BCM1 and BCM2 generally have a reasonable gain stability as a function of time. However, to minimize drifts in the gain both BCMs are calibrated to an absolute standard device at regular intervals. The calibration is performed using an Unser current monitor [66], which is a parametric DC current transformer. The Unser monitor has an extremely stable gain, but suffers from large drifts in the offset on short time scales. Thus, the Unser monitor cannot be used alone to measure the beam current reliably on a run to run basis. The resonant cavity BCMs were calibrated by taking dedicated runs with periods of no beam (thus establishing the Unser zero/baseline) interspersed with periods of beam at various currents. During $\mathrm{F}_{\pi^{-}}$, the currents ranged from $10 \mu \mathrm{A}$ to $110 \mu \mathrm{A}$ with stepsize $10 \mu \mathrm{A}$. The gain of the BCMs was then determined by comparing to the Unser monitor. The BCMs are generally stable enough so that calibrations have to be performed only infrequently during the experiment. The run-to-run uncertainty in the current as measured by BCM1 and BCM2 is estimated from a global analysis of the fit residuals added in quadrature with the average drift between calibrations and found to be on the order of $0.2 \%$ at $100 \mu \mathrm{A}$. Considering in addition the normalization uncertainty from the Unser, which is estimated to be $0.4 \%$ results in an absolute uncertainty for the charge measurement of $0.5 \%$. 


\subsubsection{Modification to Beamline}

For the $\mathrm{F}_{\pi}-2$ experiment a small diameter beam pipe ( $\mathrm{d}=2$ inches) was installed downstream of the target to allow for data taking at the smallest possible separation angle between the beam line and the Hall $\mathrm{C}$ spectrometers. In this particular geometry (at large SOS central angles) the beam pipe is susceptible to magnetic fields from an unshielded edge of the SOS dipole magnet. The presence of these magnetic fields was confirmed prior to the experiment from measurements at a momentum setting of $1.74 \mathrm{GeV} / \mathrm{c}$. The dominant fields are parallel to the dipole yoke and oriented along and perpendicular to the spectrometer axis. The contribution from magnetic fields vertical to the magnet yoke and perpendicular to the spectrometer axis are $20 \%$ smaller [67].

The main concern of the beam deflection was the location of the beam at the diffuser at the exit of the hall. A beam deflection exceeding the radius of the critical aperture could result in significant damage to one of the flanges in the Hall $\mathrm{C}$ beam dump due to excessive energy deposition. In case of significant damage contaminated water from the Beam Dump Cooling system could drain directly into Hall C and prevent experiment related activities for an indetermined time. The deflection of the beam was calculated for different kinematic settings using magnetic field map data. In the calculation, a SOS momentum of $1.74 \mathrm{GeV} / \mathrm{c}$ and a beam energy of $5 \mathrm{GeV}$ were assumed. The deflection at the smallest angle for $\mathrm{F}_{\pi^{-}}-2$ kinematics was determined to be $\pm 4 \mathrm{mrad}$ from the target center [68]. The vertical deflection of the beam at the diffuser was addressed with magnetic shielding of the downstream beam pipe. In 
order to reduce the value of the field integral and corresponding beam deflection two layers of co-netic AA foil were installed around the beam pipe. Detailed tests of the beam deflection with the modified beam pipe entailing measurements at SOS angles between 22 and $30^{\circ}$ and the SOS in full saturation confirmed adequate suppression of beam deflection within acceptable boundaries [68].

\subsection{Targets}

The Hall C target system contains a three-loop cryogenic target stack mounted together with optics and "dummy" targets on a target ladder enclosed in an evacuated scattering chamber. The solid target ladder consists of five carbon and two aluminum foils at different $\mathrm{z}$-positions $(\mathrm{z}=0, \mathrm{z}= \pm 2 \mathrm{~cm}, \mathrm{z}= \pm 4 \mathrm{~cm}$, and $\mathrm{z}= \pm 7 \mathrm{~cm})$ along the beam direction. The two aluminum foils situated at $\mathrm{z}= \pm 2 \mathrm{~cm}$ constitute the "dummy target" used to measure the aluminum cell wall contribution to the cryotarget yields. The dummy target is approximately seven times thicker than the nominal thickness of the cryotarget cell walls. The remaining solid carbon foils are used with beam incident on two or five ("quintar") foils simultaneously for the purpose of calibrating spectrometer optics properties. The average energy deposition in the cryogenic targets is relatively large $\left(5 \mathrm{MeV} \mathrm{cm}^{2} \mathrm{~g}^{-1}\right)$ while the diameter of the incident electron beam is relatively small. To minimize the damage from local heating during the experiment, the beam was rastered to a $2 \times 2 \mathrm{~mm}$ profile to distribute the energy in a more uniform manner over the cryotarget volume. It should be noted that the rastering profile consisted of a constant structure in contrast to the sinusoidal rastering profile used in previous experiments. A more detailed 
description of the fast raster system can be found in reference $[69,70]$

\subsubsection{Cryogenic Targets: Hydrogen and Deuterium}

The liquid hydrogen and deuterium target cells are vertical flow, round (tuna can), $4 \mathrm{~cm}$ target cells, each occupying one of the three available loops. During $\mathrm{F}_{\pi^{-}}$, loop 2 contained liquid hydrogen and loop 3 liquid deuterium, while loop 1 was kept empty. Both cryogenic targets use the same coolant supply and are cooled on the cryotarget ladder simultaneously. $15 \mathrm{~K}$ helium is supplied by the End Station Refrigerator (ESR) and the coolant flow to the individual loops is controlled by the target operator using the Joule Thompson (JT) valves. The cryogen is circulated continously through the heat exchanger from the target cell. Low and high power heaters are controlled by a Proportion, Integral and Derivative (PID) feedback system keeping the cryogen at the appropriate operating temperatures of 19K (LH2) and $22 \mathrm{~K}$ (LD2). The PID system regulates the cryogenic target temperature by mimicking the power deposition of the electron beam using high power heaters during low current of beam-off periods. The target fluid moves continously through the heat exchanger to and from the target cell. Figure 2.5 shows a schematic of the cryogenic target assembly. Each target cell is approximately $4 \mathrm{~cm}$ long in the beam direction with cell walls made from Aluminum alloy T6061 and of thickness $\approx 0.0127 \mathrm{~cm}$. The alloy used in manufacturing the Aluminum dummy targets is Al-T7075, a higher strength alloy. Further details on the mechanical aspects of the cryotargets can be found in $[72,73,74]$.

The cryogenic target density and lengths are affected by several quantities 
such as temperature, pressure variation and boiling effects. In general, the cryogenic targets are kept at a nominal operating temperature about $2 \mathrm{~K}$ subcooled below the boiling point. For example, the hydrogen target was kept at a temperature of $19 \mathrm{~K}$ at density of $0.0723 \pm 0.0005 \mathrm{mg} / \mathrm{cm}^{3}$ [72]. The corresponding length of the cell in the cooled-down state is $3.98 \pm 0.01 \mathrm{~cm}$. This value derives from a measurement of the target length at room temperature, corrections for target offset from the center of the beamline and thermal contraction $(0.4 \% \pm 0.2 \%)$ described in more detail in Appendix B. The deuterium target was operated at $22 \mathrm{~K}$ and a density of $0.167 \pm$ $0.001 \mathrm{mg} / \mathrm{cm}^{3}$.

\subsection{Target Thickness}

The cryotarget thickness and associated uncertainties are listed in Table 2.1. The total target thickness is determined using the target cell geometry at operating temperatures in combination with the target density derived from cell temperature and pressure. For both hydrogen and deuterium the cell temperature was kept constant to within $100 \mathrm{mK}$ during the experiment. Given the negligible contribution from uncertainty in temperature the dominant uncertainty in target density is due to the equation of state, and is about $0.5 \%$. The outer diameter of the target cell at room temperature was measured to $0.3 \%$. The thickness of the cell walls was determined to $\pm 0.0013 \mathrm{~mm}[75]$.

Due to the circular shape of the target cells the effective target length as seen by the beam is sensitive to both the size and form of the raster pattern and the central position of the beam from the target center. The reduction of effective target length 


\begin{tabular}{||c|c|c||}
\hline Target & $\begin{array}{c}\mathrm{L}_{\text {target }} \\
(\mathrm{cm})\end{array}$ & $\begin{array}{c}\mathrm{t}_{\text {cryogen }} \\
\left(\mathrm{g} / \mathrm{cm}^{2}\right)\end{array}$ \\
\hline \hline $\mathrm{LH}_{2}$ & $3.918 \pm 0.01$ & $0.283 \pm 0.002$ \\
$\mathrm{LD}_{2}$ & $3.916 \pm 0.01$ & $0.654 \pm 0.005$ \\
\hline
\end{tabular}

Table 2.1: Cryotarget thicknesses, not corrected for beam offset. The cryotarget length, measured at room temperature, is corrected for thermal contraction of the aluminum cell walls and the offset of the cryotarget from the surveyed target position (3.42 $\mathrm{mm}$ ). The final target length is given by $L=2 \cdot \sqrt{R^{2}-d x^{2}}$, where $R$ is the target cell radius corrected for thermal contraction and with the cell walls subtracted, and $d x$ is the beam offset from the target center. The actual target thicknesses for these targets were also corrected for the beam offset from the target center at each kinematic setting (see Figure 2.6). The uncertainty on the target thickness is the quadrature sum of $0.6 \%$ uncertainty on the target length and $0.5 \%$ on the target density.

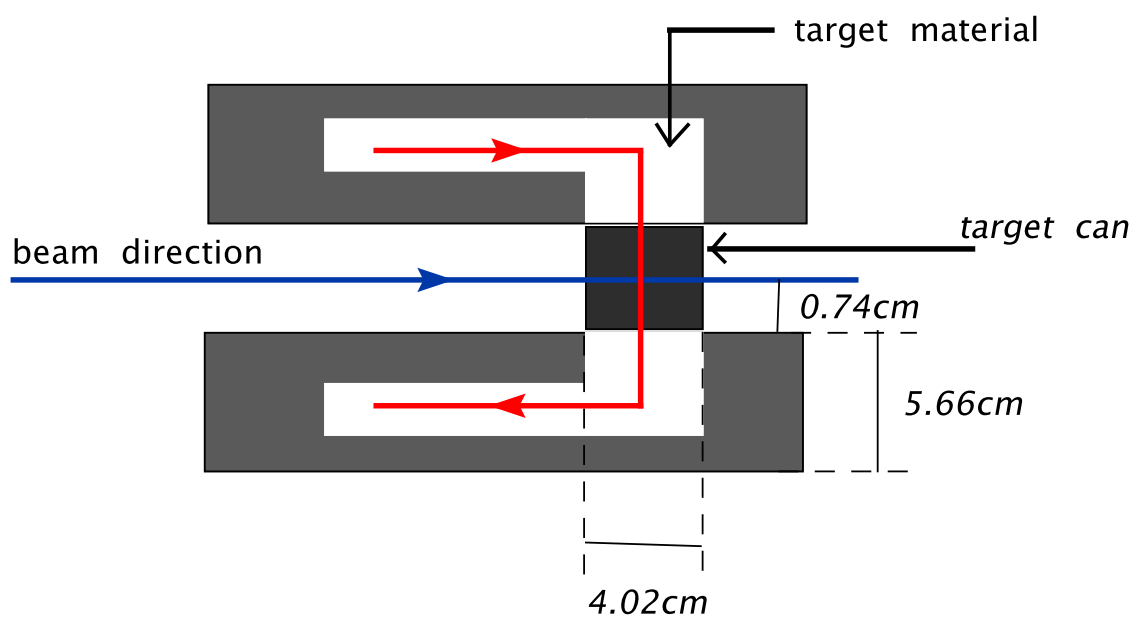

Figure 2.5: Cross section of the cryotarget loop.

due to the constant raster pattern was determined to be $\approx 0.005 \%$. Inital target survey results and measurements of thermal effects like vacuum motion and target cooldown motion indicate that the target cells were on average located at $3.42 \pm 0.5$ $\mathrm{mm}$ (beam right facing downstream) relative to the nominal beamline. Optics data and information from the beam position monitors suggest that the beam was offset between 0.15 and $2.00 \mathrm{~mm}$ in the same direction for the four different kinematic 
settings (Figure 2.6). In the worst case deviation of the beam from the target center the correction of the effective target length is $1.5 \pm 0.05 \%$. The variation in target thickness due to the central beam position between high and low $\epsilon$ settings is $0.2 \%$.

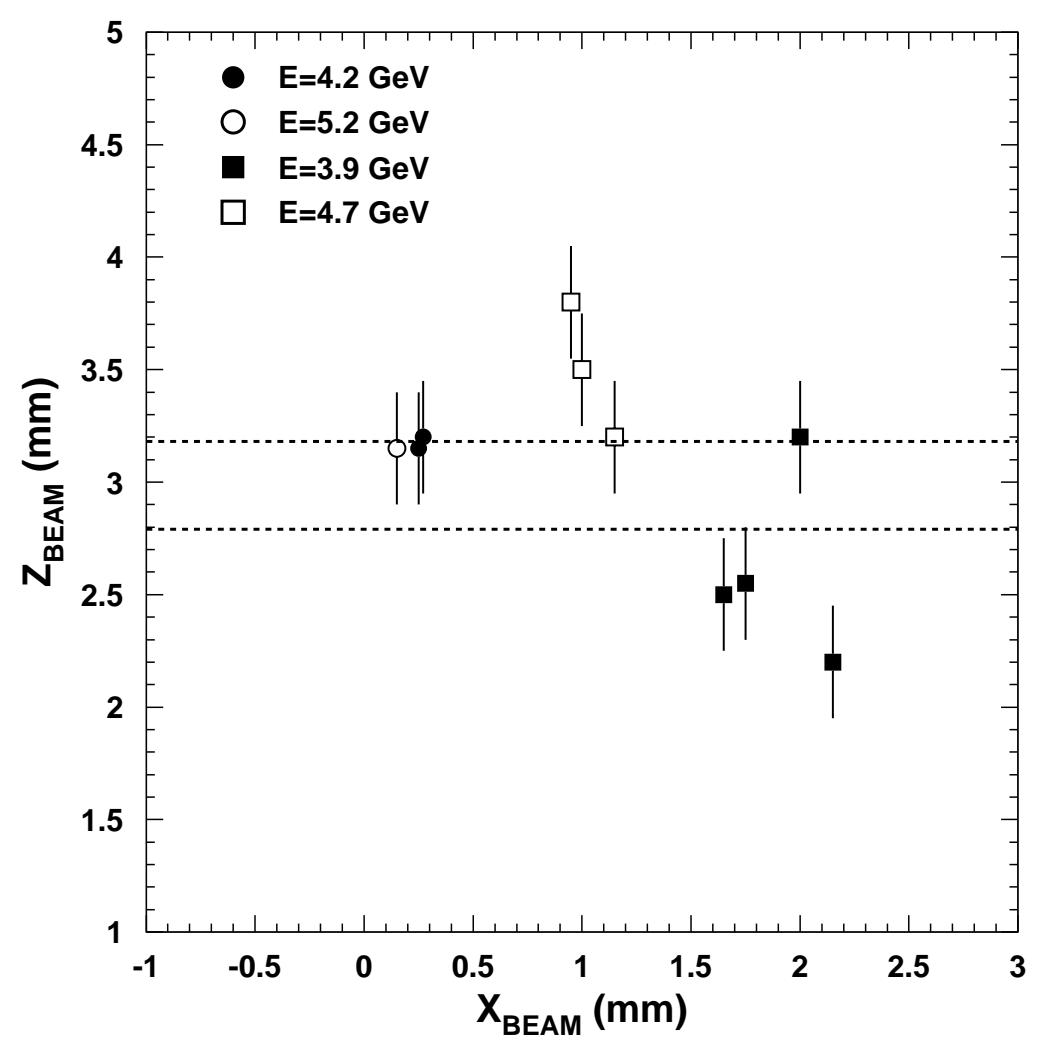

Figure 2.6: Beam position offset with respect to cryotarget center. The vertical axis denotes the location of the cryotarget along the beam in the lab frame. The horizontal axis shows the position of the beam relative to the target center also in the lab frame.

Additional uncertainties to the target thickness are given by purity of the target gas and dynamic effects such as target heating due to energy deposited by the electron beam. To determine the target purity samples of the target materials were examined after the experiment. The purity for both targets was found to be 


\begin{tabular}{||c|c|c||}
\hline Source & $\begin{array}{c}\text { Point-to-point } \\
(\%)\end{array}$ & $\begin{array}{c}\text { Scale } \\
(\%)\end{array}$ \\
\hline \hline Thermal Contraction & - & 0.2 \\
Equation of State & - & 0.5 \\
Cell diameter & - & 0.6 \\
Beam position at target & 0.2 & \\
Target Purity & & $<0.1$ \\
Sum & 0.2 & 0.8 \\
\hline
\end{tabular}

Table 2.2: Cryotarget thickness uncertainties. The random uncertainty is determined by the beam position on target.

$>99.9 \%$ and thus no correction was assigned.

Localized target density fluctuations due to heating effects can have a significant effect on the average density of cryogenic targets. The rastering of the beam reduces local density fluctuations of the liquid targets but cannot eliminate them entirely. The change in luminosity due to beam heating was measured by comparing yields at fixed kinematics as a function of beam current. To account for the net reduction in measured target density due to localized target boiling a correction factor is applied. Taking into account the uncertainty in the beam current, the total uncertainty in the target density is on the order of $0.6 \%$. No significant target density reduction due to localized heating was determined, thus no correction factor is applied. A detailed discussion on the measurment of the target density reduction is given in Appendix B. The composition of the cryotarget uncertainties are listed in Table 2.2. 


\subsection{Spectrometers}

The Hall C spectrometers were designed for the detailed study of the structure of nuclei using both exclusive and inclusive reactions. The core of the experimental equipment are the two magnetic spectrometers used for the detection of the particle of interest. A schematic of the spectrometers is shown in Figure 2.7. Both spectrometers have a relatively large momentum and solid angle acceptance and are equipped with similar and highly versatile detector packages. The Short Orbit Spectrometer (SOS) was designed with a relatively short distance to the focal plane $(\approx 10 \mathrm{~m})$ minimizing decay losses. This characteristic makes the SOS ideally suited to detect short-lived particles such as pions and kaons. However, in this experiment, the SOS was used to detect electrons. The High Momentum Spectrometer (HMS) was built with a larger distance to the focal plane $(\approx 26 \mathrm{~m})$, which makes it more suitable for the detection of high momentum hadrons or electrons. It should be noted that the detector setup allows for both spectrometers to be used to detect hadrons as well as electrons.

\subsubsection{High Momentum Spectrometer (HMS)}

The HMS is a magnetic spectrometer consisting of three quadrupole magnets used as focusing elements and a $25^{\circ}$ vertical bend dipole magnet. The quadrupole magnets are superconducting and are operated in a point-to-point optical tune. The dipole magnet bends in the dispersive direction and determines the central momentum of the spectrometer. The design momentum acceptance of the HMS is 


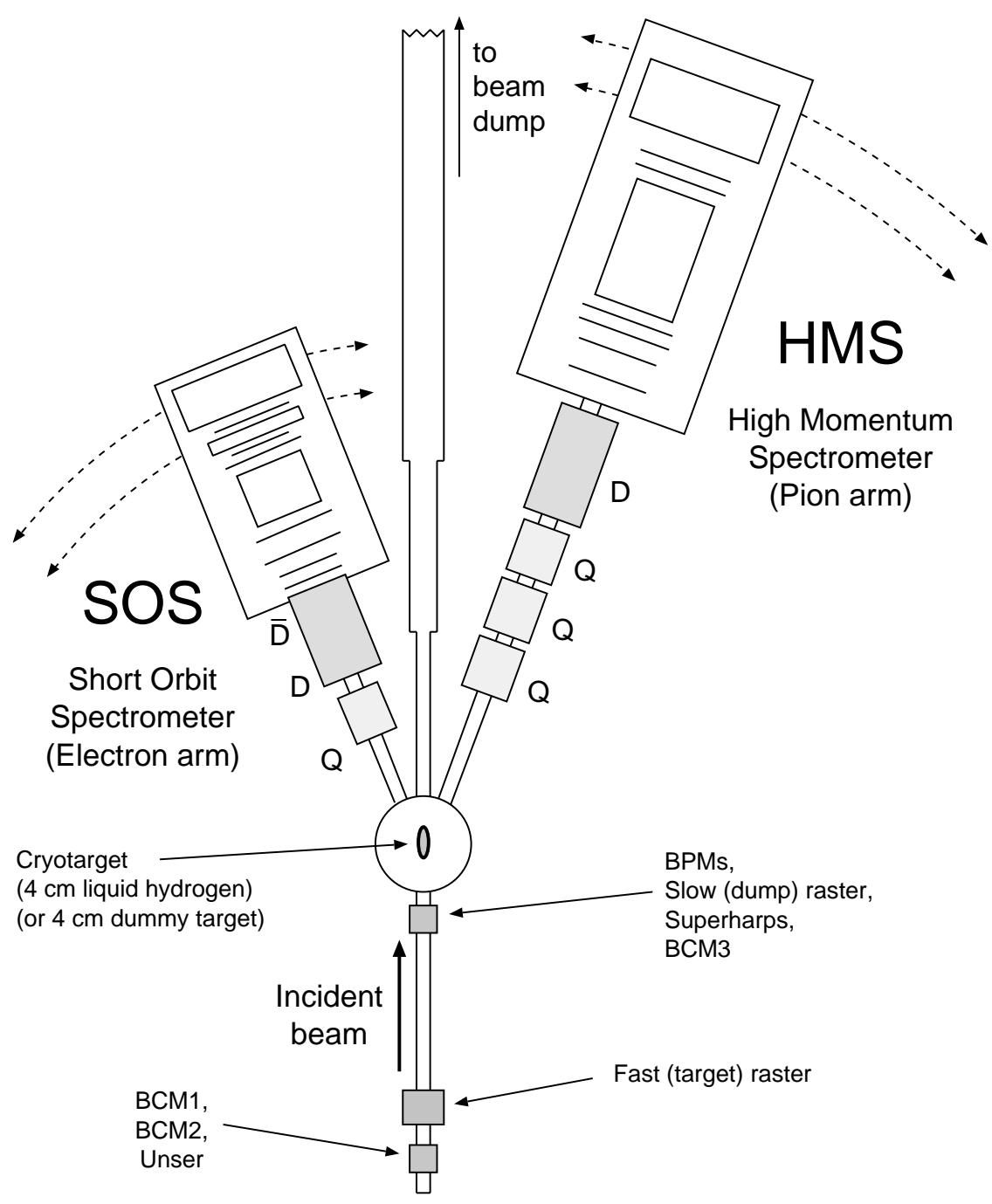

Figure 2.7: Schematic top view of the Hall $C$ spectrometers relative to the target and beamline.

$\approx \pm 10 \%$ ) with a maximum central momentum of $7.4 \mathrm{GeV}$. The detector stack shown in Figure 2.9 is situated in the concrete shielding hut about $26 \mathrm{~m}$ from the pivot.

In order to minimize multiple scattering and to provide thermal insulation, the region between Q1 and the entrance to the spectrometer hut is evacuated. The vacuum region are separated from the surounding environment by vacuum windows. The largest vaccum window in Hall $\mathrm{C}$ is the HMS spectrometer exit window located 
in the detector hut. In the past the spectrometer exit window was composed of mylar. However, the standard window was replaced with a 0.02 inch Titanium window (3.56 radiation lengths) prior to the experiment. A detailed discussion of the Hall $\mathrm{C}$ spectrometer vacuum system and spectrometer vacuum windows can be found in reference [76].

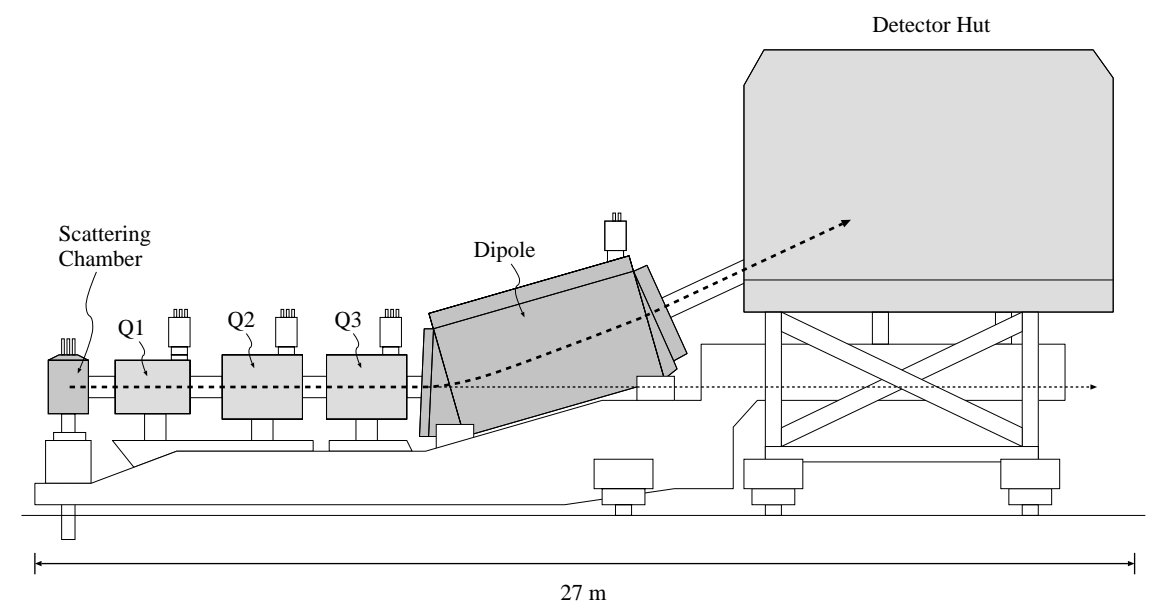

Figure 2.8: Schematic representation of the HMS. The spectrometer consists of three focusing quadrupole magnets and one vertical bend dipole magnet. The distance from the target to the focal plane is $\approx 26 \mathrm{~m}$.
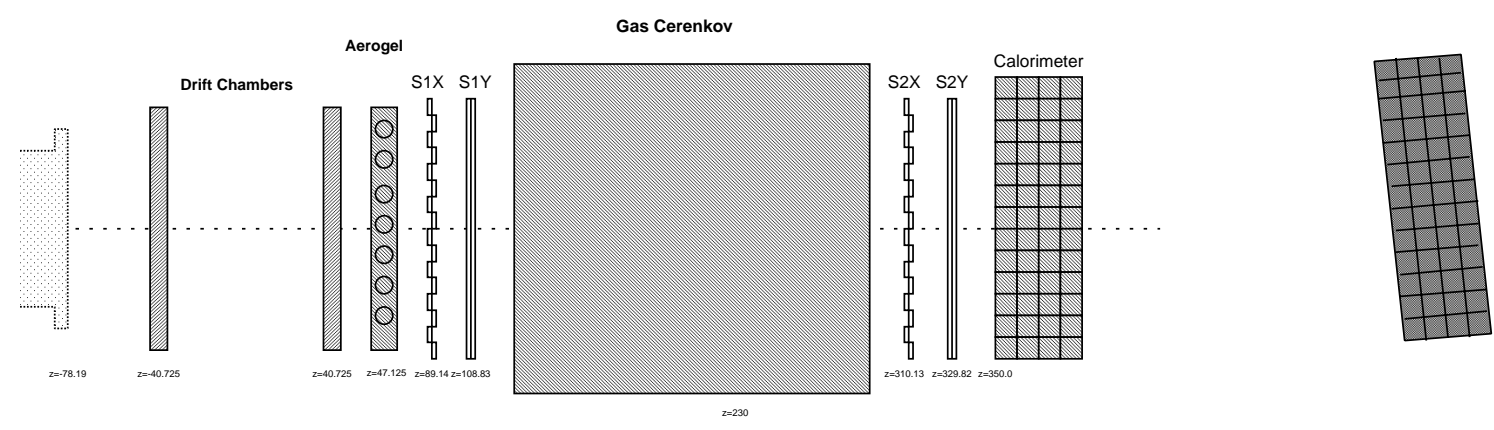

Figure 2.9: Schematic sideview of the HMS detectors. The lead glass calorimeter is tilted as an angle of $5^{\circ}$ relative to the central ray to minimize the loss of particles through the spaces between the calorimeter blocks.

The angular acceptance of the HMS is defined by an octagonal collimator posi- 
tioned between the target and the first quadrupole magnet. The collimator defines a maximum angular acceptance of $6.8 \mathrm{msr}$ in total solid angle. In general, three collimators are available (large, pion and sieve), where the sieve slit collimator is exclusively used for optics calibration. The collimators are designed from $3.175 \mathrm{~cm}$ thick HEAVYMET, which is a machinable tungsten alloy with $10 \% \mathrm{CuNi}$ contamination. Whereas the pion and large collimators are flared along the inside edge to match the particle distribution emanating from the target, the holes in the sieve slit collimator are not flared to avoid particle showers created by incident electrons hitting edges of the sieve holes. With the exception of electrons creating particle showers at flared edges all collimators can be considered as stopping incident electrons.

The setting of the magnets is crucial in the definition of the spectrometer momentum and therefore in the reconstruction of detected particle tracks. The spectrometer momentum is defined by the magnetic field integral over the central trajectory. Assuming that this length is constant, the ratio of the momentum to the magnetic field strength should be constant $\left(\frac{p}{B}=\right.$ constant $)$. Therefore, a deviation from a constant value can be attributed to a magnet offset. Whereas the dipole is set by field using an NMR probe in the magnet, the HMS quadrupole magnets are set by current. To maximize the reproducibility of the quadrupole settings a special procedure was designed. The motivation and general implementation of this procedure can be found in reference [56]. During $\mathrm{F}_{\pi^{-}}-2$ the procedure was implemented as follows: the quadrupoles were ramped to a value exceeding the desired set current by $100 \mathrm{~A}$ and then ramped back down to the required setpoint. To eliminate residual 
fields from previous current settings for polarity changes the magnets were cycled by ramping the current to $800 \mathrm{~A}$ and back to zero. With this particular cycling method a reproducibility of the magnetic fields of one part in $10^{-4}$ was achieved.

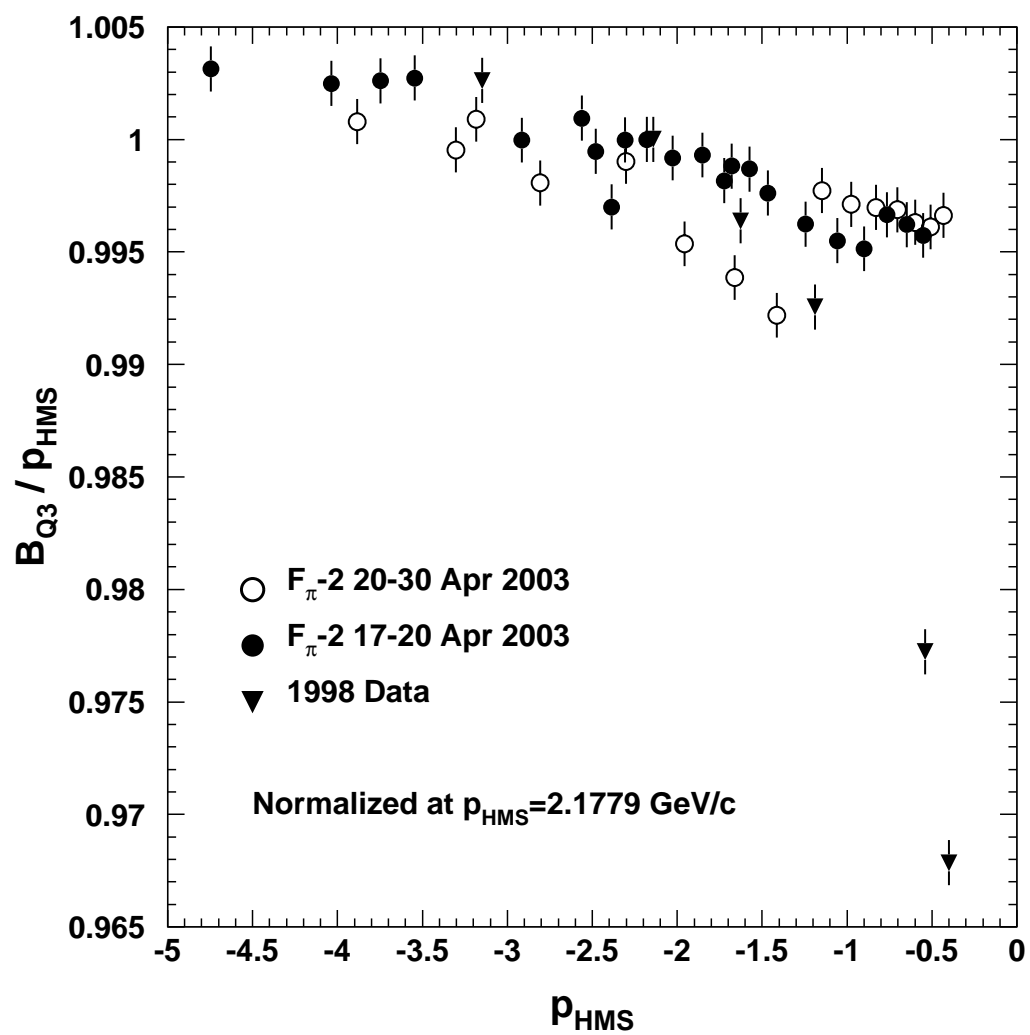

Figure 2.10: $B_{Q 3} / P_{H M S}$ normalized to 1.0 at $P_{H M S}=2.77 \mathrm{GeV} / \mathrm{c}$. In the ideal case, the ratio is expected to be constant as a function of HMS central momentum. The 1998 data were obtained from reference [71]. The data from 2003 are separated into time periods before and after the Hall probes were zeroed.

The quadrupoles are monitored using the power supply readback current and the Hall probes. The magnetic field from the Hall probes is normally used to ensure that the same relative field values result from similar set currents. However, during the experiment it was found that a small but significant offset in the third quadrupole (Q3) set current persists from its discovery in 1998 [55] and the accu- 
racy of the readback current was deemed questionable. An offset in Q3 had been observed previously, and was addressed with a correction to the magnet field setting routine. However, a residual Q3 offset remained observable during $\mathrm{F}_{\pi}-2$ as shown in Figure 2.10. In this analysis the residual Q3 offset was addressed by an ad-hoc correction to the reconstruction of all data as will be described further in section 3.1.

\subsubsection{Short Orbit Spectrometer (SOS)}

The SOS is a magnetic spectrometer consisting of three resistive magnets in a $\mathrm{QD} \bar{D}$ configuration. Analogous to the HMS, the quadrupole magnet is used for focusing and the $18^{\circ}$ vertical bend dipole magnet combination is used for momentum dispersion. The spectrometer optics are operated in a point-to-point optical tune. The SOS acceptance yields a solid angle of $\approx 7 \mathrm{msr}$, which is defined by an octagonal collimator as in the case of the HMS. While the distance from the pivot to the detectors is rather short $(\approx 10 \mathrm{~m})$, the SOS momentum acceptance is relatively large $( \pm 20 \%)$. The SOS design allows for the spectrometer to be raised up to $20^{\circ}$ out of plane, but this feature was not used during $\mathrm{F}_{\pi^{-}}-2$. It should be noted that the optical axis of the SOS was measured to be $2.6 \mathrm{mrad}\left(0.15^{\circ}\right)$ below the horizontal, which has been taken into account in the data analysis.

The setting procedure for the SOS magnets is of great importance for determining momenta and reconstructed quantities. To ensure the SOS magnets always lie on the same part of the hysteresis curve a particular cycling procedure was used. This method entails the full degaussing of the magnets by ramping them to the maximum current value at a given polarity, re-zeroing the current value, cycling the 


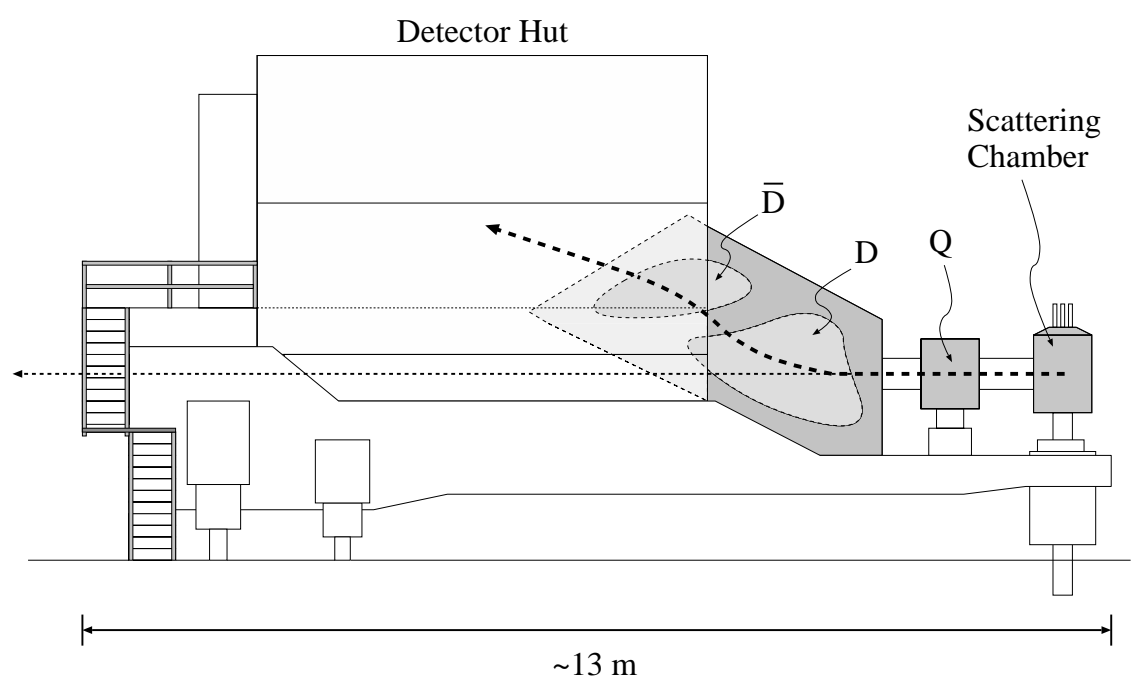

Figure 2.11: Schematic sideview of the SOS. The distance from the target to the $S O S$ focal plane is $\approx 10 \mathrm{~m}$. Figure from reference [92]

magnet to an empirically determined setpoint at opposite polarity, re-zeroing the magnet current and finally setting the magnets to the calculated set value.

Further complications on the measured particle trajectories arise due to the resistive nature of the SOS magnets. In regions of high fields corresponding to high momenta, saturation effects lead to a decrease of effective field length, and result in significant deviation of the true spectrometer central momentum value from the set value. A correction to the central momentum was parametrized based on elastic scattering data from hydrogen. Above central momenta of $1 \mathrm{GeV} / \mathrm{c}$ the effect can be as large as $1 \%$ at the maximum momentum of $1.7 \mathrm{GeV} / \mathrm{c}[55]$. A secondary saturation effect is the dependence of the spectrometer optics on reconstructed fractional momentum, $\delta$. This effect was first observed in $\mathrm{F}_{\pi^{-}}-1$ and was addressed with a momentum dependent correction to $\delta$ as described in detail in chapter 4.6 in reference [56]. It should be noted that the spectrometer optics dependence on $\delta$ was addressed 
in $\mathrm{F}_{\pi}-2$ by re-fitting of the momentum dependent spectrometer optics matrix.

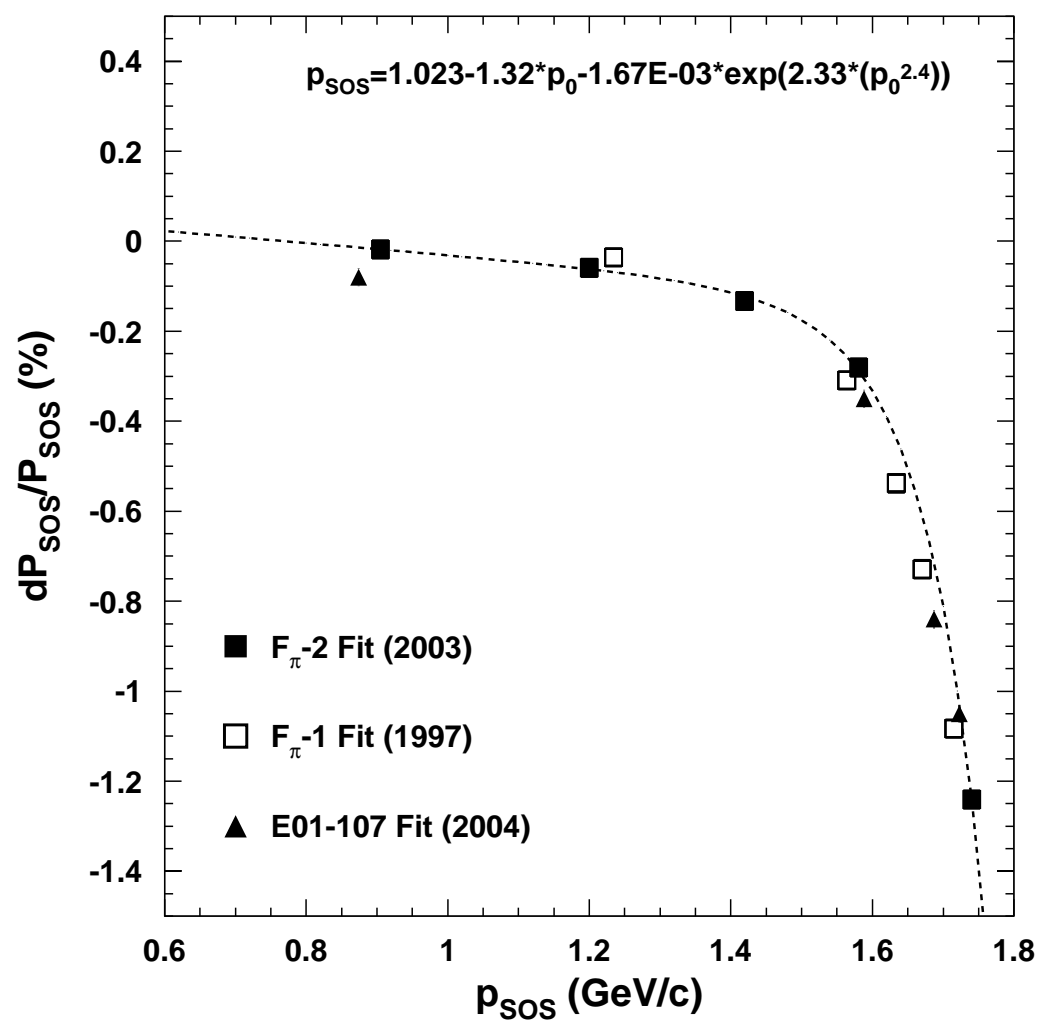

Figure 2.12: SOS central momentum Saturation correction. The SOS central momentum offset found for $F_{\pi}-1$ result in a true value for the central momentum about $0.45 \%$ higher than the nominal set point. This offset has been removed in the figure in order to compare the $F_{\pi}-1$ data points to the ones from $F_{\pi}-2$. The 2004 data points are from reference[ry]].

The angular acceptance limits of the SOS are defined by a HEAVYMET (2.5 inch $(3.75 \mathrm{~cm})$ thick) octagonal collimator analogous to the HMS. During F $\pi-2$ the large collimator was used in addition to the sieve slit, which was used for optics calibration. 


\subsection{Detectors}

The detector packages in the HMS and SOS are very similar. They consist of two horizontal drift chambers for track reconstruction, four scintillating hodoscope arrays used for triggering and time-of-flight measurements, and threshold gas Čerenkov detectors and lead-glass calorimeters for particle identification. The HMS detector package also includes an aerogel Čerenkov detector used for proton rejection. Pions and electrons are separated using a gas Čerenkov detector. The detector stacks, shown for the HMS in Figure 2.9, are located inside the respective spectrometer huts. The drift chambers (DC1 and DC2) are situated immediately after the dipole magnets at the front of the hut followed by two pairs of $\mathrm{x}-\mathrm{y}$ oriented scintillator hodoscopes enclosing the gas Cerenkov. The last detector is the lead glass calorimeter positioned at the back of the hut. The HMS aerogel Cerenkov detector is positioned between the first pair of $\mathrm{x}-\mathrm{y}$ scintillators and the gas Cerenkov. A particular difference between the spectrometers is that the $\mathrm{x}$ (vertical) oriented scintillator hodoscopes precede the $\mathrm{y}$ (horizontal) scintillators in the HMS while the order is reversed in the SOS. The individual detector components and their significance for data analysis are described in the following sections. A complete review of the detector packages including detailed geometry and performance evaluation can be found in reference [78]. 


\section{Drift Chambers}

Tracking drift chambers provide information on the position and trajectory of a particle. The basic operating principle is based on charged particle induced ionization of inert gases. The assembly consists of a chamber filled with a particular inert gas mixture, and an array of field and sense wires are located at well known postions inside the chamber. As a charged particle traverses the chamber, the gas mixture is ionized. Due to the potential difference created between sense and field wires, the ionized electrons are accelerated towards the sense wire closest to the original trajectory. The drift time to the collecting sense wires is measured using Time to Digital Converters (TDCs). The combination of the knowledge of the absolute position of the sense wires and the drift time allows then for the determination of the distance of the track of a charged particle from a particular collecting wire.

The HMS is equipped with a pair of drift chambers each consisting of six planes. The $x$ and $x^{\prime}$ planes determine the dispersive coordinates of the particle trajectory, while two $y$ and $y^{\prime}$ planes determine the nondispersive track position. The wires in the subsequent $u$ and $v$ planes are rotated $\pm 15^{\circ}$ with respect to the $\mathrm{y}$ coordinate as determined by the $x$ and $x^{\prime}$ planes. Each plane consists of a rectangular grid of sense wires separated by $\approx 1 \mathrm{~cm}$ and surrounded by "field wires". To ensure equipotential surfaces around each sense wire, the field wires are maintained at large negative high voltages $(\approx 1800-2500 \mathrm{~V})$. The particular voltage of each field wire depends on the distance of the field wire from the closest sense wire. The ionizing medium in the HMS drift chambers is an equal parts (1:1 by weight) mixture of 
Argon-Ethane, which is controlled by a gas handling system located on the outside of the experimental hall.

The combination of hit positions at each plane allows for the construction of the position and orientation of the particle trajectory at the focal plane. In order to resolve the ambiguity in the track reconstruction (left-right ambiguity) in the case of multiple hits the following criteria are implemented: if two similar planes measure the same coordinate, a "small angle approximation" is made and the particle is assumed to have passed between the two wires that fired. In the case where only one plane fired, all left-right combinations are considered and the track with the minimum $\chi^{2}$ is selected. The same method is used for unmatched planes like $u$ and $v$.

Particle tracking is done in the SOS with drift chambers similar in design to the HMS drift chambers. The SOS is also equipped with a pair of drift chambers consisting of six planes of wires. The $x$ and $x^{\prime}$ planes determine the dispersive particle trajectory but unlike in the HMS the $u$ and $v$ planes measure the nondispersive direction. The $u$ and $u^{\prime}$ planes are rotated $60^{\circ}$ clockwise with respect to the y coordinate determined by the $x$ and $x^{\prime}$ planes while the $v$ and $v^{\prime}$ planes are rotated $60^{\circ}$ counterclockwise. As in the HMS, the matched planes are offset by $0.5 \mathrm{~cm}$ perpendicular to the sense wire direction to resolve the left-right ambiguity in the case of multiple hits in both planes. Analogous to the HMS drift chambers a gas mixture of Argon-Ethane is used. 


\subsubsection{Hodoscopes}

Both HMS and SOS are equipped with four planes of scintillator hodoscopes divided into pairs of $\mathrm{x}-\mathrm{y}$ planes. Each pair contains one plane segmented in the dispersive direction (x-planes) and one plane segmented in the nondispersive direction (y-planes). Each plane is composed of several elements, the "bars" or "paddles", made of long narrow strips of BC404 scintillator material with photomultiplier tubes (PMT) attached to both ends. The scintillator paddles are arranged in overlapping configuration to eliminate gaps between the elements.

The principle of scintillation detectors can be summarized as follows: charged particles travelling through the scintillator material ionize electrons in the medium. The ionized electrons interact with the scintillating material exciting molecules to higher energy levels. The excited molecules fall back to the ground state emitting light in spontaneous emission. The light emitted propagates through the material via total internal reflection and is detected by photomultiplier tubes on either end attached to a lucite light guide. It should be noted that some light emitted at an angle smaller than the critical angle will not be internally reflected, but will also reach the PMTs, since the scintillator elements are wrapped in layers of reflecting material. The scintillator elements are also wrapped in two layers of dark Tedlar to ensure light-tightness. The reflecting materials are aluminum foil for the HMS and aluminized mylar for the SOS scintillator elements. The HMS scintillator paddles are $1.0 \mathrm{~cm}$ thick and $8.0 \mathrm{~cm}$ wide while the dimensions in the SOS are $7.5 \mathrm{~cm} \times 1.0$ $\mathrm{cm}$ for the $\mathrm{x}$ planes and $7.5 \mathrm{~cm} \times 1.0 \mathrm{~cm}$ for the $\mathrm{y}$ planes. The length of the paddles 
depends on the spectrometer and the location and orientation in the detector hut. It should be noted that the scintillator paddles are shorter in the SOS resulting in a generally better timing resolution per plane than in the HMS due to reduced attentuation. However, the overall time-of-flight resolution remains similar because of smaller separation distance between the front and back pair of planes in the SOS.

The arrangement of the two pairs of planes is similar in both spectrometers. However the separation between the front and the back planes and the order of the four planes is different for HMS and SOS. In the HMS the first plane is segmented vertically ( $x$ planes) and the second plane segmented horizontally ( $y$ planes) with a separation of $220 \mathrm{~cm}$ between the front and back pair. The plane order is reversed in the SOS and the pair separation is $180 \mathrm{~cm}$.

The main purpose of the scintillator hodoscopes is to provide the raw trigger for the data acquisition system and to determine the particle velocity by measuring the time of flight between the front and back planes. The hodoscope signals are read out through a combination of Analog to Digital Converters (ADCs), discriminators and Time to Digital Converters (TDCs), and (trigger) logic modules. These signals form the scintillator trigger signals S1X, S1Y, S2X and S2Y which are discussed in more detail in section 3.5.1. It should be noted that the electronics use constant threshold discriminators, which will result in particle dependent timing due to differences in energy deposited and resulting pulse height. The timing information from each scintillator paddle is corrected for pulse height and timing offsets using a software calibration routine. The development and detailed description of this routine can be found in [78]. 


\subsection{2 Čerenkov Detectors}

Both spectrometers are equipped with threshold gas Čerenkov detectors used for electron-pion separation. Charged particle separation with Čerenkov detectors utilizes the particle dependent threshold of Čerenkov radiation when particles pass through a given material. Čerenkov radiation is emitted when a charged particle traverses a material of index of refraction $n$ with velocity $\beta$ faster than the speed of light in the medium $(c / n)$. The angle, $\cos \theta_{c}=1 / n \beta$, at which the light is emitted depends on the velocity of the particle and is relatively small for fast particles and $n$ close to unity. The Čerenkov light is reflected from parabolic mirrors in the detector and focussed onto photomultiplier tubes.

The threshold property of Čerenkov radiation makes it possible to tune the medium in the detector to allow for specific conditions like electron and pion selection and their separation over a particular momentum range. Although the separation of electrons and pions is highly efficient, pion rejection is complicated by the presence of secondary (knock-on) electrons. These secondary electrons or $\delta$ rays are produced when a pion interacts with the material in front of the Čerenkov gas volume and can subsequently result in a hit in the Cerenkov detector. The mis-identification of pions due to $\delta$ rays was not a significant effect in this experiment.

The HMS Čerenkov detector is a cylindrical tank holding two mirrors and two photomultiplier tubes. The detector design allows for gas pressures in the tank above and below atmospheric pressure or at atmospheric pressure. Therefore, the use of the detector is not limited to $\pi / e$ separation at atmospheric pressure or below, but it 
can also be used to separate pions from protons using Freon-12 at super-atmospheric pressures. During $\mathrm{F}_{\pi}-2$ the HMS Čerenkov was filled with $C_{4} F_{10}$ gas at $0.47 \mathrm{~atm}$. The index of refraction at this pressure is 1.00066 giving an electron threshold of 7 $\mathrm{MeV} / \mathrm{c}$ and a pion threshold of $3.8 \mathrm{GeV} / \mathrm{c}$.

The SOS Cerenkov design is similar to the HMS Čerenkov but contains four mirrors and four phototubes. The Čerenkov medium was Freon-12 $\left(\mathrm{CCl}_{2} \mathrm{~F}_{2}\right)$ at a pressure of one atmosphere. At 1 atm, the refractive index of Freon-12 is 1.00108 which results in an electron threshold of $11 \mathrm{MeV} / \mathrm{c}$ and a pion threshold of $3 \mathrm{GeV} / \mathrm{c}$. This exceeds the SOS maximum central momentum by a factor of 2 .

\subsubsection{Lead Glass Calorimeter}

The lead glass calorimeter provides an additional means of selecting and separating electrons and pions. The lead glass calorimeter is positioned at the back of the detector hut for both spectrometers. Each lead glass calorimeter uses $10 \mathrm{~cm} \times$ $10 \mathrm{~cm} \times 70 \mathrm{~cm}$ blocks arranged in four planes and stacked 13 and 11 blocks high in HMS and SOS respectively. The entire detector is tilted by $5^{\circ}$ relative to the central ray of the spectrometer to minimize losses due to particles passing through the gaps between the blocks. To ensure light-tightness, each block is wrapped in aluminized mylar and Tedlar. The calorimeter signal from each block is read out by photomultiplier tubes attached at one side.

Particle detection using electromagnetic calorimeters is based on the production of electromagnetic showers in the lead glass material. Electrons entering the calorimeter interact with the fields of nuclei in the Pb-glass and radiate photons 
via the Bremsstrahlung process. The Bremsstrahlung photons produce electronpositron pairs that also radiate photons (either by Bremsstrahlung or Čerenkov radiation). The particular choice of thickness ensures that incident electrons or positrons deposit all their energy in the particle shower. The light radiated by the charged particles is collected by photomultiplier tubes on one end of the calorimeter and is proportional to the energy of the primary charged particle. Pions and muons entering the calorimeter do not produce a Bremsstrahlung shower and deposit a constant amount of energy $(\approx 300 \mathrm{MeV})$ in the calorimeter. However, pions can undergo nuclear interactions in the lead glass and produce particle showers similar to the electron and positron induced particle showers. The separation of electrons from other particles is based on the normalized energy deposited in all layers in the calorimeter. Pion contamination to the electron peak resulting from misidentified pions is a significant effect at momenta below $1 \mathrm{GeV}$ in the HMS. During $\mathrm{F}_{\pi}-2$ the SOS calorimeter was used in combination with the SOS Čerenkov detector to select electrons and to eliminate real $\pi^{+} / \pi^{-}$coincidence events. A representative calorimeter spectrum is shown in Figure 2.13 in the lower panel.

\subsubsection{HMS Aerogel Čerenkov Detector}

In addition to the standard particle identification detectors the HMS contains an aerogel threshold Čerenkov providing adequate hadron identification at central momenta above $3 \mathrm{GeV}$. Separating pions and protons at such high momenta is not possible by a direct measurement of the particle velocity due to the decreased

resolution of the time-of-flight $\left(\Delta \mathrm{t} \approx 1 / \mathrm{P}^{2}\right)$. Analogous to the gas Čerenkov detector, 

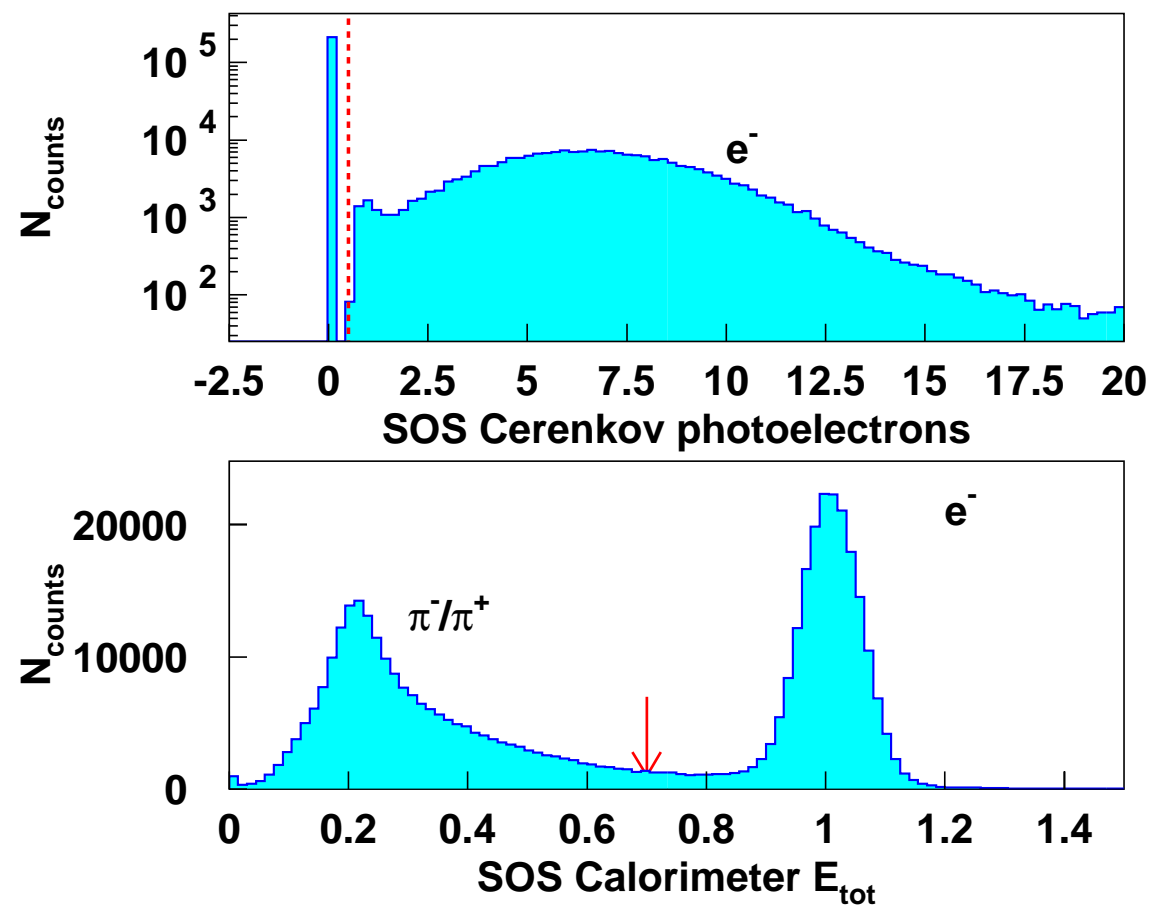

Figure 2.13: Representative SOS Čerenkov and SOS Calorimeter spectra. On average there are 7 photoelectrons per electron for the SOS Čerenkov with little position dependence for three of the four mirrors. Due to localized inefficiency in one of the mirrors (\#2), a low photoelectron cut $\left(N_{\text {photoelectron }}>0.5\right.$ was used. The calorimeter provides additional particle identification and eliminates residual real $\pi^{+} / \pi^{-}$coincidences. The arrow indicates the cut applied to select electrons.

the principle of particle detection with the aerogel Čerenkov is based on threshold Čerenkov radiation, which depends on the refractive index of the medium.

The medium in the HMS aerogel Čerenkov was chosen as to allow the detection of pions and separation from protons over a relatively wide momentum range (3.0-4.6 $\mathrm{GeV} / \mathrm{c})$. Aerogel is a hydrated silicon oxide of molecular structure $\mathrm{n}\left(\mathrm{SiO}_{2}\right)+2 \mathrm{n}\left(\mathrm{H}_{2}\right)$ and density ranging between 0.04 and $0.20 \mathrm{~g} / \mathrm{cm}^{3}$. The hydrate surrounding the molecule allows aerogel to assume an average refractive index between gases and liquids. For $\mathrm{F}_{\pi}-2$, aerogel with refractive index of $\mathrm{n}=1.030(\mathrm{n}=1.015$ was also available) was used, giving a pion threshold of $0.565 \mathrm{GeV} / \mathrm{c}$ and a proton threshold of 

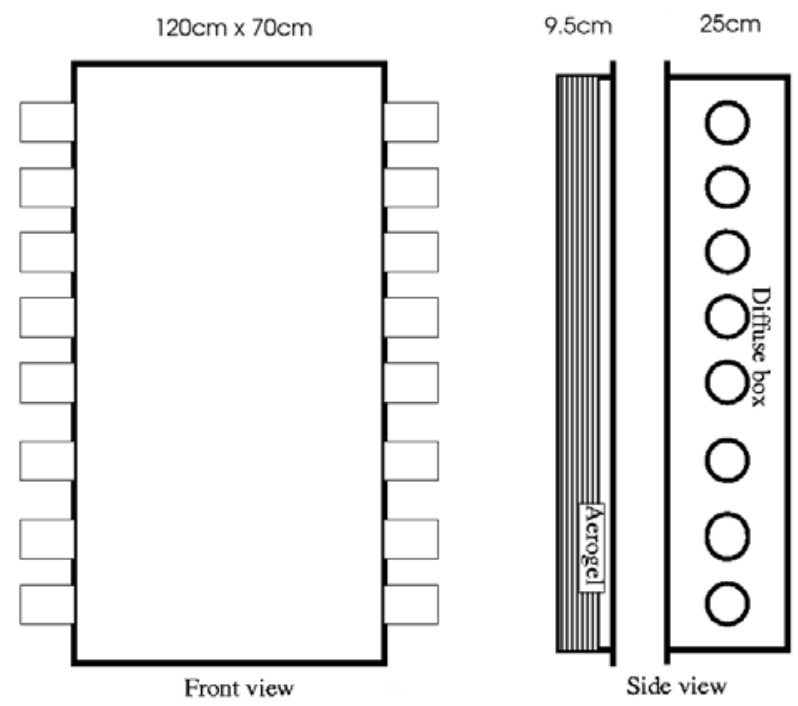

Figure 2.14: Schematic drawing of the HMS Aerogel Čerenkov detector. The detector was installed in the HMS in 2003 to provide more efficient particle identification at spectrometer central momenta above $3 \mathrm{GeV} / \mathrm{c}$. Two configurations with refractive indeces 1.03 and 1.015 are available. For $F_{\pi}$-2 aerogel with $n=1.03$ was used to reject protons.

$3.802 \mathrm{GeV} / \mathrm{c}$. The threshold momenta for muons and kaons are $0.428 \mathrm{GeV} / \mathrm{c}$ and $2.000 \mathrm{GeV} / \mathrm{c}$ respectively. The highest HMS momentum setting during $\mathrm{F}_{\pi}-2$ was $3.336 \mathrm{GeV} / \mathrm{c}$ so that proton/pion separation could be done adequately.

The aerogel Čerenkov detector consists of 650 tiles $\left(110 \times 110 \times 10 \mathrm{~mm}^{3}\right)$ arranged into nine $5 \mathrm{~mm}$ honeycomb sheets stacked in a $117 \times 67 \mathrm{~cm}^{2}$ tray. The individual layers were offset with respect to each other by $2-3 \mathrm{~cm}$ to minimize the loss of particles due to passing the detector without hitting any aerogel material. This also results in greater mechanical stability of the detector itself. A schematic of the aerogel detector is depicted in Figure 2.14. The Čerenkov radiation produced by particles traversing the aerogel material is collected by 16 ten-stage, 5 inch Photonis 


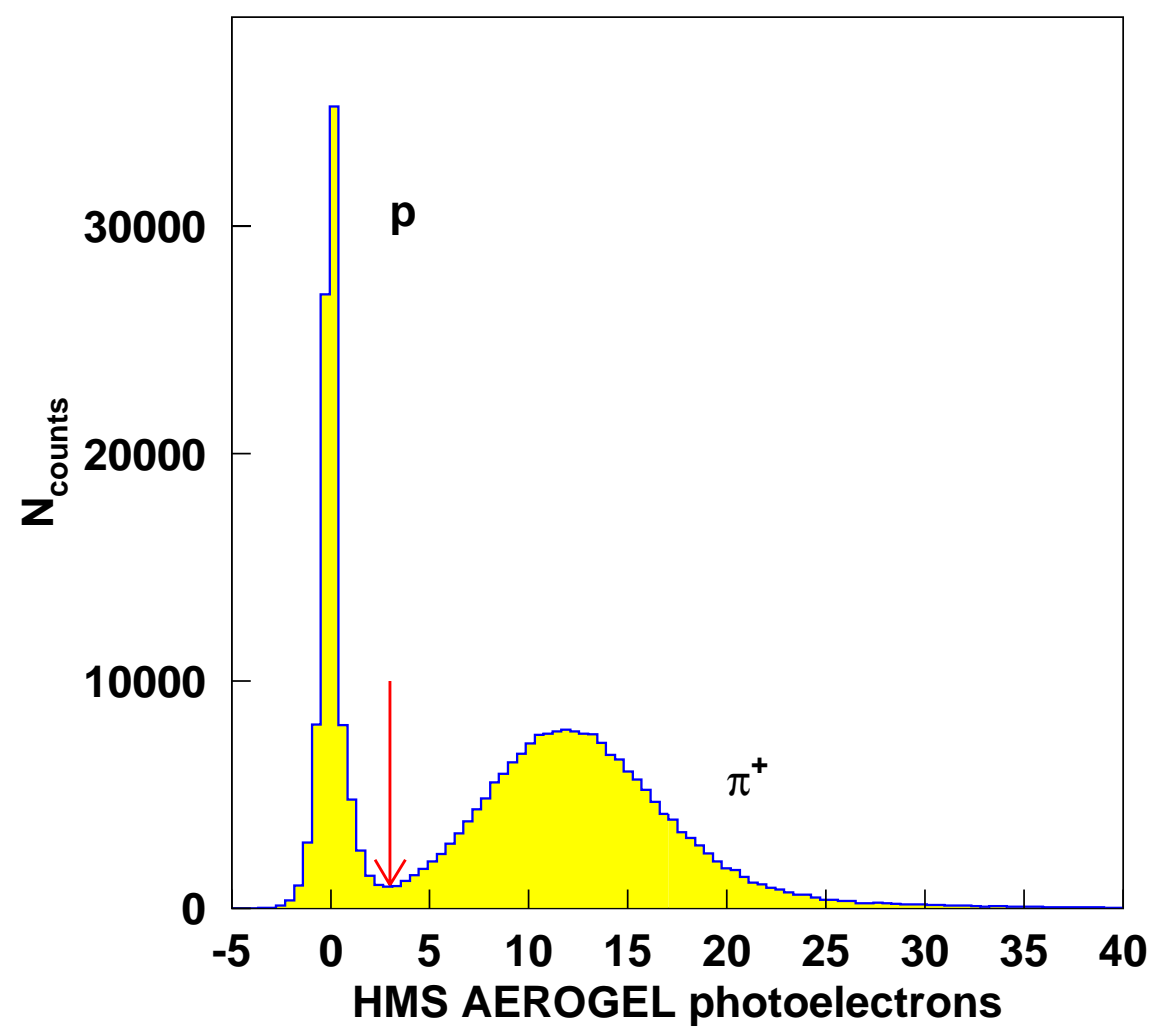

Figure 2.15: Aerogel Čerenkov Detector. The mean number of photoelectrons $\left(N_{p e}=\right.$ 12) is stable up to particle rates of $600 \mathrm{KHz}$. The arrow indicates the cut used in this analysis.

XP4572B photomultiplier tubes mounted on each side of the reflecting diffusion box. The reflective surface results in multiple reflection of the produced Cerenkov light before detection. The aerogel tiles were made light-tight by wrapping them in reflective material except for the surface facing the diffusion box with Millipore paper. To ensure high reflectivity from the internal walls, the inside of the diffusion box was covered with Membrane GSWP-0010 Millipore paper. The entire assembly of tiles was held in place by a $100 \mu \mathrm{m}$ stainless steel wire. Further detail on the design and testing of the aerogel Čerenkov detector can be found in [79]. 


\subsection{Trigger System and Data Acquisition}

The key part of the data acquisition is the trigger system designed to reduce the large rate of particles into a particular spectrometer by filtering real events from likely background events. In general, the goal of a hardware trigger system is to reduce electronic deadtime and total data volume while simultaneously keeping a high trigger efficiency. The Hall $\mathrm{C}$ trigger electronics consists of three components. The HMS and SOS form pretrigger or singles trigger signals based on their particular trigger configurations. These pretrigger signals are sent to the coincidence logic. If the pretrigger signals from both spectrometers occur within a particular time window a coincidence trigger is formed and the event is recorded by the data acquisition system.

\subsubsection{HMS Pretrigger}

The HMS trigger shown in Figure 2.16 is composed of signals from different HMS detectors. The main part in the formation of the HMS pretrigger is a signal from the scintillators (SCIN or STOF). The SCIN ("3/4") signal requires a signal from three out of four layers from the hodoscope scintillator planes within a timing window of $\approx 50 \mathrm{~ns}$. The advantage of requiring three out of four planes is a reduced dependence of the overall trigger efficiency on the efficiency of individual hodoscope paddles. Given the high efficiency of single hodoscope paddles the " $3 / 4$ " efficiency is generally quite high. In addition, the " $3 / 4$ " trigger can be used to determine hodoscope efficiencies and provides a way of monitoring the hodoscope performance 
during the experiment. The STOF is satisfied when two of the scintillator planes give a signal, with one of the signals coming from the front and the other from the back. This condition represents the minimum requirement of the scintillator information to determine the time of flight of a particle and is less restrictive than the SCIN condition. If SCIN is satisfied, STOF must also be satisfied.

If the scintillator signal is present, the pretrigger signal can be formed in one of two different configurations (ELLO and ELHI). The ELHHI part of the pretrigger (ELHI) is formed if all three of the following signals are present: The SCIN signal, the PRHI signal and the SHLO signal. PRHI and SHLO are signals from the calorimeter. PRHI is satisfied when the signal from the first layer of the calorimeter exceeds a particular "high" threshold while SHLO is formed when the total energy deposited in the calorimeter is above a particular "low" threshold. The ELLO pretrigger requires a two out of three coincidence of SCIN, PRLO and STOF. In addition, the ELLO signal requires the Cerenkov signal (CER). If CER is not present the ELLO signal will be vetoed. PRLO is a signal from the calorimeter and is satisfied when the energy deposited in the first layer of the calorimeter exceeds a particular low threshold.

Several pretrigger options are available at the PRETRIG module: 1) Standard electron trigger (ELREAL) 2) prescaled 3/4 pion trigger (PIPRE) 3) "open" (3/4) trigger (SCIN) to give an unbiased particle sample, and 4) pion trigger, 3/4 with Čerenkov veto, (PIONHI).

The OR of ELHI and ELLO forms the electron pretrigger or ELREAL signal. The advantage of using a two path electron pretrigger is a reduced sensitivity to 


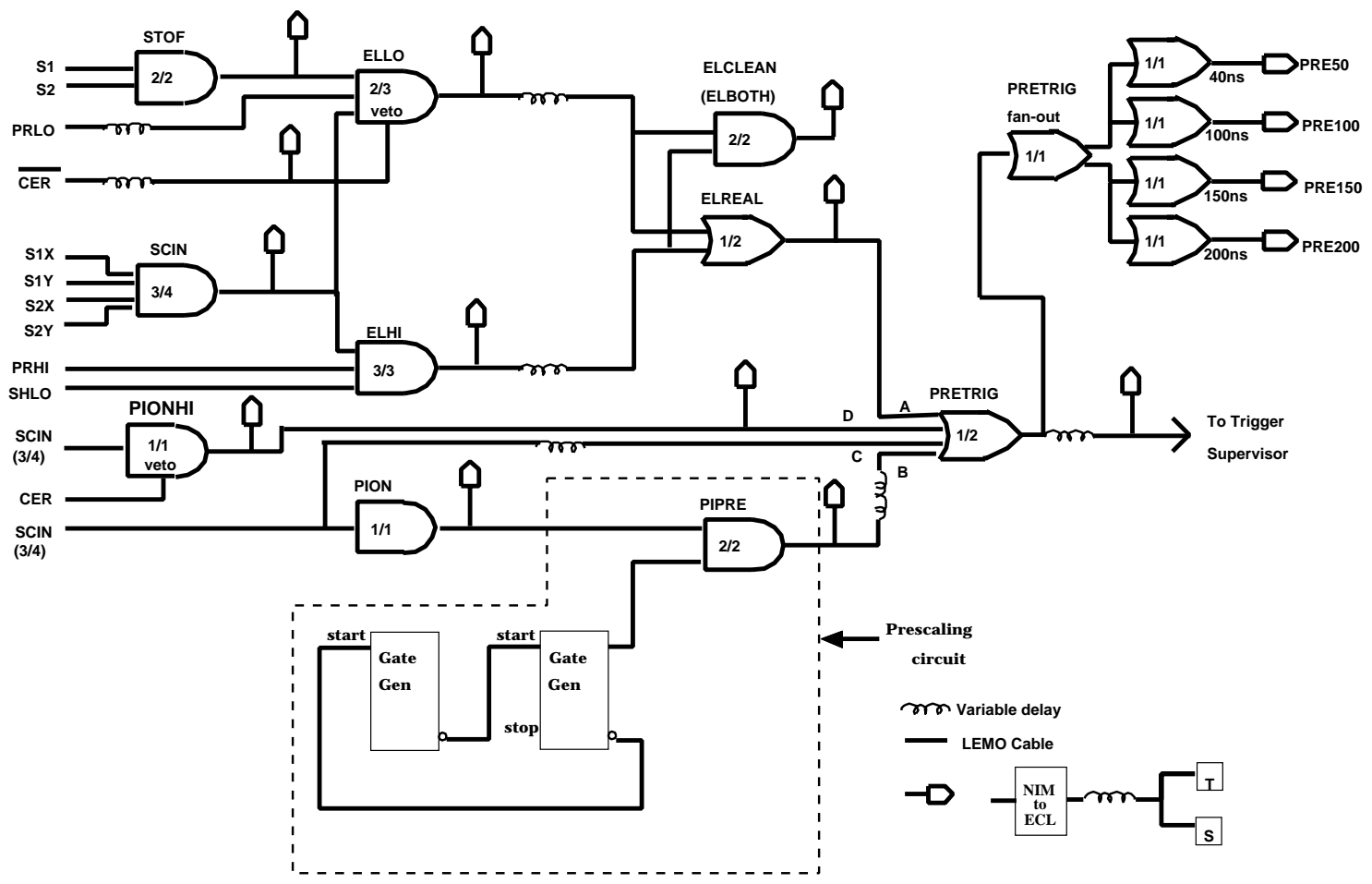

Figure 2.16: Schematic of spectrometer pretrigger logic. There are four options at the pretrigger module: 1) ELREAL standard electron trigger, 2) PIPRE prescaled $3 / 4$ pion trigger, 3) SCIN 3/4, and 4) PIONHI 3/4 with Cerenkov veto. Option 4 was added in the HMS trigger logic for $F_{\pi}$-2.

hardware problems in either the Čerenkov or calorimeter. Two copies of ELREAL are formed, of which one signal is sent to the HMS PRETRIG module where it is ORed with the prescaled pion signal, PIPRE. The PIPRE signal is formed to ensure that a sample of pions is recorded by the data acquisition system to allow for determining particle identification efficiencies like Čerenkov and calorimeter efficiencies. PIPRE is effectively a prescaled " $3 / 4$ " signal. The PRETRIG signal is also divided after the PRETRIG module into four signals (PRE50, PRE100, PRE150 and PRE200), with the width of each set according to its name (except for the PRE50 signal whose width is $40 \mathrm{~ns}$ ). These four signals are used in the ex- 
traction of the electronic deadtime discussed in section 3.5.3. In coincidence mode the HMS PRETRIG signal is sent to the coincidence electronics (8LM and trigger supervisor).

A fourth logic path was added during $\mathrm{F}_{\pi^{-}} 2$ as a HMS pion trigger (PIONHI). PIONHI is satisfied by the presence of the "3/4" SCIN signal in absence of CERHI, which is the HMS Čerenkov signal with a high threshold of $\mathrm{N}_{\text {photoelectrons }}$ $\approx 4$ in order to reject a large fraction of electrons. The PIONHI signal is sent to the HMS PRETRIG module and is also read out in the scaler TDCs. During the first part of the experiment the pion trigger condition was implemented as PIONHI ORed with the SCIN signal to allow for continous monitoring of the Čerenkov veto. The additional condition was determined to result in problems due to trigger blocking at high electron rates and reduced to PIONHI only for the rest of the experiment. Analysis of data taken with either trigger condition indicates no significant effect on the quality of the data.

\subsubsection{SOS Pretrigger}

The SOS pretrigger is formed in a similar way as in the HMS. Analogous to the HMS, ELLO signal is formed from the SCIN ("3/4"), STOF, PRLO given the presence of the SOS Čerenkov signal. The ELHI signal is formed if SCIN, PRHI and PRLO are present. The ELLO signal is then sent to the ELREAL module and two copies of the signal are sent to the SOS PRETRIG module. The PRETRIG signal is split into four copies after the PRETRIG module PRE50, PRE100, PRE150 and PRE200 for determining electronic dead time. Similar to 
the HMS trigger, the first part of the experiment required ELREAL ORed with PIPRE. This requirement was reduced to ELREAL at the same time the pion trigger condition was relaxed. Analysis of data taken with either trigger condition indicates no significant effect on the quality of the data.

\subsubsection{Coincidence Trigger}

Pretriggers from the HMS and SOS are sent to an 8LM LeCroy programmable logic unit (see Figure 2.17). The 8LM has six inputs and is used to separate events by type before they are forwarded to the Trigger Supervisor. The Trigger Supervisor (see Figure 2.17) effectively controls the readout of all detector ADC and TDC signals for an event.

The inputs to the 8LM logic unit includes inputs from HMS and SOS pretriggers as well as from the PED PRETRIG signal. PED PRETRIG is a random trigger that is only present for the first 1000 events of each run to determine the pedestals in the ADCs. The remaining three inputs on the 8LM are reserved for communication with the trigger supervisor. The Trigger Supervisor sends three signals to the $8 \mathrm{LM}$ indicating its status. The TS G0 signal is active whenever a run is in progress. In contrast, the TS EN1 signal is active only after the pedestal events have been taken in order to indicate the start of normal data taking conditions. The TS BUSY is active whenever the Trigger Supervisor is busy processing an event and cannot accept any other event.

The 8LM uses the three PRETRIG signals in combination with the information from the trigger supervisor to form eight outputs. Under normal data taking 


\section{Trigger Supervisor}

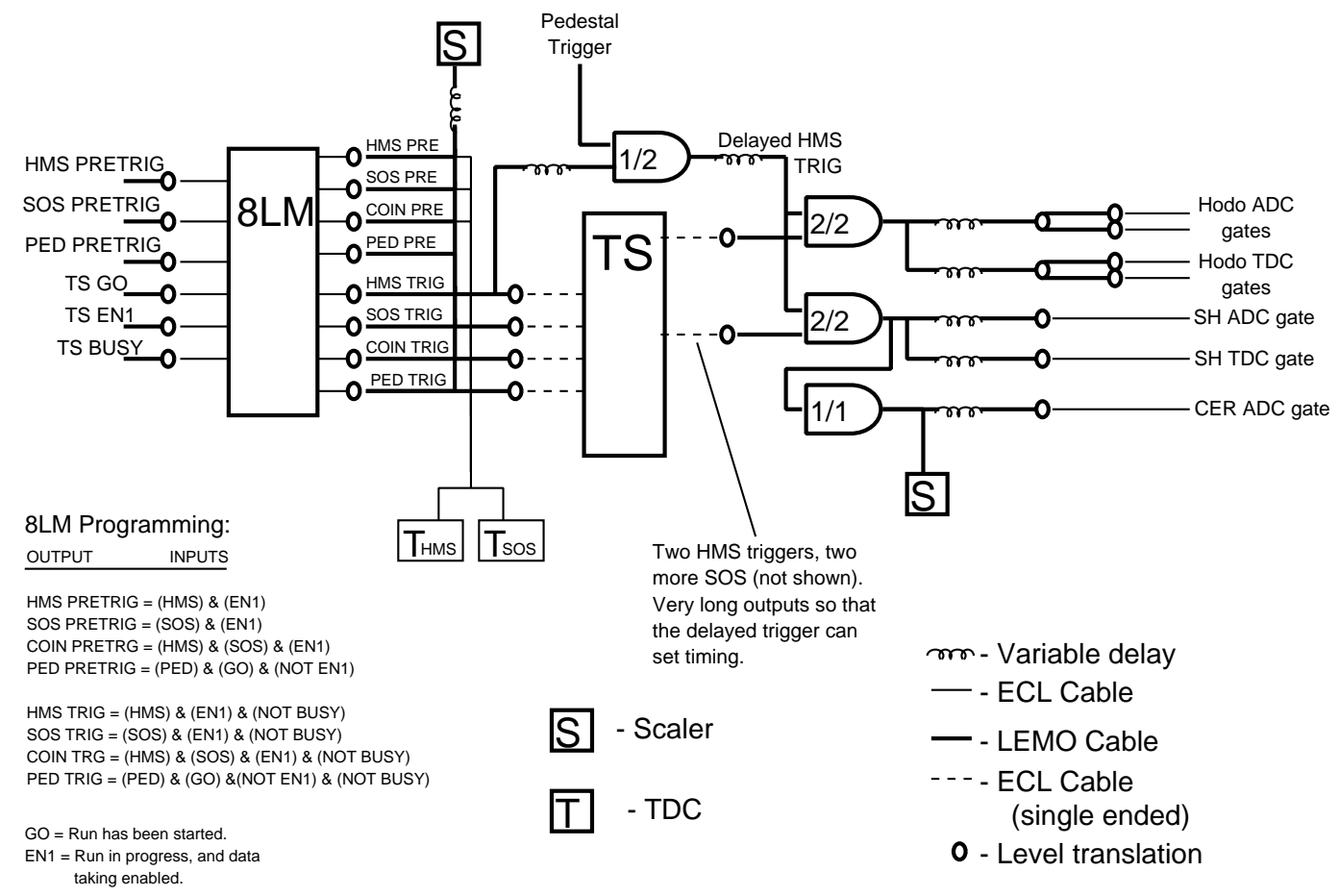

Figure 2.17: Schematic of spectrometer pretrigger logic. There are four options at the pretrigger module: 1) ELREAL standard electron trigger, 2) PIPRE prescaled $3 / 4$ pion trigger, 3) SCIN 3/4, and 4) PIONHI 3/4 with Cerenkov veto. Option 4 was added in the HMS trigger logic for $F_{\pi}$-2.

conditions the HMSPRE and SOSPRE signals are always present while the HMSTRIG and SOSTRIG signals are present during normal data taking only if the Trigger Supervisor is not busy. A coincidence pretrigger (COINPRE) is produced during normal data taking when the HMS PRETRIG and SOS PRETRIG signals arrive at the same time at the $8 \mathrm{LM}$. During $\mathrm{F}_{\pi}-2$, both the HMS PRETRIG and the SOS PRETRIG widths were $\approx 60$ ns resulting in an effective coincidence window of $\approx 120$ ns. All PRETRIG signals are always read out to scalers independent of the status of the Trigger Supervisor to allow for an estimation of the computer livetime. 
The TRIG signals are forwarded to the Trigger Supervisor. The Trigger Supervisor is programmed to prescale singles events that have only HMS or SOS TRIG signals with no associated COINTRIG signal. This effective removing of singles events is implemented to reduce the computer deadtime. When the Trigger Supervisor receives a COIN TRIG (either from HMS or SOS), it produces two long $(100 \mu \mathrm{s})$ pulses to the HMS or SOS readout electronics. These signals are ANDed with the delayed HMS or SOS TRIG signals to retime the signals with respect to the original HMS (SOS) trigger. The delayed signals are produced at the 8LM module and forwarded to enable the ADC readouts and TDC starts. This "retiming" is necessary to ensure that the timing of a particular spectrometer's readout is determined by the same spectrometer. The coincidence time is measured in two ways: one measurement is started by the delayed HMS TRIG signal stopped by the SOS TRIG and the other is started by the delayed SOS TRIG signal and stopped by the HMS.

\subsection{Data Acquisition}

The data acquisition was managed using CODA (CEBAF Online Data Acquisition) software [80]. For each run three types of events were recorded: detector information recorded by ADCs and TDCs, scaler information, and events from the EPICS database.

The readout electronics (ADCs, TDCs and scalers) were located in FASTBUS and VME crates. Each crate was managed by a separate ROC (Read Out Controller) CPU that were read directly by the CODA software through FDDI/ethernet net- 
work. Both ADCs and TDCs were read out once per event while the scalers were read out every two seconds. The EPICS database included information like beam current, raster, magnet settings, spectrometer angle and beam positions read out at intervals of 2 and/or 30 seconds. 


\section{Chapter 3}

\section{Data Analysis}

The raw data collected by the data acquisition system were processed by the standard Hall $\mathrm{C}$ analysis engine (ENGINE), which decodes the data into physical quantities on an event by event basis. In the following sections the main components of the data analysis such as tracking and event reconstruction are discussed along with calibration and optimization procedures of the detectors.

\subsection{Tracking and Reconstruction}

To determine the trajectory and momentum of a particle at the interaction vertex the coordinates of a valid track detected in the detector hut have to be transformed back to the target. Finding a valid track is done via the pair of drift chambers in each spectrometer. Each chamber has six planes of wires and a signal of at least five planes is required by the tracking algorithm to start constructing a track for a given event. Given the required minimum number of "hits" the aim of the tracking algorithm is to determine the parameters of the straight line that results in the best fit to the drift chamber coordinates. The hit coordinates are determined from a comparison of the hit position and the location of hit wire. All distances are calculated from the drift time measured. These coordinates determine the so called "space points" if their distance does not exceed a particular slop value. The combination of all space points in a chamber form the so called "stubs", a small part of the final track. To find the final track the tracking algorithm performs a $\chi^{2}$ minimization fitting a straight line through both chambers with the constraint that 
the resulting track has to be consistent with the stubs in each chamber. In the case of multiple tracks, the track with a hit in the calorimeter and closest to a paddle that registered a hit in the second scintillator plane is selected. The complete hierarchy of selection criteria is described in more detail in [81]. Projecting the fitted track to the detector focal plane situated halfway between the two drift chambers yields the position $\left(x_{f p}, y_{f p}\right)$ and trajectory $\left(x_{f p}^{\prime}, y_{f p}^{\prime}\right)$ for the particle track.

The knowledge of the four spectrometer coordinates at the focal plane allows for the reconstruction of the corresponding target coordinates via a matrix transformation which can be expressed in terms of a power series expansion:

$$
x_{t a r}^{i}=\Sigma_{i, j, k, l}^{N} M_{j k l m}^{i}\left(x_{f p}\right)^{j}\left(y_{f p}\right)^{k}\left(x_{f p}^{\prime}\right)^{l}\left(y_{f p}^{\prime}\right)^{m}
$$

where $x_{t a r}^{i} \epsilon\left(x_{t a r}^{\prime}, y_{t a r}, y_{t a r}^{\prime}, \delta\right)$ and $M_{j k l m}^{i}$ denote the elements of the reconstruction matrix. In particular, $x_{t a r}^{\prime}$ is the out of plane scattering angle at the target, $y_{t a r}$ is the in-plane position of the event in spectrometer coordinates, $y_{t a r}^{\prime}$ is the in plane scattering angle relative to the spectrometer central angle and $\delta$ is the fractional momentum deviation with respect to the central momentum of the spectrometer. The sum over indices is constrained by

$$
j+k+l+m \leq N
$$

where $N$ is the order of the series expansion. In this analysis all matrix elements up to sixth order $(N=6)$ are included for both HMS and SOS. It should be noted 
that given the available constraints only four of the five target quantities can be reconstructed. By default it is assumed that the beam is centered at $X_{t a r}=0$ and any deviation from zero is obtained from the fast raster information. The leftright symmetry of the spectrometers restricts the allowed combinations of target quantities and not allowed combinations are called "forbidden". The forbidden combinations can be expressed in terms of the indices as described in [56]. However, forbidden matrix elements have nonzero values if the midplane symmetry of the spectrometer is broken. It is known [82] that the symmetry is indeed broken due to offsets in the focussing magnets in the spectrometer, and the entire hierarchy of matrix elements was included in subsequent analysis.

The matrix elements are optimized using an iterative fitting procedure which is based on a starting model from the COSY INFINITY program [83]. For the purpose of optimizing matrix elements a special set of data is required which is taken with a carbon optics target and sieve slit collimator described in section 2.7.1 to fit $x_{t a r}^{\prime}$, $y_{\text {tar }}$ and $y_{\text {tar }}^{\prime}$. Using the model reconstruction matrix as a starting point, the holes in the sieve slit collimator through which an event passed can be identified. Given the sieve hole coordinates the matrix is modified using an iterative optimization procedure $[84,85]$ so that the event is reconstructed to the location of the sieve holes. The $\delta$ matrix elements are generally fitted using elastic electron scattering from carbon or hydrogen.

A detailed description of the matrix element fitting procedure can be found in Chapter 4 in reference [56]. For the HMS, matrix elements based on a fit from $\mathrm{F}_{\pi^{-1}}$ were used. It was found in this analysis that the matrix elements are not 
well constrained for particles outside a vertical angular acceptance of $\pm 60 \mathrm{mrad}$. This results from the fact that the outermost holes of the sieve slit do not entirely cover the acceptance of the octagonal collimator. To extend the valid region of the out of plane matrix elements, optics data were taken in 2003 with a shifted sieve slit extending the vertical range of the outermost sieve hole columns by $\pm 2.7 \mathrm{~cm}$. The $x_{t a r}^{\prime}$ matrix elements were then optimized following the procedure outlined in [86]. In addition, $y_{\text {tar }}^{\prime}$ matrix elements were optimized using the same optics dataset to address $y_{\text {tar }}^{\prime}$ acceptance edge effects observed in elastic data. The sensitivity to the acceptance and the implication to the data analysis will be discussed further in section 5.5. For the SOS matrix elements a fit from optics data taken at $\mathrm{P}_{S O S}$ $\approx 1.4$ from 1997 were used. It should be noted that the fitting of the SOS matrix elements is complicated by saturation effects at SOS central momenta above $\mathrm{P}_{S O S} \approx$ $1.0(\mathrm{GeV} / \mathrm{c})$. In particular, matrix elements exhibit saturation behavior away from the momentum at which the matrix elements were fit. Saturation effects for SOS $\delta$ matrix were noticed in previous experiments $[55,56]$ especially at momenta $(>1.4$ $\mathrm{GeV} / \mathrm{c}$ ). To properly describe the momentum dependence of SOS optics, data were taken for particular, small delta regions in 2003 and new $\delta$ matrix elements were fit. It should be noted that in this analysis SOS matrix elements from the 1997 fit are used with a correction to reconstructed $\operatorname{SOS} \delta$ as described in section 4.6 in reference [56]. The angular and position resolutions were between 0.3 and $3.3 \mathrm{mrad}$ and 0.9 and $1.1 \mathrm{~mm}$ in the 1997 matrix element optimization. To improve the resolution in angle and position, correction functions in terms of polynomial expansions up to 10th order were fit for $x_{t a r}^{\prime}, y_{t a r}$ and $y_{t a r}^{\prime}$ matrix elements. The absolute size of this 
effect is on the order of $1 \mathrm{mrad}$ and therefore relatively small.

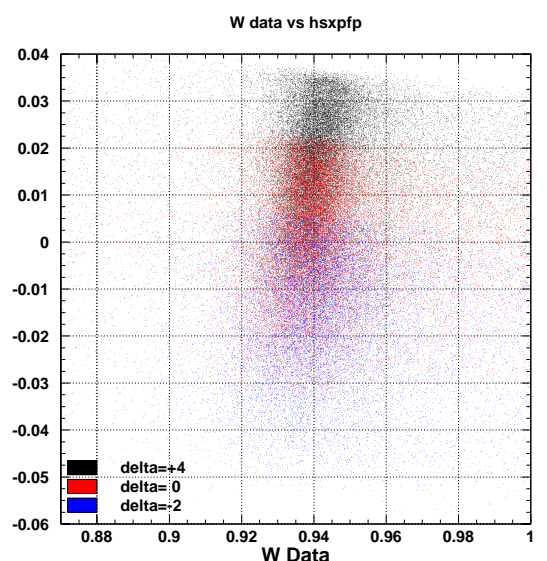

(a) No $x_{f p}^{\prime}$ correction.

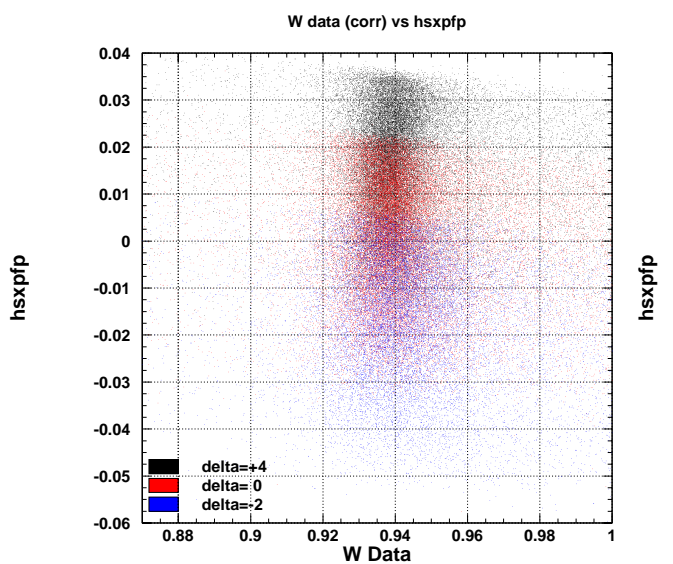

(b) $x_{f p}^{\prime}$ correction applied to reconstruction.

Figure 3.1: Reconstructed invariant mass, $W$, from an elastic (e, $\left.e^{\prime}\right)$ delta scan as a function of $H M S x_{f p}^{\prime}$ at $P_{H M S}=4.005 \mathrm{GeV} / \mathrm{c}$. If the matrix elements are correct, $W$ is expected to reconstruct to the proton mass independent of target and focal plane quantities. With no further correction, the centroid of the elastic peak varies by 2-3 MeV across the $x_{f p}^{\prime}$ acceptance. Applying an ad-hoc $x_{f p}^{\prime}$ correction to the reconstruction of the data eliminates most of the correlation.

If the matrix elements accurately describe the magnetic optics of the spectrometers, the reconstructed invariant mass in elastic electron singles data is expected to be independent of the focal plane and target variables. However, elastic electron singles data indicate that there exists such a correlation in HMS $x_{f p}^{\prime}$ (see Figure 3.1). This effect has been observed previously [55] and was corrected by fitting a momentum dependent linear correction to HMS delta:

$$
\delta^{\prime}=\delta+\epsilon_{\text {corr }} \times h \operatorname{sxpfp}
$$

where the correction factor $\epsilon_{\text {corr }}$ is fit over a range of $\mathrm{P}_{H M S}$ values and parametrized 


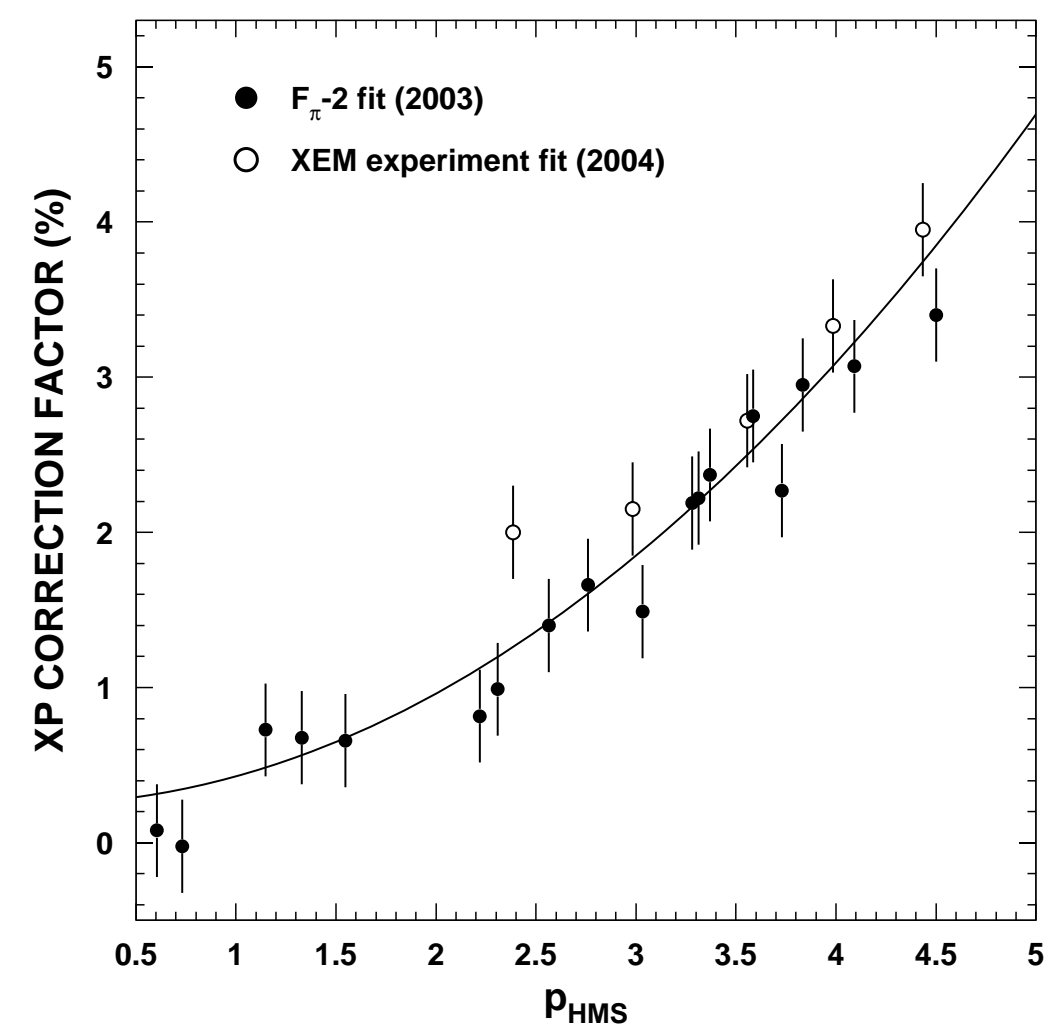

Figure 3.2: HMS $\left(\delta, x_{f p}^{\prime}\right)$ correction $\left(\epsilon_{\text {corr }}\right)$ as a function of HMS central momentum. This correction is applied to all data in the $F_{\pi}$-2 analysis. The 2004 data were obtained from reference [71].

by a continous function to describe the momentum dependence of the correction. Using an equivalent fitting procedure a correction was fitted based on data from 2003 and 2004 up to $\mathrm{p}_{H M S}=4.7(\mathrm{GeV} / \mathrm{c})$. Although most of the correlation is eliminated by this ad-hoc correction, higher order effects remain and may be apparent in the shape of the correction especially at lower HMS central momenta. A likely origin of the observed optics effect is a missetting of the third quadrupole. This hypothesis is supported by the fact that the optics effect can be modeled in the standard Hall C Monte Carlo. Details of the simulation of the effect can be found in section 4.3 
of reference [55].

\subsection{Spectrometer Optics}

The standard Hall C magnetic spectrometers, HMS and SOS, were used for particle detection in $\mathrm{F}_{\pi}-2$. To discuss the optical properties of these spectrometers two coordinate systems have to be defined. The lab frame and spectrometer frame coordinate systems are defined as follows: in the lab frame the $\mathrm{z}$ direction is defined along the beam direction with the $\mathrm{y}$-axis perpendicular so that $\mathrm{y}>0$ is pointing upwards and hence the x-axis pointing to the left. In contrast, the z-axis in the optical transport coordinate system always points towards the spectrometer with the $\mathrm{x}$-axis vertical so that $\mathrm{x}>0$ is pointing downwards with the $\mathrm{y}$-axis completing the right handed coordinate system.

The HMS acceptance is defined by the HMS octagonal collimator and ideally the validity of the HMS reconstruction matrix precisely matches this region. However, as mentioned earlier, the HMS sieve slit collimator does not entirely cover the octagonal collimator (see Figure 3.3). Since the reconstruction matrix elements are fitted with the sieve slit collimator there are regions at the edges of the acceptance for which fitted matrix elements do not provide sufficient guidance on the reconstruction.

To extend the region of validity of the recontruction matrix to the acceptance of the octagonal collimator, sieve slit data were taken in 2003 with a shifted sieve collimator. The HMS sieve slit collimator consists of arrays of sieve holes arranged in nine horizontal rows separated by $1.524 \mathrm{~cm}$ and seven vertical columns separated 


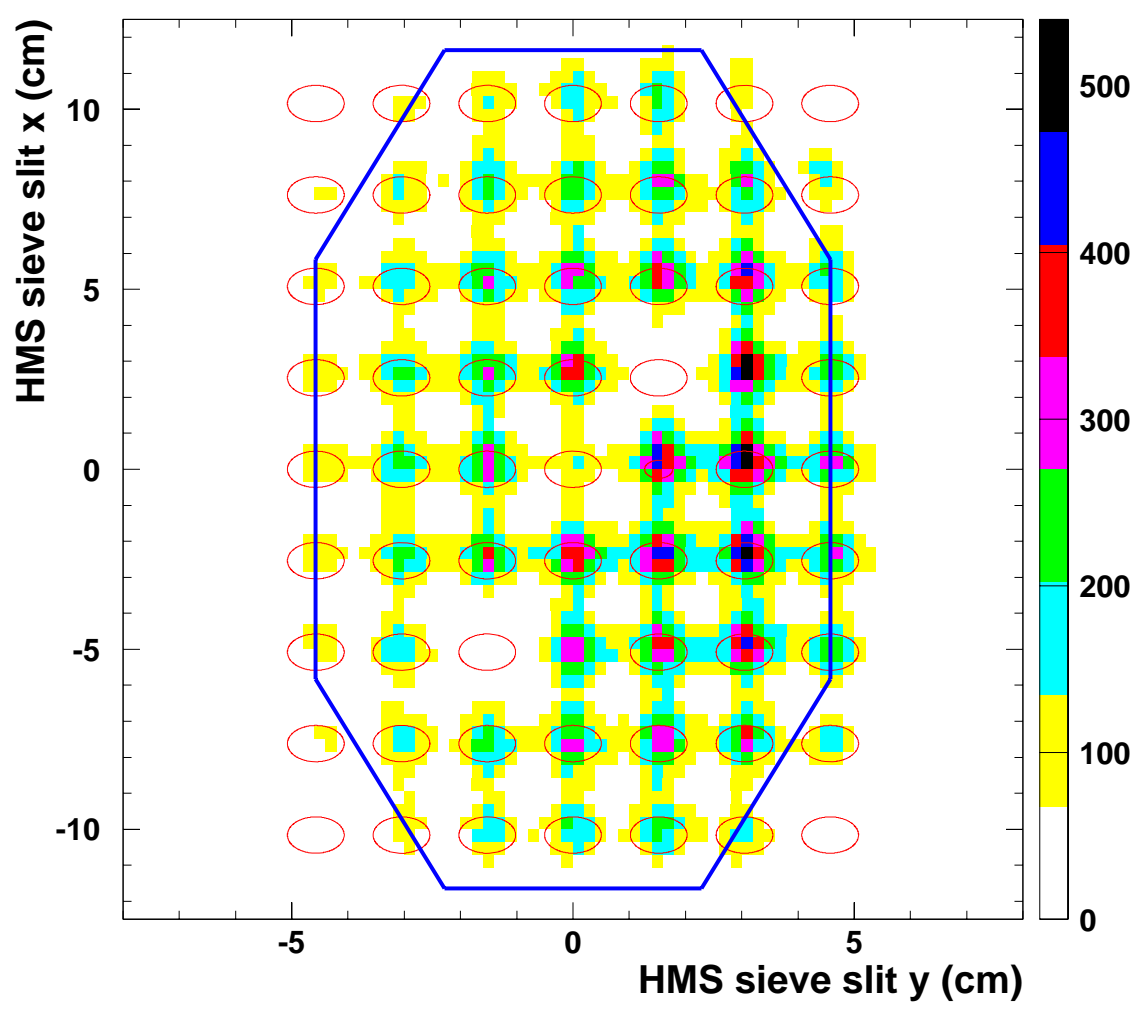

Figure 3.3: Reconstruction of the HMS sieve hole pattern. The solid line denotes the acceptance defined by the octagonal collimator. Note that the sieve holes in the figure are denoted by ellipses, while the collimator sieve holes are circular.

by $2.540 \mathrm{~cm}$. Each sieve hole has a diameter of $0.508 \mathrm{~cm}$ except for the central sieve hole whose diameter is $0.254 \mathrm{~cm}$. The outermost vertical sieve holes are located at $\pm 10.16 \mathrm{~cm}$, which corresponds to \pm 60.5 mrad. By shifting the sieve collimator by $1 / 2$ row $(1.27 \mathrm{~cm})$ on top and bottom the vertical acceptance was extended to \pm 68 mrad. The data were obtained with a "quintar", a special thin carbon target assembly used for optics calibrations. The assembly consists of five target foils defining five vertices along the beam axis. The reconstruction of the five quintar targets and the reconstruction of the sieve slit for both shifted and unshifted sieve 
collimator are shown in Figure 3.4. The distance from the center of the target to the center of the sieve collimator is $167.6 \mathrm{~cm}$.
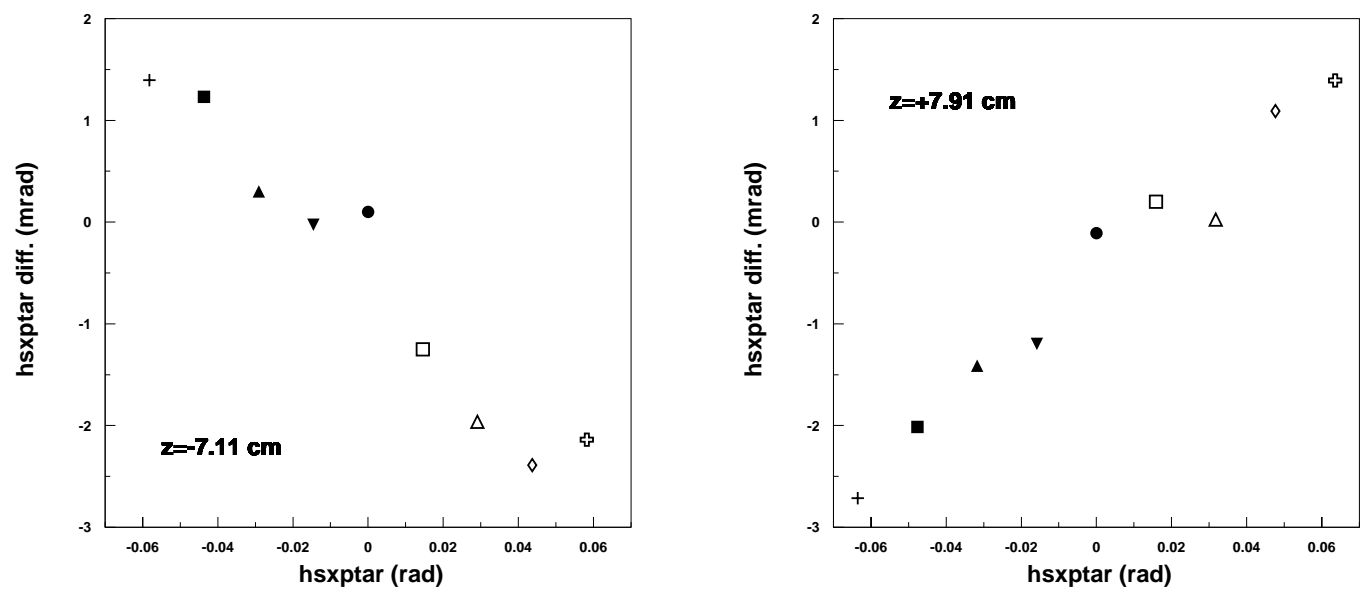

Figure 3.4: Difference between the reconstructed out of plane and the value calculated from sieve hole location and geometry, not corrected for horizontal beam position, for the outermost quintar targets at $z=-7.11,+7.91 \mathrm{~cm}$. The symbols represent the 9 vertical columns of the sieve collimator. The difference between reconstructed and calculated $x_{\text {tar }}^{\prime}$ varies by 4.5 mrad across the acceptance. This slope may be attributed to a modification in the out-of-plane angle, $x_{t a r}^{\prime}$, resulting in a nonzero vertical beam position in the optical plane of the spectrometer, which in turn affects the reconstuction of $x_{\text {tar }}^{\prime}$. The sign of the slope depends on the target position along the beam, since $x_{t a r}^{\prime}=\frac{d x}{d z}$.

The HMS matrix elements in $x^{\prime}$ and $y^{\prime}$ were optimized from the data set taken with the shifted sieve slit collimator and using the fitting routine described in section 3.1. $x^{\prime}$ and $y^{\prime}$ matrix elements are optimized via the reconstructed target quantities from the measured focal plane quantities on an event by event basis. The data used for the optimization were taken at $P_{H M S}=3.5(\mathrm{GeV} / \mathrm{c})$ and $\theta_{H M S}=15^{\circ}$ and $P_{H M S}=1.7(\mathrm{GeV} / \mathrm{c})$ and $\theta_{H M S}=18^{\circ}$ respectively. The second data set was taken before the $\mathrm{F}_{\pi}-2$ experiment in 2003. The optimized coefficients were determined from a data sample defined by cuts on acceptance $(|\delta|<10 \%)$, HMS Čerenkov and 
$y_{\text {tar }}$. Events passing these cuts and clearing a sieve hole are kept for fitting the new matrix elements. For approximate equal weighting of each sieve hole an additional cut was placed on the number of events (limiting 100 events per sieve hole). Both the beam position with respect to the target center and the dependence of the distance to the sieve slit on target position along the beam were also taken into account in the fit. The optimization of the in-plane angle was performed in a similar fashion using data from the same measurement. Figure 3.4 shows the difference between predicted and reconstructed out-of-plane and in-plane angles after the optimization. The prediction for each angle was calculated using the known location of the sieve holes and the distance of the target center with respect to the sieve slit.

\subsubsection{Spectrometer Optics for Extended Targets in $y_{t a r}$}

The spectrometer reconstruction does not provide information on the position coordinate at the target, $x_{t a r}$, and it is assumed that the beam is centered at zero. This approximation is generally valid for short targets with small extent along the $\mathrm{y}$-axis $\left(\mathrm{y}_{\text {tar }}\right)$ in the spectrometer coordinate system. The relation for the target length between $y_{t a r}$ and beam coordinates is given by,

$$
y_{\text {tar }}=-x \cos (\theta)+z \sin (\theta),
$$

where $x$ is the horizontal beam position, $z$ is the position of the target along the beam and $\theta$ is the spectrometer central angle. Typical 4-cm cryotargets have a length of $<1 \mathrm{~cm}$ along $y_{\text {tar }}$ for HMS central angles up to $30^{\circ}$. However, for extended 
targets like the carbon quintar target, foils are located at $y_{t a r}=2.2-2.4 \mathrm{~cm}$ even at forward angles. The angle for events from these positions results in a vertical beam position, $x_{t a r}$, different from zero in the $z=0$ plane (ie. optical plane of the HMS), as illustrated in Figure 3.5, which in turn affects the reconstructed quantities as described below.

The correct vertical beam position is determined assuming that the central hole of the sieve slit is centered on the HMS optical axis. When the beam is vertically not centered in the optical plane, $\left(x_{t a r} \neq 0\right)$, the reconstructed quantity, $x_{t a r}^{\prime}$, corresponding to the central hole of the sieve slit collimator is different from the expected value, $x_{t a r}^{\prime}=x_{0} / D$, where $x_{0}$ is the measured value at the target and $D$ is the distance from the target to the sieve slit collimator. The difference in the reconstruction results from a modification of the focal plane quantities (due to the beam offset at the target), which are used to reconstruct $\delta, x_{t a r}^{\prime}$, but not $x_{t a r}$. The size of the effect can be calculated to first order from the forward and backward optics matrices. Taking $\delta=0$ corresponding to the middle of the focal plane, the effect of a vertical beam offset on reconstructed $\delta$ and $x_{t a r}^{\prime}$ can be expressed,

$$
\begin{aligned}
& x_{\text {corr }}^{\prime}=x_{\text {recon }}^{\prime}-1.14 x_{0} \\
& \delta_{\text {corr }}=\delta_{\text {recon }}-0.77 x_{0}
\end{aligned}
$$

where the subscripts corr and recon denote the true and reconstructed values of $\delta$ and $x_{t a r}^{\prime}$ and $x_{0}$ is the measured value at the target. The resulting momentum offset, $\delta$, is $-0.077 \% / \mathrm{mm}$ for the HMS and $-0.039 \% / \mathrm{mm}$ for the SOS. The $x_{t a r}^{\prime}$ 
offset for the trajectory through the central sieve hole is proportional to a vertical beam offset: $x_{t a r}^{\prime}=\frac{x_{t a r}}{-1.73 m r a d / m m}$ for the HMS and $x_{t a r}^{\prime}=\frac{x_{t a r}}{-0.35 \mathrm{mrad} / \mathrm{mm}}$ for the SOS. A more detailed discussion including the calculation for SOS reconstruction can be found in reference [87].

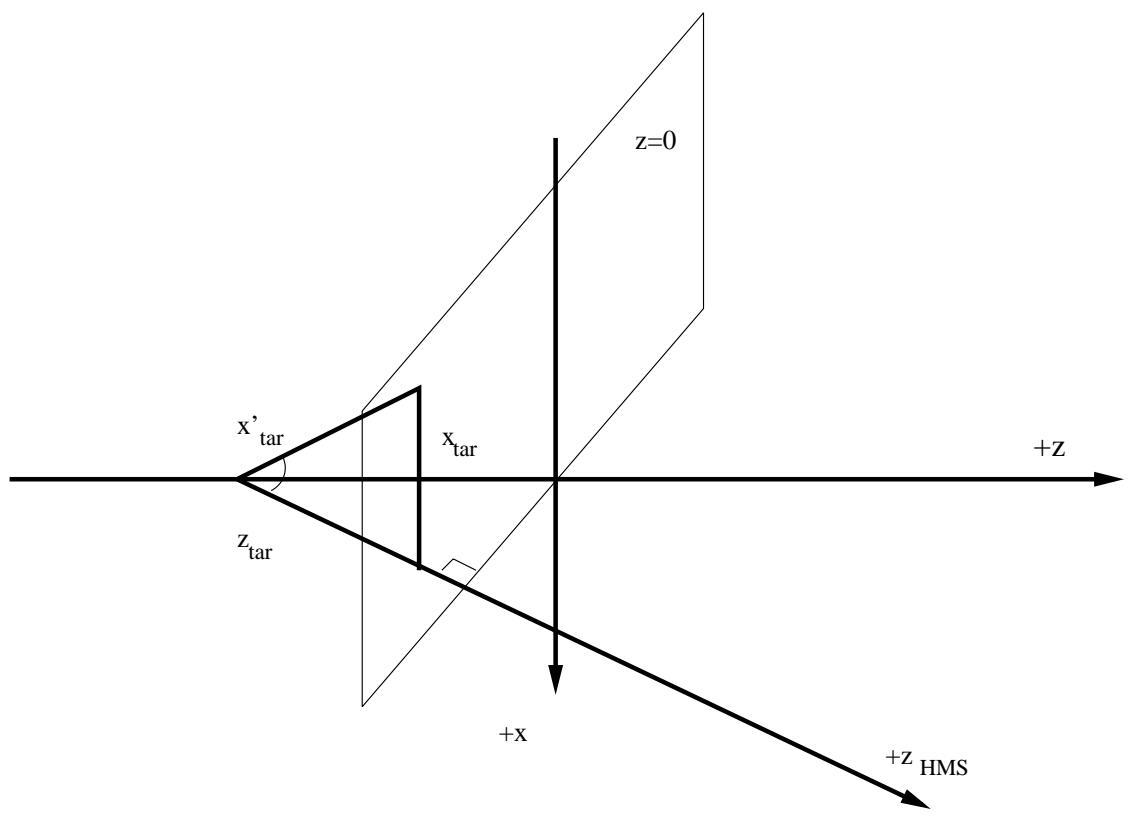

Figure 3.5: Diagram of the target in spectrometer coordinates. The angle is different at extreme target positions relative to $z=0$ resulting in a vertical beam position offset in the optical plane. Note that the sign of $x_{t a r}^{\prime}=-\frac{x_{t a r}}{z_{\text {tar }}}$ changes with the sign of $z_{\text {tar }}$, since the scattering angle is always defined to be positive.

At least two effects contribute to the modification of the reconstruction and can be observed in the focal plane. Figure 3.6 shows typical focal plane patterns for quintar targets located at $z=-7.11 \mathrm{~cm}$ and $z=+7.91 \mathrm{~cm}$. The nine vertical sieve hole columns are visible in both cases for a projection of $\mathrm{x}_{f p}$ and $\mathrm{x}_{f p}^{\prime}$ and a shrinking and expansion like behavior can be ovserved for these extreme negative and positive positions along the beam. The total variation in angle relative to $z=0$ is on the order of $7 \mathrm{mrad}$ for the outermost sieve holes and can be attributed to a combination of 
two effects. First, the distance to the sieve slit collimator is $\approx 4 \%$ larger for the outermost target foils relative to the central one. Using geometrical arguments the target length in the spectrometer system can be written,

$$
z_{\text {tar }}=y_{\text {tar }}\left(\sin (\theta)+\frac{\cos (\theta)}{\tan \left(\theta-y_{\text {tar }}^{\prime}\right)}\right)
$$

where $y_{t a r}$ is the position of the interaction vertex in the spectrometer coordinate system, $\theta$ is the spectrometer central angle and $y_{\text {tar }}^{\prime}$ is the cartesian in plane angle in the spectrometer coordinate system. The range of interaction verteces along the beam for the quintar target results in a change in reconstructed angle by 2.5 mrad. After taking into account the position of the quintar target foils along the beam, a discrepancy of $\approx 4.5 \mathrm{mrad}$ remains. This effect can be attributed to $\mathrm{x}_{\text {tar }} \neq 0$ in the optical plane.
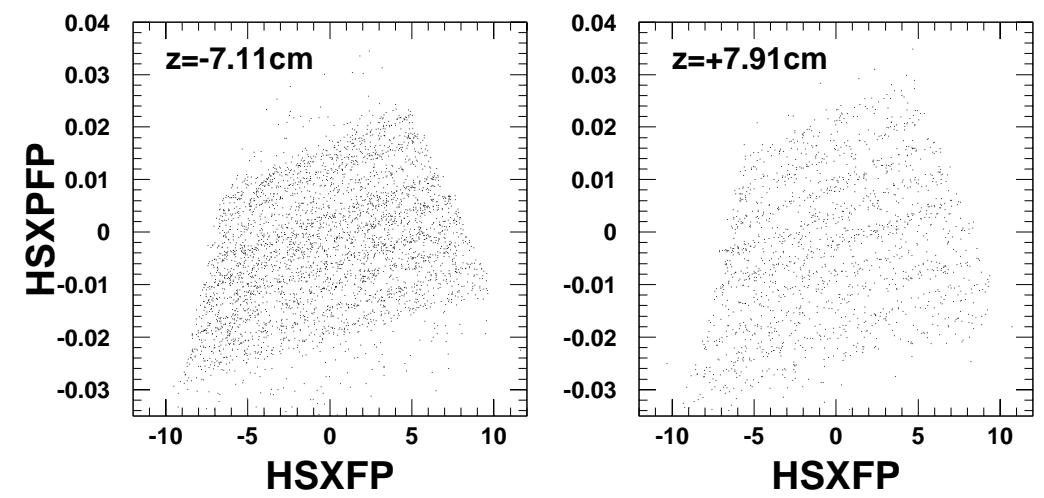

Figure 3.6: $H M S x_{f p}^{\prime}$ vs $x_{f p}$ for the two outermost quintar targets. The angle for the outermost sieve holes varies by $\approx 7$ mrad for foils at $z=+7.91$ (-7.11) cm relative to the one at $z=0$. The change in angle leads to a non-zero vertical beam position in the HMS optical plane $\left(x_{t a r} \neq 0\right)$, which in turn affects the reconstructed variables.

While taking the $z$ position of the quintar foils into account in the reconstruc- 
tion is relatively straightforward, the effect of a vertical beam offset due to extended targets is not easily eliminated. Taking into account the beam position offset is complicated, because it is impossible to know the origin of an event along the beam a priori. The approach taken in this analysis, is to calculate the position of the target in the lab frame, $z_{t a r}$, for a given set of $y_{t a r}, y_{t a r}^{\prime}$ and $x_{t a r}^{\prime}$ using equation 3.6. The vertical beam position, $x_{t a r}$, can be calculated from,

$$
x_{\text {tar }}=-z_{\text {tar }} \cos (\theta) \times x_{\text {tar }}^{\prime}
$$

where $\theta$ is the spectrometer central angle. The correction to the reconstructed out of plane angle, $x_{t a r}^{\prime}$, can then be determined to linear order from equation 3.5. The result of this correction was significant for reconstruction of data taken with the carbon quintar target, but had no observable effect on the analysis of $\mathrm{F}_{\pi}$ production data.

\subsubsection{Spectrometer Acceptance}

The spectrometer acceptance is a function of the reconstructed quantities $x_{t a r}^{\prime}$, $y_{t a r}, y_{t a r}^{\prime}$ and $\delta$. Comparing the reconstructed quantities such as $y_{\text {tar }}^{\prime}$ to the corresponding quantitity simulated with a Monte Carlo as described in Chapter 4 provides a way to validate the spectrometer matrix elements and description of phase space. Under the assumption that the Monte Carlo model is accurate the boundaries of these distributions should match within uncertainty of the measurement.

The HMS acceptance was studied using elastic electron scattering on hydrogen 


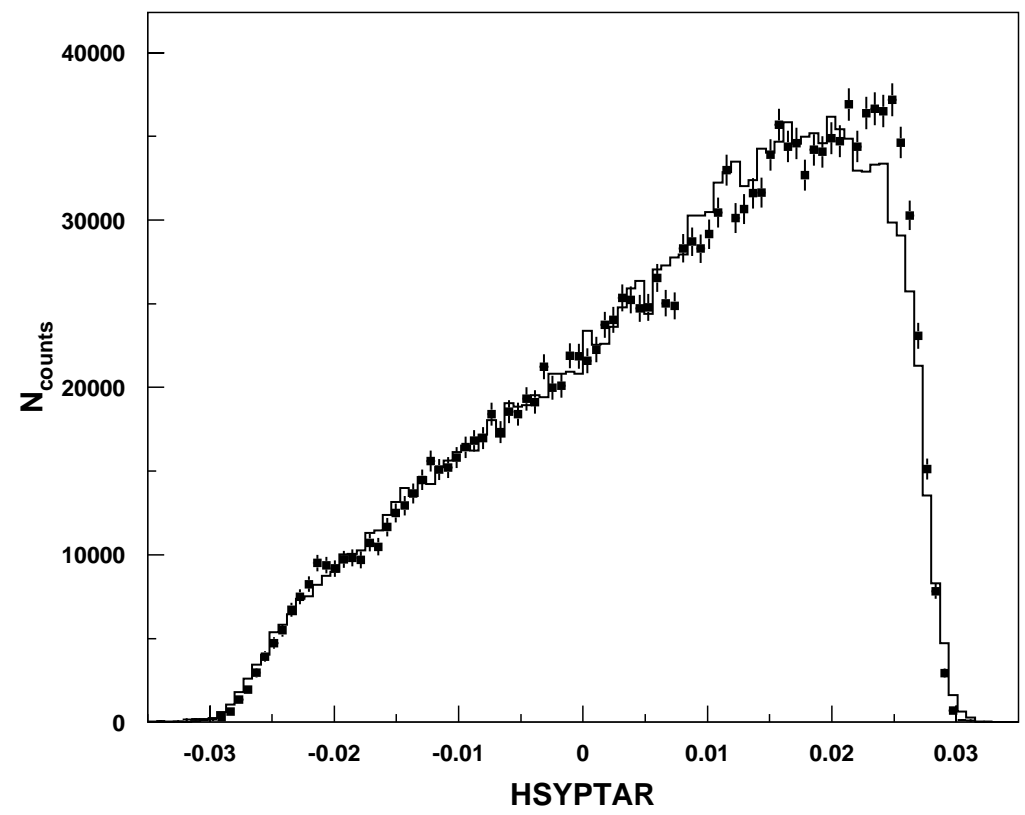

Figure 3.7: HMS $y_{\text {tar }}^{\prime}$ acceptance for elastic $e-p$ data at $P_{H M S}=3.83 \mathrm{GeV} / \mathrm{c}$ and $\theta_{H M S}=12^{\circ}$. The left panel shows the distribution of data (crosses) and SIMC (solid line). The acceptance is generally described well except for small regions at the edges. While this discrepancy is likely of no major concern if one integrates over this region, it is important in this analysis due to the direct correlation with $-t$.

comparing the limits of the acceptance for reconstructed $x_{t a r}^{\prime}, y_{t a r}, y_{t a r}^{\prime}$ and $\delta$ for experimental and simulated data. While the acceptance model performs generally quite well over the phase space covered there are small regions in reconstructed $y_{\text {tar }}^{\prime}$ with significant disagreement between experiment and simulation $(\approx \pm 25 \mathrm{mrad}$ as illustrated in Figure 3.7). This disagreement was also observed in previous experiments [81]. In this analysis the full acceptance was studied from deep inelastic electron scattering on deuterium. The disagreement was attributed to a pathological behaviour of the HMS matrix elements at extreme corners of phase space. The part of phase space for which the disagreement between boundaries was observed most clearly was analyzed in detail during $\mathrm{F}_{\pi}-2$ analysis. In particular, the study of selected regions in the focal plane revealed a dependence of reconstructed $y_{t a r}^{\prime}$ on 
horizontal position which is in turn related to the fractional momentum, $\delta$. The apparent momentum dependence of the reconstructed phase space may be interpreted in terms of the uncertainty in the beam position during the calibration of the initial HMS matrix elements in 1997. Since the spectrometer in-plane angle is directly related to the horizontal beam position from the center of the target, the resulting reconstruction matrix will be fitted with respect to this reference point. The horizontal beam position during calibration is assumed to be centered at zero and subsequent experiments generally use this guideline in determining the individual beam positions. An uncertainty of $0.2 \mathrm{~mm}$ in the beam position results in a global modification of the in-plane-angle of $\approx 0.23 \mathrm{mrad}$, which in turn can affect particular regions of the momentum reconstruction. To address the observed disagreement of the $y_{t a r}^{\prime}$ boundaries in parts of the phase space the reconstruction matrix elements were optimized including the measured horizontal beam position. The analysis of both elastic and $\mathrm{H}\left(e, e^{\prime} \pi^{+}\right) \mathrm{n}$ data showed an improved agreement between data and simulation using the optimized matrix elements. However, the data were still not described entirely by the simulation.

The coincidence dynamic phase space between HMS and SOS was analyzed using $\mathrm{H}\left(e, e^{\prime} \pi^{+}\right) \mathrm{n}$ data and comparing physics quantities such as invariant mass, momentum transfer, missing mass and momentum distributions. In addition, to study the physics dependence on particular regions of phase space the correlations between kinematic and reconstructed spectrometer spectrometer quantities were examined. Two effects were observed, which were corrected for in the reconstruction of the target variables. First, a dependence of reconstructed fractional momentum, 
$\delta$, in the SOS on $x_{f p}^{\prime}$ was found. This effect is attributable to saturation effects from the SOS resisitive magnets and was observed and corrected for in previous experiments [56]. However, residual correlations were observed at SOS momenta above $>1.7 \mathrm{GeV} / \mathrm{c}$. To eliminate these correlations a new correction was fit using elastic coincidence data.

A second correlation was found between missing mass and HMS $y_{f p}^{\prime}(\mathrm{a} \pm$ 1.5 MeV effect). A similar correlation was observed in elastic $\left(e, e^{\prime}\right)$ data. This effect is attributable to the presence of "forbidden" matrix elements in the HMS reconstruction matrix. The effect is corrected by fitting an ad-hoc correction of the form

$$
\delta_{\text {corrected }}=\delta+C_{\delta}\left(x_{f p}^{\prime}\right) \times y_{f p}^{\prime}
$$

The correction factor, $C_{\delta}\left(x_{f p}^{\prime}\right)$ is fit for a number of selected intervals in $x_{f p}^{\prime}$ for all $\mathrm{H}\left(e, e^{\prime} \pi^{+}\right)$kinematic settings and both $Q^{2}$ settings and the $x_{f p}^{\prime}$ dependence is parametrized by a continous function (see Figure 3.9). The parametrization in terms of $x_{f p}^{\prime}$ was chosen due to the angle dependence of phase space covered in the focal plane for pion production data. It should be noted that elastic data were not suitable for fitting a correction due to limited coverage in $y_{f p}^{\prime}$. The correction is applied to the reconstruction of all data and eliminates most of the observed correlation. The observed effect is likely the result of the known Q3 current offset and can be modeled in the Hall $\mathrm{C}$ Monte Carlo. Details of the modeling of the effect can be found in Chapter 4. It should be noted that the only strong correlation between the HMS and SOS angle coordinates is given in terms of the fractional momenta. This excludes an 


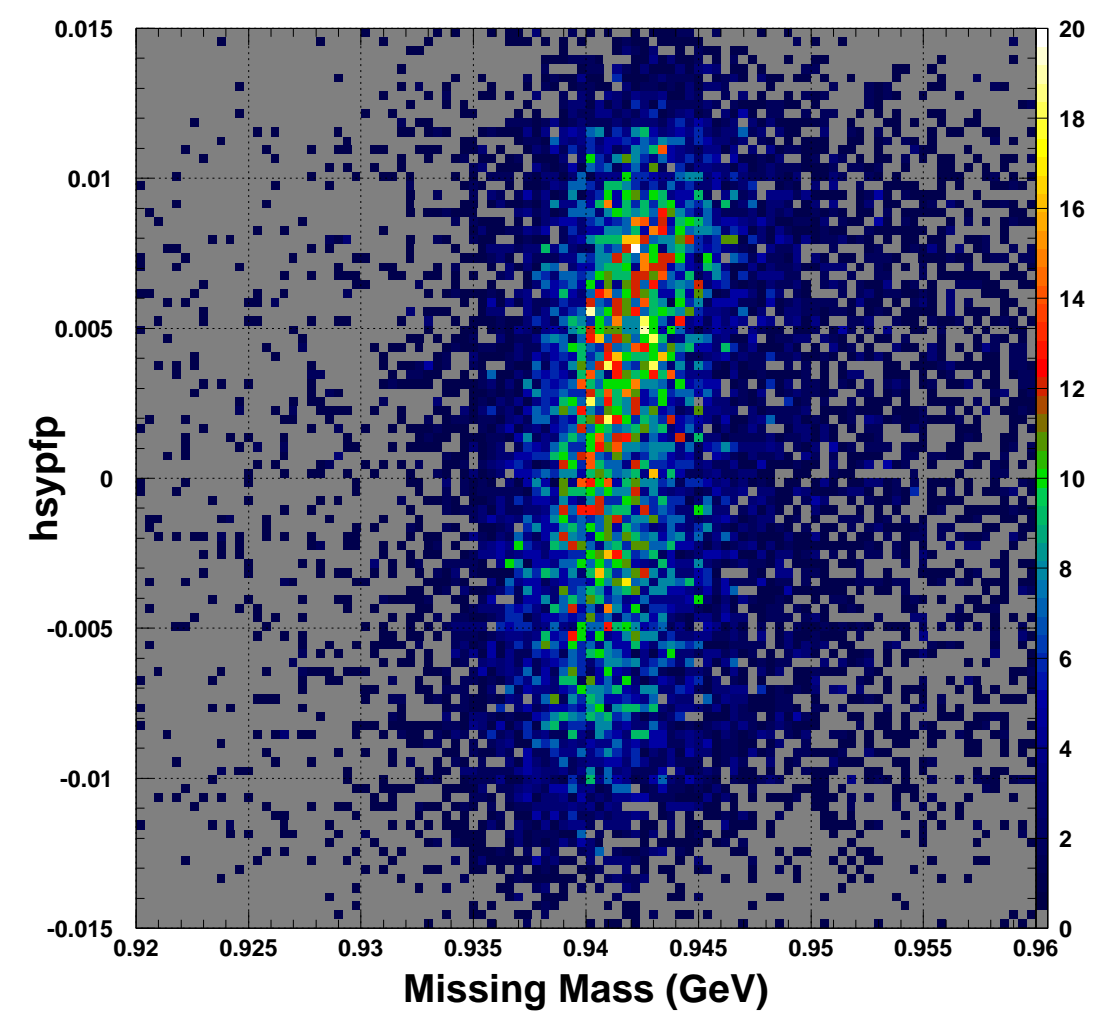

Figure 3.8: Reconstructed missing mass from $H\left(e, e^{\prime} \pi^{+}\right) n$ data plotted vs. HMS $y_{f p}^{\prime}$ (hsypfp) at $P_{H M S}=2.93 \mathrm{GeV} / \mathrm{c}$. The missing mass should reconstruct to the neutron mass independent of target and focal plane variables. However, the missing mass centroid varies by $1.5 \mathrm{MeV}$ across the hsypfp acceptance. A similar effect is observed in elastic data, where the invariant mass is studied as a function of hsypfp. To eliminate this correlation an ad-hoc correction is fit using $H\left(e, e^{\prime} \pi^{+}\right) n$ data (see text).

explanation for the observed missing mass- $y_{f p}^{\prime}$ correlation in terms of a SOS $\delta-x_{f p}^{\prime}$ dependence.

\subsection{Experimental Offsets}

Experimental offsets result from uncertainties in the reconstructed quantities that are not taken into account with the optimization of the spectrometer matrix 


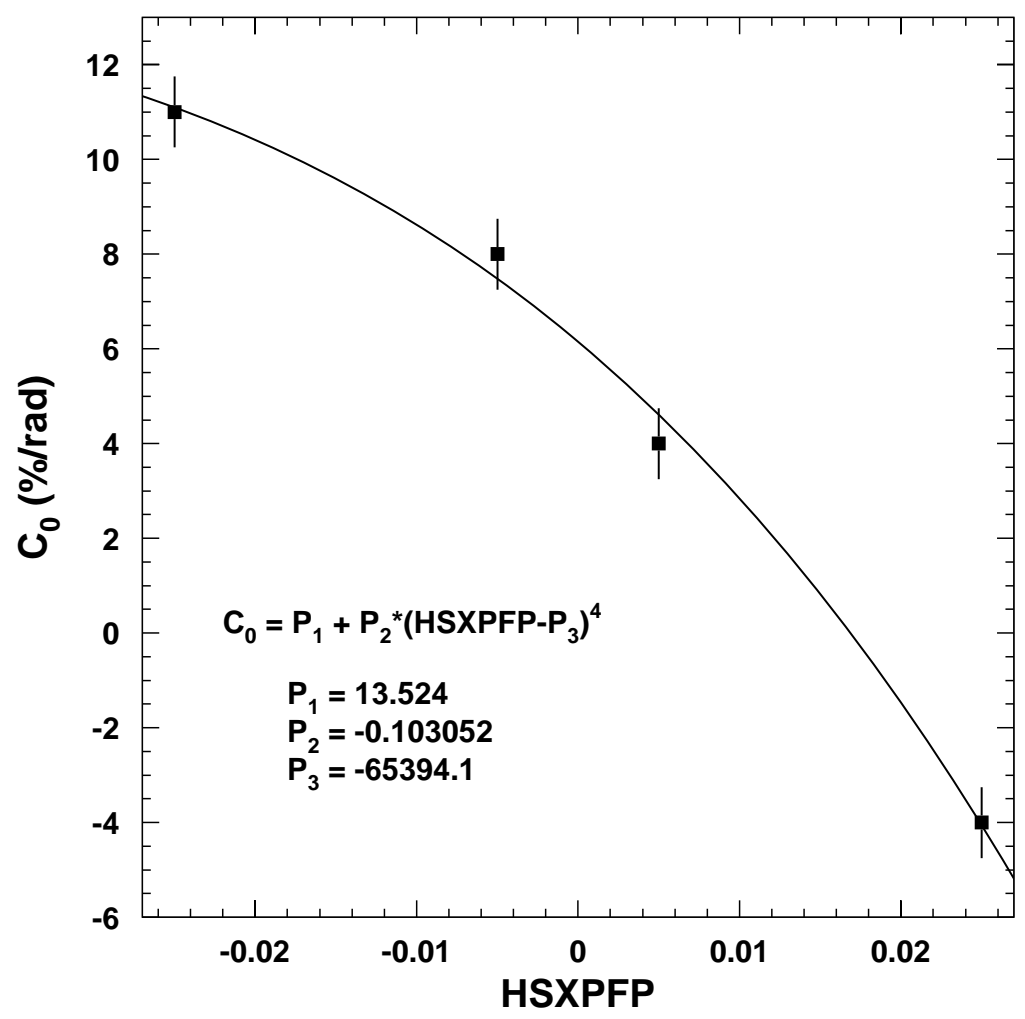

Figure 3.9: HMS ( $\delta$, hsypfp) correction $\left(C_{\delta}\right)$ as a function of HMS xpfp.

elements. Examples of such uncertainties are saturation and current offset effects in the spectrometer magnets and are typically small in size. The calculation of the experimental cross sections depends on physics quantities like $\mathrm{W}, \mathrm{Q}^{2}$ and $\mathrm{t}$ obtained from reconstructed spectrometer quantities (central angles and momenta) and the beam energy and therefore results in a direct sensitivity to uncertainties in the spectrometer quantities. The uncertainty in the resulting cross section can be expressed in terms of the "kinematic offsets", which take into account the deviation of a quantitiy such as the spectrometer central angle from its nominal value during the experiment. 


\subsubsection{Kinematic Offsets}

The preferred set of experimental offsets is defined as that which describes the kinematical quantities (e.g. elastic invariant mass, $W$ ) within their uncertainties. The kinematic offsets were fit in two steps in this analysis. First, elastic electron singles data were used to fit angle and momentum offsets for the HMS assuming that the beam energy is known to $1-3 \times 10 \mathrm{GeV}^{-41}$. In fitting the offsets, the reconstructed invariant mass $(W)$ is compared to the proton mass and the effect of radiation and energy loss is taken into account. The effect of the beam positions not being centered vertically must be included as well, because such an offset can mimic a momentum offset (see section 3.2.1). If the experimental offsets are taken into account properly or if there are no offsets, the invariant mass is equal to the proton mass independent of HMS angle and momentum. However, a significant dependence of $W$ on the HMS central angle, $\theta_{H M S}$, was found at small scattering angles. This dependence is illustrated in the upper panel of Figure 3.10. The difference between $W$ and proton mass is shown as a function of HMS scattering angle for elastic $e-p$ data for $\delta=0$. The observed correlation is similar for other values of $\delta$. No significant sensitivity to the out of plane angle was found. Eliminating the correlation in $\theta_{H M S}$ requires both fitting HMS angle and central momentum offsets and taking into account correlations in reconstructed variables on focal plane quantities as described in section 3.1. The optimal set of $\mathrm{F}_{\pi^{-}}-2$ HMS experimental offsets is shown in Table 3.2 and includes no offset in the HMS central angle (compared to

\footnotetext{
${ }^{1}$ Though it is possible to let the beam energy vary in the fitting procedure, it is not necessary given the level of accuracy in the beam energy measurement.
} 


\begin{tabular}{||l|c|c|c|c||}
\hline $\begin{array}{l}E_{e} \\
(\mathrm{GeV})\end{array}$ & $\begin{array}{c}p_{e} \\
(\mathrm{GeV} / \mathrm{c})\end{array}$ & $\begin{array}{c}\theta_{e} \\
(\mathrm{deg})\end{array}$ & $\begin{array}{c}p_{p} \\
(\mathrm{GeV} / \mathrm{c})\end{array}$ & $\begin{array}{c}\theta_{p} \\
(\mathrm{deg})\end{array}$ \\
\hline 4.210 & 1.581 & 51.00 & 3.442 & 20.90 \\
5.246 & 1.740 & 50.01 & 4.335 & 18.00 \\
3.779 & 1.420 & 54.00 & 3.154 & 21.40 \\
4.709 & 1.740 & 48.01 & 3.779 & 20.50 \\
\hline
\end{tabular}

Table 3.1: Experimental settings for the $F_{\pi}$-2 elastic coincidence runs. Electrons were detected in the SOS and protons were detected in the HMS.

the previously used angle offset of $1 \mathrm{mrad}$ ). The $\mathrm{F}_{\pi}$ HMS kinematic offsets are also in relatively good agreement with elastic electron singles data from 1999 [89] and from data taken in 2004 [71].
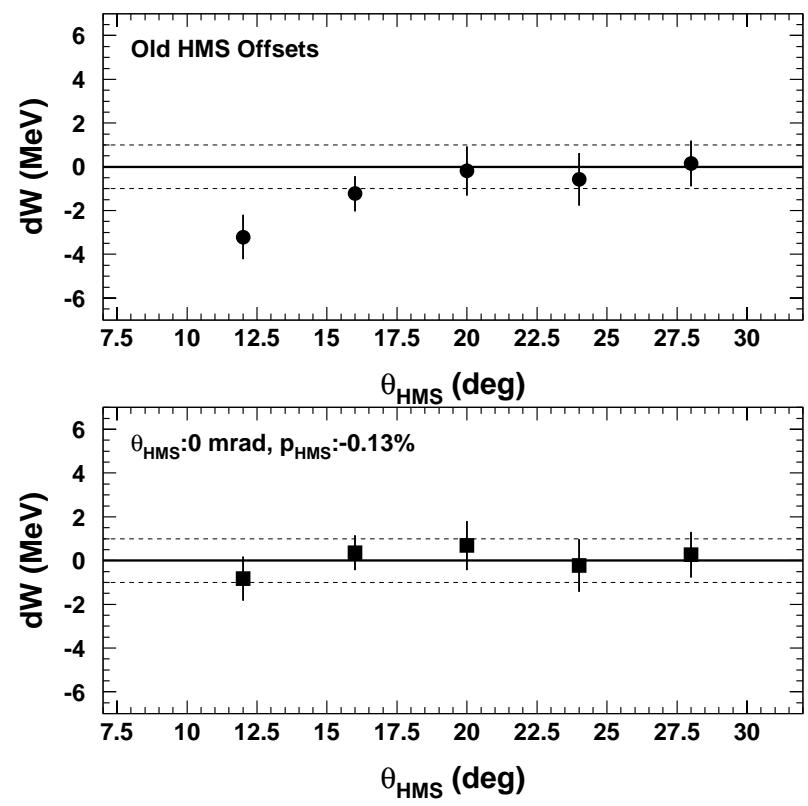

Figure 3.10: HMS central angle offset study using ep elastic data. The difference between invariant mass and proton mass is shown as a function of HMS angle for two sets of kinematic offsets. The linear correlation at forward angles in the upper panel was determined to be partially due to a 1 mrad offset in the HMS central angle. However, to completely eliminate the correlation, the $x_{f}^{\prime} p$ correction to HMS $\delta$ (see section 4.3 has to be taken into account as well.

The full set of experimental offsets including the SOS offsets makes use of the 
overconstrained $\mathrm{H}\left(e, e^{\prime} p\right)$ reaction in combination with the optimal set of offsets determined from the HMS. In this procedure the deviation of invariant reconstructed mass, missing energy, and missing momentum from their nominal values are examined. Analogous to the $\mathrm{H}\left(e, e^{\prime}\right)$ reaction, the invariant mass of the photon-target system should equal the proton mass. The missing energy in the $\mathrm{H}\left(e, e^{\prime} p\right)$ can be expressed as

$$
E_{m}=E_{e}-E_{e^{\prime}}-T_{p},
$$

where $E_{e}$ and $E_{e^{\prime}}$ are the energies of the incoming and scattered electron and $T_{p}$ is the kinetic energy of the recoiling proton. All particles are detected in this reaction, so the missing energy should be zero. The components of the missing momentum, $\mathbf{p}_{\mathbf{m}}=\mathbf{p}_{e}-\mathbf{p}_{e^{\prime}}$ are defined in terms of the components parallel and perpendicular to the three momentum transfer in the scattering plane as well as the out-of-plane component. In the $\mathrm{H}\left(e, e^{\prime} p\right)$ reaction all components of the missing momentum should be zero. The missing momentum is of particular interest in this experiment, because the momentum transfer to the nucleon, $t$, and $\phi_{p i}$ (see Figure 1.10) depend on its reconstruction. The entire set of experimental spectrometer quantities consists then of the beam energy, $E_{e}$, the spectrometer central momenta, $P_{e}$ and $P_{p}$, the spectrometer central angles, $\theta_{e}$ and $\theta_{p}$ and the out-of-plane spectrometer angles $\phi_{e}$ and $\phi_{p}$. The subscripts $e$ and $p$ refer to the scattered electron and recoiling proton respectively. The out of plane component of the missing momentum $\left(p_{m}^{o o p}\right.$ is sensitive to $\phi_{e}$ and $\phi_{p}$, while the other momentum components, $E_{m}$ and $W$ are sensitive to the remaining experimental quantities. For the optimization of the experimental offsets 
a standalone routine, called Heepcheck, was used. In this routine, the minimal deviation of the reconstructed quantities from their nominal values via the derivatives of each quantities with respect to all spectrometer quantities is determined. Further detail on the Heepcheck program can be found in reference [88]. During $\mathrm{F}_{\pi^{-}}-2$, $\mathrm{H}\left(e, e^{\prime} p\right)$ data were taken at each kinematic setting (Table 3.1) and the kinematic offsets were determined using the method outlined above. Assuming effectively no offset in beam energy and using the constraints from the kinematic offsets determined from the HMS, a momentum dependent correction due to saturation and no central angle offset were found for the SOS.

During the experiment the vertical nominal beam position was determined using the requirement that the reconstructed $x^{\prime}=0$ for the central hole of the HMS sieve slit. This requirement ensures that the beam position is consistent with the position at which the HMS reconstruction matrix elements were fit. Effectively one defines in this way the " $y=0 "$ point of the beam in the lab coordinate system. In the ideal case this point would coincide with the surveyed value " $y=0$ ", which is such that the HMS has no sagging and the SOS optical axis lies +2.6 mrad below the optical axis. However, experimental data suggest that this is not the case and $x^{\prime}$ does not reconstruct to $x^{\prime}=0$, thus the true vertical offsets are different from the ones above. This inconsistency may be attributed to a small beam position offset not taken into account when the HMS reconstruction matrix was fit in 1999 [82]. The true vertical offsets for HMS and SOS consistent with $\mathrm{y}=0$ can be determined using elastic coincidence data. Note that there is no rigid constraint on the choice of the vertical optical axis $\left(x^{\prime}=0\right)$, and for practical purposes it is sufficient to let 
the HMS define the appropriate vertical beam position.

In the $\mathrm{F}_{\pi}-2$ analysis HMS and SOS $x_{t a r}^{\prime}$ offsets were separated by looking at the out-of-plane momentum difference. Since the vertical beam position significantly influences the reconstruction of $x_{t a r}^{\prime}$ (see section 3.2.1), any offset in the beam position has to be taken into account in this method. The relation between HMS and SOS out-of-plane angles can then be written,

$$
x_{S O S}^{\prime}-\left(0.43-1.14 \frac{P_{p}}{P_{e}}\right) \cdot D_{b}+\frac{P_{p}}{P_{e}} x_{H M S}^{\prime}=0
$$

where $P_{e}$ and $P_{p}$ are the electron and proton momentum given by the spectrometer central momentum and $D_{b}$ is the vertical offset of the beam in mm. $x_{H M S}^{\prime}$ and $x_{S O S}^{\prime}$ are the reconstructed out-of-plane angles. The values $1.14 \mathrm{mrad} / \mathrm{mm}$ and 0.43 $\mathrm{mrad} / \mathrm{mm}$ are the first order HMS and SOS expansion coefficients denoting the effect of the vertical beam position on $x_{t a r}^{\prime}$. For each setting the vertical beam position was determined from optics sieve slit data. Using equation 3.10 one can separate HMS and SOS offsets,

$$
x_{S O S}^{\prime}+\frac{P_{p}}{P_{e}} x_{H M S}^{\prime}=\left(0.43 D_{b}-d \phi_{S O S}\right)-\left(1.14 D_{b}+d \phi_{H M S}\right)\left(\frac{P_{p}}{P_{e}}\right),
$$

where $d \phi_{H M S}$ and $d \phi_{S O S}$ denote the vertical offsets for HMS and SOS. The results of this analysis are shown in Figure 3.11. The entire set of kinematic offsets for $\mathrm{F}_{\pi}-2$ is listed in Table 3.2.

After the set of experimental offsets was determined it was found that the miss- 


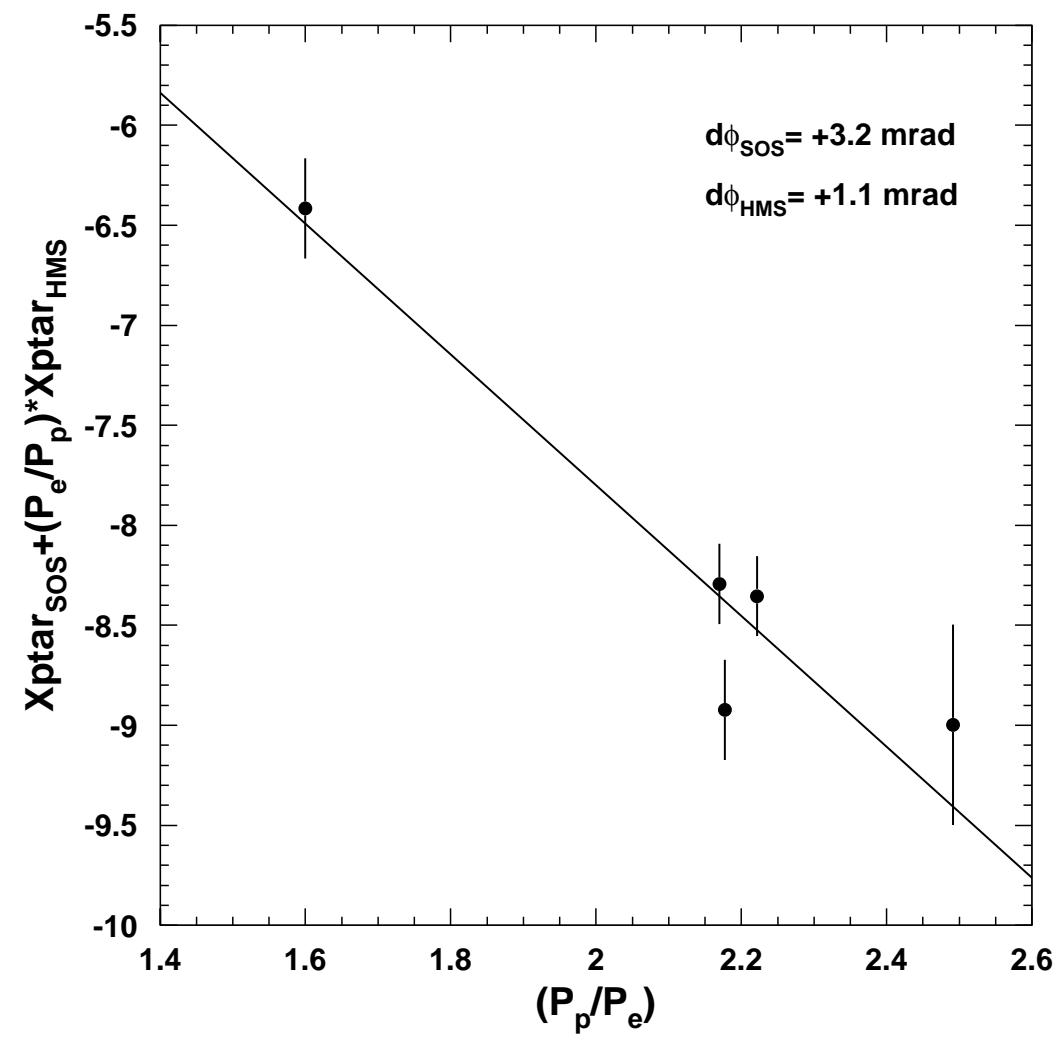

Figure 3.11: Out of plane Offsets determined from elastic coincidence data. The fraction of electron and proton momentum is calculated from the spectrometer central momenta. The values on the vertical axis include the offset of the vertical beam position at each kinematic setting. The vertical beam position is determined from sieve slit data and BPM information in this analysis.

ing mass in the $\mathrm{H}\left(e, e^{\prime} \pi^{+}\right)$n reaction did not reconstruct to the neutron mass for two settings ( $\mathrm{a} \approx \pm 2 \mathrm{MeV}$ effect). The missing mass depends predominantly on electron momentum and electron beam energy and the origin of the observed disagreement was explained in terms of these quantities. In the first case the SOS momentum was set to $1.65(\mathrm{GeV} / \mathrm{c})$, a setting far in the saturation region of the magnets. Due to the rapid increase of the saturation effect in this region the parametrization function includes relatively large uncertainties. To correct the observed shift in missing mass 


\begin{tabular}{||l|c|c|}
\hline Quantity & HMS & SOS \\
\hline$\theta$ & - & - \\
$\phi$ & $+1.1 \mathrm{mrad}$ & $+3.2 \mathrm{mrad}$ \\
$p_{0}$ & $-0.13 \%$ & $0.0-1.4 \%$ \\
\hline$E_{e}$ & - & - \\
\hline
\end{tabular}

Table 3.2: Kinematic offsets measured in $F_{\pi}$-2.

at this setting a correction of $+0.075 \%$ was applied to the SOS momentum offset. In the second case the shift in neutron mass was corrected in terms of a beam energy offset of $+0.05 \%$. The beam energy offset was determined from an analysis of the relative beam energy over the course of the run and was attributed to small drifts of the magnetic elements in the arc.

\subsubsection{Fast Raster Correction}

The vertical beam position at the target influences the reconstruction of the spectrometer fractional momentum, $\delta$, and the reconstructed out of plane angle, $x_{t a r}^{\prime}$. As indicated in section 3.2.1, the effect on $\delta$ can be susbtantial and must be taken into account. For example, a vertical beam position of $+1 \mathrm{~mm}$, would result in a momentum offset of $+0.077 \%$, a $0.1 \%$ effect.

In the $\mathrm{F}_{\pi}-2$ analysis, the vertical beam position is determined from the fast raster information. Corrections for both vertical motion of the raster and movement of the centroid of the beam are included. A good way to check for the effect of vertical beam offsets is to use elastic coincidence data and look for correlations between reconstructed missing energy and vertical fast raster position. Such a correlation was found in this analysis (see Figure 3.12 and was also observed in previous experiments 
[90]. While the size of the phase space decreases with decreasing beam energy the slope of the correlation remains. There could be at least two reasons for not correcting properly for vertical fast raster position. First, there could be a problem directly associated with the fast raster ADC signal used in the fast raster correction in the analyzer, secondly the fast raster calibration may not be quite accurate.

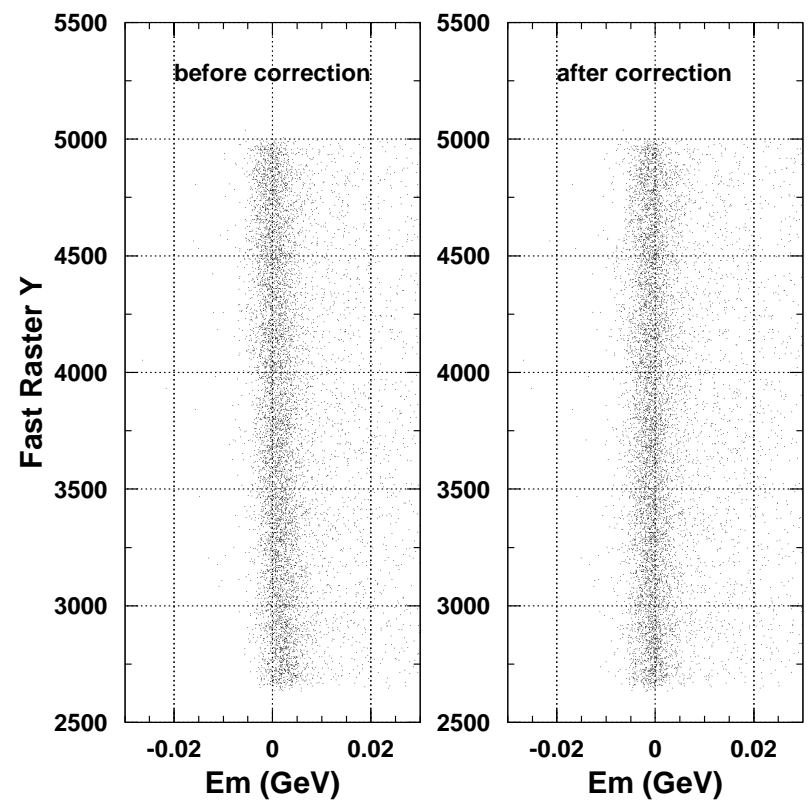

Figure 3.12: Fast raster $Y$ position (ie. vertical beam position) as a function of missing energy for elastic coincidence data before and after correcting the amplitude.

In the Hall $\mathrm{C}$ ENGINE the fast raster correction is applied to the vertical/horizontal beam positions. The vertical beam (and also raster) position used in the reconstruction is then determined from the FR corrected value. The fast raster calibration used for $\mathrm{F}_{\pi^{-}}$is from Aug, 1996. A significant deviation from the default calibration was found in subsequent experiments in 2004 [71]. In this case the FR calibration was updated using harp scan data taken at $\mathrm{P}_{H M S}=2.0 \mathrm{GeV}$. In 
order to correct the observed kinematics correlations with $\mathrm{FR} \mathrm{Y}$ in $\mathrm{F}_{\pi^{-}}-2 \mathrm{H}\left(e, e^{\prime} p\right)$ data the beam/raster position was multiplied by a constant factor of 1.5, implying a correction to the amplitude of $\approx 50 \%$.

\subsection{Particle Identification and Event Selection}

\subsubsection{Time of Flight}

The particle velocity determined from the time of flight information is generally an important element in the selection of events from the reaction of interest. The particle velocity is calculated from the timing information from the four scintillator hodoscope arrays in each spectrometer. The raw time that a particular scintillator element was hit is registered relative to a common start. The raw time is then corrected for pulse height and shape variation, propagation time of the scintillator light through the paddle to reach the PMT and timing offsets due to differences in cable lengths and electronic delays. The pulse height correction ensures consistent timing independent of pulse height by setting a lower bound on the timing ADC signal.

The pulse height correction and propagation time are taken into account as follows. A pure sample of events of the particle type of interest is analyzed. In this analysis the corrections were fit using both a sample of pions at negative polarity and a sample of electrons. If the particle type is unambigously specified the particle velocity can be precisely calculated from the reconstructed particle momentum 
using,

$$
\beta_{p}=\frac{p}{E}=\frac{|p|}{\sqrt{|p|^{2}+m_{\pi}^{2}}} .
$$

Given the distance between the hit scintillator paddles the parameters for timing coefficients and offsets can be optimized so that the particle velocity obtained from the measured time of flight $\left(\beta_{T O F}\right)$ is consistent with the particle velocity calculated from the particle momentum. The initial time of flight calibration for $\mathrm{F}_{\pi^{-}}-2$ resulted

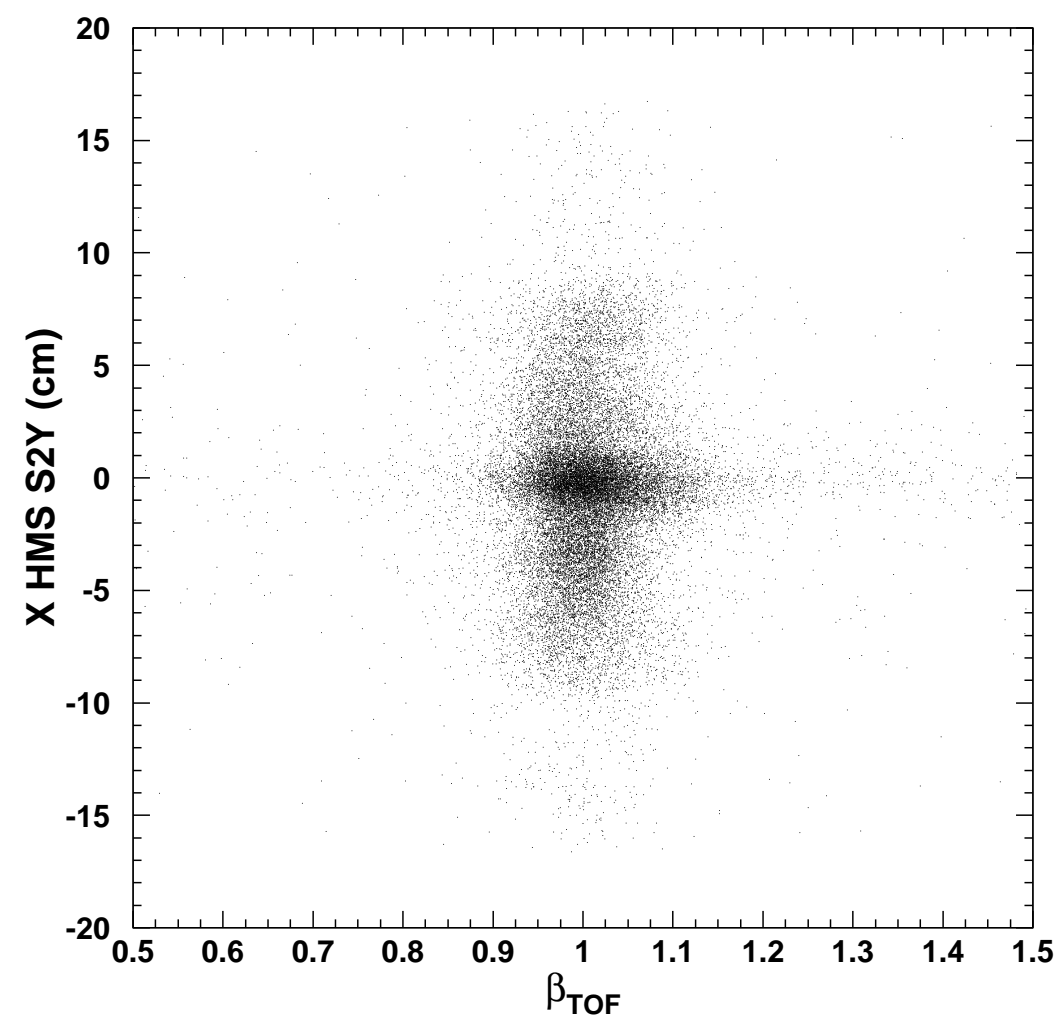

Figure 3.13: Position at the HMS S2Y hodoscope plane as a function of $\beta_{T O F}$. The hodoscope resolution is affected by erratic behavior of at least three paddles in the center of the plane. The resolution could be improved by eliminating a large fraction of the PMTs on one side of the plane from the time of flight calculation. The PMT signals were included on the trigger level, so that no good events were lost.

in poor timing resolution problems in the HMS hodoscopes after calibration. In 
addition, the projection of tracks to the location of the fourth scintillator layer revealed an offset of events near the hodoscope center. This was attributed to erratic behaviour of four PMTs in the fourth scintillator. Removing the erratic PMTs from the time of flight calculation in software resulted in a significant resolution improvement. The time of flight calculation uses signals from PMTs from both ends of the paddle so that the time of flight information can be retained even if PMTs are removed from only one side, as was done in this case.

\subsubsection{Electron Identification}

Electrons were identified in the SOS using a combination of the SOS gas Čerenkov and calorimeter. The gas Čerenkov was used as a threshold detector with a mean SOS signal of seven photoelectrons for one electron. Good electron events were selected for a photoelectron cut of $\mathrm{N}_{\text {photoelectrons }}>0.5$. This cut was chosen based on the position dependence of the mean photoelectron yield and to ensure good efficiency across the acceptance..

To determine the efficiency of the Čerenkov detector a pure electron sample was selected from elastic $\mathrm{H}\left(e, e^{\prime}\right)$ data using two SOS delta scans with SOS central momenta between $0.9-1.74(\mathrm{GeV} / \mathrm{c})^{2}$ and with scattering angles varying between 18 and 60 degrees. The corresponding delta range covers a region between $\pm 20 \%$. A tight cut on the invariant mass $(W)$ with a conservative cut on the calorimeter $(>0.7)$ removes all residual pion background. To obtain an unbiased set of events using only scintillator and calorimeter information, cuts were placed on the sELHI TDC $($ ELHI > 1000) so that the Čerenkov was not required at the trigger level. An 
additional restriction was placed on SOS $\delta$. The Čerenkov efficiency is then given by the ratio of events with and without the Cerenkov cut.

The efficiency for the accepted delta range was found to be at least $99.78 \%$ \pm (at central $\operatorname{SOS} \delta=+9 \%$ ) for a photoelectron cut of 0.5 (see Table 3.3) with a slight dependence on central momentum. However, no significant dependence of the efficiency across the acceptance was found. A typical spectrum of the efficiency accross the delta acceptance is shown in Figure 3.14. In the data analysis a cut efficiency of $99.92 \pm 0.02 \%$ was used. The pion rejection of the SOS Čerenkov on
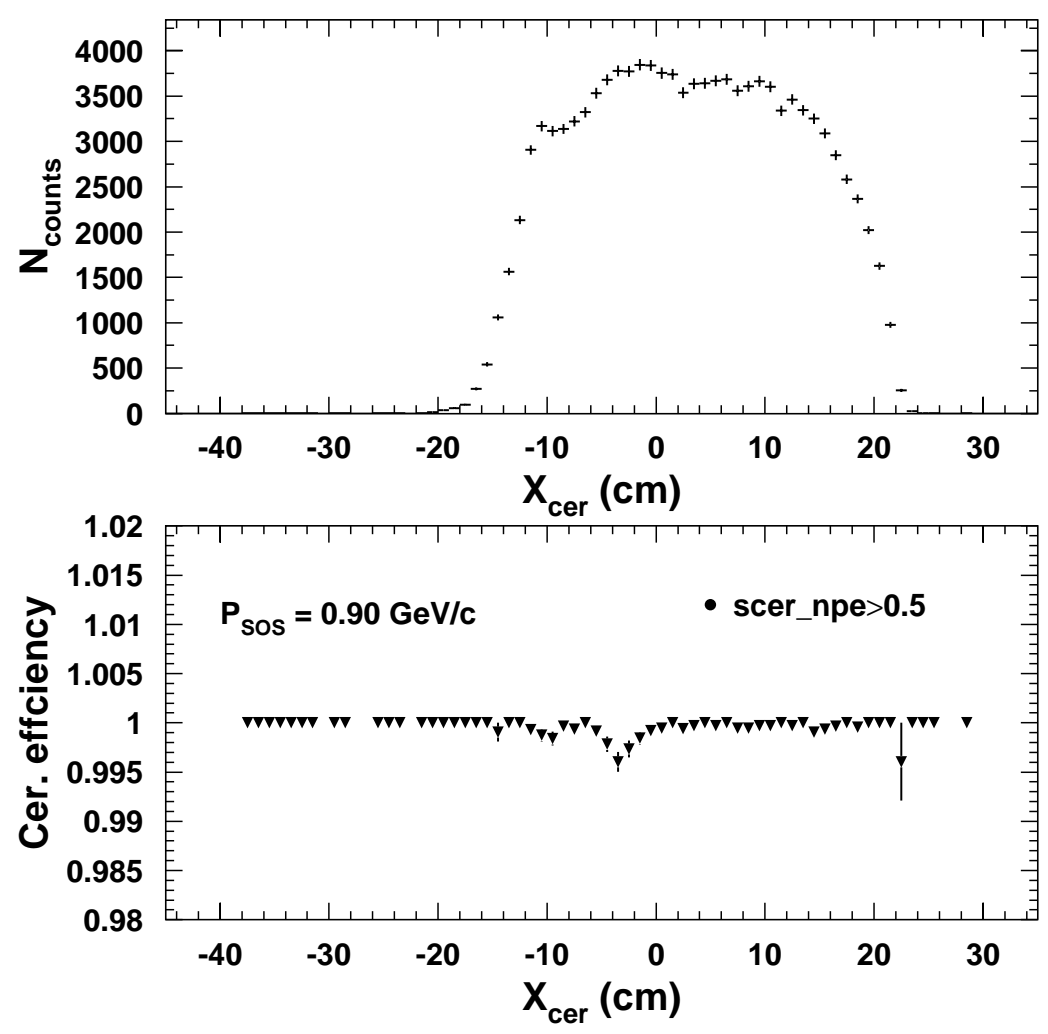

Figure 3.14: SOS Čerenkov efficiency.

the analysis level is influenced by the strength of photoelectron cut. In particular, 


\begin{tabular}{|l|c|c|}
\hline pSOS & SOS C electron efficiency & SOS calorimeter efficiency \\
\hline 0.90 & $99.93 \pm 0.01$ & $99.46 \pm 0.01$ \\
1.74 & $99.92 \pm 0.02$ & $99.47 \pm 0.03$ \\
\hline
\end{tabular}

Table 3.3: SOS Čerenkov and SOS calorimeter cut efficiencies. The electron cuts were $N_{\text {photoelectrons }}>0.5$ and $E_{\text {calorimeter }} / E^{\prime}>0.7$ for $F_{\pi}$-2.

the relatively low photoelectron cut used may lead to introducing significant pion contamination. As long as the resulting $\pi^{-} / \pi^{+}$coincidences are random and are removed by the subtraction of random coincidences this effect does not pose a problem. Unfortunately, $\mathrm{F}_{\pi}-2$ kinematics allow for a substantial fraction of real pion coincidences passing particle identification. However, the remaining pions can be effectively removed by requiring a cut on the SOS calorimeter in addition to the Čerenkov detector. The pion rejection for this cut was estimated using data taken with "open" (SCIN) trigger. Cuts were placed on the SOS Čerenkov and SOS time of flight and standard acceptance cuts were applied. The pion rejection is then the number of events passing the calorimeter cut over all events.

The efficiency of the calorimeter was determined in a similar fashion as the Čerenkov efficiency. A localized rate dependent inefficiency was initially found for the normalized and tracking based SOS calorimeter efficiency. This rate dependence could be attributed to a flaw in the analysis code. For the efficiency analysis SOS elastic $\mathrm{H}\left(e, e^{\prime}\right)$ data were used. Particle identification cuts were placed on the SOS Čerenkov and invariant mass in addition to loose cuts on SOS delta. In addition signals from trigger TDCs sELLO, sTOF and sSCIN were required. The last constraint is applied to eliminate the calorimeter in the trigger, which could result in 
biasing the electron sample studied. The efficiency is simply the ratio of events passing the calorimeter cut and the total number of events. The calorimeter efficiency is relatively constant for all SOS momenta and is independent of the acceptance. In the data analysis a lower cut of $E_{c a l} / E^{\prime}>0.7$ was used with a corresponding efficiency of $99.5 \pm 0.1 \%$ for all runs. The pion rejection factor for this cut is 1:20.

\subsubsection{Pion Identification}

Pions in the HMS are selected with the HMS aerogel Cerenkov at positive polarity and the HMS gas Čerenkov at negative polarity.

At negative polarity the gas Čerenkov detector is very efficient. Pions do not in principle produce a signal in the Čerenkov detector. However, there is a nonzero probability that pions will produce knock-on electrons while traversing the detector, which will result in a photoelectron number greater than zero. Applying a cut to reject electrons may then reject pion events as well. To determine the pion efficiency of the Čerenkov cut positive polarity $\pi^{+}$data was used. Cuts were placed on the reconstructed missing mass and on the calorimeter to eliminate positron background. An additional cut on the aerogel Cerenkov rejected protons. The ratio of events passing the cut to all events is then the Čerenkov pion efficiency.

The separation of pions from protons relies on the HMS aerogel Cerenkov. Whether or not a particle traversing the aerogel Čerenkov detector produces a signal depends on the index of refraction of the material and the particle velocity. The mean number of photoelectrons was 12 and stable to within $2 \%$ up to rates of 600

kHz. The average signal in the gas Cerenkov is 9-10 photoelectrons, but there are 
mirror regions at at the top of the detector where the average is only 4-6 photoelectrons. The local inefficiencies may be attributed to misalignment of the top mirror in the detector tank. Despite the misalignment the mean number of photoelectrons at the center of the acceptance was determind to be at least 8 photoelectrons. The pion Čerenkov efficiency is $99.6 \pm 0.05 \%$ for a cut of $\mathrm{N}_{\text {photoelectrons }}<2$.

The aerogel Čerenkov efficiency was determined from $\pi^{-}$production data with tight cuts on missing mass and calorimeter to eliminate electrons and on the aerogel Čerenkov to reject protons. For both HMS momentum settings the aerogel Čerenkov efficiency is $99.5 \pm 0.02 \%$ for a threshold cut of $\mathrm{N}_{\text {photoelectrons }}>3$.

To estimate the aerogel proton rejection to the efficiency elastic coincidence $\mathrm{H}\left(e, e^{\prime} \mathrm{p}\right)$ data were used. Cuts were placed on missing mass to eliminate positrons. The fraction of protons decreases with increasing threshold. However, since the fraction of protons that pass particle identification cuts is removed by the subtraction of random coincidences no further studies on proton rejection were done. Real proton coincidences are avoided via coincidence time cuts in this analysis.

\subsubsection{Subtraction of Backgrounds}

Random Coincidence

Random e- $\pi$ coincidence events constitute a background and have to be eliminated from the data sample. For this purpose the relative timing between HMS and SOS is used. In particular, the HMS coincidence trigger is timed by an HMS pretrigger starting the TDC and stopped by a delayed SOS coincidence trigger timed 

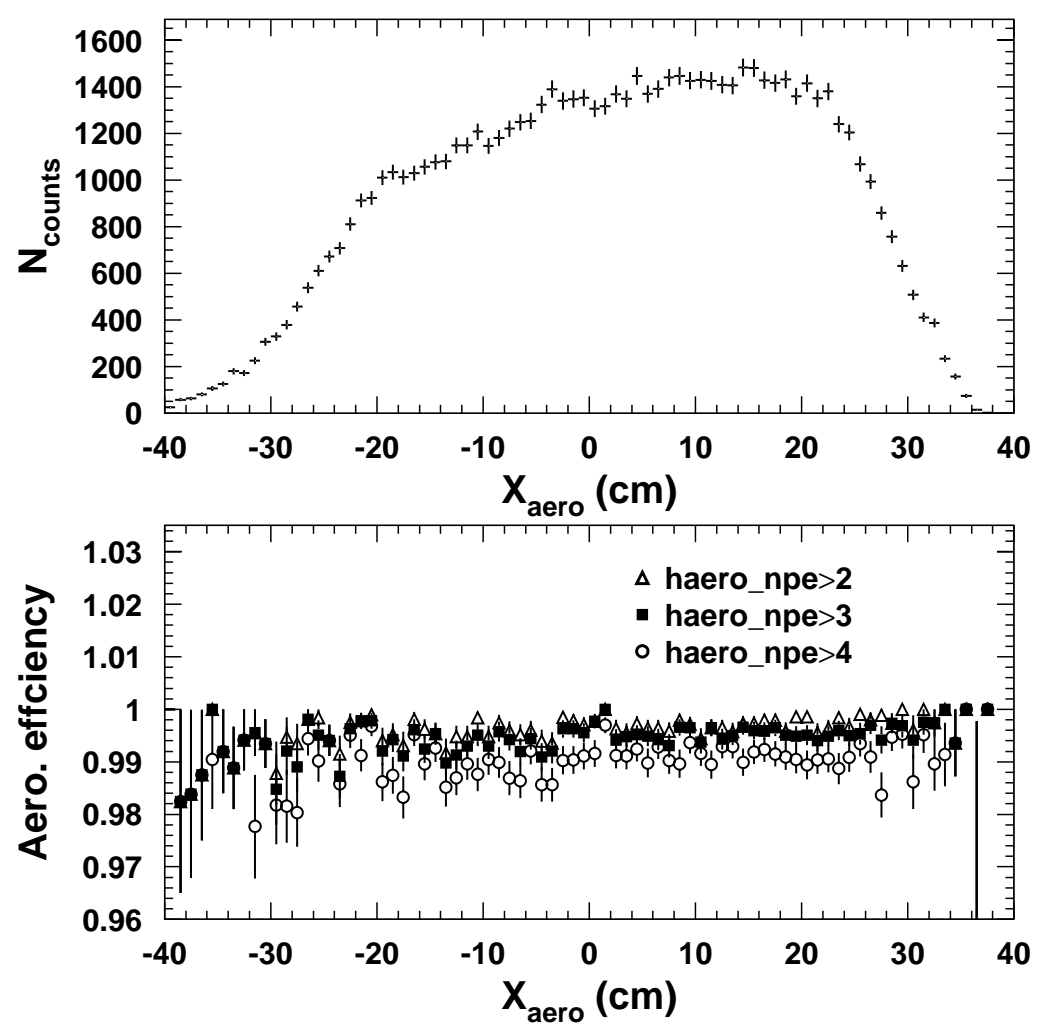

Figure 3.15: HMS aerogel Čerenkov detector horizontal acceptance and detector cut efficiency as a function of horizontal acceptance for three different photoelectron cuts. The cut efficiency for the cut used in this analysis is $99.5 \pm 0.02 \%$.

by the SOS. The time difference is the raw coincidence time. The raw coincidence time is corrected for time differences resulting from variations in particle velocity and pathlengths traveled through the spectrometer. Differences in pathlength arise from deviations of the particle trajectory from the central trajectory. Given the corrected coincidence time allows for a resolution of 200 ps, which allows for resolving the beam structure of the accelerator. A more detailed discussion of pathlength corrections is given in [62].

The implementation of the coincidence time cut is illustrated in Figure 3.16. 


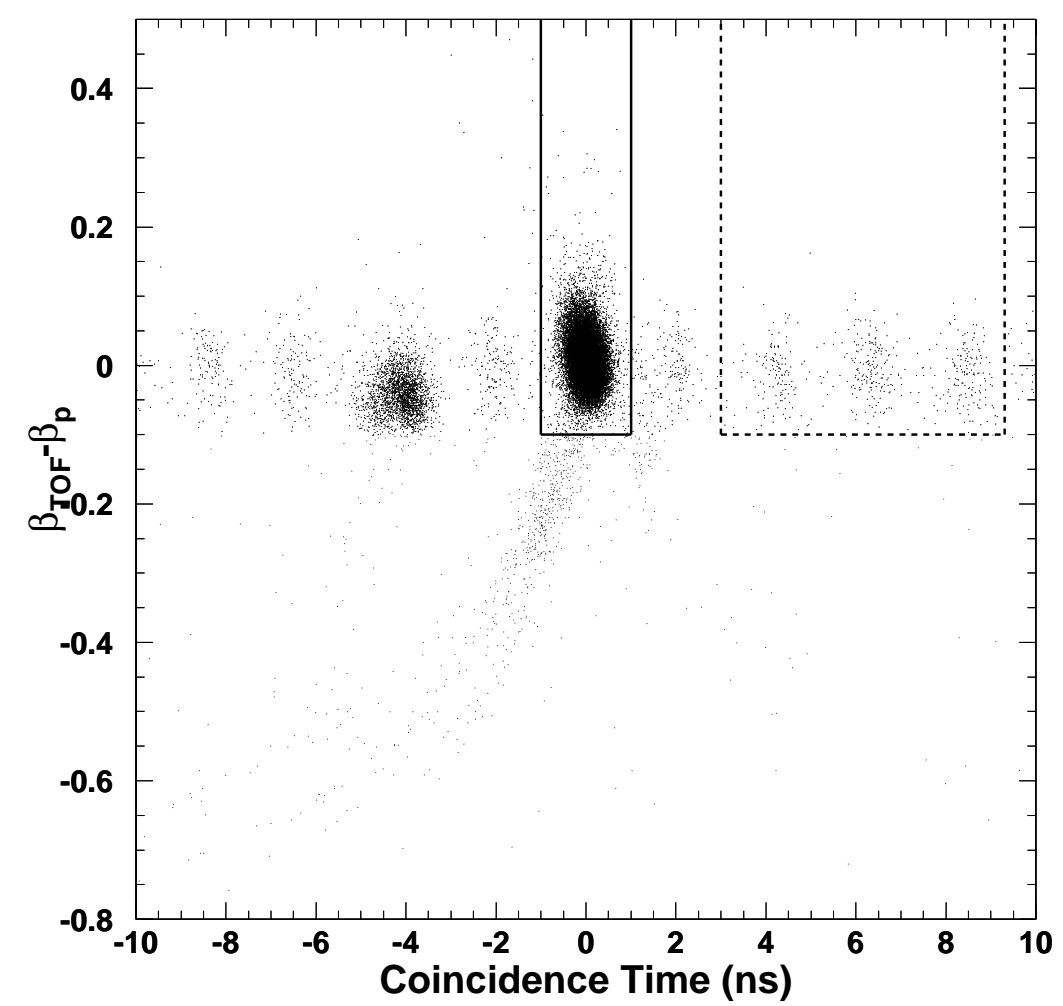

Figure 3.16: $F_{\pi}$-2 coincidence time spectrum as a function of $\beta_{T O F}-\beta_{p}$ including the real (solid) and random (dashed) coincidence time cuts. Real $e-\pi$ coincidences can be clearly identified and real proton coincidences are rejected by the coincidence time cut. The random coincidence background is $<1 \%$ for all kinematic settings. The "tail" in the time of flight spectrum at low $\beta-\beta_{p}$ results from pions interacting hadronically in the scintillator paddles. These events were eliminated to simplify the coincidence time cut. These lost "good" events must be accounted for in the efficiency calculation.

Real e- $\pi$ coincidences are selected with a cut at \pm 1 ns. To estimate the random background included in the "real" coincidence time peak, a sample of 3 "random" coincidence peaks was chosen. Real $e-p$ coincidences were avoided with these coincidence time cuts. The number of random events is then calculated as the average over the number of random peaks selected and subtracted from the number of events in the real coincidence peak. Note that there is a "tail" in the coincidence 
time spectrum at low $\beta-\beta_{p}$. These events result from pions undergoing nuclear interactions in the scintillators, Čerenkov or other material encountered in their path. The produced slower hadrons are identified as pions, but generally have a larger time of flight. Since pions traverse the drift chambers before interacting in subsequent materials, the resulting hadrons are valid tracks and reconstruct to good values of missing mass. To simplify the coincidence time cut, the low velocity hadron background was eliminated with a cut on the difference between the particle velocity determined from the time of flight and the velocity calculated based on the particle momentum. Since these events are associated to good pion tracks, events lost in this cut have to be taken into account by an additional global efficiency.

Besides misidentified pions, the time of flight spectrum identifies events for which no time of flight information can be found. These events appear at $\beta_{T O F}-$ $\beta_{P} \approx-1$, since missing time of flight information is denoted by $\beta_{T O F}=0$ in the Hall C ENGINE. There are at least two explanations for events with $\beta_{T O F}=0$. In the first case the analyzer fails to calculate the particle velocity because the projected location of the track at the relevant scintillator plane is outside the allowed area from the scintillator paddle. A second possibility is that events with no time of flight information are associated with pions interacting hadronically in the scintillators. The $\beta_{\text {TOF }}=0$ are eliminated in this analysis by the time of flight cut discussed above. The total fraction of "good" events lost are accounted for in the $\beta$ cut efficiency.

The $\beta_{\text {TOF }}-\beta_{P}$ cut efficiency was determined using $\pi^{-}$data. Cuts were placed on the gas Čerenkov to eliminate electrons and loose acceptance cuts were applied. The efficiency is then the fraction of events that pass the $\beta_{T O F}-\beta_{P}$ cut and the 
total number of events. The resulting cut efficiency for a cut $\beta_{T O F}-\beta_{P}>-0.1$ was determined to be $96.5 \pm 0.5 \%$ for all kinematic settings.

\section{Cell Wall Contribution}

Another type of background that has to be removed in the construction of a sample of good events is the background due to scattering from the aluminum target cell walls enclosing the cryogen. To estimate the contribution of these events the so-called dummy target is used. This target consists of two Aluminum foils placed at $\mathrm{z}= \pm 2 \mathrm{~cm}$. It should be noted that the dummy target foils are thicker by a factor of 7.773 than the nominal target cell walls resulting in higher luminosity and rapid measurement of the background.

The dummy target data are analyzed in the same way as the regular data including the same method of random coincidence subtraction and applying the same analysis cuts. The effective charge normalized yields are then subtracted from the real data yields taking into account the additional weight of 7.773 to account for the difference in wall thickness between target cell and dummy target.

The target cell wall contribution to the total measured yield $(2-4.5 \%)$ is relatively small, and taking into account the uncertainty in the ratio of thickness of target cell and dummy target, the contribution to the total uncertainty is negligible. In addition, the target cell wall contributions between $\epsilon$ settings is relatively stable, resulting in a negligible uncertainty in the separated cross sections. 


\subsection{Efficiency Corrections}

In forming the normalized yield, one must apply corrections for inefficiencies like track reconstruction and data acquisition deadtime. The total efficiency, $\epsilon_{\text {tot }}$, is applied to the total experimental yield as follows:

$$
Y_{\text {exp }}=\frac{N}{\epsilon_{t o t} Q_{t o t}}
$$

where $\mathrm{N}$ is the total number of events, $\epsilon_{\text {tot }}$ is the efficiency correction factor (all efficiencies combined) and $Q_{\text {tot }}$ is the total accumulated charge in $\mathrm{mC}$. The individual contributions to the efficiency factor are discussed in detail in the sections below.

\subsubsection{Trigger Efficiency}

The trigger in the HMS is largely determined by the scintillators, so that the associated efficiency of the scintillator signals is a direct measure of the overall trigger efficiency. Additional particle identification used during $\mathrm{F}_{\pi}-2$ includes the required absence of the Cerenkov signal in the HMS (pion trigger) and the presence of signals from calorimeter and Cerenkov in the SOS. The total trigger efficiency for the SOS is then given by the product of the efficiencies of the individual trigger components.

The 3 out of 4 efficiency is defined as the probability for three out of four signals from the four scintillator planes for a given event and can be expressed in 
terms of the individual plane efficiencies:

$$
\begin{aligned}
P_{3 / 4} & =P_{1} P_{2} P_{3} P_{4}+P_{1} P_{2} P_{3}\left(1-P_{4}\right)+P_{1} P_{2}\left(1-P_{3}\right) P_{4} \\
& +P_{1}\left(1-P_{2}\right) P_{3} P_{4}+\left(1-P_{1}\right) P_{2} P_{3} P_{4}
\end{aligned}
$$

where $P_{i}$ denotes the single plane efficiency for each scintillator plane. In principal, the individual plane efficiencies can be calculated from the number of times a valid track projected to the scintillator planes produces a signal in the paddle it intersects. However, as a result of multiple scattering the true particle trajectory may not always be identical with the reconstructed track and hence will result in an inaccurate calculation of the $3 / 4$ efficiency. The absolute measurement of the $3 / 4$ efficiency can be tested using the scintillator plane signals. To test the efficiency for a given plane for a given event we require that the other three planes must have registered a valid hit. Note that $3 / 4$ planes essentially means that a valid trigger is formed regardless of the response from the fourth plane. To minimize the track dependence of the efficiency adjacent paddles to the one that should have fired are included in the calculation as well. The $3 / 4$ efficiency calculation was performed for a subset of runs from each kinematic setting, target and polarity.

The $3 / 4$ efficiency across the spectrometer acceptance in a precision cross section measurement is of even greater interest than the absolute efficiencies. The acceptance dependence of the $3 / 4$ efficiency was studied using a parameterization of the individual scintillator paddle efficiencies. Using the calculated scintillator paddle efficiencies, the probability for a hit in a given plane can be determined. 
The $3 / 4$ efficiency is then calculated using equation 3.14 on an event by event basis $^{2}$. The HMS trigger efficiency was determined for both HMS ep elastic and pion electroproduction data at both polarities. In all cases a significant inefficiency was found at negative fractional momentum, $\delta$. A representative example is shown in Figure 3.17. For the $\mathrm{F}_{\pi}-2$ data analysis the rather large inefficiency does not pose a significant problem, because the experimental acceptance is limited to a region outside the affected acceptance region. In the analysis the inefficient region is eliminated by a cut on HMS $\delta$. The efficiency in the across the remaining part of the acceptance is $99.85 \pm 0.05 \%$.

\subsubsection{Tracking Efficiency and Multiple Tracks}

The tracking efficiency is defined as the ratio of events that should have passed through the drift chambers and the number of events for which a track was found. The fraction of events that should have passed through the drift chambers is defined by a requirement on hits in a fiducial area composed of a particular set of scintillator paddles. The efficiency depends on both the drift chamber hit efficiency and the tracking algorithm finding a track. In particular, it has been verified by previous experiments that the efficiency for tracking electrons in the HMS drift chambers falls off linearly with rate, which will be explained in terms of multiple track contribution.

To define a pure sample of the particle of interest for the calculation of the tracking efficiency relatively tight particle identification requirements are used. These requirements are stricter than the ones used in the regular analysis. The

\footnotetext{
${ }^{2}$ This method will yield a slightly lower (and) more accurate efficiency than the method described earlier since it accounts for correlations between planes for a given trajectory.
} 

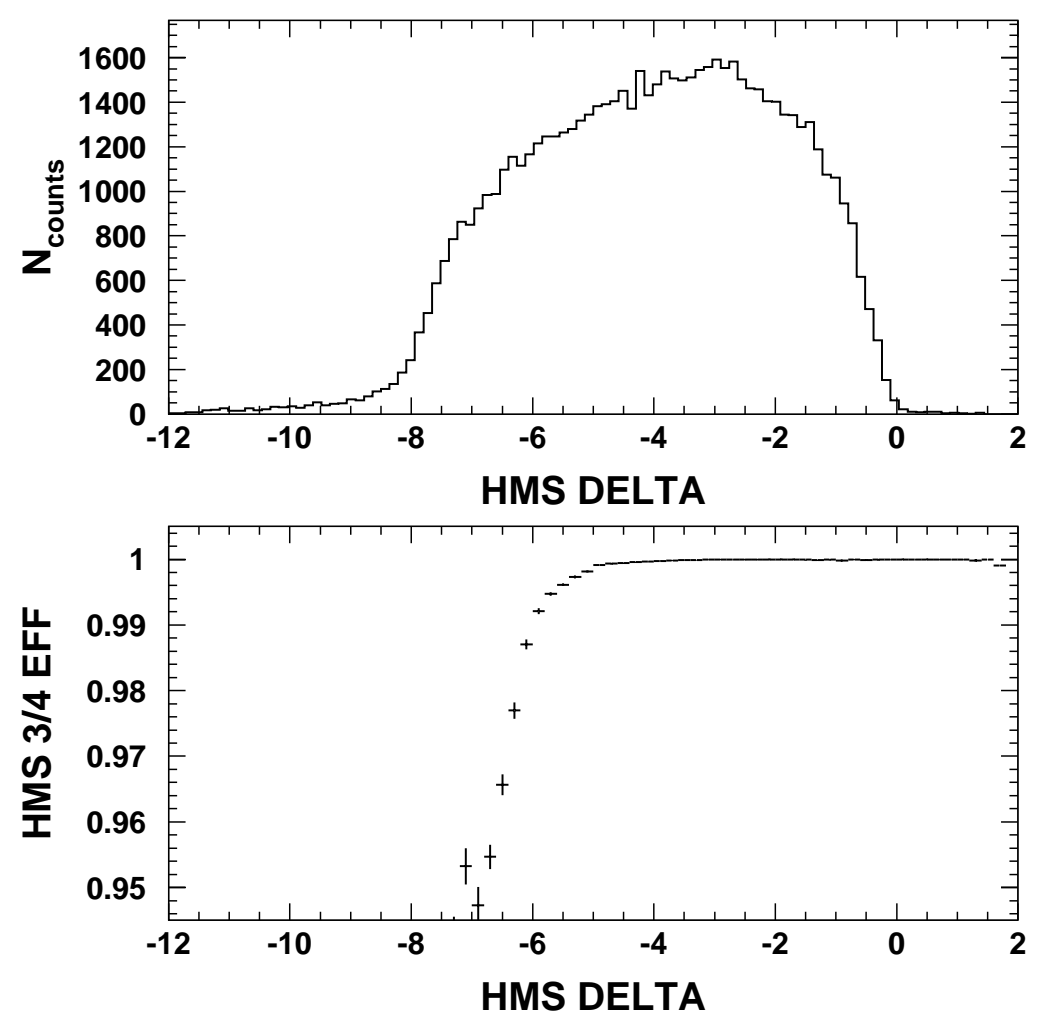

Figure 3.17: HMS trigger efficiency from elastic $\left(e, e^{\prime}\right)$ data. The upper panel shows the acceptance for electron kinematics with central $\delta$ centered at $-4 \%$. The lower panel shows the predicted 3/4 trigger efficiency using the algorithm described in the text. At negative $\delta$ the data indicate a significant ineffciency which may be attributed to scintillator paddle performance. This inefficiency is of no significant concern in the $F_{\pi}$-2 data analysis, since the HMS acceptance is limited from $-5 \%$ to $+8 \%$.

additional requirement on the particle type eliminates the bias introduced by the presence of other particle types in the acceptance with intrinsic lower efficiency (e.g. slow protons at positive polarity). In the HMS, particle identification to select pions in the calculation of the tracking efficiency was implemented in terms of cuts on the aerogel and gas Cerenkov. In the SOS electrons were selected using cuts on both the gas cerenkov and the calorimeter. The resulting tracking efficiencies in HMS and SOS are on the order of $97 \%$ and $99 \%$ respectively. 


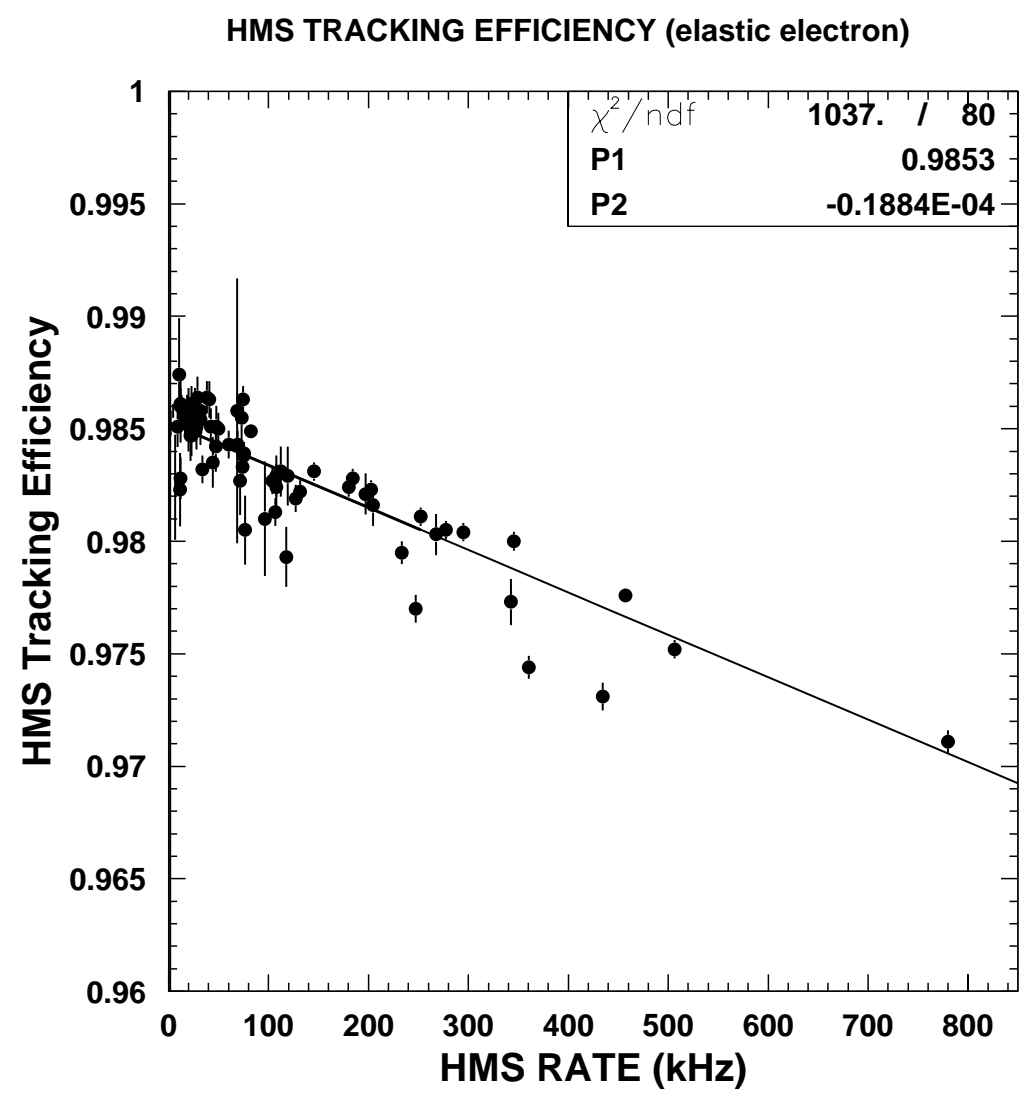

Figure 3.18: $F_{\pi}$-2 tracking efficiency versus rate.

Failure to produce a valid track can be attributed to insufficient or excessive information from the drift chambers. Drift chamber inefficiencies arise largely from the number of hits found exceeding the number of hits allowed in the chamber. The number of allowed hits per chamber is limited to 25 hits in both HMS and SOS and was chosen to keep tracking efficiencies high while simultaneously optimizing data processing time. Untracked events can also arise from insufficient hits in the drift chamber. The tracking routine requires hits in at least five planes in each chamber before attempting to recontruct a track for an event.

At high rates there is a nonzero probability for more than one particle passing 
through the chambers within the $\approx 250$ ns $\mathrm{TDC}$ window used for $\mathrm{F}_{\pi}-2$ analysis. The tracking algorithm only reconstructs a single good track for each event, but the loss of an "extra" good track is taken into account by one of the deadtime corrections depending on the order and particle type encountered in the chambers. The electronic deadtime corrects for additional tracks which are blocked from producing another trigger. In addition, the computer deadtime corrects for tracks lost passing through the drift chamber after the track that gave a valid trigger and is read out by the data acquisition. In coincidence configuration a coincidence lost in the event that a single trigger is not recorded because it is prescaled away is still taken into account by the coincidence blocking correction. However, even after correcting for various deadtimes the efficiency for reconstruction of one good track for a given trigger remains dependent on the presence of multiple tracks in the drift chambers.

To test the tracking efficiency calculation including multiple tracks a simple model was introduced in which the tracking efficiency is calculated from the individual single and multiple track efficiencies weighted by their contribution to the data sample. It should be noted that the choice of detected particles in all tracking efficiency studies is restricted to electrons. Taking into account multiple track events, the total tracking efficiency can be estimated from the simple formula,

$$
\epsilon_{t r}=P_{1} \cdot \epsilon_{1}+P_{2} \cdot \epsilon_{2}
$$

where $\epsilon_{t r}$ is the total HMS tracking efficiency, $P_{1}$ is the probability for a single 

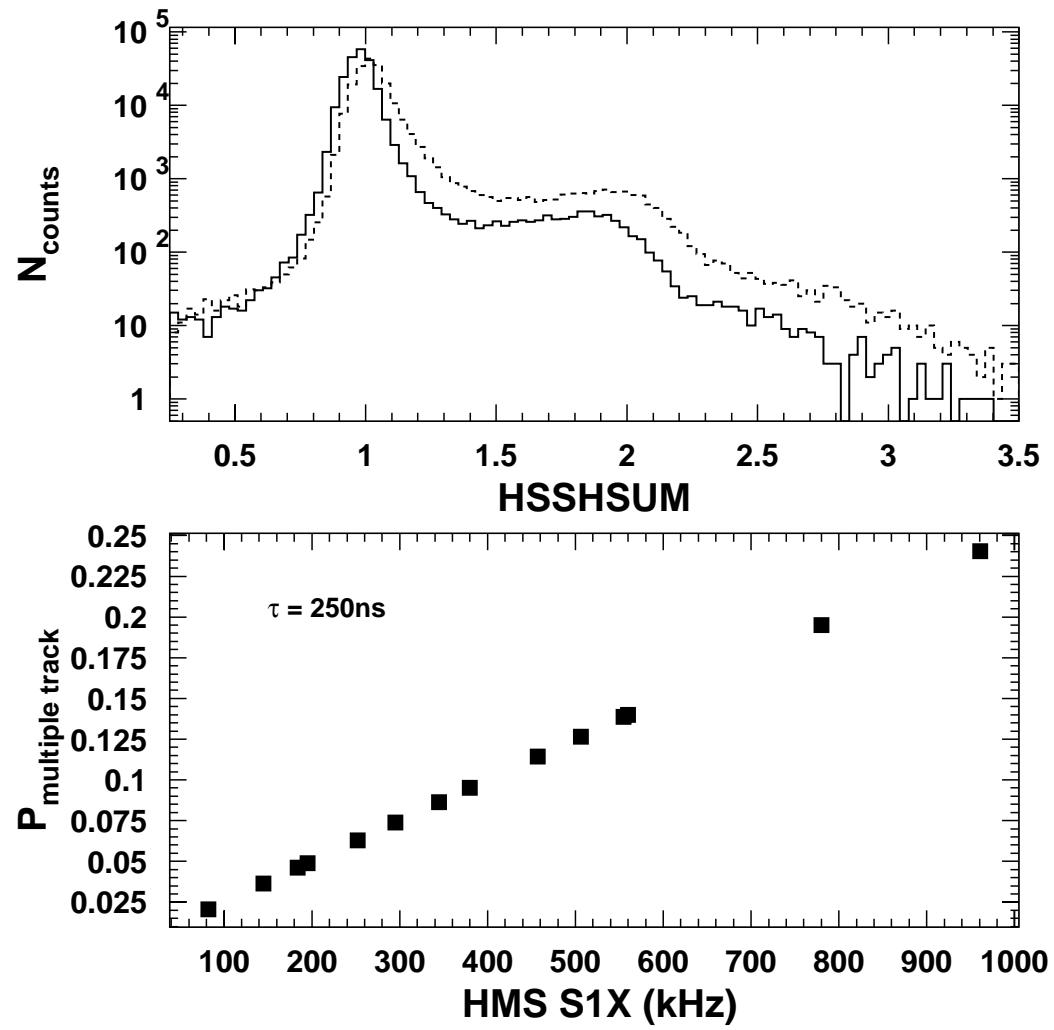

Figure 3.19: Multiple track contribution to electron data. The upper panel shows a distribution of the total energy deposited in the calorimeter. Nominally, electrons deposit all energy and appear at unity (normalized). Two electron clearly contribute in the region above 1.0 and the contribution changes as a function of rate. The solid line shows data taken at a rate of $400 \mathrm{KHz}$ and the dashed line indicates data taken at a rate of $700 \mathrm{KHz}$. The lower panel shows the multiple track background as a function of rate calculated from equation 3.16 .

electron passing through the chambers, $P_{2}$ is the probability for multiple electrons traversing the drift chambers within $250 \mathrm{~ns}$ and $\epsilon_{1}$ and $\epsilon_{2}$ denote the tracking efficiency for single electrons events and multiple electrons events respectively. The efficiencies, $\epsilon_{1}$ and $\epsilon_{2}$, are assumed to be rate independent and the origin of the rate dependence is assumed to be attributable solely to the probability of multiple track pile-up within the allowed $250 \mathrm{~ns}$ drift chamber gate width. Using Poisson statis- 
tics, the probability for arbitrary multiple track events through the drift chambers independent of particle identification can be expressed as,

$$
P_{2}=1-e^{(-R \tau)} \approx R \tau
$$

where $R$ is the drift chamber rate and $\tau$ denotes the drift chamber gate width used in the analysis. Substituting equation 3.16 into equation 3.15 and setting $P_{1}=1$ $P_{2}$ allows for expressing the total tracking efficiency in terms of the multiple track probability and the single and multiple electron efficiencies only.

The multiple track efficiency, $\epsilon_{2}$, can be determined from data by isolating multiple track events via the energy deposition in the calorimeter and calculating the tracking efficiency for these events. This efficiency is $70 \%$ and rate independent. The single track efficiency is obtained in a similar fashion and is generally higher by more than $25 \%$ relative to the intrinsic multiple track efficiency $(\approx 98.5 \%)$. It should be noted that the rate in the first scintillator layer (S1X) was used to estimate the total particle rate in the drift chambers rather than the pretrigger rate. The resulting efficiency prediction agrees to within $\approx 1 \%$ with the efficiency calculation used in the analyzer. The discrepancy observed at high rates can be attributed to differences in drift chamber and scintillator rates (the geometry of S1X is slightly larger) used in the model prediction of the tracking efficiency.

Previously, the tracking efficiency calculated in the Hall C ENGINE included a bias towards single track events. In particular, including multiple track events that arrive within the allowed drift chamber gate eliminates an intrinsic biasing of the 


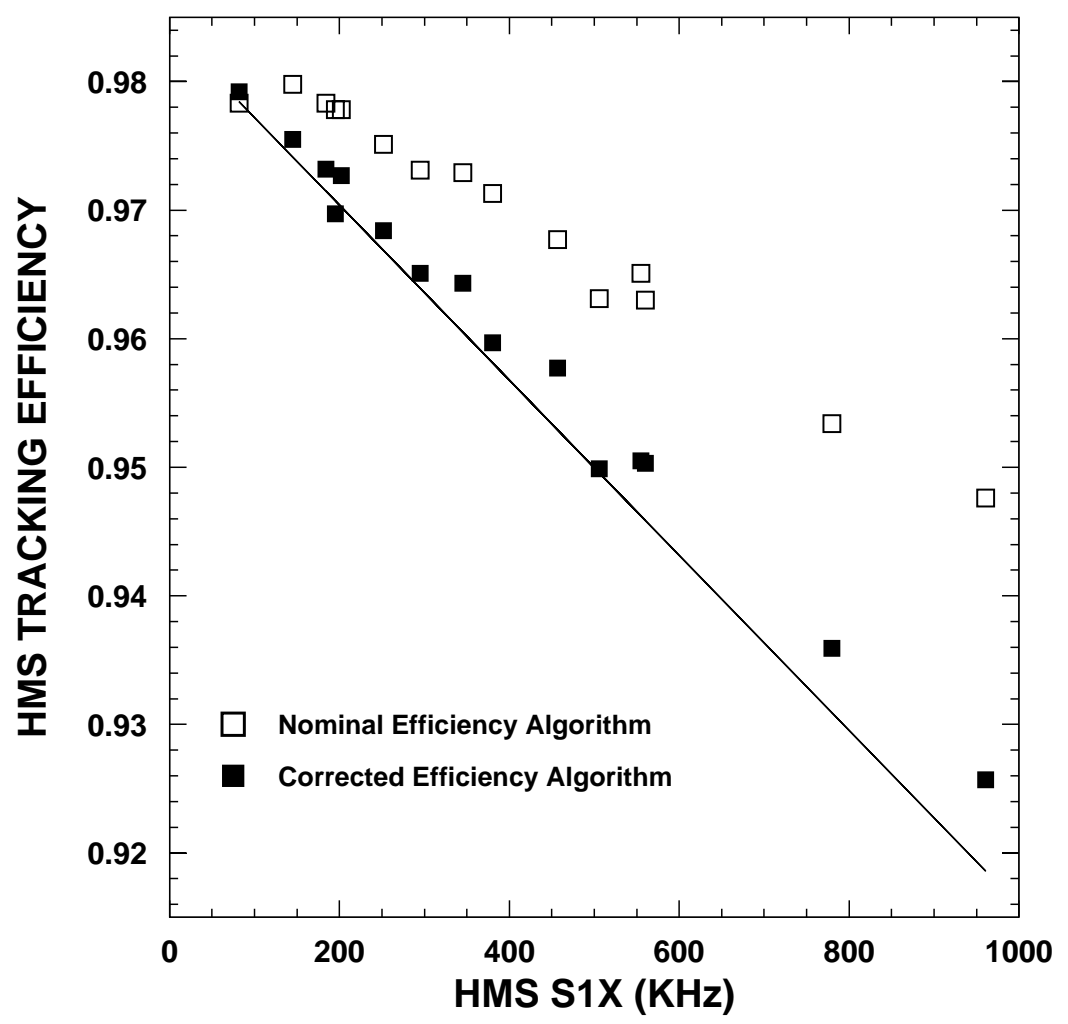

Figure 3.20: HMS tracking efficiency for inelastic electron data taken during luminosity scans. The open symbols indicate the nominal tracking efficiency calculation neglecting multiple track contribution and the filled symbols represent the calculation correcting for multiple tracks. The solid line indicates the prediction of for the HMS tracking efficiency using equation 3.15.

tracking efficiency calculation towards single track events. The bias is introduced in software by dis-allowing events with signals from multiple, separated scintillator paddles in a single plane. Whereas this approach is desirable for a low signal to noise ratio in order to reduce chamber noise due to, for example, scraping in the magnetic elements, it results in an over-estimate of the tracking efficiency at high rates and high signal to noise ratio. The difference of including multiple track events in the tracking calculation is on the order of $1 \%$ at rates of $\approx 200 \mathrm{kHz}$ and $5 \%$ at $1 \mathrm{MHz}$ 
(see Figure 3.20), thus a significant effect. In the $\mathrm{F}_{\pi^{-}}-2$ analysis the unbiased tracking efficiency including multiple hit events was used and applied on a run-by-run basis.

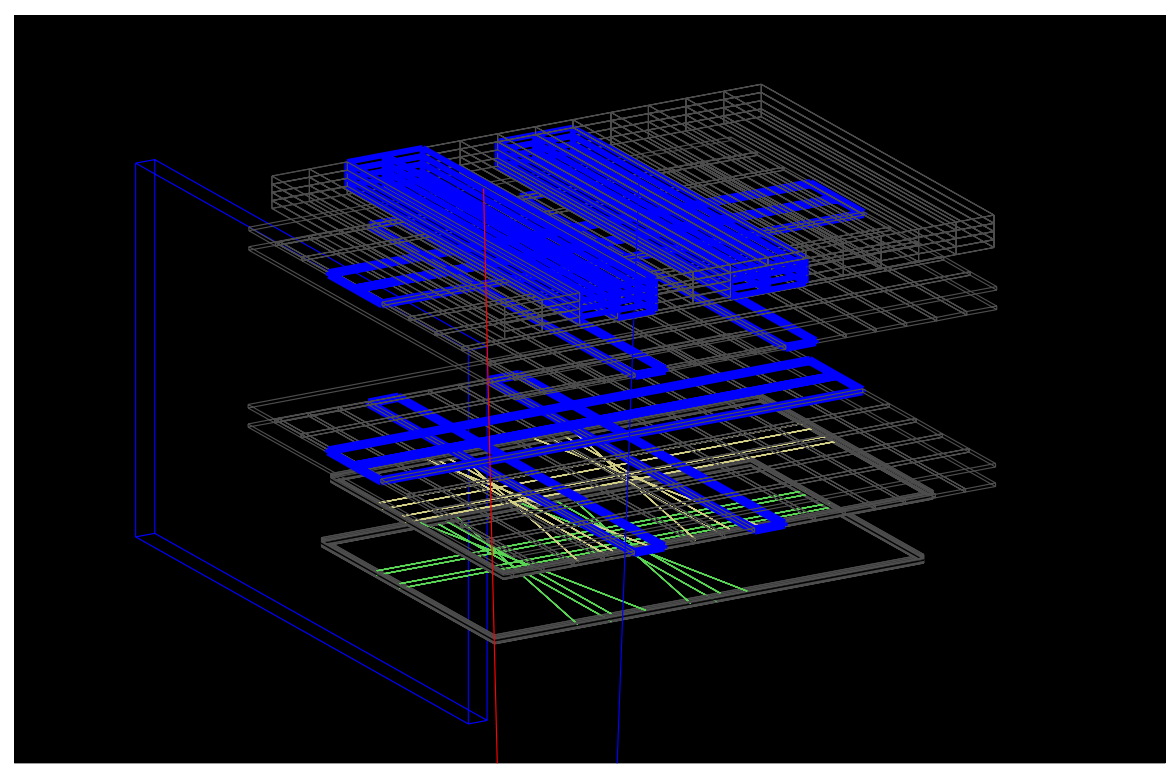

Figure 3.21: Schematic of the HMS detectors in one event display mode. Shown is an electron trigger for which multiple tracks were found. The red line indicates the track selected by the tracking algorithm and the remaining cluster of events are failed events.

\subsubsection{Computer and Electronic Deadtimes}

The processing of an event via electronic modules or computers requires a finite time during which particular components of the data acquisition are not able to accept any events. Events not recorded during this "deadtime" have to be corrected for in the data analysis. The types of deadtime encountered in a data acquisition system can be categorized into non-extendible and extendible deadtimes [91]. Whether or not a particular deadtime belongs to one of these categories depends on the behaviour of the corresponding hardware or software module regarding additional events and prolong the output gate accordingly while processing the previous 
event. Whereas extendible components are able to accept additional input events while processing the previous event, non-extendible components disregard any events until the previous event is processed.

The probability for detecting $n$ events in a time interval $t$ for a given event rate $R$ can be written,

$$
P(n)=\frac{(R t)^{n} e^{-R t}}{n !}
$$

where the mean of the Poisson distribution, $R$, corresponds to the mean number of events occurring in time $t$. Therefore, the number of events lost during a time interval $\tau$ in which the data acquisition is dead is simply $R \tau$. In the case of nonextendible deadtime the output gate is not extended to accept additional events and the live time can be expressed in terms of the ratio of the number of events detected and the true number of events which occurred in time $\tau$,

$$
(L . T .)_{n o n-e x t e n d i b l e}=\frac{1}{1+R \tau} \text {. }
$$

The computer deadtime is an example of a non-extendible deadtime. While the Trigger Supervisor is processing an event it sends a signal to the 8LM that inhibits all output from this module until the Trigger Supervisor is able to accept new events.

Given a particular trigger type the computer deadtime can be directly measured from the number of triggers and pretriggers as registered by the scalers. Once a pretrigger is formed, the pretrigger module forwards the signal to the scalers and 


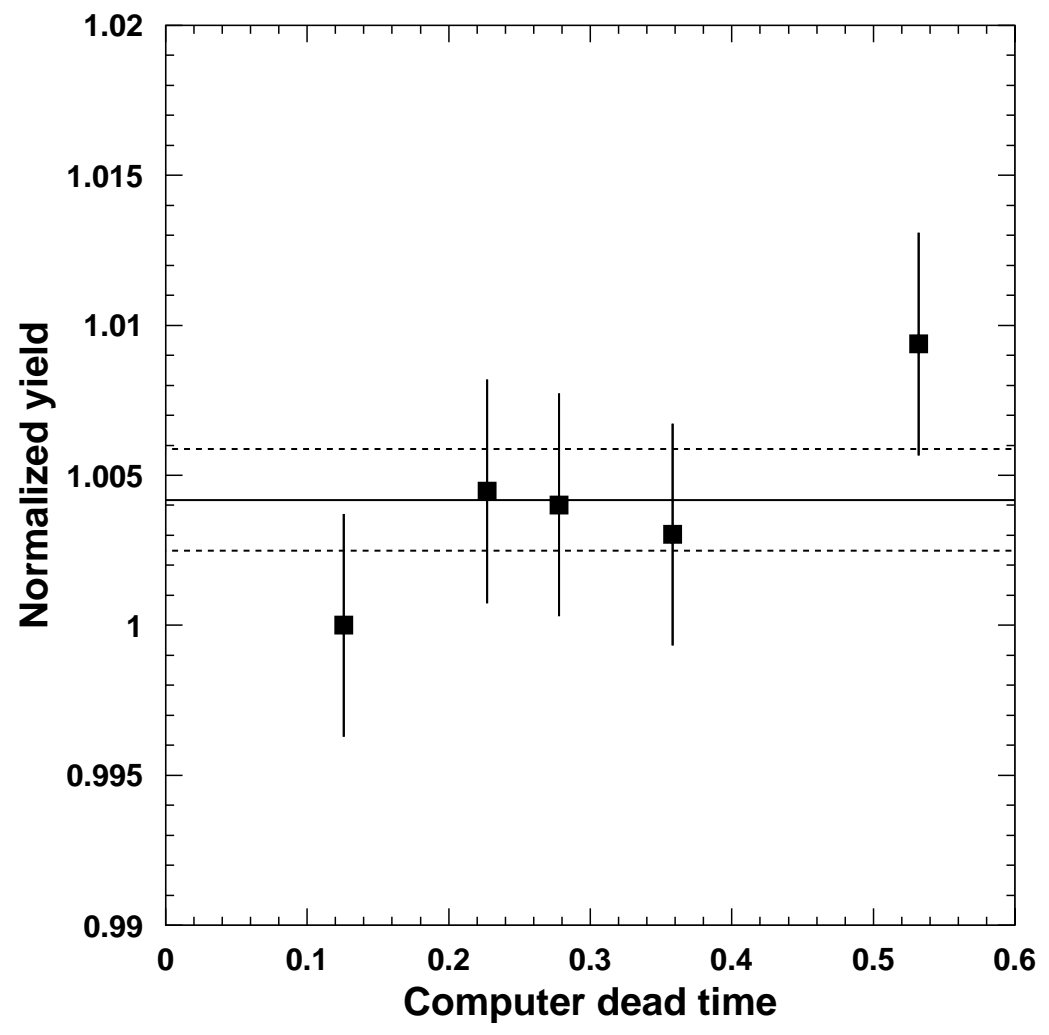

Figure 3.22: Efficiency corrected and charge normalized yields for data runs taken at constant current, but at varying computer deadtime. The error bars shown are statistical only. The solid and dashed lines indicate the average yield and the statistical deviation from it. While the computer dead time correction varies by $40 \%$ between runs, the yields agree to $0.2 \%$.

also to the $8 \mathrm{LM}$ module. If the $8 \mathrm{LM}$ is not inhibited by the Trigger Supervisor it creates a trigger signal and sends it on to the Trigger Supervisor. Note that the pretrigger signal from the $8 \mathrm{LM}$ is never inhibited. The trigger signal from the $8 \mathrm{LM}$ is processed at the Trigger Supervisor where the event is either prescaled (in the case of singles events) or read out to the ADC and TDC scalers. While the Trigger Supervisor is processing an event it sends the "TS BUSY" signal to the 8LM which suppresses any further output from the module until the Trigger Supervisor is ready 
to accept new events. The number of events missed due to the computer not being able to accept additional events can then be expressed as the ratio of the number of triggers put out by the 8LM (not inhibited) and the number of pretriggers.

$$
\text { Computer Livetime }=\frac{N_{\text {Trigger }}}{N_{\text {Pretrigger }}} .
$$

Both HMS and SOS scaler banks record the number of pretriggers. However, in the analysis, the SOS scalers are used due to erratic behavior noticed in the number of events recorded by the HMS pretrigger scaler. While a comparison of adjacent scaler readouts suggests a difference of less than 1\%, the Analyzer software interprets this change as a rollover of the scalers and adds a large number to the scaler which results in a large deadtime. The origin of the discrepancy between scaler readouts was attributed to a missing bit in the electronic modules. An indication of the effective gate width, which represents the average time to process an event, can be visualized in terms of the measured livetime as a function of the total rate of events at the Trigger Supervisor. Deviations from the average processing time may be due to a variation in the ratio of singles and coincidence rates. Generally both HMS and SOS singles can be processed faster with respect to coincidence events.

The measurement of the computer live time becomes extremely sensitive to the experimental conditions at high rates. For example, the choice of computer readout method at high rates can result in deviations from the theoretical prediction. In addition, the two single arm computer deadtimes during the measurement are generally not identical whenever high singles rates are accepted in one spectrometer 
while the rates into the other spectrometer were low. It should be noted that the trigger from the high rate spectrometer, in this case, is generally heavily prescaled. The difference in deadtimes can then be explained with deadtime correlations between the two spectrometers. In the case of the high event rate spectrometer the inputs to the Trigger Supervisor arrive in relatively regular intervals mimicking a clocklike behaviour. As a result the computer deadtime is largely governed by the other spectrometer. In contrast, the deadtime of the low rate event spectrometer is determined by its own rate and in addition by any contribution from the high rate spectrometer. The difference in the resulting deadtime is relatively small $(<1 \%)$ while the typical size of the deadtime correction is on the order of $10 \%$.

High rate data taking also affects the scaler readout. Although the scalers are read out at regular 2 second time intervals there is a nonzero probability that scaler readout becomes out of sync with actual data taking. For example, if the data acquisition is paused during data taking the scalers continue to increment, but are not read out. In particular, the charge scaler continues to increment even if no data are taken. This can result in false values of the total charge for a particular measurement. To eliminate the "out-of-sync" scalers the "syncfilter" option was introduced in software in 2003. Using a new set of variables and accounting for the timing between scaler readout, scalers not associated with actual data taking can be effectively excluded. Since the new set of variables is only used when the syncfilter option is enabled it is important to use the appropriate variables in the data analysis. $\mathrm{F}_{\pi}-2$ data were analyzed with the syncfilter option enabled.

The measurement of the computer live time at high rates can be tested using 
experimental data taken at fixed current and varying computer deadtimes. If the computer live time measurement is accurate the calculated charge normalized and computer livetime corrected yields are expected to be independent of the computer livetime. During $\mathrm{F}_{\pi}-2$ data were taken at a beam current of $80 \mu \mathrm{A}$ and deadtimes between $11 \%$ and $55 \%$ (changed by varying the prescale factor). The resulting normalized and corrected yields are shown in Figure 3.22 and confirm the hypothesis of no live time dependence. In the analysis the data are corrected using the experimentally measured computer live time on a run-by-run basis. The uncertainty in the computer live time is estimated from the distribution of yields about the mean value for computer deadtimes between 10 and $55 \%$. The resulting uncertainty is $0.2 \%$

Electronic deadtime arises when logic or discriminating modules ignore any further input while processing events. Since the electronics modules can have extendible or non-extendible output signals the calculation of the resulting dead time is not as clearly defined as in the case of the computer dead time.

An extendible module generates a $40 \mathrm{~ns}$ pulse for every event arriving. If a second event arrives a time $\Delta \tau$ after the first event and within the effective gate width, the total output signal from the module will be extended by $40+\Delta \tau$ ns. However, only one of the events will be recorded by the data acquisition even if this event does not correspond to the initial event. The probability for events occurring in time interval $\mathrm{t}$ scales with rate:

$$
P(t)=R e^{-R t} .
$$


The extendible livetime is then defined as the probability for no events occuring within time interval $\mathrm{t}(t>\tau)$,

$$
\text { Extendible L.T. }=\int_{\tau}^{\infty} R e^{-R t} d t=e^{-R \tau} .
$$

Since the effective gate width is very small ( $\approx 60 \mathrm{~ns})$, the small rates $R \tau<<1$ limit is always satisfied and equation 3.21 can be approximated by

$$
\text { Extendible L.T. } \approx 1-R \tau \text {. }
$$

This expression is true in the small $R \tau$ limit for both extendible and non-extendible modules, so it is not necessary to distinguish the two cases for determination of the electronic deadtime.

While the computer time can be directly measured the electronic deadtime is estimated from copies of the original pretrigger signal at varying limiting gate widths. This is done using the PRE50, PRE100, PRE150 and PRE200 scalers with corresponding gate widths of 40 ns, 100 ns, 150 ns and 200 ns (see Figure 2.16). Note that the gate width of the PRE50 scaler is reduced by 10 ns. This was designed as a check on the condition PRE50=PRETRIG, which should always be true since $40 \mathrm{~ns}<60 \mathrm{~ns}$. The true limiting gate width in the trigger logic corresponds to the width of the pretrigger output and is of width $\approx 60$ ns. Knowing the length of the individual PRExx gates and the limiting gate width of the pretrigger in the limit of small rates allows for the extrapolation to zero gate width and the calculation of 
the true number of events encountered. In particular, for a limiting gate width of $60 \mathrm{~ns}$ (dictated by the pretrigger) the true number of counts is given by

$$
N_{\text {True }}=N_{\text {measured }}+\left(\frac{N_{P R E 100}-N_{P R E 150}}{50 n s}\right) \cdot(60 n s) .
$$

and the electronic deadtime is

$$
\text { Electronic D.T. } \approx \frac{6}{5} \cdot\left(\frac{N_{P R E 100}-N_{P R E 150}}{N_{P R E 100}}\right)
$$

where the approximation $N_{P R E 100}=N_{T R U E}$ has been made. The electronic deadtime in the analysis is calculated from equation 3.24 for HMS and SOS scalers separately and is applied on a run by run basis. The rate dependence of the electronic livetime calculated for HMS and SOS is shown in Figure 3.23, where the parametrization is given by equation 3.22. The uncertainty in the correction is determined from the difference of the fitted limiting gate width, $\tau$, relative to its expected value of $60 \mathrm{~ns}$. This results in uncertainties of $6.5 \%$ and $22 \%$ of the size of the correction for HMS and SOS respectively. The typical size of the correction is then $3 \%$ for HMS and $0.4 \%$ for SOS which results in a combined uncertainty of $0.2 \%$. The origin of deviations of the fitted and expected gate widths may be attributable to uncertainties in estimating the true limiting gate width.

Determining the true limiting gate width was complicated by two factors during $\mathrm{F}_{\pi^{-}}$-2. Firstly, studies of the verification of the electronic deadtime revealed the necessity to assume a different limiting gate width for the HMS PRE200 scaler to 
obtain a consistent result for the electronic dead time. This behaviour was observed in previous experiments $[55,92,81]$ and was attributed to a missetting in the individual gate widths. However, the measured gate widths during $\mathrm{F}_{\pi}-2$ did not deviate significantly from the theoretical values. Offline studies of the expected gate widths using the measured number of counts from the PRE50, PRE100, PRE150 and PRE200 and PRETRIG scalers were in relatively good agreement with the expected gate widths for the two intermediate gate widths. In fact, the PRE200 signal could be shown to behave as expected when moved to another channel. In the $\mathrm{F}_{\pi^{-}}$2 analysis the PRE100 and PRE150 scalers were used in all calculations of the electronic deadtime.

An additional complication in diagnosing the proper degree of freedom to calculate the electronic dead time arises in the SOS due to the particle identification built in the trigger (ELREAL). In particular, calculating the electronic deadtime from equation 3.24 yields a result inconsistent with the pretrigger gate width while a similar calculation using data taken with an unbiased trigger agrees well with the pretrigger gate. Therefore, the true limiting gate width for the case of PID included in the trigger may be influenced by more than the pretrigger rate alone. The additional particle identification in the HMS (Čerenkov veto) does not have a noticeable effect on the HMS electronic dead time.

A comparable minor effect in the diagnosis of the HMS limiting gate widths was attributed to the erratic behaviour in one of the PRE scalers. In particular, the HMS PRE50 scaler always recorded more events than the PRETRIG scaler, which is logically not possible (see trigger logic diagram). A subsequent investiga- 
tion eliminated several sources of this behaviour. Cross talk between the electronic modules was ruled out by changing the signal cable lengths. Further tests on the electronic module configuration included: 1) using a passive fan-out of the PRETRIG signals instead of the nominal active fan-out 2) using a signal generator to study the input and output of the NIM-ECL converter. Both tests indicated no problems with the electronic modules. Additional tests with modified PRETRIG gate widths suggested a slight correlation between gate width and the high count rate in the PRE50 scaler. However, this study was done using cosmic data with intrinsic low rates so that the result of this analysis could not be considered as conclusive evidence. While the behaviour of the HMS PRE50 scaler is of concern in general, it is of no consequence in the data analysis, since it is not used in the calculation of the electronic deadtime and the effect was quite small.

\subsection{Coincidence Blocking}

The coincidence time between the spectrometers is used in the analysis to define good coincidence events. Such a coincidence event will normally be started at the TDC with a delayed HMS trigger (for HMS coincidence time as used in the analysis), and stopped by the SOS. However, due to interference between noncoincident and coincident events a fraction of events are recorded with a value of coincidence time outside the main timing window as defined by the pretrigger signal widths. These "coincidence blocking" events will be lost from the data due to the coincidence time cuts used in the analysis.

A typical raw (not corrected for pathlength) coincidence time spectrum is 

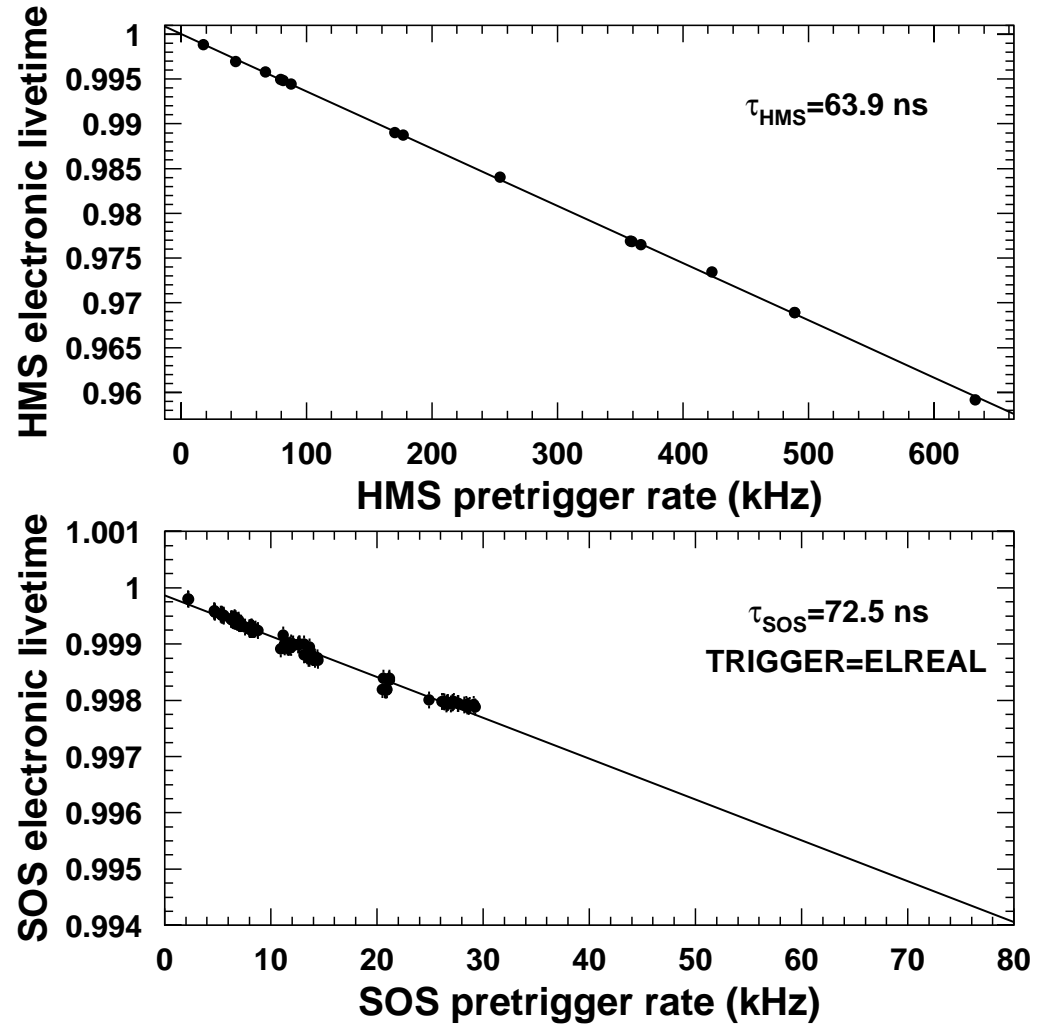

Figure 3.23: Electronic live time as a function of pretrigger rate into HMS and SOS. The data are fit to equation 3.22. The data shown are taken with no particle identification on the trigger level.

shown in Figure 3.24. The main coincidence time window corresponds to the region between 1130 to 2225 TDC channels. The conversion to nanoseconds is approximately TDC channels/10ns. The effective timing window width corresponds to the sum of HMS and SOS pretrigger gate widths $(60 \mathrm{~ns}+60 \mathrm{~ns}=120 \mathrm{~ns})$. The region to the left of the main timing window represents coincidence blocking due to early SOS singles triggers arriving before the coincidence SOS trigger. Thus the TDC is stopped too early resulting in a smaller value of the measured coincidence time. There are no early HMS events which can be atttributed to the particular setup of the trigger 


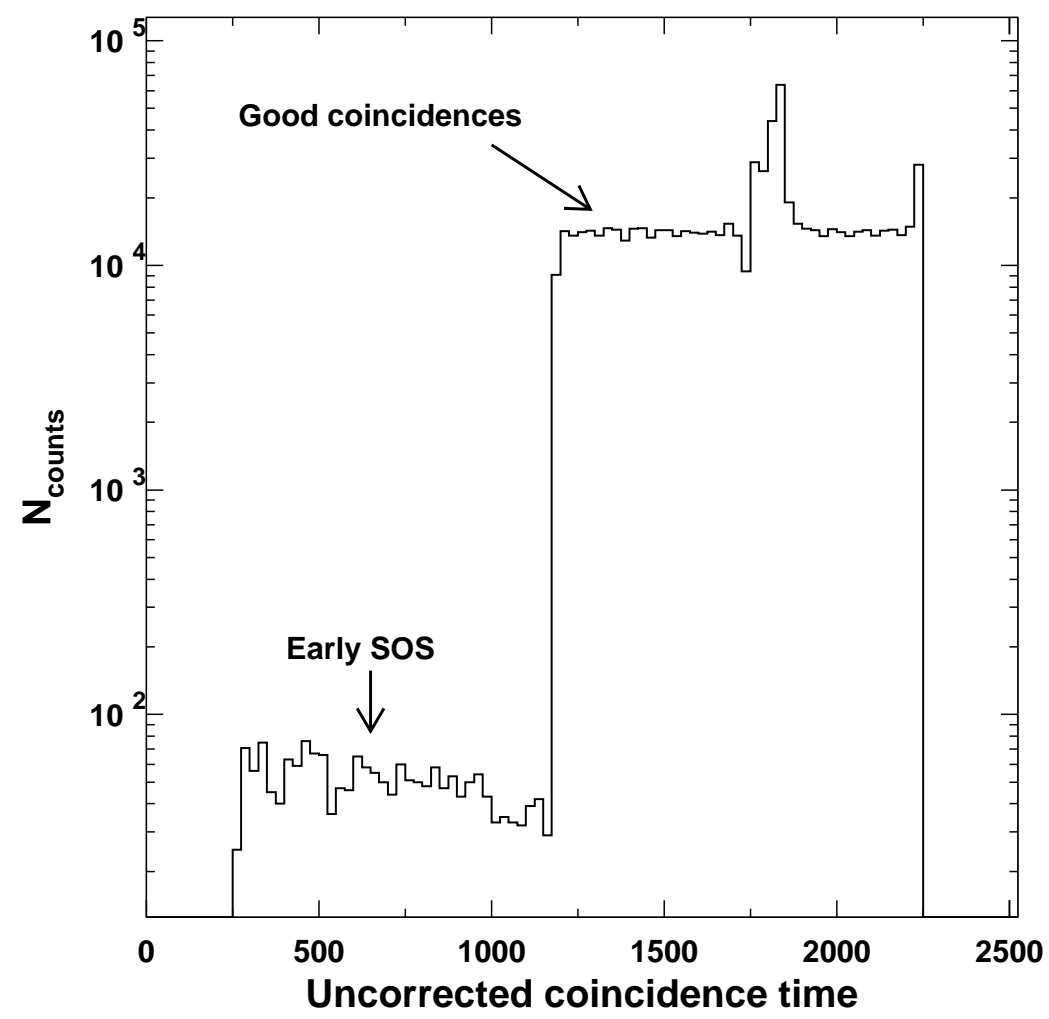

Figure 3.24: HMS Coincidence Time Spectrum. The main coincidence time window is centered around 1700 TDC channels, while the region to the left are early singles events in the SOS. Due to the specific coincidence time trigger conditions no early events from HMS singles are present. The conversion to nanoseconds is 0.1 TDC channels/ns.

during $\mathrm{F}_{\pi}$-2. In the absence of coincidence blocking the events in the region to the left of the main coincidence time window would have been good coincident events and all events lost in the timing cuts have to be corrected for.

The coincidence blocking correction can be estimated from the rate dependence of the number of blocked events similar to the deadtime correction estimations discussed in section 3.5.3. In particular, the comparison of the number of events 
outside of the main coincidence time window and the total number of events yields,

$$
\text { Coinblock }=\frac{N_{b l k}}{N_{t o t}}
$$

where $N_{b l k}$ denotes the number of "early" SOS events in the measured coincidence time spectrum and $N_{\text {tot }}$ is the total number of events independent of the coincidence time. The coincidence blocking correction was then parametrized in terms of an exponential function in the limit of large rates from

$$
\text { SOS Coinblock }=\left(1-R_{S O S} * \tau_{S O S}\right),
$$

where $R_{S O S}$ denotes the event rate into the SOS given by the pretrigger rate and $\tau$ denotes the value from the linear fit as shown in Figure 3.25. The uncertainty in the coincidence blocking correction is estimated from the difference in the measured and calculated values given by equations 3.25 and 3.26 respectively. The total uncertainty of the correction is on the order of $0.1 \%$.

\section{7 Čerenkov Blocking}

The HMS gas Čerenkov is used for electron rejection at negative polarity. The effective time window is given by the Čerenkov ADC and is $\approx 100 \mathrm{~ns}$ long. Events identified as electrons by the ADC appear at $\approx 50$ ns in the typical Cerenkov TDC spectrum (see Figure 3.26). Therefore, if a particle passes through the detector after the first particle, but within the effective ADC gate width, the second signal will 


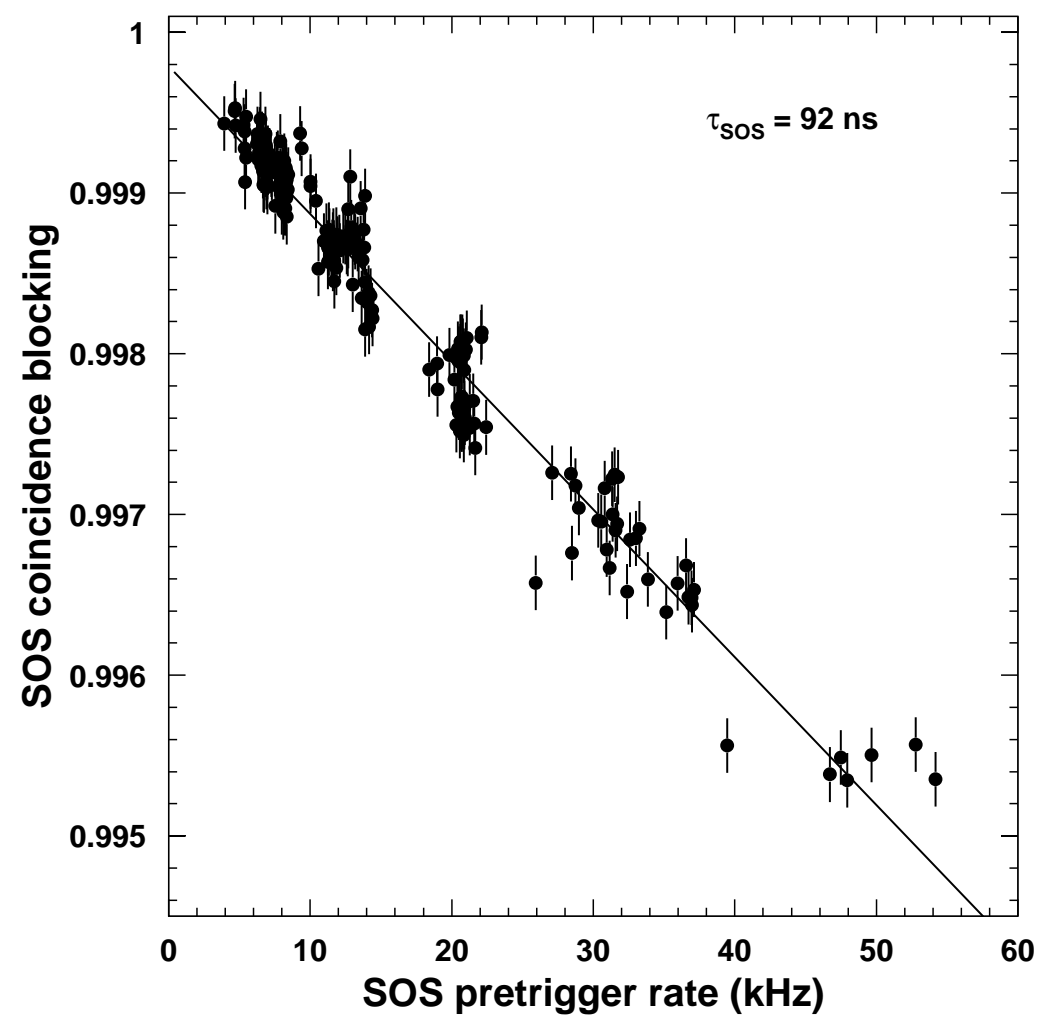

Figure 3.25: Measured SOS coincidence blocking correction due to SOS early singles as a function of pretrigger rate. The curves shown are the fit results to the data from equation 3.26 with the value of $\tau$.

also be read out in the ADC even if it is not associated with the original trigger. In particular, if an electron passes through the gas Čerenkov within about 90 ns after a pion, the pion event will be identified as an electron and effectively eliminated by analysis cuts applied.

A typical HMS Čerenkov TDC spectrum for a photoelectron requirement of $N_{\text {photoelctrons }}=0.5$ is shown in Figure 3.27. The main peak corresponds to true electron events as identified by the Cerenkov ADC. Events not associated with the original trigger appear as additional events to the left and right of the main electron 


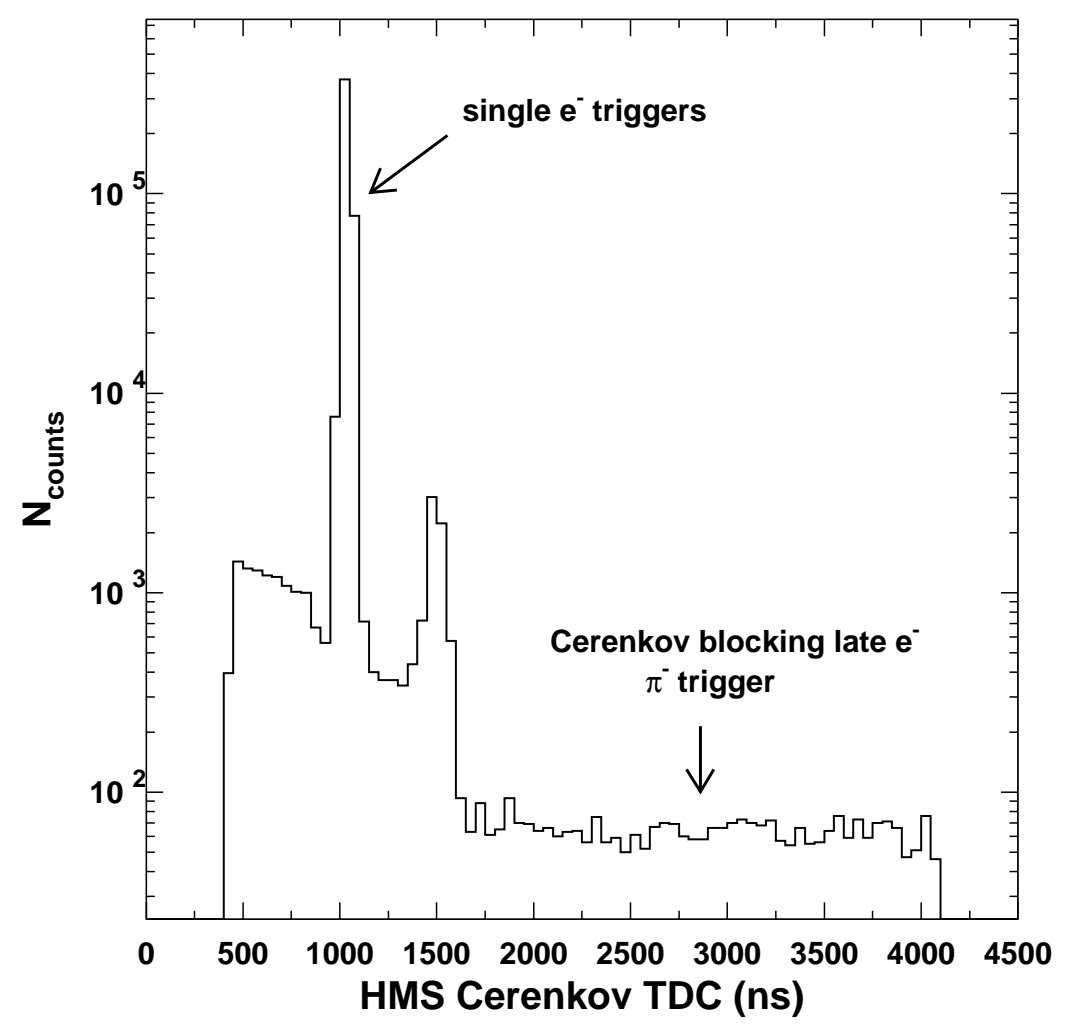

Figure 3.26: HMS Čerenkov Blocking TDC spectrum for electron events as identified by the HMS Čerenkov ADC. The central peak corresponds to signal from the electrons that result in the trigger. The second peak is due to a second electron arriving within the timing window. The backgrounds to the left and right of the two peaks are due to earlier and later electrons.

peak in the TDC spectrum. In particular, events to the left of the peak correspond to electrons passing through the detector before the electron associated with the trigger and events to the right of the main electron peak correspond to electrons traversing the detector after the original trigger electron. For the Čerenkov blocking correction only the region to the right of the main peak is of interest, since early electrons result in coincidence blocking of the pion signal, which is addressed in the coincidence time blocking correction described in section 3.6. 


\begin{tabular}{|l|c|c|c|}
\hline$Q^{2}(\mathrm{GeV} / \mathrm{c})^{2}$ & $\epsilon$ & $\mathrm{e}^{-}$rate $(\mathrm{KHz})$ & HMS C-block $(\%)$ \\
\hline 2.45 & 0.27 & 536 & 8.36 \\
& 0.54 & 311 & 4.85 \\
\hline
\end{tabular}

Table 3.4: HMS Čerenkov blocking correction at high and low $\epsilon$. The values shown are for representative runs at each setting.

The Čerenkov blocking correction can be estimated from the electron rate into the spectrometer and the effective Čerenkov TDC gate width $(\approx 100 \mathrm{~ns})$,

$$
\text { HMS Cer Block }=\left(1-R_{e} * \tau_{\text {Cer }}\right)
$$

where $R_{e}$ denotes the electron rate into the spectrometer as measured by the ELCLEAN scaler and $\tau=100 \mathrm{~ns}$ is the effective Cerenkov gate width. The overall Čerenkov blocking depends only on the incident electron rate and does not depend strongly on variations in run to run characteristics. Therefore it is sufficient to determine the size of the correction for given kinematics from a small sample of measurements. During $\mathrm{F}_{\pi}-2$ the Cerenkov blocking in the HMS at negative polarity was measured at the beginning of each kinematic setting with $3 / 4$ SCIN singles trigger. The singles trigger configuration was chosen to avoid biasing the sample of particles towards pions (as would be the case if coincidence data were used). The size of the correction for the highest rate measurements taken at each kinematic setting is shown in table 3.4 .

The fraction of pions lost in Čerenkov blocking is given by the ratio of experimental pion yield with and without Čerenkov cut: where both yields are defined as the number of good events normalized by charge and corrected for efficiencies. 


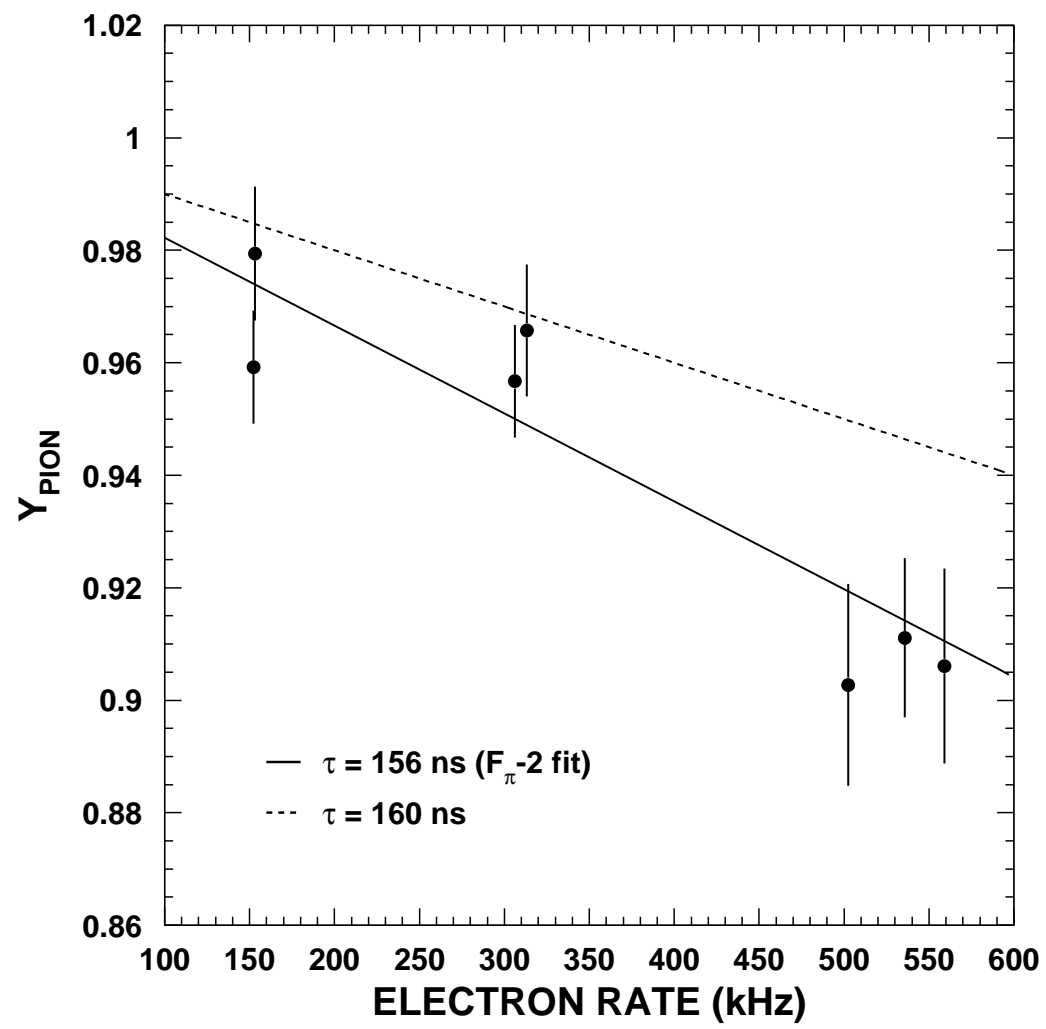

Figure 3.27: Čerenkov blocking as a function of electron rate in the HMS. The solid line is a fit to equation 3.2\%. The fit value for $\tau$ is larger than the measured Čerenkov $T D C$ gate width $(\approx 100 \mathrm{~ns}$, dashed line). This discrepancy may be attributed to the width of the Črenkov signal. While the ADC gate is fixed, the Čerenkov signal itself has some width and the overlap determines an effective gate width.

Good events are selected with loose cuts placed on the HMS acceptance. An additional requirement was placed on the HMS Čerenkov for particle identification $\left(N_{\text {photoelectrons }}>2\right)$.

The uncertainty in the HMS Čerenkov blocking correction is largely attributed to the uncertainty in the Čerenkov timing window. In particular the effective cerenkov gate width fit from equation 3.27 appears to be larger than the measured ADC gate $(\approx 100 \mathrm{~ns})$. This effect may be explained in terms of intrinsic widths of 
the signals. While the ADC gate is fixed, the Čerenkov signal itself has some width and the overlap determines an effective gate width.

The size of the Čerenkov blocking correction from HMS singles analysis is on the order of $8 \%$ at a beam current of $35 \mu \mathrm{A}$. A comparison of the measured effective Čerenkov gate for singles and coincident events with the same cerenkov cut suggests that the coincidence correction can be determined from the corrections found from the singles analysis.

\subsection{Pion Absorption in the HMS}

Some pions are lost due to nuclear interactions in the materials that the particles pass through on their way from the target to the HMS detector hut. Pions lost in hadronic interactions are largely due to absorption and large angle scattering resulting in pions that do not strike all detectors required to form a trigger.

The transmission of pions through the spectrometer is defined as the fraction of pions that do not interact with any of the materials,

$$
T=e^{-\Sigma \lambda_{i} t_{i}}
$$

where $t_{i}$ and $\lambda_{i}$ denote the thickness and the interaction length of the $i^{\text {th }}$ material encountered in the particles path. The interaction length is defined as:

$$
\lambda=\rho N_{A} \frac{\sigma_{A}}{A}
$$


where $\rho$ is the material density, $N_{A}$ is Avogadro's number, $A$ is the atomic mass number and $\sigma_{A}$ denotes the relevant pion cross section for a material of atomic mass A. The pion transmission is then determined from equation 3.28 using the list of material information encountered in the HMS and the relevant pion-nucleon cross sections.

The calculation of the pion transmission through the materials is largely determined by the choice of pion-nucleus cross section used in the calculation. In particular, the total cross section, which is defined as the sum of all hadronic interactions, represents an underestimate of the transmission. This can be explained in terms of the contribution of the individual pieces to the effective loss of pions. Elastic scattering is peaked in the forward direction (small angles), so that a large fraction of the elastically scattered pions are expected to still produce a valid pion event. In addition, inelastic scattering does not necessarily correspond to an invalid trigger. On the other hand, a pion that is truly "absorbed" will clearly not result in a trigger. Therefore, the transmission is calculated from the reaction cross section which includes all hadronic interactions except for elastic scattering $\left(\sigma_{\text {reac }}=\right.$ $\left.\sigma_{\text {absorption }}+\sigma_{\text {inelastic }}\right)$. The reaction cross section is approximately the average of the total and absorption cross sections and the uncertainty on the transmission can be conservatively estimated from these two limiting cases.

The $A$-dependence of the reaction cross section for large A materials can be parametrized as follows,

$$
\sigma_{A}=\sigma_{0} A^{\alpha}
$$


where $\sigma_{0}$ and $\alpha$ are fit from experimental data. The reaction cross section is parametrized using both a fit to reaction cross section data from reference [93] for $\mathrm{T}_{\pi}>2000 \mathrm{MeV} / \mathrm{c}$ and data from table 3.5 in reference [55] for $T_{\pi}<2000 \mathrm{MeV} / \mathrm{c}$. For each material the cross section is calculated using the parametrization shown in Figure 3.28 and equation 3.30. The corresponding interaction length is calculated from equation 3.29 .
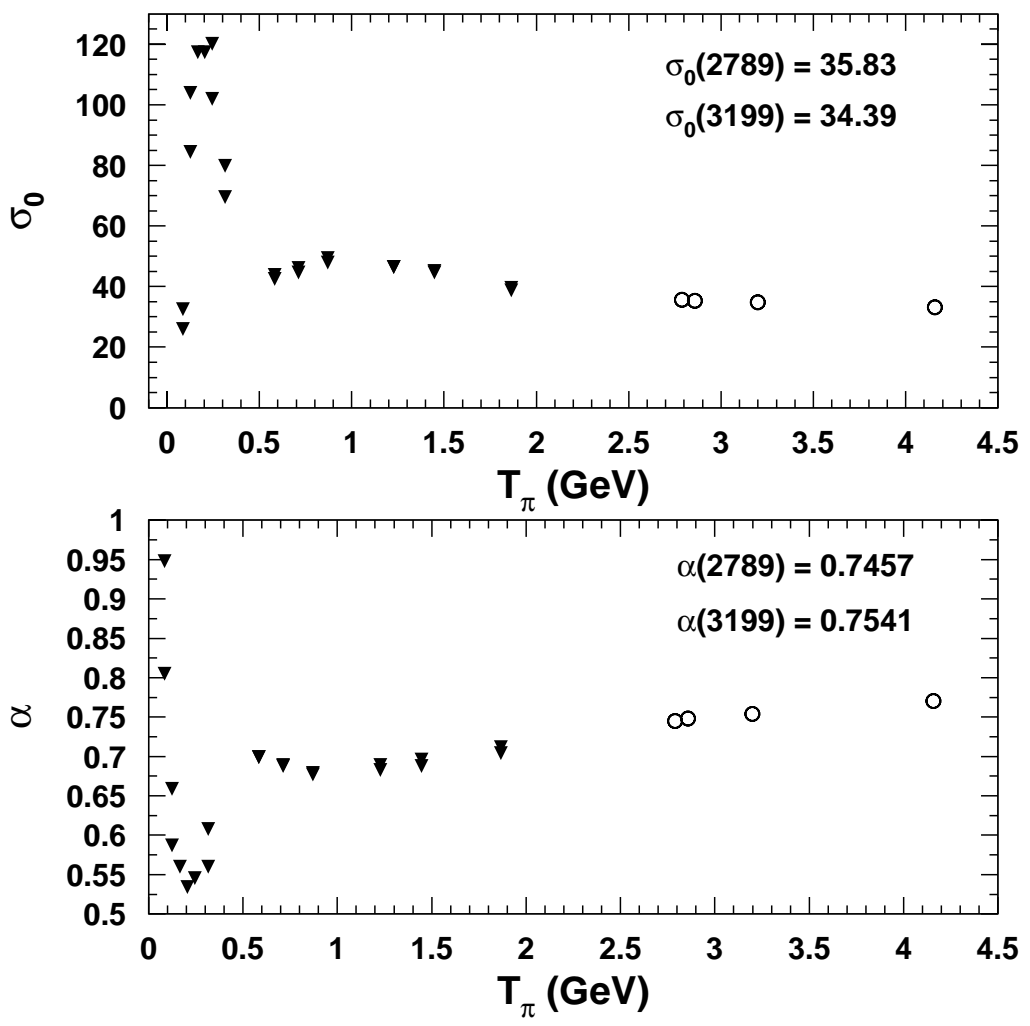

Figure 3.28: Parametrization of the $A$ dependence of the $\pi-A$ cross section. The data are from references [55, 93].

For the cryogenic targets $(A<3)$ the formalism described above does not apply and the reaction cross section is estimated from total cross section data. This approach is necessary due to the total lack of experimental data in terms of the 


\begin{tabular}{|l|c|c|c|c|}
\hline$P_{\pi}$ & Transmission & $\delta_{\text {target }}$ & $\delta_{\text {polarity }}$ & $\delta_{x \text { sec }}$ \\
\hline 2.931 & $\approx 0.95$ & $0.4 \%$ & $0.4 \%$ & $2.0 \%$ \\
3.336 & $\approx 0.95$ & $0.4 \%$ & $0.4 \%$ & $2.0 \%$ \\
\hline
\end{tabular}

Table 3.5: Pion absorption correction and associated uncertainties.

individual components of the total cross section. The lower limit on the transmission through the cryogenic targets can be estimated using the total cross section and is found to be relatively large $(>99.0 \%)$. The size of the total cross section is roughly twice the value of the reaction cross section as evidenced by experimental data, so that the true value of the transmission is expected to be less than $1 \%$. Given the size of the transmission through the target even a large uncertainty due to the cross section model does not significantly affect the result. The uncertainty between cryogenic targets is estimated from a comparison of the transmission calculated from total cross section and is determined to be $0.4 \%$ for both $T_{\pi}=2789 \mathrm{MeV} / \mathrm{c}$ and $T_{\pi}=3199 \mathrm{MeV} / \mathrm{c}$. The total uncertainty on the pion absorption correction can be estimated in a less conservative way from the measured proton absorption and the relative size of the proton and pion cross sections at the relevant kinematics. The resulting uncertainty at lab momenta of $3 \mathrm{GeV} / \mathrm{c}$ is $2 \%$. The calculated pion transmission and associated uncertainties are listed in Table 3.5. The transmission is calculated from equation 3.28 and using the list of materials in the HMS summarized in Table 3.6. It should be noted that only $1 / 4$ of the third scintillator is included in the calculation, because this distance is considered sufficient to produce a valid $3 / 4$ trigger, thus resulting in the pion to be detected. The pion transmission is applied as a global factor to the data on a run by run basis. Since the $\pi-A$ cross section 
depends only on the pion momentum, which remains fixed for both $\epsilon$ settings no $\epsilon$ dependent uncertainty is assigned. 


\begin{tabular}{|c|c|c|c|c|c|}
\hline Absorber & Material & $\begin{array}{c}\text { thickness } \\
(\mathrm{cm})\end{array}$ & $\begin{array}{l}\text { density } \\
\left(\mathrm{g} / \mathrm{cm}^{3}\right)\end{array}$ & $\begin{array}{c}X_{0} \\
\left(g / \mathrm{cm}^{2}\right)\end{array}$ & $\begin{array}{c}\left(X / X_{0}\right) \\
(\%)\end{array}$ \\
\hline Target & LH2 & 3.968 & 0.0723 & 61.28 & 0.468 \\
\hline Target Window & $\mathrm{Al}$ & 0.013 & 2.70 & 24.01 & 0.292 \\
\hline Chamber Window & $\mathrm{Al}$ & 0.0406 & 2.70 & 24.01 & 0.457 \\
\hline Chamber HMS Gap & Air & 15 & 0.00121 & 36.66 & 0.050 \\
\hline \multirow[t]{2}{*}{ HMS Entrance Window } & Kevlar & 0.0381 & 0.74 & 55.20 & 0.0511 \\
\hline & Mylar & 0.0127 & 1.39 & 39.95 & 0.0443 \\
\hline HMS Exit Window & Titanium & 0.0508 & 4.54 & 3.56 & 6.478 \\
\hline Dipole-DC Gap & Air & 35 & 0.00121 & 36.66 & 0.1155 \\
\hline HMS DC Windows & Mylar & $4(0.0025)$ & 1.39 & 39.95 & 0.0178 \\
\hline HMS DC Gas & $\mathrm{Ar} / \mathrm{C}_{6} \mathrm{H}_{6}$ & $12(1.8)$ & 0.00154 & 27.38 & 0.121 \\
\hline HMS DC sense wires & W & $12(5.89 \mathrm{E}-06)$ & 19.30 & 6.76 & 0.020 \\
\hline HMS DC field wires & $\mathrm{Be} / \mathrm{Cu}$ & $36(0.00018)$ & 5.40 & 38.88 & 0.090 \\
\hline HMS S1X & polystyrene & 1.067 & 1.03 & 43.80 & 2.51 \\
\hline HMS S1Y & polystyrene & 1.067 & 1.03 & 43.80 & 2.51 \\
\hline HMS Cer Windows & $\mathrm{Al}$ & $2(0.102)$ & 2.70 & 24.01 & 2.28 \\
\hline HMS Cer gas & $C_{4} F_{10}$ & 135 & 0.00246 & 23.70 & 1.40 \\
\hline HMS Cer mirror support & Rohacell & 1.8 & 0.05 & 40.88 & 0.220 \\
\hline Air gap DC-S2X & Air & 83.87 & 0.00121 & 36.66 & \\
\hline HMS S2X & polystyrene & 1.067 & 1.03 & 43.80 & 2.51 \\
\hline HMS S2Y & polystyrene & 1.067 & 1.03 & 43.80 & 2.51 \\
\hline Aerogel entrance & $\mathrm{Al}$ & 0.15 & 2.70 & 24.01 & 1.687 \\
\hline Aerogel & $\mathrm{SiO}_{2}$ & 9.0 & $0.04-0.06$ & 44.054 & 1.021 \\
\hline Aerogel air gap & Air & 16.0 & 0.00121 & 36.66 & 0.0528 \\
\hline Aerogel exit & $\mathrm{Al}$ & 0.1 & 2.70 & 24.01 & 1.1245 \\
\hline Chamber Window & $\mathrm{Al}$ & 0.0406 & 2.70 & 24.01 & 0.456 \\
\hline Chamber SOS Gap & Air & 15 & 0.00121 & 36.66 & 0.050 \\
\hline \multirow[t]{2}{*}{ SOS Entrance Window } & Kevlar & 0.0127 & 0.74 & 55.20 & 0.0170 \\
\hline & Mylar & 0.0076 & 1.39 & 39.95 & 0.0260 \\
\hline \multirow[t]{2}{*}{ SOS Exit Window } & Kevlar & 0.0381 & 0.74 & 55.20 & 0.051 \\
\hline & Mylar & 0.0127 & 1.39 & 39.95 & 0.044 \\
\hline Dipole-DC Gap & Air & 15 & 0.00121 & 36.66 & 0.050 \\
\hline SOS DC Windows & Mylar & $14(0.0127)$ & 1.39 & 39.95 & 0.062 \\
\hline SOS DC Gas & $\mathrm{Ar} / \mathrm{C}_{6} \mathrm{H}_{6}$ & $12(0.617)$ & 0.00154 & 27.38 & 0.044 \\
\hline SOS DC sense wires & W & $12(35.4 \mathrm{E}-06)$ & 19.30 & 6.76 & 0.121 \\
\hline SOS DC field wires & $\mathrm{Be} / \mathrm{Cu}$ & $36(0.00018)$ & 5.40 & 38.88 & \\
\hline SOS S1X & polystyrene & 1.040 & 1.03 & 43.80 & 2.44 \\
\hline SOS S1Y & polystyrene & 1.098 & 1.03 & 43.80 & 2.58 \\
\hline SOS Cer Windows & $\mathrm{Al}$ & $2(0.05)$ & 2.70 & 24.01 & 1.12 \\
\hline SOS Cer gas & $C_{4} F_{10}$ & 100 & 0.00510 & 23.70 & 2.152 \\
\hline SOS Cer mirror support & Rohacell & 1.8 & 0.05 & 40.88 & \\
\hline Air gap DC-S2Y & Air & & 0.00121 & 36.66 & \\
\hline SOS S2X & polystyrene & 1.040 & 1.03 & 43.80 & 2.44 \\
\hline SOS S2Y & polystyrene & 1.098 & 1.03 & 43.80 & 2.58 \\
\hline
\end{tabular}

151

Table 3.6: Materials in HMS and SOS contributing to absorption and multiple scattering as particles traverse the target and spectrometers. 
Chapter 4

Simulation

\subsection{Monte Carlo Overview}

The standard Monte Carlo program used in Hall C (SIMC) is based on the code SIMULATE, which was designed for SLAC experiment NE18 [102]. While the general structure has been kept, the code was modified to include the Hall $\mathrm{C}$ coordinate system and Monte Carlo simulations of current and future Hall C spectrometers. SIMC was designed as an aperture checking Monte Carlo and does not have the capability to simulate individual detector signals. However, SIMC includes all relevant details of the experimental conditions such as the rastering and energy spread of the electron beam, radiative processes, hadron decay and finite track resolution to ensure a realistic simulation of experimental data. SIMC can be used in both polarized and unpolarized target configurations. The reactions currently implemented in SIMC include $\left(e, e^{\prime} p\right)$ type reactions and meson electroproduction for both exclusive and semi-inclusive channels.

For each event the Monte Carlo generates both the initial coordinates of the interaction vertex $(x, y, z)$ and kinematic properties such as direction and momentum for the particles of interest. The starting values for the generation limits in angle and momentum are fixed by the input to the simulation and typically chosen to exceed the physical acceptance of the spectrometers. If the kinematics of an event are physically allowed, the outgoing event is radiated and followed on the trip through the target taking into account energy loss and multiple scattering. Note 
that all angles are generated in the spectrometer coordinate system. After the event generation is complete, the events are sent to the single arm spectrometer modules, which simulate the magnetic optics inside the Hall $\mathrm{C}$ spectrometers and propagate the particle through apertures and magnets into the spectrometer hut. Inside each spectrometer hut the particle trajectory is tested at each detector aperture. Events that clear all apertures and cross the minimum number of detectors in the huts are considered to produce a valid trigger. Particle trajectories are reconstructed to the target if a valid trigger was generated. Since only detector apertures are simulated, no inefficiencies are assigned in the event simulation. However, each event is weighted by the relevant model cross section corrected for radiative processes, a luminosity factor, and a Jacobian taking into account the transformation between spectrometer and physics coordinates.

\subsection{Event Generation}

The pion electroproduction cross section from hydrogen is determined in terms of five degrees of freedom which have to be simulated. The Monte Carlo generates the spectrometer in- and out of plane angles for electron and hadron and the electron energy in a uniform distribution. The limits of the distributions are not strictly fixed by the spectrometer acceptance, thus allowing for multiple scattering and energy loss. Given the angles and final electron energy the momentum of the hadron can be calculated from energy-momentum conservation.

The simulation of pion electroproduction from a nuclear target is complicated by the extra degree of freedom introduced by the relative momentum between the 
struck nucleon and the nuclear medium. For example, the pion electroproduction cross section from deuterium depends now on six independent variables so that the pion momentum is not determined from the five quantities described above. However, assuming quasifree electroproduction from a single nucleon one can generate the nucleon momentum based on the Fourier transform of the struck nucleon wavefunction. A detailed description of pion electroproduction from nuclear targets can be found in Chapter 4 of reference [55].

With all degrees of freedom specified the five fold pion electroproduction cross section can be calculated. The model pion production cross section for a simulated event in SIMC is written in terms of the center of mass photoproduction cross section $\left(d \sigma / d \Omega_{\pi}^{*}\right)$ and the virtual photon flux factor, $\Gamma$. However, the generated quantities in SIMC are generated in the laboratory system and to calculate the appropriate cross section weight the virtual photon cross section has to be expressed in the lab frame. The transformation in the case of hydrogen can be written in terms of the invariant cross section $d^{2} \sigma /(d t d \phi)$ :

$$
\frac{d^{2} \sigma}{d \Omega_{\pi}^{*}}=J\left(t, \phi_{q \pi}^{*} \rightarrow \cos \theta_{q \pi}, \phi_{q \pi}\right) \frac{d^{2} \sigma}{d t d \phi} \cdot \frac{d t}{d \cos \theta^{*}}
$$

where $\frac{d t}{d \cos \theta^{*}}=2 p^{*} q^{*}$ is the Jacobian factor. The form of the Jacobian factor results from the equivalence for a boost along $\mathbf{q}$ to its center of mass quantity. The full derivation of the Jacobian for nonstationary targets (i.e. $A>1$, when transverse components of the pion momentum have to be included in the transformation) can be found in Appendix B of reference [55]. This derivation in general makes use of 
the fact that energy and momenta are independent of the pion angle in the center of mass frame. Note that the transformation between spherical and spectrometer coordinate systems is taken into account by a second Jacobian.

\subsection{Spectrometer Models}

After the angles and momenta for each event are generated at the vertex, the events are sent to the single arm subroutines which transport the particles through the magnetic fields in the spectrometer using a COSY INFINITY model [95]. The COSY model consists of matrix elements which transport the particle sequentially through the magnetic optics in the spectrometer. The sequential transport entails transporting the particle from aperture to aperture such as from the target to the entrance of the first magnet, from there to the center of the magnet, from there to its exit and similarily for all subsequent spectrometer apertures. The sequential implementation of the COSY model is advantageous in terms of allowing for the modeling of hadron decay. The model of the magnets is generated using a combination of field map data, and comparisons with actual data.

Comparing simulated reconstructed quantities to experimental data can be used as a check on cross section and spectrometer optics models. Since a cross section weight is applied to each event, the agreement of the distributions of the one dimensional projection of physics quantities like $Q^{2}$ or $W$ yields information about the description of the kinematic dependence of the cross section model used. In addition, a comparison of the reconstructed spectrometer quantities provides a good check of the reconstruction matrix elements (see Figure 4.1). While the 

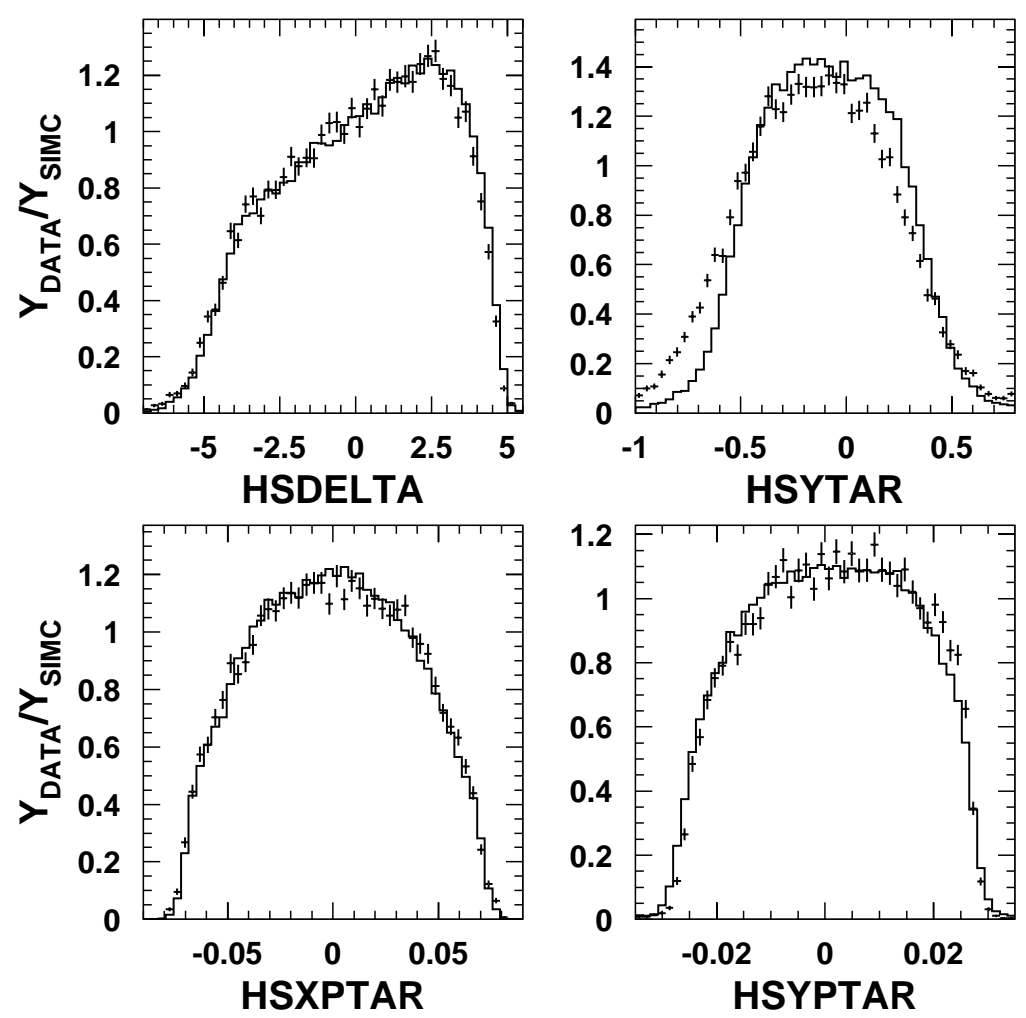

Figure 4.1: Comparison of data (crosses) and SIMC (solid line) charge normalized counts for HMS reconstructed quantities. The fractional momentum hsdelta and the spectrometer angles hsxptar and hsyptar are used in the calculation of the relevant physics variables. Generally, the Monte Carlo describes the data quite well except for small regions at the edges of the hsyptar acceptance. A similar effect was observed in elastic electron data.

SIMC reconstruction matrix is determined in a simultaneous multiple parameter fitting procedure [96], the data reconstruction matrix is fitted sequentially with experimental optics data as described in Chapter 3. Since the optics matrix cannot rely on known optics quantities as in the forward/backward projection used in the COSY model, it is relatively sensitive to field offsets and effects that result in events populating regions for which no data exist to constrain the series expansion. Event recontruction from a region in the focal plane for which no description from the 
optics matrix exists may appear as significant discrepancies in $X^{\prime}, Y_{t a r}$ and/or $Y_{t a r}^{\prime}$ between experimental data and Monte Carlo. Such an acceptance mismatch was found in the HMS $Y_{t a r}^{\prime}$ acceptance as discussed in Chapter 3.

An offset in the magnetic elements of the spectrometer appears as a correlation between focal plane and reconstructed quantities. As discussed in Chapter 3, such correlations were found between HMS and missing mass and $y_{f p}^{\prime}$ and missing mass, which were attributed to an offset in the magnetic field of the third quadrupole, Q3. This hypothesis was tested by simulating the effect of a Q3 offset in the Monte Carlo. To this end, the particle momentum was modified at the entrance of Q3 and adjusted back to its original value at the exit of Q3. Comparing the resulting simulated correlation in HMS $x_{f p}^{\prime} / y_{f p}^{\prime}$ and missing mass shows relatively good agreement with the observed effect in the data for a particle momentum modified by $0.3 \%$ at $\mathrm{p}_{H M S}=4.5(\mathrm{GeV} / \mathrm{c})$. In order to treat Monte Carlo and experimental data equivalently, the offset is included in the forward going Monte Carlo, and both types of data are corrected by the HMS $\left(x_{f p}^{\prime}, \delta\right)$ and $\left(y_{f p}^{\prime}, \delta\right)$ corrections described in Chapter 3 .

\subsection{Passage Through Materials}

To model particle events realistically, SIMC includes the effect of energy losses and multiple scattering on the kinematics as particles traverse various materials. 


\subsubsection{Ionization Energy Loss}

Ionization energy loss of the incoming and outgoing electron and hadron occurs primarily in the target due to ionization of atoms in the material traversed. The average energy loss by charged particles in matter is usually expressed in terms of the Bethe-Bloch formula. However, it can be shown that the energy loss probability distribution is asymmetric at decreasing absorber thickness and at high energies. To take into account fluctuations around the mean energy loss the distribution is in fact better described with a Landau distribution as explained in detail in reference [97]. The energy loss function is determined by two parameters, the most probable energy loss $\left(E_{\text {prob }}\right)$, and the full width at half maximum of the distribution $(\xi)$. In SIMC the energy loss is calculated using a Landau distribution function.

The most probable energy loss can be calculated from a random number, $\lambda$, obtained from a Landau distribution, and can be written,

$$
E_{\text {prob }}=\lambda \xi+E_{\text {true }}
$$

where $\xi=\frac{2 \pi N_{A} z^{2} e^{4}}{m_{e} c^{2}} \frac{Z t}{A \beta^{2}}$ and where $N_{A}$ is Avogadro's number, $z e$ is the charge of the incident particle, $m_{e} c^{2}$ denotes the electron mass, $t$ is the material thickness in $\mathrm{g} / \mathrm{cm}^{2}, Z$ and $A$ are the atomic number and mass of the material and $\beta$ denotes the velocity of the incident particle in units of $\mathrm{c}$. The Landau distributed random number is obtained from the standard Landau distribution and can be expressed in terms of a Gaussian distributed number $x$ as outlined in references [55, 98].

In SIMC the incident electron is followed through the target cell wall as well 
as the cryogen and target exit window and the relevant energy loss is calculated. The energy loss of the outgoing electron and hadron upon traversing various materials from the target to the spectrometer windows and the passage through the spectrometer exit window is also determined. The general procedure is consistent with the one used in the data analysis (see reference [99]) in terms of correcting the incident and scattered electron energies as well as the hadron energy for energy loss in the reconstruction of events. However, while SIMC calculates the most probable energy loss, the data analysis applies the average value of the energy loss. It can be shown, however, that the difference in the correction is relatively small $(\approx 1-2$ $\mathrm{MeV}$ ) and does not affect the comparison of simulated and experimental data.

\subsubsection{Multiple Scattering}

The measured experimental resolution as determined by the wire chambers is modeled in SIMC and includes the effect of Coulomb multiple scattering in the target and spectrometers. The deflection of the original scattering angle of a particle traversing a medium can be calculated from a random Gaussian distributed number and the width of a Gaussian distribution describing multiple scattering,

$$
\theta_{0}=g \times \frac{13.6 \sqrt{t}[1+0.038 \ln (t)]}{\beta p}
$$

where $\beta$ and $p$ denote the velocity and momentum of the incident particle in units of $\mathrm{MeV}$ and $t$ is the thickness of the scattering medium in radiation lengths. Note that $g$ is a uniformly distributed real number between 0.0 and 1.0. The materials 
traversed by the particles in either spectrometer are included in the calculation and are listed in Table 3.6.

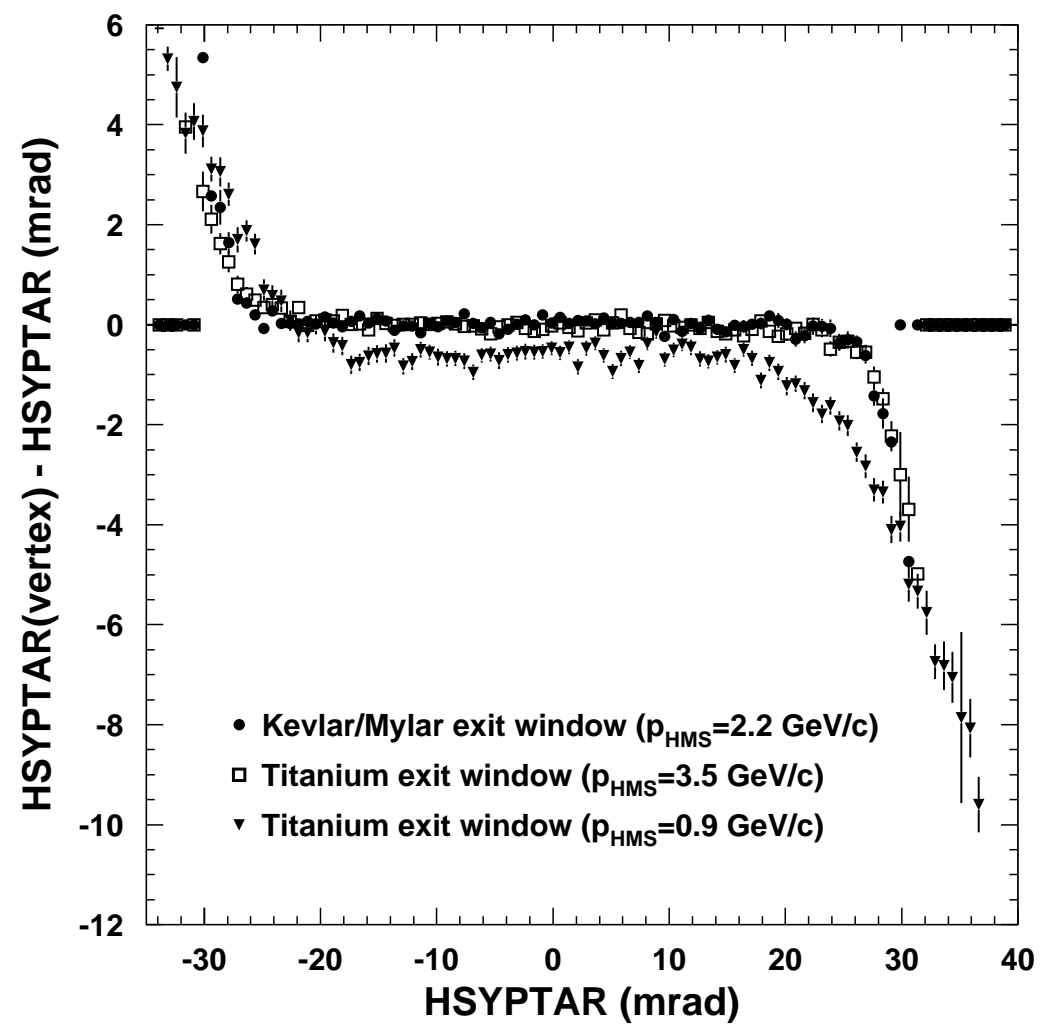

Figure 4.2: Simulated HMS $Y_{\text {tar }}^{\prime}$ acceptance for a point target for different spectrometer exit window thicknesses. The HMS matrix elements were fit using a window made of Kevlar/Mylar $\left(X_{0}=53.3\right)$, while the exit window used in this experiment is made of Titanium $\left(X_{0}=3.56\right)$. Multiple scattering is present in all three cases, but has increased in the case of the thicker Titanium window at low HMS momenta. The size of the effect also depends on the target length along the beam and HMS central angle.

The effect of multiple scattering is calculated in SIMC for both the incident and scattered electrons and also for the produced hadron. After the inclusion of multiple scattering, the experimental and simulated resolutions agree to $\approx 30 \%$. Although this deviation appears to be rather large, it is within the acceptable experimental 
limits. The effect of changing the simulated resolution to match the experimental one precisely has been tested with elastic singles electron data and was found to have a relatively small effect on the simulated acceptance.

Multiple scattering has increased in the HMS relative to earlier experiments due to the larger thickness of the Titanium spectrometer exit window. The effect can be observed in SIMC, since one has access to both the vertex and the reconstructed quantities. Figure 4.2) shows the result of study of the $Y_{t a r}^{\prime}$ acceptance for different HMS central momenta and spectrometer exit window thicknesses. Multiple scattering is present in all three cases, but it has significantly increased at $P_{H M S}=0.9$ $(\mathrm{GeV} / \mathrm{c})$. At higher momenta $\left(P_{H M S}=3.5(\mathrm{GeV} / \mathrm{c})\right.$ for $\left.\mathrm{F}_{\pi}-2\right)$, the reconstruction is not influenced noticably. Note that the HMS reconstruction matrix was fitted at HMS central momentum $P_{H M S}=2.2(\mathrm{GeV} / \mathrm{c})$.

\subsection{Pion Decay}

The possibility of pion decay in flight is included in the Monte Carlo. Charged pions predominantly decay via $\pi^{ \pm} \rightarrow \mu^{ \pm} \nu_{\mu}$ with a branching fraction of $99.99 \%$. The fraction of pions decaying in flight on their way from the target can be calculated from the spectrometer central momentum and path length and was found to be $\approx$ $15 \%(13 \%)$ at $P_{\pi}=2.931(3.336) \mathrm{GeV} / \mathrm{c}$. Note that these pions will not produce a trigger since they are lost before reaching the third layer of scintillators.

Two different processes are of concern in the pion decay correction to the data. The most straightforward correction entails the correction for the total number of events lost due to in flight decay of the pion with none of the decay products within 
the spectrometer acceptance. However, there is a nonzero probability that a muon resulting from pion decay will produce a valid trigger. Implementing a correction for this case is complicated by the fact that muon tracks do not necessarily reconstruct to the true pion interaction vertex at the target, thus resulting in a misrepresentation of the reconstructed target variables. In fact, muons produced in pion decay constitute a background that cannot be eliminated by particle identification. Comparing the pion $\left(m_{\pi}=0.139 \mathrm{GeV}\right)$ and muon mass $\left(m_{\mu}=0.105 \mathrm{GeV}\right)$ suggests that there is no possibility to distinguish particle types by their time of flight. It is therefore not feasible to treat all pion decay events with a common correction factor neglecting the presence of muon tracks.

SIMC simulates both the number of decayed pions and the resulting muon trajectory. The location of the pion decay is determined from the probability of pion decay within a distance, $Z_{\text {decay }}$, which is calculated in regular intervals along the particle trajectory to the detectors. These intervals are defined by the apertures for which the COSY transport matrix elements are evaluated. In particular, the probability of a pion decaying in such an interval can be expressed in terms of

$$
P_{\text {decay }}=1-e^{-\frac{Z}{\beta \gamma c \tau}}
$$

where $Z$ denotes the distance between two apertures, $c \tau=780.45 \mathrm{~cm}$ is the pion decay length in its rest frame and the factor $\beta \gamma$ is the relativistic transformation to the laboratory frame. For each tranformation between apertures the location of the pion decay is then determined from a comparison of the calculated decay distance and the 
pathlength between elements. For example, if the pion decay distance is within the distance between apertures the pion is forced to decay and the particle trajectory of the decay product is used for subsequent calculations. If the decay occurs in a magnetic field, the decay vertex is assumed at the location of the next available matrix element calculation. The order of particle decay and transport is determined by the midpoint between apertures. While the resulting muon is transported for the case of pions decaying prior to reaching the midpoint, pions with larger decay distances are transported first and then forced to decay.

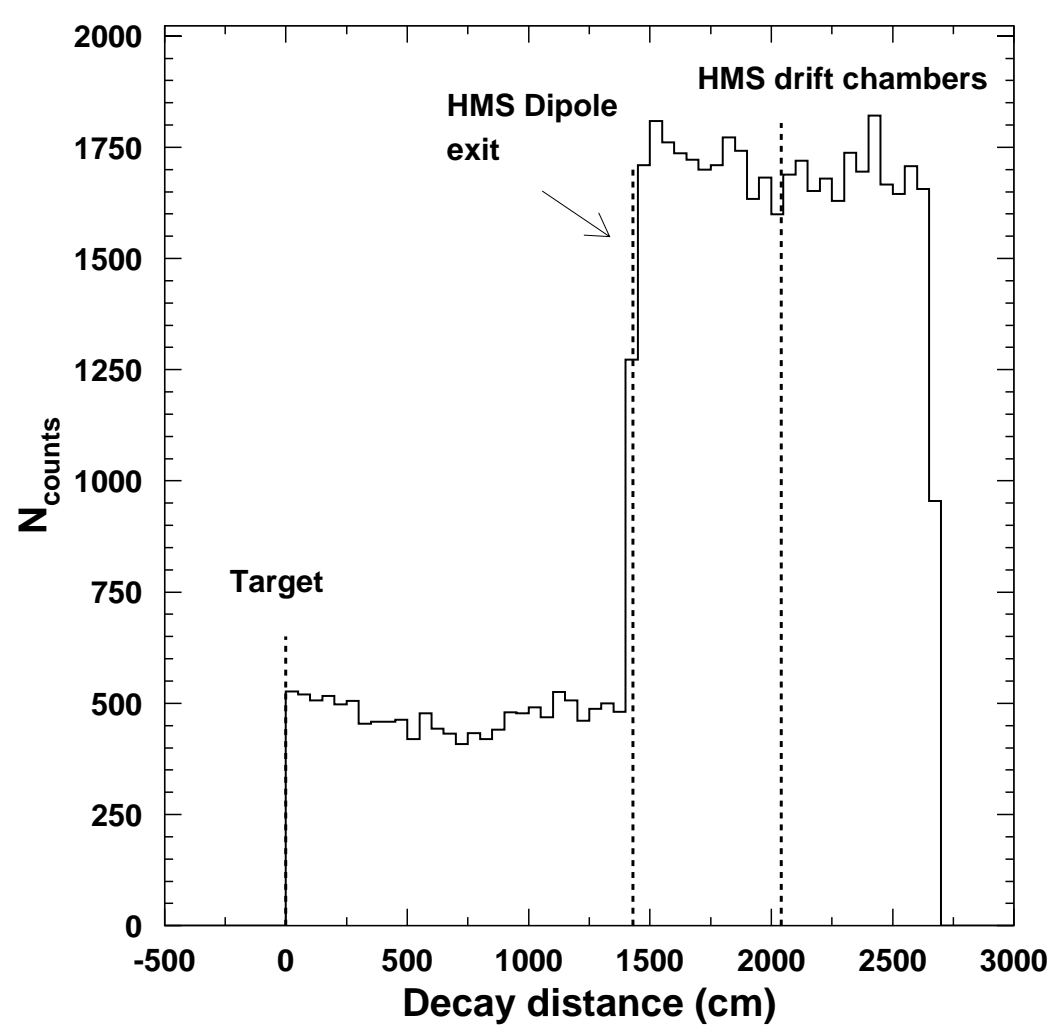

Figure 4.3: Simulated HMS pion decay distance for pions that decay and for which the resulting muon falls within the acceptance and passes all cuts. A large fraction of all simulated muons that pass all cuts come from the region after the HMS dipole and inside the spectrometer hut. 
While a large fraction of all pions decay in flight, the number of events for which the resulting muon falls in the spectrometer acceptance is relatively small. A large fraction of pion decay events that generate a valid trigger result from pion decay close to the target or pion decay in the field free region after the HMS magnetic elements and inside the spectrometer hut (see Figure 4.3). The reduced acceptance for events from the quadrupoles may be due to the type of muon trajectory that results from a pion decaying within these magnets. Given the design of the HMS, it seems reasonable that the probability for an event with a trajectory not originating from the target to be accepted is relatively low. On the other hand, if a particle decays within the dipole, the spectrometer optics may be conducive to guide the decay product into the detector hut. Events that decay after $\approx 20 \mathrm{~m}$ are in the detector hut beyond the drift chambers and are more likely to generate a valid trigger. The pion decay fraction of the total accepted number of events is $\approx 3.96$ $\%$. Note that this value includes pions decaying after the drift chambers.

As mentioned above the separation of pion and muon events is not feasible experimentally and the absolute number of pions lost must be estimated. However, the total number of events lost in pion decay is directly related to the HMS central momentum and the fractional deviation from the central ray. Since the pion fractional momentum is similar between high and low epsilon settings, the pion decay fraction can be assumed to be constant for a particular value of $Q^{2}$. The random uncertainty between $\epsilon$ settings is taken to be $0.03 \%$ to account for muons coming from pions normally outside the acceptance. The overall uncertainty due to the simulation of pion decay is taken to be $1 \%$. 


\subsection{Radiative Corrections}

Radiative corrections describing the emission of real or virtual photons by the charged particles involved in the reaction are an important part in the analysis of electron scattering experimental data. Traditionally, the radiative correcting of experimental data entailed calculation of a correction factor in terms of missing energy or missing mass to account for the re-distributed strength in cross section. However, the validity of this correction factor is limited to the central kinematic values and does not account for variation of the true unradiated cross section accross the experimental acceptance. A way to address this shortcoming is the direct calculation of cross section spectra using a Monte Carlo which includes radiative processes as described in detail in reference [100].

The radiative corrections used in this analysis are based on a formalism derived by Mo and Tsai [101]. The original formalism was derived for radiative corrections applied to inclusive elastic electron scattering of electrons from protons and was extended for coincidence $\left(e, e^{\prime} p\right)$ reactions $[102,100]$. To accomodate pion electroproduction, the implementation of radiative processes in SIMC was further modified. The target particle is considered to be a stationary proton and the final pion is taken to be an offshell proton. An alternative approach is to treat the target proton as an offshell pion.

Radiative effects in electron scattering result from the acceleration of charged particles in the presence of electric fields. In the case of external radiation one of the charged particles involved in the reaction emits a real photon upon interacting 
with the electric field of nuclei encountered while traversing a material. External corrections are relatively straightforward since the particles radiate independently eliminating interference terms in the summation of the amplitude. In contrast, the case of internal radiation in which charged particles radiate in the field of the primary target nucleon is complicated by various interference terms resulting from coherent addition of the amplitudes. Internal corrections also contain second order diagrams such as vacuum polarization and self energy diagrams. However, only infrared (IR) divergences that cancel the IR divergent terms in the first order internal corrections are kept. The first order bremsstrahlung interference terms and second order diagrams are shown in Figure 4.4 and Figure 4.5.

The radiative corrections implemented in SIMC include approximations to the photon energy and the angular distribution of radiated photons. The restriction of values of radiated photon energy to much less than the energies of the inital and final state particles is referred to as the soft photon approximation. In this limit the fundamental one photon exchange amplitude factorizes from other radiative processes. The extended peaking approximation provides an important simplification for the calculation of radiative effects in the coincidence framework. In this approximation the single photon bremsstrahlung radiation is divided into three discrete photon directions (along the direction of incoming electron and scattered electron and pion momentum). The total radiated strength in this limit is preserved by dividing the nonpeaked terms of the angular distribution evenly between the electron peaks.

To account for emission of bremsstrahlung photons with respect to the directions of incoming electron and scattered electron and pion, the first order pion 
electroproduction cross section is modified by a multiplicative radiative weight factor, $R_{\text {corr }}$,

$$
R_{\text {corr }}=\left(1-\delta_{\text {hard }}\right) \delta_{\text {soft }} \Phi_{e}^{e x t} \Phi_{e^{\prime}}^{e x t}
$$

The internal hard contribution can be expressed in terms of the second order virtual photon diagrams in the limit $Q^{2}>>m^{2}$,

$$
\delta_{\text {hard }}=\frac{2 \alpha}{\pi}\left(\left(1-\frac{3}{4} \ln \left(\frac{Q^{2}}{m^{2}}\right)_{\text {vertex }}\right)+\left(\frac{5}{9}-\frac{1}{3} \ln \left(\frac{Q^{2}}{m}\right)\right)_{v p}\right)
$$

where IR divergences in the two-photon exchange and self-energy diagrams are eliminated with the corresponding terms in the first order internal correction. The soft correction factor is given in terms of internal bremsstrahlung and external contributions along the direction of incoming and scattered electron,

$$
\begin{aligned}
\delta_{\text {soft }} & =\frac{1}{\Gamma\left(1+b t_{e}\right)} \frac{b t_{e}+\lambda_{e}}{k_{e}^{b t_{e}}}\left(\sqrt{k_{e} k_{e}^{\prime}}\right)^{\lambda_{e}} \frac{1}{E_{e}^{1-\lambda_{e}-b t_{e}}} \\
& \times \frac{1}{\Gamma\left(1+b t_{e}^{\prime}\right)} \frac{b t_{e}^{\prime}+\lambda_{e}^{\prime}}{k_{e}^{b t_{e}^{\prime}}\left(\sqrt{k_{e} k_{e^{\prime}}}\right)^{\lambda_{e}^{\prime}}} \frac{1}{E_{e^{\prime}}^{1-\lambda_{e}^{\prime}-b t_{e}^{\prime}}} \\
& \times \frac{\lambda_{\pi}}{\left(\sqrt{M_{p} k_{\pi}}\right)^{\lambda_{\pi}}} \frac{1}{E_{\pi}^{1-\lambda_{\pi}}},
\end{aligned}
$$

where the factors $b t_{i}$ denote the screening properties of material $i$ in the calculation of external bremsstrahlung. The parameter $b$ depends on the atomic charge, $Z$, of the material and $t$ is the thickness of the material traversed in radiation lengths. The contribution from pion radiation to external corrections is negligible relative to electron radiation since it is suppressed by a factor $\left(\mathrm{m} / \mathrm{m}_{\pi}\right)^{2}<10^{-4}$. The internal 
corrections are expressed in terms of the factor, $\lambda$,

$$
\begin{array}{r}
\lambda_{e}=\frac{\alpha}{\pi}\left(\ln \left(\frac{4 k_{e}^{2}}{m_{e}^{2}}\right)-1+2 \ln \left(\frac{k_{e}}{k_{e}^{\prime}}\right)+\ln \left(\frac{1-\cos \left(\theta_{e}\right)}{2}\right)\right) \\
\lambda_{e^{\prime}}=\frac{\alpha}{\pi}\left(\ln \left(\frac{4 k_{e^{\prime}}^{2}}{m^{2}}\right)-1+2 \ln \left(\frac{k_{e}}{k_{e^{\prime}}}\right)+\ln \left(\frac{1-\cos \left(\theta_{e}\right)}{2}\right)\right) \\
\lambda_{\pi}=\frac{\alpha}{\pi}\left(\ln \left(\frac{E_{\pi}}{E}+\left|\mathbf{p}_{\pi}\right| E_{\pi}-\left|\mathbf{p}_{\pi}\right|\right)-2\right)
\end{array}
$$

where the term $2 \ln \left(\frac{k_{e}}{k_{e^{\prime}}}\right)+\ln \left(\frac{1-\cos \left(\theta_{e}\right)}{2}\right)$ denotes the splitting of the nonpeaked contributions to the total strength between the incoming and scattered electron peaks in the peaking approximation. The multiplicative factors taking into account external radiation at large photon energies can be written,

$$
\Phi_{i}^{e x t}=1-\frac{b t_{i}}{b t_{i}+\lambda_{i}} \frac{E_{i}}{\left|k_{i}\right|}
$$

where $i$ denotes $e$ and $e^{\prime}$ respectively.

Radiative corrections are implemented in SIMC using randomly generated radiated photon energies in each direction and subsequent adjustment of the kinematics of the participating particles. The radiated photon energy along the directions of incoming electron, outgoing electron and outgoing pion is generated from a function of the form

$$
G=\frac{g E^{g-1}}{E_{\max }^{g}-E_{\min }^{g}},
$$

where $E_{\max }^{g}$ and $E_{\min }^{g}$ are the limits on the generated photon energy and the parameter $g$ is given by $\lambda+b t$. Note that the upper limit on the photon energy should 
be at least comparable to the maximum energy a particle can lose and be retained in the acceptance. Since there is a nonzero probability for photon radiation the lower limit can be taken to be zero. Once the radiated photon energy is known the kinematic properties of the particle of interest are adjusted on an event by event basis. To ensure that the new kinematics are physically allowed when the incident electron radiates, the kinematic properties of the event are re-calculated.

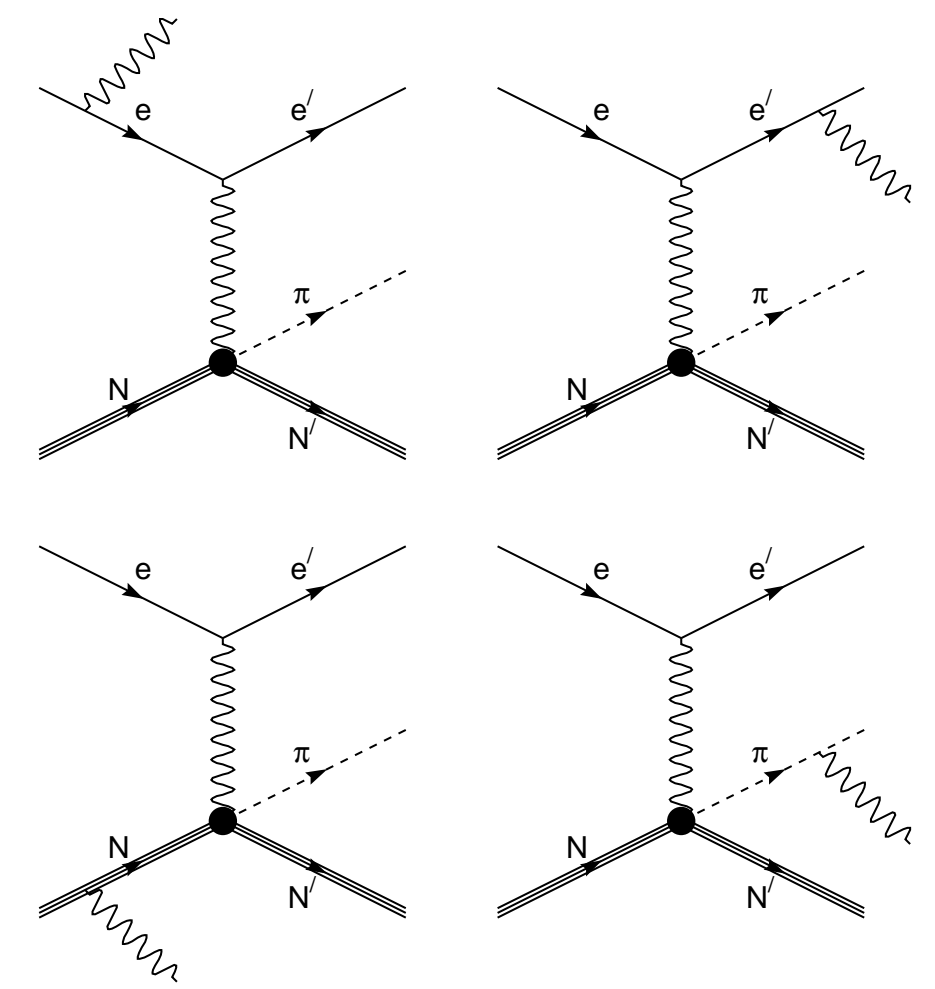

Figure 4.4: Feynman diagrams contributing to first order, internal, soft Bremsstrahlung radiation. Figure from reference [55].

The probability for a particle to radiate at a given energy between $E_{\min }$ and $E_{\max }$ is quantified in terms the radiation weight, $R_{\text {corr }}$, calculated for each event. While the hard correction and external radiation factors are calculated directly, the soft radiative weight is given by integration of the soft correction factor over the 
relevant energy range. The total soft radiative weight is then the product of the soft contribution from each direction. and the total radiative weight can be written

$$
W_{\text {rad }}^{\text {event }}=\left(1-\delta_{\text {hard }}\right) W_{\text {soft }}^{e} W_{\text {soft }}^{e^{\prime}} W_{\text {soft }}^{\pi} \Phi_{e}^{e x t} \Phi_{e^{\prime}}^{e x t} .
$$

The method described above has been tested in a comparison with $\left(e, e^{\prime} p\right)$ elastic data and found to agree relatively well [100]. Therefore, the global uncertainty in the method is taken to be $2 \%$ with an additional uncertainty of $1 \%$ to take into account the uncertainty associated with the extension of the formalism to pion electroproduction. The extra uncertainty is assigned based on the relative difference in results when treating the pion as an off-shell proton and the proton as a virtual pion. The radiative corrections are applied in the same way to both high and low epsilon settings and no significant dependence between points can be observed.
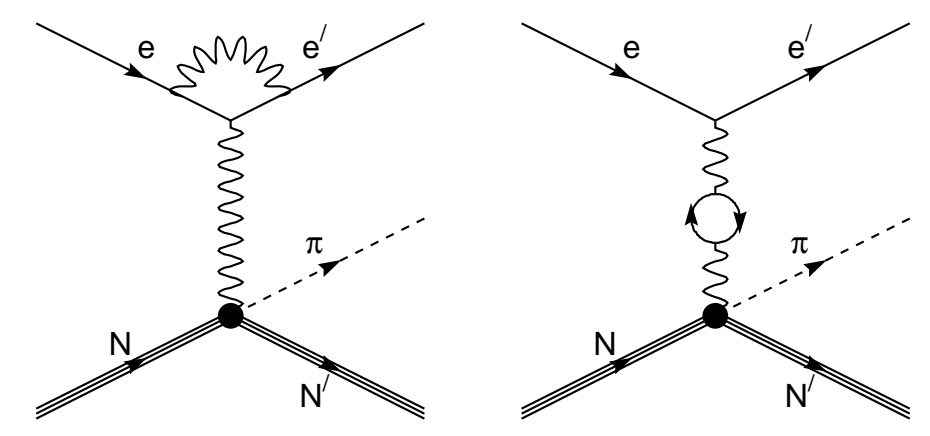

Figure 4.5: Feynman space diagrams representing virtual photon corrections to one photon exchange cross section. Figure from reference [55].

The random uncertainty due to the method is assigned from a study of the radiative tail in the $\mathrm{H}\left(e, e^{\prime} \pi^{+}\right)$reaction. The integrated ratio of data and SIMC was calculated as a function of missing mass cut and normalized to the largest missing 


\begin{tabular}{|l|c|c|c|}
\hline$Q^{2}(\mathrm{GeV} / \mathrm{c})^{2}$ & $\mathrm{M}_{x}(\mathrm{GeV})$ cut & $\epsilon_{\text {high }}$ (Data/SIMC) & $\epsilon_{\text {low }}$ (Data/SIMC) \\
\hline 1.60 & $0.92-0.98$ & 0.9840 & 0.9865 \\
& $0.92-1.00$ & 0.9918 & 0.9935 \\
& $0.92-1.02$ & 1.0 & 1.0 \\
2.45 & $0.92-0.98$ & 0.9835 & 0.9874 \\
& $0.92-1.00$ & 0.9915 & 0.9917 \\
& $0.92-1.02$ & 1.0 & 1.0 \\
\hline
\end{tabular}

Table 4.1: Comparison of data to Monte Carlo as a function of missing mass $\left(M_{x}\right)$ cut in the radiative tail. The ratios are normalized to 1.0 at the largest missing mass cut. Note that the region above 1.02 GeV up to two pion threshold is excluded to avoid contamination of the sample from collimator punchthrough events.

mass cut. The region above 1.02 up to two pion threshold was excluded in this study to avoid contamination of the sample by pions passing through the collimator. The "collimator punch-through" events were simulated in SIMC and found to produce an anomalous peak centered in the region $M_{x}=1.025-1.07$. Although the overall cut dependence appears rather large, the dependence of the ratio on epsilon is relatively small (see Table 4.1). Based on the radiative tail studies a random uncertainty of $0.5 \%$ between epsilon settings is assigned conservatively.

The contribution from two-photon exchange diagrams is currently not included in SIMC. Although the effect of two photon exchange is usually discussed in the context of extracting the proton charge form factor, it may also be relevant in the interpretation of other precision electron scattering data. In the case of the proton form factor a significant difference was found between measurements of $G_{E}^{p}$ from Rosenbluth separations and measurements from the ratio of transverse to longitudinal proton asymmetries. The difference appears most clearly above values of $Q^{2}=1$ $\mathrm{GeV}$ in agreement with decreasing size of the electric contribution to the cross section ratio. In contrast, the contribution to the transverse/longitudinal asymmetry 


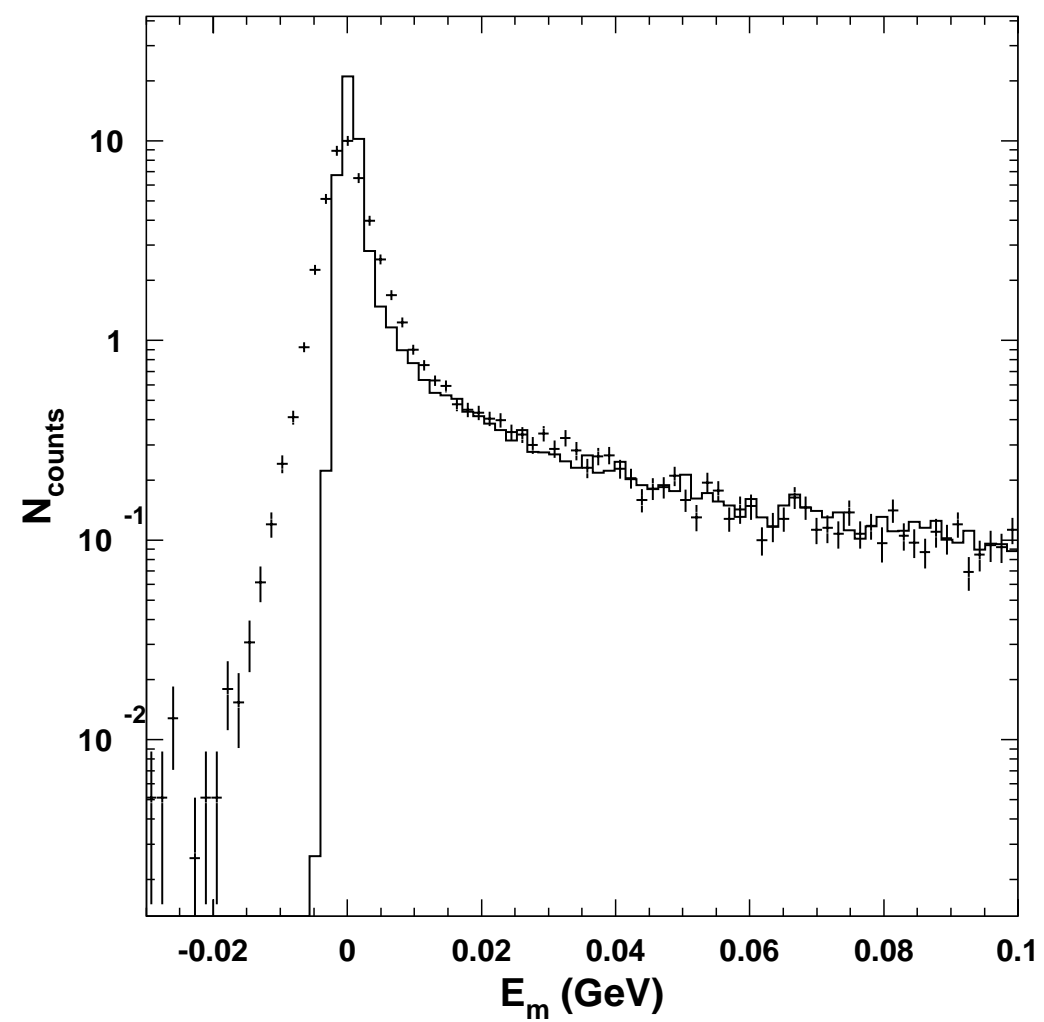

Figure 4.6: Missing energy distribution for one of the ${ }^{1} H\left(e, e^{\prime} p\right)$ kinematics. Elastic data are used to distinguish resolution from radiative effects. In the elastic case the Monte Carlo simulation (solid line) describes the data (crosses) in the radiative tail quite well. The data/SIMC ratio changes by $1 \%$ over the entire missing energy acceptance. The discrepancy at low missing energy is due to resolution effects.

is still lowest order giving support to the two photon exchange hypothesis. The consistency of the data after re-analysis of electron scattering data seems to suggest a problem in the approximations typically used in electron scattering, e.g. single photon exchange and radiative corrections based on Mo and Tsai. Due to the lack of data allowing for the determination of the effect of the two photon exchange on this analysis, all cross sections will be quoted in the one photon exchange framework. The order of magnitude of the systematic uncertainty due to the two photon 


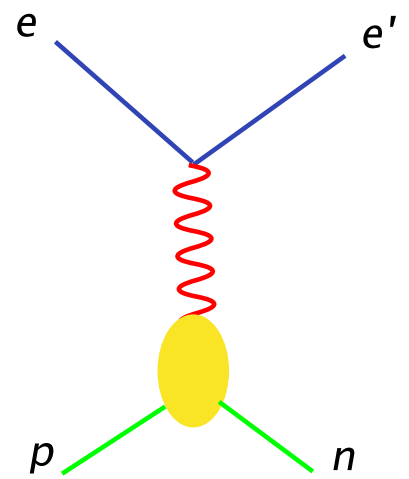

(a) One photon exchange.

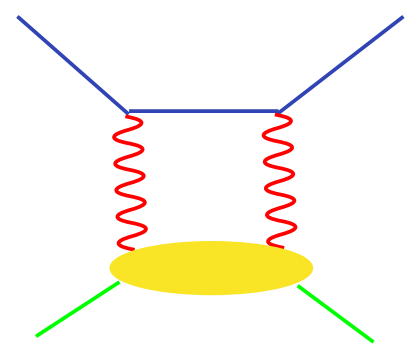

(b) Two photon exchange.

Figure 4.7: Feyman diagrams of the dominant contributions of the two photon exchange effect.

exchange can be estimated based on calculations for the proton electric form factor $[103,104]$. The effect on unseparated cross sections is on the order of $5 \%$ over the full epsilon range. However, the dynamical range in this analysis is smaller by a factor of two resulting in an epsilon separation of $\delta \epsilon \approx 0.3$ in the worst case. Also note that compared to the relative size of $G_{E}^{p}$ term in the cross section ratio $\sigma_{R}=$ $\left(\frac{\epsilon}{\tau}\right) \cdot\left(G_{E}^{p}\right)^{2}+\left(G_{M}^{p}\right)^{2}$, where $\tau=Q^{2} / 4 M^{2}$, pion electroproduction is dominated by the longitudinal piece of the cross section at small values of $t$. The elastic cross section results at $Q^{2} \approx 3.2$ for the epsilon range of interest $(\epsilon=0.3-0.6)$ for all available calculations gives a total size of the effect of $(1.1 \pm 0.2) \%$. Taking into account the mass difference of proton $\left(\mathrm{m}_{p}=0.938272 \mathrm{GeV}\right)$ and pion $\left(\mathrm{m}_{\pi}=0.1395699 \mathrm{GeV}\right)$ results in a factor of $\approx 6.75$ entering in the mass logarithm in the radiative correction calculations. This suggests that the size of the two photon effect estimated from elastic $e-p$ scattering should be further reduced by a factor of $\ln (6.75)=1.91 \approx 2$. Taking into account this additional reduction results in an estimated size of the two 
photon effect of $(0.5 \pm 0.1) \%$.

\subsection{Elastic Scattering}

Elastic scattering data provide a good check for the accuracy of the spectrometer acceptance and various effects on reconstruction such as radiative processes, multiple scattering and energy loss that are simulated in SIMC. The Monte Carlo can simulate both single arm and coincidence elastic scattering. In both cases all kinematic quantities are calculated from the simulated in- and out-of-plane angles of the scattered electron. Verification of the experimental yields is done by comparing the well known elastic cross section. The model cross section in SIMC is given by,

$$
\frac{d^{2} \sigma}{d \Omega_{e}}=\frac{\alpha^{2} \cos ^{2} \frac{\theta_{e}}{2}}{4 E^{2} \sin ^{4} \frac{\theta_{e}}{2}} \frac{E^{\prime}}{E}\left(\frac{G_{E_{p}}^{2}+\tau G_{M_{p}}^{2}}{1+\tau}+2 \tau G_{M_{p}}^{2} \tan ^{2} \frac{\theta_{e}}{2}\right)
$$

where $\theta_{e}, E$, and $E^{\prime}$ denote the electron scattering angle, incident electron energy and final electron energy. The electric and magnetic form factors are calculated from J.R. Arrington's parametrization to all available data [105].

$$
\begin{aligned}
& G_{E_{p}}^{2}\left(Q^{2}\right)=\frac{1}{1+3.226 Q^{2}+1.508 Q^{4}-0.3773 Q^{6}+0.611 Q^{8}-0.1853 Q^{10}+0.01596 Q^{12}} \\
& G_{M_{p}}^{2}\left(Q^{2}\right)=\frac{1}{1+3.19 Q^{2}+1.355 Q^{4}+0.151 Q^{6}-0.0114 Q^{8}-0.000533 Q^{10}-0.000009 Q^{12}}
\end{aligned}
$$

This parametrization extends the dynamic range of the alternative parametrization by P.E. Bosted [106] to $Q^{2}=30(\mathrm{GeV} / \mathrm{c})^{2}$ and is based on a global analysis of true $\mathrm{L} / \mathrm{T}$ separated cross section data. 
During $\mathrm{F}_{\pi}-2$, elastic data were taken at four different beam energies with a range in $Q^{2}$ between 2.41 and $5.42(\mathrm{GeV} / \mathrm{c})^{2}$. Electrons were detected in the SOS and the recoiling proton was detected in the HMS. In all four measurements the central spectrometer angle and momentum were matched so that the fractional momentum, $\delta$, for the elastically scattered electron and recoiling proton were centered at zero. A list of the kinematics can be found in Table 3.1 .

In order to compare experimental data to the Monte Carlo, the data were normalized by the total accumulated charge corrected for tracking and particle identification efficiencies and computer and electronic deadtimes. Standard cuts were applied to the reconstructed spectrometer variables $\left(\delta, X^{\prime}, Y^{\prime}\right)$. Further cuts were placed on the invariant mass, SOS Cerenkov and total energy deposition in the calorimeter to isolate true elastically scattered electrons. The contribution from the target cell walls was estimated from data taken with the aluminium dummy target at each setting. This contribution was subtracted using the method described in section 3.4.4.

Elastic coincidence data require an additional correction. Due to hadronic interactions in the spectrometer the number of recoil protons detected in the HMS will be reduced relative to the total number of elastic events. This is taken into account by a proton absorption correction applied to the data. To estimate the fraction of protons absorbed in the materials as they travel from the target to the scintillator planes used in the trigger, the probability of absorption was estimated from the mean free path between collisions and the thickness of a particular material traversed. The mean free path is calculated from the absorption cross section, since 
a large fraction of elastically scattered events is still expected to give a valid trigger. The values for the mean free path and density listed in Table 3.6 were taken from reference [97]. Similar to the pion transmission correction, the proton transmission can then be expressed in terms of the mean free path,

$$
T_{p}=e^{-\Sigma \frac{X_{i}}{\lambda_{i}}}
$$

where $X_{i}$ and $\lambda_{i}$ denote the thickness (in radiation lengths) and the interaction length of the $i^{\text {th }}$ material encountered in the particles path. The interaction length, $\lambda$ for each material is taken from the parametrization in reference [97].

The proton transmission can be determined experimentally from the ratio of coincidence events and the total number of electrons detected in the SOS,

$$
T_{p}^{e x p}=\frac{N_{\text {coin }}}{N_{\text {coin }}+N_{e^{-} \text {non-coin }}},
$$

where $N_{\text {coin }}$ and $N_{e^{-} \text {notcoin }}$ denote coincident and SOS non-coincident electrons respectively. The quantities $N_{\text {coin }}$ and $N_{e^{-} \text {notcoin }}$ are determined by applying cuts to the electron variables to constrain the proton kinematically within the HMS acceptance. This method is described in detail in reference [107]. Additional cuts were placed on acceptance, invariant mass, particle identification and SOS $Y_{\text {tar }}$ to reduce target cell wall contribution. The measured proton transmission was found to be $0.945 \pm 0.012 \%$ which is in relatively good agreement with the theoretical prediction of 0.935 . It should be noted the proton transmission is smaller by $\approx 1.5 \%$ 
relative to reference [108]. This is due to the addition of the aerogel detector and the replacement of the HMS spectrometer exit window with Titanium instead of the kevlar/mylar. The dominant contribution to the reduction in proton transmission comes from the aerogel detector $(1.3 \%)$.

Another important factor in the elastic analysis is the modification of the prescale factor from the programmed value whenever singles and coincidences are present. Generally all coincidences are prescaled in single arm elastic data and the prescale factor on the singles events is designed to accept every $i^{\text {th }}$ event (where $i$ is the value of the prescale factor). However, in the $\mathrm{F}_{\pi}-2$ data taking the prescale factor in three of the four settings was not large enough to eliminate all coincidences. Thus the programmed prescale factor generally overestimates the actual value for singles events. The effective prescale factor can, however, be calculated from the information in the scaler file, since all trigger and raw ADC information is written out for singles and coincidences.

The results for data and Monte Carlo yield ratios for all kinematic settings are shown in Figure 4.8. The data were analyzed using the scattered electron and coincidence events separately. The comparison of experimental to simulated yield ratios suggest a good understanding of the experimental acceptance and overall normalization.

The understanding of the HMS was tested using elastic single arm data. During $\mathrm{F}_{\pi^{-}}$2, elastic electron singles data were taken at the beginning of each of the four kinematic settings. The 4 pass data set consists of a series of runs for which the angle and momentum of the HMS were changed so that the central HMS delta for 

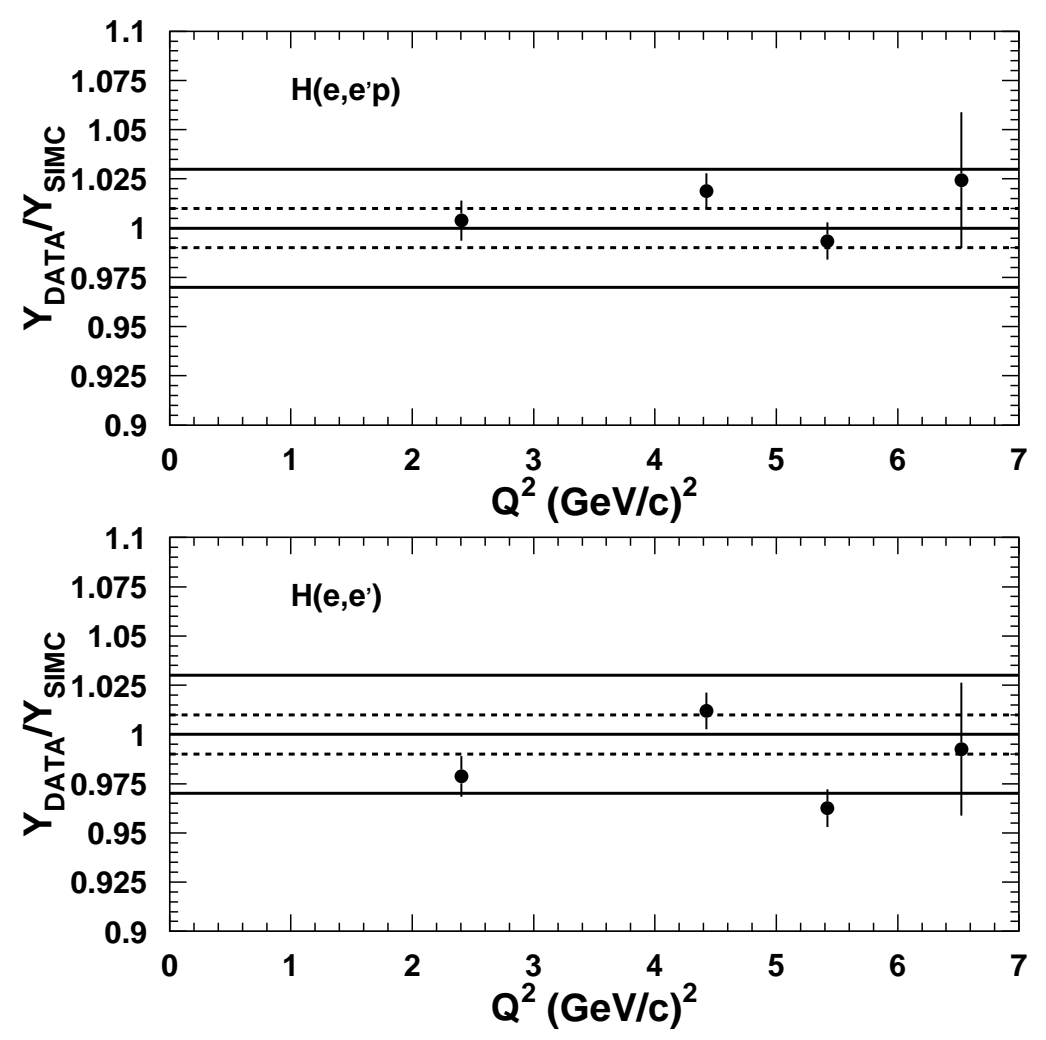

Figure 4.8: Ratio of data to Monte Carlo yields for elastic data taken for the four $F_{\pi^{-}}$ 2 kinematic settings. The top panel shows the coincidence yields (proton absorption correction applied) and the bottom panel shows the electron yields. The error bars shown are statistical only. The dashed lines indicate the point to point errors between the data points and the solid lines indicate the overall systematic uncertainty.

the elastically scattered electron was located at zero. For the remaining three beam energies the spectrometer settings were changed in order to scan the HMS delta acceptance from $-4 \%$ to $+4 \%$. For this purpose the HMS central momentum was kept at a constant value while the angle was changed between $12^{\circ}-16^{\circ}$ and $20^{\circ}-28^{\circ}$ for two HMS delta scans per beam energy. These elastic data are well described by SIMC and the analysis suggests that the acceptance is not only understood at the central fractional $\delta$, but over the entire acceptance range covered in the HMS $( \pm$ 
$5 \%$ ) in this analysis. 


\section{Chapter 5}

\section{Experimental Cross Sections Overview}

The experimental cross sections are determined by comparing the experimental yields to those given by the Hall C Monte Carlo, SIMC. The Monte Carlo includes a model for the electroproduction cross section and a simulation of a variety of effects such as spectrometer acceptance, radiative corrections, pion decay, multiple scattering and energy loss as described in the previous chapter. If the simulation describes the experimental data properly, the experimental cross section can be extracted by iterating the model input cross section until best agreement between data and Monte Carlo is achieved. If the model input cross section describes the dependence on all kinematic variables $\left(W, Q^{2},-t, \theta, \phi\right)$ correctly, the experimental cross section can be extracted using

$$
\sigma_{\text {exp }}=\frac{Y_{\text {Data }}}{Y_{S I M C}} \cdot \sigma_{\text {model }}
$$

where $Y_{\text {Data }}$ is the charge normalized and efficiency corrected experimental yield integrated over the kinematic acceptance. $Y_{S I M C}$ is the equivalent Monte Carlo yield calculated for the same luminosity and using the model input cross section $\sigma_{\text {model }}$. The model cross section provides both bin centering corrections to the experimental cross section and the proper cross section weight factor for the acceptance.

Determining the experimental cross section is complicated due to the range covered in the kinematic variables and the correlations between these variables in a multi dimensional phase space. In general, the experimental cross section can be 
extracted from the average model input cross section and averaging over the acceptance. This approach requires knowledge of both the mean values of the relevant kinematic variables and the associated ranges. However, in an L-T separation the range of the kinematic variables of interest is often quite different at forward and backward electron kinematics (see Figure 5.1). Averaging over the full acceptance for high and low $\epsilon$ results in averaging over two different regions at each $\epsilon$ point and the separation of longitudinal and transverse cross sections becomes difficult to interpret. In particular, the kinematic range over which the separated cross sections have been averaged is not clearly defined. One way to address this issue is to restrict the kinematic phase space so that the integration is performed over the same region. Placing additional cuts on the experimental phase space results in a significant loss of statistics, which is not necessarily desirable in a precision cross section measurement. However, in this analysis the loss of statistics is outweighed by the requirements of extracting the cross section. For this purpose, the data are binned in $t$ and integrated in a multi-parameter phase space. Not all parameters are independent and in addition cover a different range in each $t$ bin. In the extraction, the cross section has to be averaged over the phase space and in order to do a meaningful L-T separation the same values have to be used at both high and low $\epsilon$ points.

The cross section extraction in the $\mathrm{F}_{\pi}-2$ analysis is done using a "bin-centering" method. This means that the cross section is calculated at a particular point in the experimental acceptance using a model input cross section for the description of the specific model cross section shape. The sensitivity of the cross section to the 


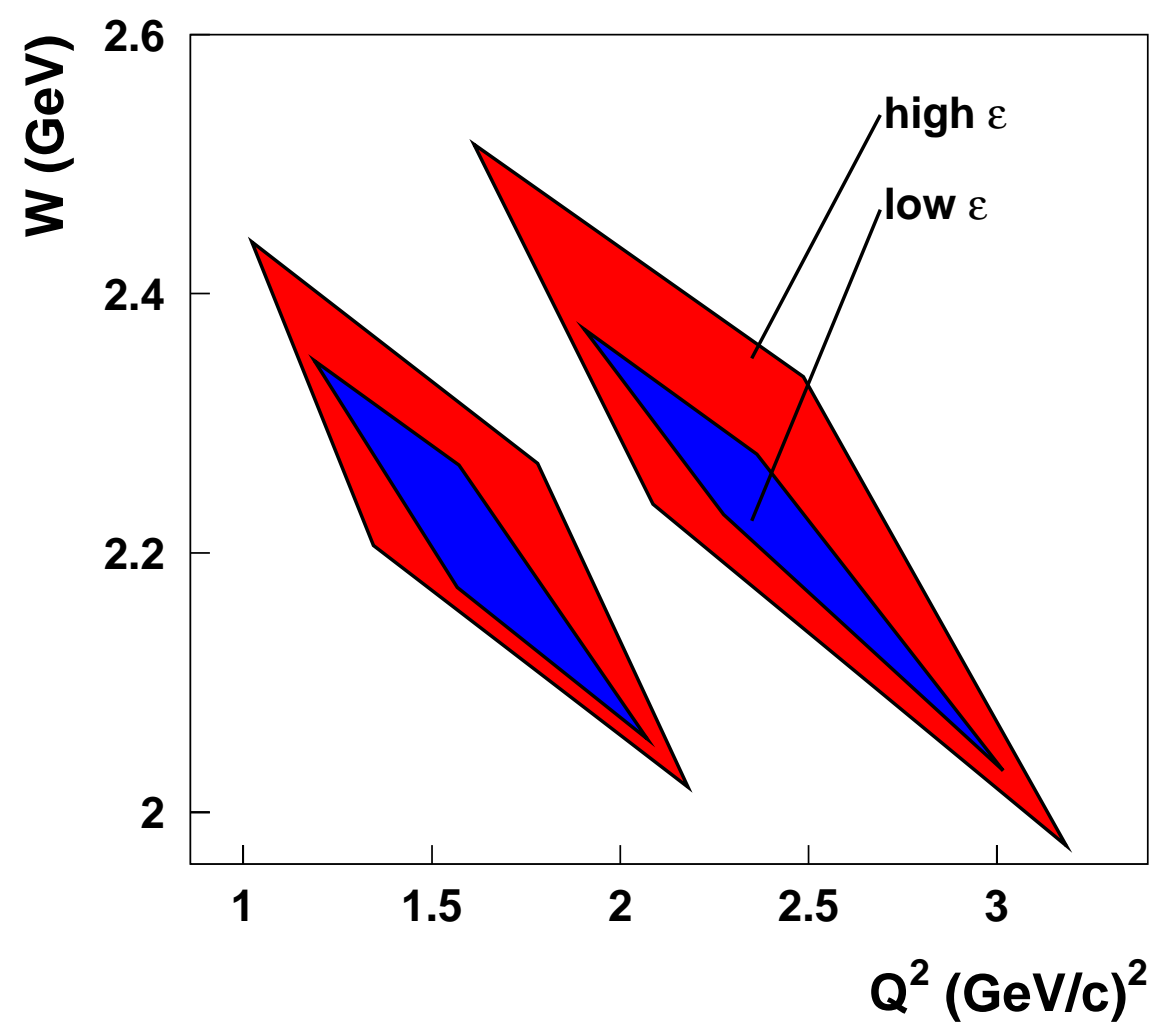

Figure 5.1: Phase space in $W$ and $Q^{2}$ at both low and high $\epsilon$ points for the two dynamic settings. Note that $-t$ and $\theta^{*}$ change in value as well across the $W-Q^{2}$ acceptance.

model input cross section across the entire acceptance is addressed in optimizing the kinematic dependences of the Monte Carlo to the data. The model cross sections are optimized in an iterative fitting procedure and the model dependence is given by the agreement with the final cross sections.

\subsection{Monte Carlo Equivalent Yield}

In order to extract the experimental cross section by comparing data to Monte Carlo, the equivalent SIMC yield has to be determined. The data yield is calculated in counts per $\mathrm{mC}$ requiring a normalization of the corresponding Monte Carlo yield 
to $1 \mathrm{mC}$. The Monte Carlo luminosity can be written,

$$
L=\frac{\rho t N_{A} N_{e}}{M}
$$

where $\rho$ is the target density in $\mathrm{g} / \mathrm{cm}^{3}, t$ is the target thickness in $\mathrm{cm}, N_{A}$ is Avogadro's number, $N_{e}$ is the total number of electrons normalized to $1 \mathrm{mC}$ of charge and $M$ is the target mass in amu. Given the luminosity factor the most general SIMC yield is given by,

$$
Y_{S I M C}=L \int_{V}\left(\frac{d^{6} \sigma}{d \Omega_{e} d E_{e} d \Omega_{\pi} d P_{\pi}}\right)^{\text {model }} A(V) R(V) J\left(\Omega \rightarrow d \mathbf{X}^{\prime}\right) d \mathbf{X}_{\mathbf{e}}^{\prime} d E_{e} d \mathbf{X}_{\pi}^{\prime} d P_{\pi}
$$

where $L$ is the experimental luminosity, $A$ is the coincidence acceptance function including energy loss, pion decay etc., $R$ is the radiative correction factor, $d \mathbf{X}^{\prime}=$ $d x^{\prime} d y^{\prime}$ is the differential solid angle in spectrometer coordinates and $J$ is the Jacobian transforming the model cross section from spherical to spectrometer coordinates which are used in event generation. The Jacobian can be expressed as

$$
J\left(\Omega \rightarrow \mathbf{X}^{\prime}\right)=\frac{1}{\left(1+\left(x_{e}^{\prime}\right)^{2}+\left(y_{e}^{\prime}\right)^{2}\right)^{3 / 2} \cdot\left(1+\left(x_{\pi}^{\prime}\right)^{2}+\left(y_{\pi}^{\prime}\right)^{2}\right)^{3 / 2}} .
$$

While radiative processes and acceptance effects are corrected for in the cross section extraction by explicitly including these effects in the Monte Carlo, other factors like final state interactions are not taken into account. This is relevant for pion electroproduction from targets other than hydrogen and will be discussed in subsequent 
sections.

The calculation of the SIMC yield for pion electroproduction from hydrogen targets is relatively easy because the pion's momentum is constrained by the electron kinematics and the scattered pion direction. The cross section becomes independent of pion momentum and can be expressed in terms of five independent variables. The resulting SIMC yield is,

$$
Y_{S I M C}=L \int_{V}\left(\frac{d^{5} \sigma}{d \Omega_{e} d E_{e} d \Omega_{\pi}}\right)^{\text {model }} A(V) R(V) J\left(\Omega \rightarrow d \mathbf{X}^{\prime}\right) d \mathbf{X}_{\mathbf{e}}^{\prime} d E_{e} d \mathbf{X}_{\pi}^{\prime}
$$

In contrast, in the case of pion electroproduction from deuterium an additional degree of freedom due to the relative momentum between nucleons has to be taken into account. To this end, the cross section can be expressed as the convolution of the $e-N$ cross section and the momentum space wave function of the struck nucleon. The resulting formalism is discussed in Chapter 5.3 in reference [55].

\subsection{Determining Cross Sections}

The extraction of the experimental cross section is complicated in this analysis due to correlations between kinematic variables and nonuniform angular acceptance. In order to evaluate the experimental cross section at a specific point at the center of the acceptance, the dependence of the cross section on all kinematic variables has to be well understood. In particular, to minimize the error due to averaging the measured yield in equation 5.1 across the acceptance in one or two kinematic variables, a phenomenological cross section model is used. This model can be shown to 
globally follow the $t$-, $Q^{2}$-, and $W$-dependence of previous experimental data. Note that the cross section model also includes a $\phi$ dependence. This is necessary because the experimental $\phi$ acceptance is not uniform. The contribution of the interference terms to the total measured cross section is not neglibible. The model was implemented in the Monte Carlo and the resulting simulated yields were compared to the experimental yields.

If the input model cross section describes the data well the experimental and simulated yields should be equivalent and their ratio should not show a significant dependence on a particular kinematic variable. However, even if the model correctly describes the kinematic dependence of the data, differences in overall scale cannot be excluded. To account for this difference in magnitude the model cross section is weighted by the measured experimental yield. The experimental cross section is then given by

$$
\left(\frac{d^{5} \sigma}{d \Omega_{e} d E_{e} d \Omega_{\pi}}\right)_{\bar{W}, \bar{Q}^{2}, \bar{\theta}, \bar{\phi}}^{e x p}=\frac{Y_{\text {Data }}}{Y_{S I M C}} \cdot\left(\frac{d^{5} \sigma}{d \Omega_{e} d E_{e} d \Omega_{\pi}}\right)_{\bar{W}, \overline{Q^{2}, \bar{\theta}, \bar{\phi}}}^{\text {model }}
$$

where $\left(\bar{W}, \overline{Q^{2}}, \bar{\theta}, \bar{\phi}\right)$ denotes the evaluation of the model cross section and in turn the extraction of the experimental cross section at a specific point in the acceptance. For the purpose of extracting the experimental cross section both high and low $\epsilon$ data are binned in 5 t-bins and $16 \phi$-bins and the cross section is evaluated at the center of each bin. The experimental and simulated yields are integrated over all other kinematic variables. The barred values in the expression above correspond to the acceptance weighted average values for each bin. For evaluating the "central" 
values a cut is placed on $W$ and $Q^{2}$ to define a common phase space at forward and backward electron kinematics. This particular choice of binning in a multidimensional phase space is not unique. For example, instead of binning the data in $t$ and $\phi$ one could bin the data in $W$ and $Q^{2}$.

In the analysis the experimental cross section is extracted using equation 5.6. The central cross section is evaluated using the same model as the one used in the Monte Carlo.

\subsection{Model Cross Section}

For the purpose of extracting the experimental cross section, the input model cross section has to be evaluated at a particular point in dynamic phase space. For pion electroproduction from hydrogen the virtual photon cross section is directly calculated given specific values for $Q^{2}, W$ and corresponding $\theta_{p q}$ or $-t$ and $\epsilon$ values at a given beam energy. The calculation of the model cross section in the case of deuterium is complicated due to the integration between the quasifree and the full six-dimensional picture.

Different methods are available for evaluating the central cross section. The first two methods calculate the central cross section directly in SIMC. In the first case the central values for the four momentum transfer to the nucleon are set in the Monte Carlo input and the corresponding central kinematic values are calculated. The central kinematics for the spectrometer detecting the electron are determined from the average values of $Q^{2}$ and $W$ for each such bin for which the cross section is to be calculated. In this approach the cross section is averaged over the out-of-plane 
angle. In the actual $\mathrm{F}_{\pi^{-}} 2$ data analysis these values are obtained from a standalone cross section calculation routine. This routine uses the same model as the one that is used in SIMC. By definition the results from the SIMC and standalone cross section central calculation agree exactly. In the case of deuterium the central cross section is averaged over the $-t$ acceptance at low and high epsilon points.

\subsection{Comparing Data and Monte Carlo}

The extraction of the experimental cross section requires a cross section model which describes the dynamic dependence of the data across the experimental acceptance. Given the constraint of previous pion electroproduction data, cross section model parametrizations can be developed for this purpose. However, the difference in experimental kinematics may result in insufficient description of the data in particular regions of phase space. To optimize the description of the data in the kinematic region of interest the cross section model is adjusted until agreement with the data is achieved. The alteration of the model cross section is done using an iteration procedure adjusting the individual response functions until no significant variation in the extracted cross section is observed. If the model input cross section already describes the data well, the difference between uniterated and iterated cross sections will be small. The result of the iteration procedure can then be used to estimate the model dependence of the extracted cross section. 


\subsubsection{Iteration of the Input Model Cross Section}

The starting pion electroproduction cross section model used in the simulation are previous experimental data. The individual response functions, $\sigma_{i}$ were optimized to follow the $t$ - and $Q^{2}$-dependence of the data. The dependence on $W$ was assumed to follow the phase space factor $\left(W^{2}-M_{p}^{2}\right)^{-2}$. Corrections to the $t$-dependence were applied to extend the validity of the model to higher $Q^{2}$ and to improve the agreement of the longitudinal term with results from VGL/Regge calculations in the large $Q^{2}$ limit.

The Monte Carlo input model cross section was iterated using a standalone FORTRAN program which incorporates a MINUIT routine for fitting corrections to the individual response functions in a $\chi^{2}$ minimization procedure [109]. In this method, the $t$ - and $Q^{2}$-dependence of each cross section term was fitted at each iteration. For example, the transverse piece of the model cross section can be written,

$$
\sigma_{T}^{\text {model }}=\left(\frac{A}{\bar{Q}^{2}}+\frac{B}{\bar{Q}^{4}}\right) \cdot f(\bar{W})
$$

where $A$ and $B$ are parameters indicating the relative strength of the $Q^{2}$-dependence and $f(W)$ is the phase space factor assumed for the $W$-dependence. The barred quantities denote $Q^{2}$ and $W$ at the center of the $t$ acceptance. The initial values for $A$ and $B$ are the ones that give the best description of previous $F_{\pi}$ data. The model

cross section, $\sigma_{T}^{\text {model }}$, result was then compared to the one extracted from experimental data and the parameters $A$ and $B$ were fit. For the best description of the data, the optimization was done simultaneously for all kinematic settings. The other 
three components of the pion electroproduction cross section were parameterized in a similar fashion (see section 5.4.2 for the functional form) and the corresponding parameters were fit. The resulting cross section terms can be denoted $\sigma_{i}^{i t}$, where $i \in$ (L,T,TT,LT). After all parameters are optimized, the iterated model cross section can be written,

$$
\left(\frac{d^{2} \sigma}{d t d \phi}\right)^{i t}=\left(\frac{d \sigma_{T}}{d t}\right)^{i t}+\epsilon\left(\frac{d \sigma_{L}}{d t}\right)^{i t}+\sqrt{2 \epsilon(1+\epsilon)}\left(\frac{d \sigma_{L T}}{d t}\right)^{i t} \cos \phi_{\pi}+\epsilon\left(\frac{d \sigma_{T T}}{d t}\right)^{i t} \cos 2 \phi_{\pi}
$$

where $\sigma_{i}$ are the iterated response functions with optimized parameters.

The experimental cross section was calculated at each iteration and the four response functions were fitted simultaneously for both $\epsilon$ points. This was done by fitting the experimental $\phi$ acceptance of the measured cross section for each $t$-bin to equation 5.8. The four response functions, L, T, TT and LT are the fit parameters in this case. The resulting four cross section terms were fitted to the model cross section in a multiple parameter fit as described above. The fit values for $\sigma_{i}$ were then used as an input in the next iteration calculation of the experimental cross section. The iteration has converged if the values of $\sigma_{i}$ (and the associated fit parameters) do not change any further in subsequent iterations. Typically, convergence is achieved within 1-2 iterations. Fitting all four response functions simultaneously is advantageous because all terms of the cross section can be calculated directly including the corresponding uncertainties. However, this approach has limited sensitivity to the dependence on $\theta$.

This method of iterating the model cross section relies on two assumptions. 
First, it is assumed that the variation of the cross section is small and no modification to the functional form is necessary. Second, it is assumed that the angular coverage is dominated by high $\epsilon$ data having full $\phi$ coverage. The full angular acceptance is important in fitting the $\phi$-dependence and determining the interference terms of the cross section. The validity of this assumption can be tested by fitting the high and low $\epsilon$ data separately and comparing the results. Both methods were found to be in good agreement. The entire iteration procedure can be described in three steps: determining ratios of data and Monta Carlo yields, fitting of response functions in $(\phi, t)$ acceptance and calculation of experimental cross sections. The cross section fitting procedure is described in more detail below.

For the purpose of calculating the data and Monte Carlo ratios one dimensional histograms were created and filled with experimental and simulated yields binned in the variables in which the iteration is performed. In the $\mathrm{F}_{\pi}-2$ analysis these variables are $\phi$ and $t$. For each bin in $\phi$ and $t$ the ratio of the data and Monte Carlo distributions, $R(\phi, t)$, is calculated for each central angle setting. Given multiple kinematic settings for each $\epsilon$ setting there are at least two ways to combine these data into yield ratios. One could sum all experimental and all SIMC yields regardless of the angle setting and take the ratio: $R=Y_{\text {data }} / Y_{S I M C}$. On the other hand one could calculate the experimental to Monte Carlo ratios for each angle setting separately and combine the ratios in a weighted average using the standard formalism. Note that in both cases the ratios are calculated for each $(t, \phi)$ bin. As indicated above the second approach is used as the nominal calculation method in this analysis. Comparing both methods suggests no significant difference depending 
on the method. Additional histograms are created for $W$ and $Q^{2}$ for each bin in $t$ at each central angle setting and the average values over the phase space of the bin are determined.

After creating the histograms and calculating the ratios of experimental and simulated yields, the measured cross section is averaged over the experimental $\phi$ acceptance. The coefficients in the reduced pion electroproduction cross section $d \sigma / d t d \phi$ are the four virtual photon polarization states $\sigma_{L}, \sigma_{T}, \sigma_{L T}$ and $\sigma_{T T}$, where $L$ and $T$ denote the longitudinal and transverse polarizations of the photon at the vertex. At least two methods are available to determine these coeffiecients. The first method is effectively a simultaneous fit to all four parameters. This approach relies on the assumption that the fit to the interference terms is constrained by the high $\epsilon$ data providing full $\phi$ coverage. The second method consists of two steps. In the first step the interference terms $\left(\sigma_{L T}, \sigma_{T T}\right)$ and the coefficient of the combination $\sigma_{T}+\epsilon \sigma_{L}$ are determined from a simultaneous fit. Note that for this step only high $\epsilon$ data are used. The combination $\sigma_{T}+\epsilon \sigma_{L}$ for the low $\epsilon$ dataset is calculated using the fitted high $\epsilon$ interference coefficients. After the $\phi$ dependence has been fitted, the longitudinal and transverse coefficients of the cross section can be extracted from the known epsilon dependence and a fit to a straight line. The longitudinal cross section is the slope and the transverse piece is the intercept at the $\epsilon=0$ crossing. Comparing the result from both methods suggests that there is no significant difference between the two fitting methods in the longitudinal and transverse pieces.

At each iteration the experimental cross section is evaluated. Since the cross section model enters in both the calculation of the SIMC yield and the central 
model cross section this requires re-calculating both quantities at each step of the iteration. Note that the $\phi$ dependent terms in the cross section are re-calculated at each iteration as well. A representative example of the measured experimental cross section as a function of $\phi$ for a particular $t$-bin is shown in figure 5.2. The cosine structure from the interference terms is clearly visible. The two curves denote the fit result from a fit of the measured cross section to the $\phi$-dependence of the cross section in equation 1.20 .

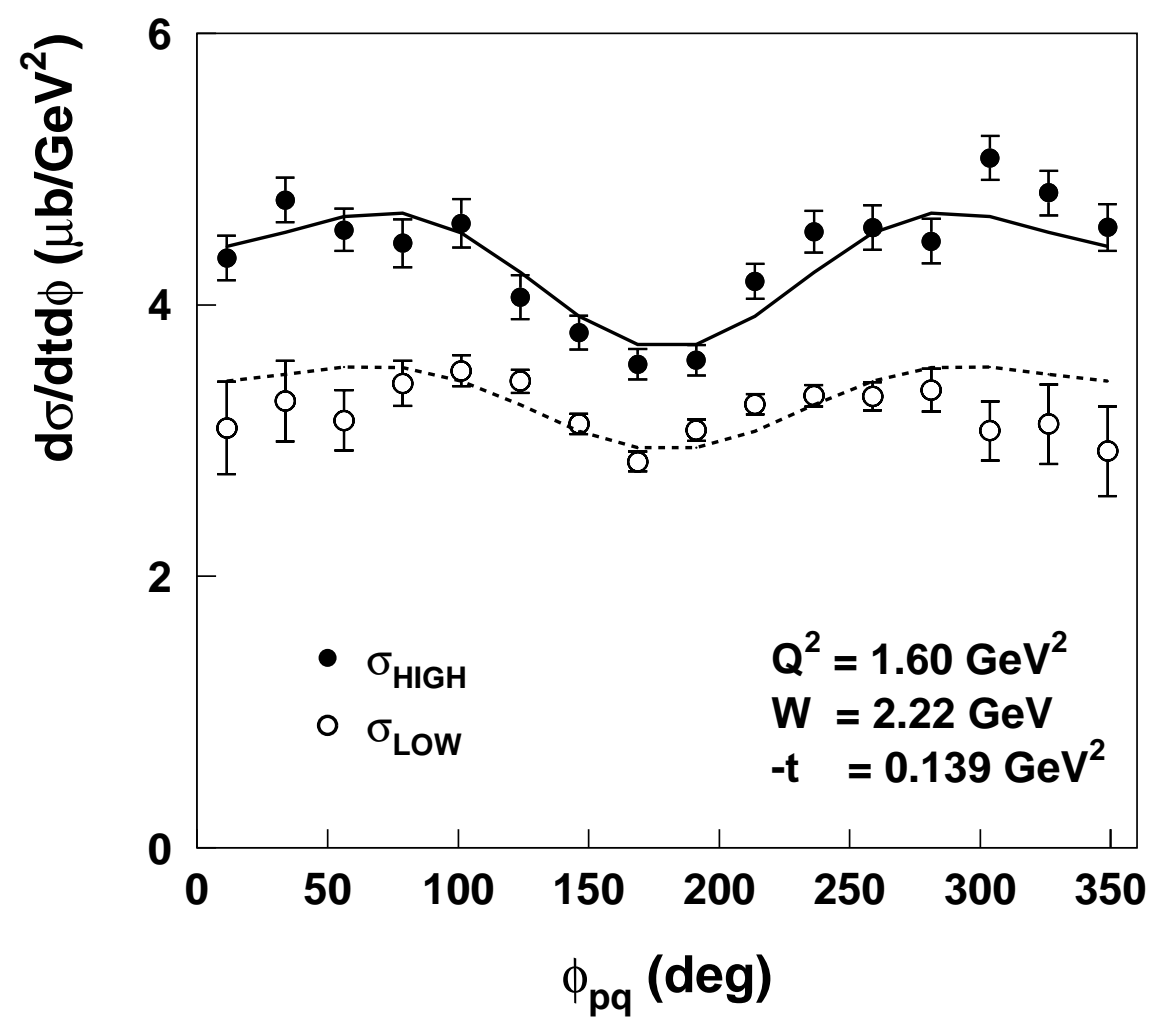

Figure 5.2: Representative plot of the experimental cross sections, $\frac{d^{2} \sigma}{d t d \phi}$ as a function of azimuthal angle $\phi$ at $Q^{2}=1.6(\mathrm{GeV} / \mathrm{c})^{2}$ for high and low $\epsilon$. The curves shown represent the fit of the measured values of the cross section to equation 1.20. In this fit all four parameters (the L,T,LT and TT response functions) are fitted simultaneously.

While generally valid, the iteration procedure described above may affect the 
description of kinematic variables in an adverse way. This is the case whenwever fitting is poorly constrained like at the edges of available phase space.

\subsubsection{Cross Section Model Dependence}

The starting model used in this analysis is based on a cross section parameterization developed during the $\mathrm{F}_{\pi}-1$ analysis. The model was modified for consistency with existing pion electroproduction and photoproduction data and applies to the dynamic range of this experiment. The parameterization is shown below.

$$
\begin{aligned}
\frac{d \sigma_{L}}{d t} & =A \frac{Q^{2}}{\left(1+1.77 Q^{2}+0.05 Q^{4}\right)^{2}} e^{\left(B-C \ln \left(Q^{2}\right)\right) \cdot(-t)} \\
\frac{d \sigma_{T}}{d t} & =\frac{D}{Q^{2}}+\frac{E}{Q^{4}} \\
\frac{d \sigma_{T T}}{d t} & =-\frac{F}{Q^{4}} \cdot \frac{|t|}{\left(|t|+m_{\pi}^{2}\right)^{2}} \cdot \sin \left(\theta^{*}\right)^{2} \\
\frac{d \sigma_{L T}}{d t} & =\left(e^{G-\frac{H}{\sqrt{Q^{2}}} \cdot t}+K-\frac{L}{Q^{4}}\right) \cdot \sin \left(\theta^{*}\right)
\end{aligned}
$$

where $A, B, C, D, E, F, G, H, K$ and $L$ are the fit parameters with values shown in Table 5.1. The $W$ dependence of the data in this model is assumed to follow the phase space factor $\left(W^{2}-M_{p}^{2}\right)^{-2}$. This function has the form of the $t$-pole term and has been used in previous analyses of electroproduction data described in references $[14,6]$. Since the $W$-dependence has not been fit explicitely, the validity of this assumption may be challenged. However, a comparison of experimental cross sections extracted at different values of $W$ for $\mathrm{F}_{\pi^{-}}-1(W=1.95)$ and $\mathrm{F}_{\pi^{-}}-2(W=2.22)$ suggests good agreement with the phase space factor prediction. Though limited to moderate values of $W$, this result gives confidence in the $W$-dependence of the 


\begin{tabular}{||c|c|c||}
\hline Parameter & Initial value & Final value \\
\hline \hline A & 350 & 214 \\
B & 16 & 8.6 \\
C & 7.5 & 0.7 \\
D & 4.5 & 3.9 \\
E & 2 & 5.8 \\
F & 5 & -14.9 \\
G & 0.79 & 3.98 \\
H & 3.4 & 0.8 \\
K & 1.1 & 0.7 \\
L & 3.6 & -22.5 \\
\hline
\end{tabular}

Table 5.1: Fit parameters for equation 5.9 before and after iterating the model. Convergence was achieved within 2 iterations.

model in the kinematic region of interest for this experiment. If the model describes the data adequately the distributions should show no significant discrepancy and the ratio in each kinematic variable is expected to be unity. Since the data are well described by this parameterization (see Figure 5.3) no major modifications are required to the initial parameterization. However, to test the influence of the functional form on the cross section extraction a different parameterization was also considered.

The model dependence and dependence on the iteration method are estimated from the difference in the extracted experimental cross sections between un-iterated and iterated cross sections. The value of the total uncertainty varies by $t$-bin between 0.6 and $4.2 \%$, where the largest uncertainty corresponds to large $-t$. The uncorrelated contribution was taken as the average model dependence between high 

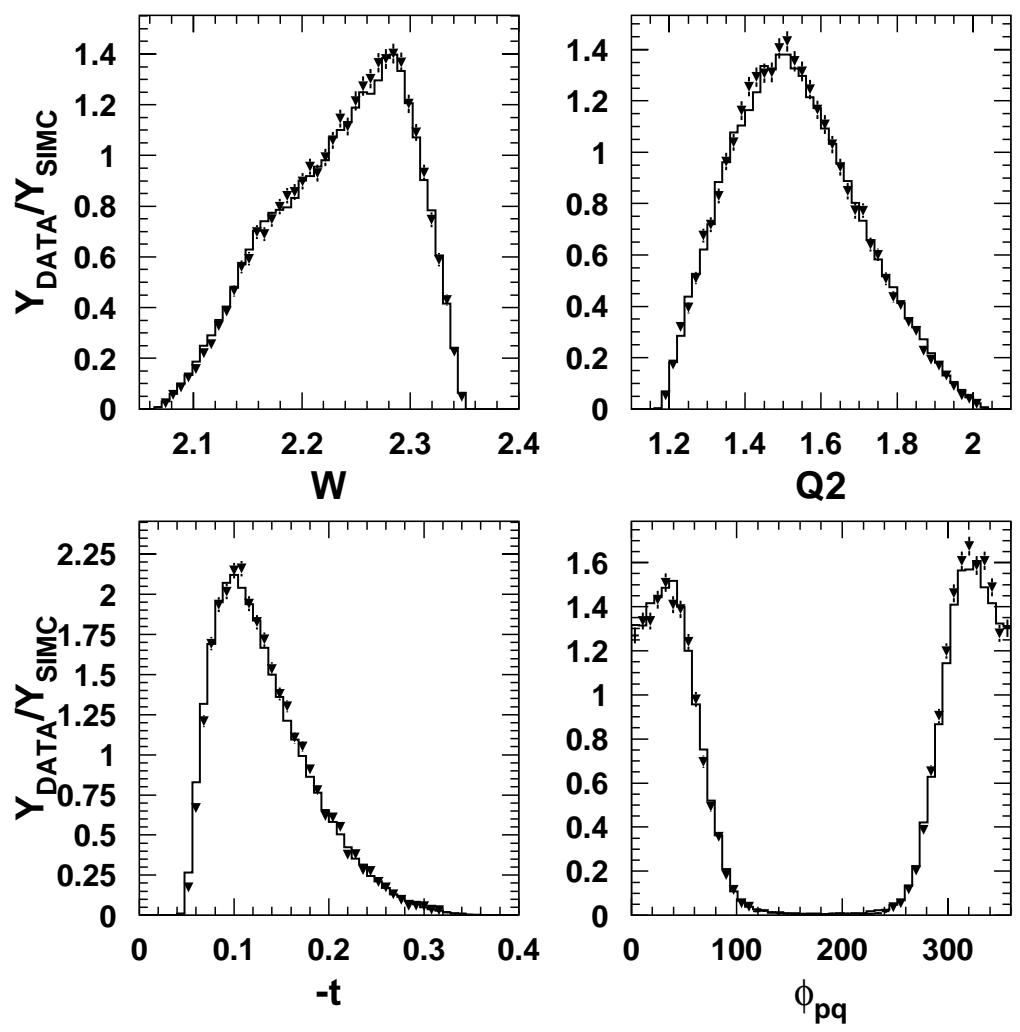

Figure 5.3: Comparison of Data (crossed) and SIMC (solid line) for the relevant pion electroproduction physics quantities, $W, Q^{2},-t, \phi$ and $\theta$. The Monte Carlo is weighted by the model input cross section. The distributions are normalized to each other by a global scale factor.

and low $\epsilon$ settings for all $t$-bins. The correlated piece of the uncertainty results then from the linear subtraction of correlated from the total uncertainty. The resulting uncorrelated contribution to the overall uncertainty varies between 0.1 and $2.2 \%$ between $t$-bins. As a further test of the model dependence, the experimental cross section was extracted using a "flat" model. This test entails an extreme modification to the model retaining only the $\epsilon$ and $\phi$ dependence of the model cross section. Since the iteration procedure assumes that the kinematic dependence is approximately accurate, this model is not a priori expected to provide a good description of the data. 
The flat model was iterated by first fitting a correction function to the data and adjusting the parameters according to the iteration procedure described above. The flat starting model also provides a way to estimate the model dependence itself. The iterated result at one iteration suggests a model dependence of $1.7-5.8 \%$ varying by t-bin relative to the nominal parameterization. The uncorrelated contribution is on the order of $2 \%$ for all $t$-bins.

The contribution of the interference terms, $\sigma_{L T}$ and $\sigma_{T T}$, to the systematic uncertainty is already included in the estimate of the model dependence of the cross section. To estimate the total contribution, the cross section was calculated two ways. In the first case the cross section was calculated by explicitly fitting the characteristic $\cos (2 \phi)$-dependence. In the second case the cross section was extracted by averaging over the experimental $\phi$ acceptance. The uncertainty due to the interference terms is then the difference between the two methods. The resulting uncertainty is on the order of $0.2 \%$.

\subsection{Cross Section Spectrometer Acceptance Dependence}

The proximity to the pion pole makes the experimental cross section very sensitive to kinematic offsets and correlations in the spectrometer acceptance at small values of $-t$. As a test of the general understanding of the spectrometer acceptance elastic data were studied. Figure 5.4 shows the experimental $y_{\text {tar }}^{\prime}$ acceptance for elastic $e-p$ data. The experimental spectrometer acceptance is relatively well described with the exception of a small region centered around $y_{t a r}^{\prime}=20 \mathrm{mrad}$. The contribution of events from the region where the discrepancy between data and 
Monte Carlo is most clearly observable to all events in this region is $6 \%$. A similar discrepancy in the HMS $y_{\text {tar }}^{\prime}$ is observed in pion electroproduction data from hydrogen.

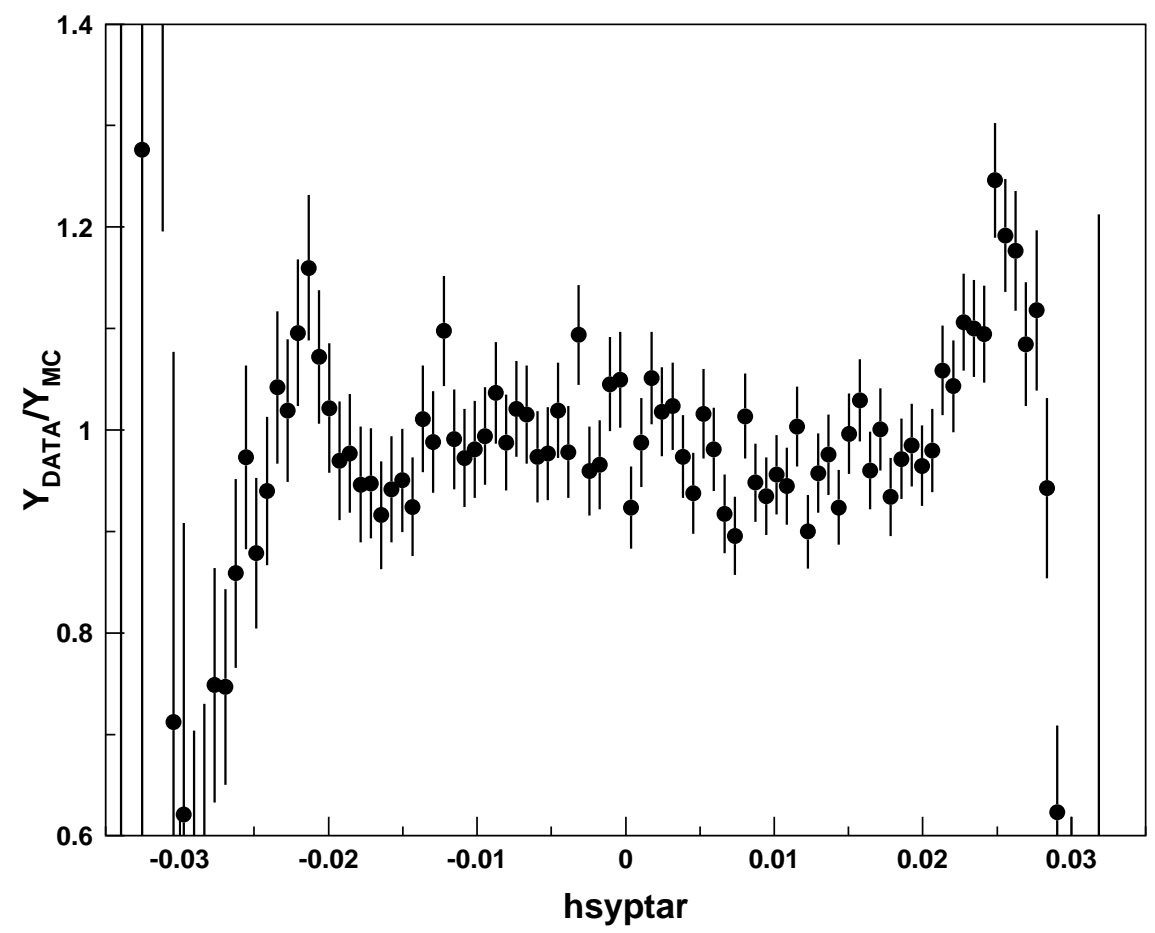

Figure 5.4: Ratio of data and simulated charge normalized yields across the $y_{\text {tar }}^{\prime}$ acceptance for elastic $e-p$ data at $P_{H M S}=3.83(\mathrm{GeV} / \mathrm{c})$ and $\theta_{H M S}=12^{\circ}$.

The dependence of the experimental cross section on spectrometer acceptance was tested by varying the cuts on the quantities $\left(\delta, x_{t a r}^{\prime}, y_{\text {tar }}^{\prime}\right)$ in each spectrometer. The experimental cross section was then extracted for spectrometer cut variations of $\pm 10 \%$ and compared to the one with nominals cuts. In general, the variation of the cross section is small $(<0.5 \%)$. However, the value of the cross section at small values of $-t$ depends significantly on the HMS $y_{\text {tar }}^{\prime}$ acceptance. The increased sensitivity at small $-t$ compared to other spectrometer quantities may be attributable to the strong correlation with HMS $y_{\text {tar }}^{\prime}$. 
Various aspects of the sensitivity of the cross section to spectrometer acceptance were investigated. For example, kinematic offsets and correlations play an important role in the spectrometer acceptance as well. As part of the spectrometer acceptance studies a dependence of HMS $\delta$ on $y_{f p}^{\prime}$ was eliminated as described in section 3.2.2. To exclude resolution effects in the data, HMS $y_{\text {tar }}^{\prime}$ matrix elements were optimized. Altough the result improves the agreement between data and SIMC, a significant discrepancy remains in the region 20-30 mrad.

A large part of the observed sensitivity can be attributed to resolution effects at the edges of the acceptance. The fraction of events contributing to the overall yields depends on the kinematic setting and the value of $-t$. Note that a comparatively large number of events are located in the region $20-30 \mathrm{mrad}$ at small $-t$. To correct for the resolution effects in the HMS $y_{\text {tar }}^{\prime}$ acceptance, a correction function was fit from $e-p$ data and was applied to the Monte Carlo yield on an event by event basis. The cross section extracted with the HMS $y_{\text {tar }}^{\prime}$ acceptance weight is in relatively good agreement with the one extracted eliminating the region of discrepancy using spectrometer cuts. Applying the acceptance correction function does not have a significant effect on any of other kinematic quantities.

The uncertainty in the experimental cross section due to the sensitivity to the HMS $y_{\text {tar }}^{\prime}$ acceptance is estimated from the fractional difference in cross sections extracted with and without cutting on the $y_{t a r}^{\prime}$ acceptance. The total uncertainty obtained by this method varies between 0.4 and $2.1 \%$ and has both correlated and uncorrelated parts, which also differ by $t$-bin. The common correlated uncertainty between $\epsilon$ points and the range in uncertainties covered by $t$-bin are listed in table 
5.2 along with a range of uncorrelated uncertainties between $t$-bins.

\subsection{Error Analysis}

The uncertainty in the extraction of the separated cross sections consists of both statistical and systematic uncertainties. The statistical uncertainty is determined by the uncertainty in the number of real good events. Even more important in an L-T separation are the two types of errors which determine the systematic uncertainty in the final cross section. Random, or point-to-point uncertainties affect each $\epsilon$ setting independently and are included at each $\epsilon$ point separately when extracting the experimental separated cross sections, $\sigma_{L}$ and $\sigma_{T}$. This is done by adding the random systematic uncertainty in quadrature to the statistical uncertainty:

$$
d \sigma=\sqrt{d \sigma_{\text {stat }}^{2}+d \sigma_{\text {random }}^{2}}
$$

Uncertainties that are the same at both $\epsilon$ points are called scale errors and are directly associated to the separated cross sections.

$$
\begin{aligned}
d \sigma_{L} & =\sqrt{\frac{d \sigma_{1}^{2}+d \sigma_{2}^{2}}{\left(\epsilon_{1}-\epsilon_{2}\right)^{2}}} \\
d \sigma_{T} & =\sqrt{\left(\frac{\epsilon_{1}}{\epsilon_{1}-\epsilon_{2}} \cdot d \sigma_{2}\right)^{2}+\left(\frac{\epsilon_{2}}{\epsilon_{1}-\epsilon_{2}} \cdot d \sigma_{1}\right)^{2}}
\end{aligned}
$$

where $\sigma_{1}$ and $\sigma_{2}$ denote the unseparated cross sections at high and low $\epsilon$ points. The largest scale errors include the uncertainty in the radiative correction method and the uncertainty in the pion absorption correction. The systematic uncertainties 
are summarized in table 5.2. The uncertainty in the cross section due to kinematic offsets (beam energy, central angles etc.) is estimated by varying each kinematic quantity in the electroproduction model cross section by its corresponding uncertainty. The error due to variation in the kinematic quantities is then the difference between the cross sections extracted with nominal and adjusted kinematic offsets. The typical uncertainties in beam energy and electron momenta are $3 \times 10^{-4}$ and $0.5 \times 10^{-3}$. The uncertainty in the spectrometer central angle is taken to be 0.5 mrad. The magnitude of the uncertainty due to the kinematic quantities depends on $-t$ and is largest at small values of $-t$. While some uncertainties are easily recognized as point-to-point between $\epsilon$ settings, they may be partially correlated when looking at the $t$-dependence at fixed $\epsilon$. This class of errors includes the uncertainties associated with kinematic offsets, because varying kinematic offsets may change the t-dependence of high (and low) $\epsilon$ unseparated cross sections in a correlated way at each $\epsilon$ point. These uncertainties are estimated from the variation in the extracted $F_{\pi}$ value when kinematic offsets are applied at high or low $\epsilon$ points. This is done by varying the unseparated cross sections separately by the corresponding $1 \sigma$ deviation of the kinematic offset from its mean value. For example, for a beam energy offset, $\delta\left(E_{e}\right)$, at high $\epsilon$ the error on the extracted longitudinal cross section, $\sigma_{L}$, can be written as

$$
\delta \sigma_{L}=\frac{\sigma_{1}\left(1+\delta\left(E_{e}\right)\right)-\sigma_{2}}{\epsilon_{1}-\epsilon_{2}}-\frac{\sigma_{1}-\sigma_{2}}{\epsilon_{1}-\epsilon_{2}}
$$

where $\sigma_{1}$ and $\sigma_{2}$ denote the high $\epsilon$ and low $\epsilon$ unseparated cross sections. Another class of uncertainties that cannot be easily categorized are dependent on particle 
rates or beam currents. If the rates or currents were exactly the same between $\epsilon$ points the nature of the uncertainty would be entirely scale. This is the case for the target density correction. However, if there are significant fluctuations in both settings the contribution of random uncertainties cannot be neglected. Specific examples of these uncertainties include electronic dead time and HMS Čerenkov blocking correction. These uncertainties are estimated varying the unseparated cross sections simultaneously by the corresponding $1 \sigma$ deviation of the rate dependent correction from the mean value for a particular kinematic setting. 


\begin{tabular}{|c|c|c|c|c|}
\hline Correction & $\begin{array}{c}\text { Point-to-point } \\
(\%)\end{array}$ & $\begin{array}{c}\text { Scale } \\
(\%)\end{array}$ & $\begin{array}{c}\text { Correlated (-t) } \\
(\%)\end{array}$ & Section \\
\hline HMS Aerogel & & & 0.04 & 3.4 .3 \\
\hline SOS Cer & & & 0.02 & 3.4 .2 \\
\hline SOS Cal & & & 0.17 & 3.4 .2 \\
\hline HMS beta & & & 0.09 & \\
\hline Charge & & 0.5 & 0.3 & \\
\hline Target Thickness & & 0.8 & 0.2 & 2.6 \\
\hline HMS Tracking & & 1.0 & 0.4 & 3.5 .2 \\
\hline SOS Tracking & & 0.5 & 0.1 & \\
\hline $\mathrm{CPU}$ & & & 0.2 & 3.5 .3 \\
\hline SOS coincidence block. & & & 0.1 & 3.6 \\
\hline HMS Trigger & & & 0.1 & 3.5 .1 \\
\hline SOS Trigger & & & 0.1 & \\
\hline Pion absorption & & 2.0 & 0.1 & 3.8 \\
\hline Al cell wall & & & 0.01 & 3.4 .4 \\
\hline Ele DT & & & 0.1 & 3.5 .3 \\
\hline HMS Cer block & & & 0.1 & 3.7 \\
\hline$\theta_{e}$ & 0.1 & & $0.7-1.1$ & \\
\hline$E_{\text {beam }}$ & 0.1 & & $0.2-0.3$ & 5.6 \\
\hline$p_{e}$ & 0.1 & & $0.1-0.3$ & \\
\hline$\theta_{\pi}$ & 0.1 & & $0.2-0.3$ & \\
\hline Radiative corr & & 2.0 & 0.4 & 4.6 \\
\hline Two-photon & & 0.5 & 0.5 & \\
\hline Acceptance & $1.0(0.6)$ & 1.0 & 0.6 & 5.5 \\
\hline Pion decay & 0.03 & 1.0 & & 4.5 \\
\hline Model Dep & 0.2 & 0.5 & $1.1-1.3$ & 5.4 .2 \\
\hline
\end{tabular}

Table 5.2: Summary of systematic uncertainties. The uncertainties listed correspond to the uncertainty in the cross section as a result of the uncertainty in the correction. Point to point uncertainites are applied at each $\epsilon$ point when calculating the separated cross sections, $\sigma_{L}$ and $\sigma_{T}$. Scale uncertatinties are directly associated to the separated cross sections. The values in the "Correlated (-t)" column denote uncertainties that change the t-dependence of the high and low epsilon dependent cross sections in a correlated way. These uncertainties are applied directly to $F_{\pi}$ after extracting $\Lambda_{\pi}^{2}$. Adding all uncertainties in quadrature results in a total point to point uncertainty of 1.2 (0.9)\% and a total scale uncertainty of 3.5\%. The partially correlated uncertainty ranges between $1.8 \%$ and $1.9 \%$. 
Chapter 6

Results and Discussion

\subsection{Overview}

The goal of $\mathrm{F}_{\pi}-2$ is to isolate the longitudinal part of the pion electroproduction cross section for the purpose of extracting $F_{\pi}$ assuming pion pole dominance. In the subsequent sections, both unseparated and separated cross section and the extracted values for $F_{\pi}$ are presented.

In order to interpret the cross section results the measured cross sections are compared to a Regge model by Vanderhaeghen, Guidal and Laget [11]. The main focus is on the longitudinal term of the cross section, which is dominated by the pion exchange process. This process can be described as quasifree scattering of a virtual pion. The longitudinal cross section is related to the product of $F_{\pi}$, the probability for a virtual photon to interact with a pion, and $g_{\pi N N}$, the probability for the nucleon to emit a virtual pion. The value for $F_{\pi}$ is extracted by comparing the values of the experimental longitudinal cross section to the result of a Regge calculation. The size of the transverse piece of the cross section is discussed in the context of a comparison between a Regge and a constituent quark model calculation. 


\subsection{Experimental Cross Sections}

The unpolarized pion electroproduction cross section in the laboratory frame can be expressed as,

$$
\frac{d^{5} \sigma}{d \Omega_{e} d E_{e}^{\prime} d \Omega_{\pi}}=J\left(t, \phi \rightarrow \Omega_{\pi}\right) \Gamma_{v} \frac{d^{2} \sigma}{d t d \phi_{\pi}}
$$

where $J\left(t, \phi \rightarrow \Omega_{\pi}\right)$ is the Jacobian transforming the hadronic part of the differential virtual photon cross section and

$$
\Gamma_{v}=\frac{\alpha}{2 \pi^{2}} \frac{E_{e}^{\prime}}{E_{e}} \frac{1}{Q^{2}} \frac{1}{1-\epsilon} K
$$

is the virtual photon flux. $\alpha$ is the fine structure constant, $\epsilon$ is the longitudinal polarization of the virtual photon, and $K$ is the equivalent real photon energy in the Hand convention,

$$
K=\frac{W^{2}-M^{2}}{2 M}
$$

where $M$ is the target mass and $W$ is the invariant mass of the photon-nucleon system. The unpolarized electroproduction cross section can be written in terms of the four polarization states of the virtual photon, the longitudinal, transverse and two interference terms.

$$
2 \pi \frac{d \sigma}{d t d \phi}=\frac{d \sigma_{T}}{d t d \phi}+\epsilon \frac{d \sigma_{L}}{d t d \phi}+\sqrt{2 \epsilon(1+\epsilon)} \frac{d \sigma_{L T}}{d t d \phi} \cos \phi_{\pi}+\epsilon \frac{d \sigma_{T T}}{d t d \phi} \cos 2 \phi_{\pi}
$$


The four response functions are extracted by fitting the entire experimental $\phi$ acceptance.

\subsection{Unseparated Cross Sections}

The unseparated experimental cross sections are extracted as discussed in

Chapter 5 . The cross sections, $\frac{d \sigma}{d t}$, for both $\epsilon$ points are binned in $-t$, the momentum transfer to the nucleon. The cross sections are evaluated at the center of each $t$-bin with the corresponding average values of $W$ and $Q^{2}$ for that bin. The experimental

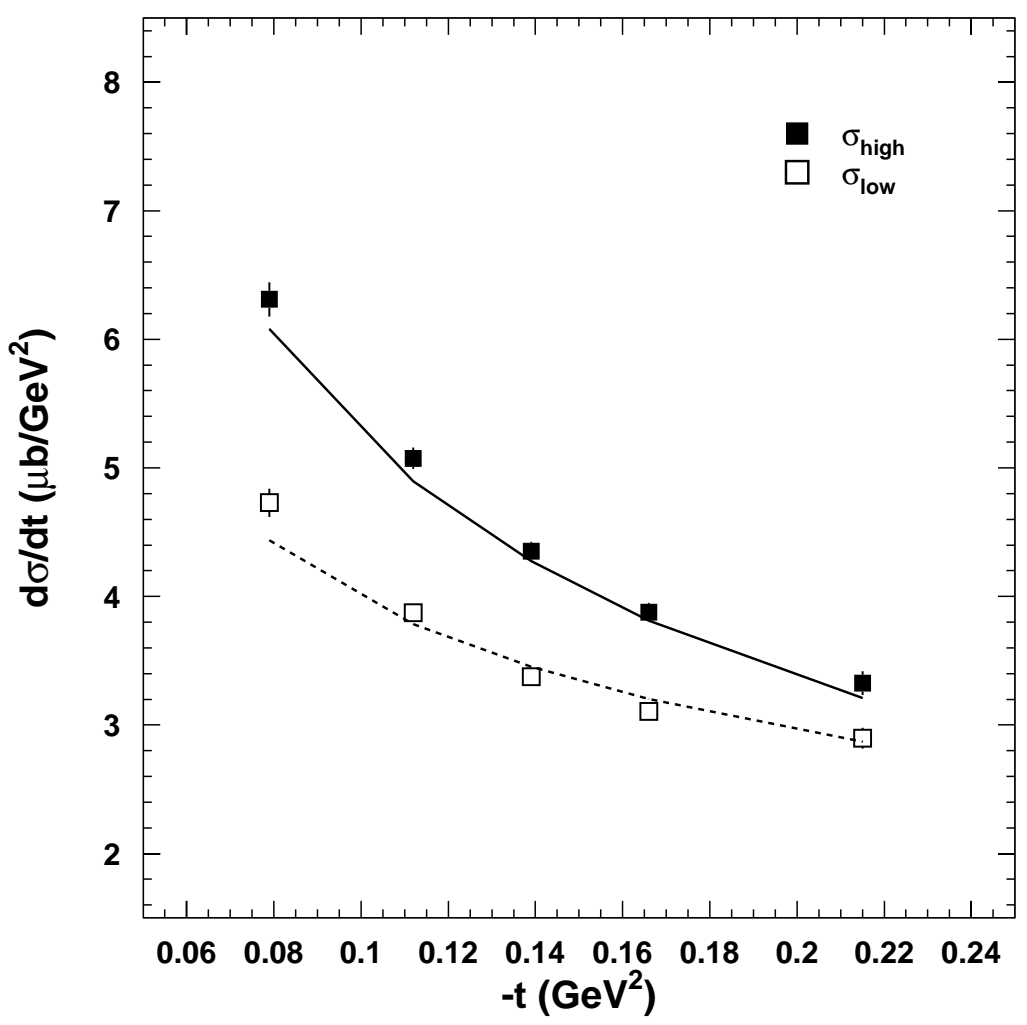

Figure 6.1: Experimental unseparated cross sections at $Q^{2}=1.60(\mathrm{GeV} / \mathrm{c})^{2}$. The solid and dashed lines indicate the model prediction for hgigh and low $\epsilon$ respectively.

and model unseparated cross sections are shown in Figure 6.1. The model cross 


\begin{tabular}{||c|c|c|c|c|c|c|c||}
\hline $\begin{array}{c}Q^{2} \\
\left(\mathrm{GeV}^{2}\right)\end{array}$ & $\begin{array}{c}W \\
(\mathrm{GeV})\end{array}$ & $\begin{array}{c}t \\
\left(\mathrm{GeV}^{2}\right)\end{array}$ & $\begin{array}{c}\theta^{*} \\
(\mathrm{deg})\end{array}$ & $\epsilon_{\text {low }}$ & $\begin{array}{c}\sigma_{\text {low }} \\
\left(\mu \mathrm{b} / \mathrm{GeV}^{2}\right)\end{array}$ & $\epsilon_{\text {high }}$ & $\begin{array}{c}\sigma_{\text {high }} \\
\left(\mu \mathrm{b} / \mathrm{GeV}^{2}\right)\end{array}$ \\
\hline \hline 2.274 & 1.416 & 0.079 & 5.36 & 0.313 & $4.701 \pm 0.10$ & 0.585 & $6.344 \pm 0.11$ \\
2.242 & 1.513 & 0.112 & 9.14 & 0.320 & $3.890 \pm 0.07$ & 0.589 & $5.089 \pm 0.09$ \\
2.213 & 1.593 & 0.139 & 11.19 & 0.329 & $3.401 \pm 0.06$ & 0.593 & $4.371 \pm 0.08$ \\
2.187 & 1.667 & 0.166 & 12.94 & 0.335 & $3.122 \pm 0.06$ & 0.597 & $3.902 \pm 0.07$ \\
2.153 & 1.763 & 0.215 & 16.04 & 0.343 & $2.929 \pm 0.06$ & 0.602 & $3.351 \pm 0.07$ \\
\hline \hline 2.308 & 2.215 & 0.145 & 6.61 & 0.254 & $2.163 \pm 0.04$ & 0.545 & $2.768 \pm 0.05$ \\
2.264 & 2.279 & 0.202 & 10.77 & 0.260 & $1.749 \pm 0.03$ & 0.548 & $2.143 \pm 0.03$ \\
2.223 & 2.411 & 0.245 & 12.58 & 0.267 & $1.598 \pm 0.03$ & 0.552 & $1.878 \pm 0.03$ \\
2.181 & 2.539 & 0.288 & 13.96 & 0.275 & $1.520 \pm 0.03$ & 0.557 & $1.742 \pm 0.03$ \\
2.127 & 2.703 & 0.365 & 16.82 & 0.285 & $1.401 \pm 0.03$ & 0.563 & $1.558 \pm 0.03$ \\
\hline
\end{tabular}

Table 6.1: Unseparated cross sections, $\frac{d \sigma}{d t}$, for the $\left(e, e^{\prime}, \pi^{+}\right)$reaction. The results are presented in the lab frame for the center value of each $t$ bin. The values for $W$ and $Q^{2}$ are the weighted averages for each $t$ bin averaged over the high and low $\epsilon$ points. The values for $\epsilon$ and $\theta^{*}$ are calculated from $W, Q^{2}$ and $t$ in each such bin and the corresponding beam energies for the $\epsilon$ calculation. The uncertainties shown are statistical and random systematic combined in quadrature. Note that random systematic uncertainties have to be included at each $\epsilon$ point using equation 6.6, while scale systematic uncertainties propagate directly into the separated cross sections.

section is illustrated as solid and dashed lines for high and low $\epsilon$ cross sections.

\subsection{Separated Cross Sections}

After eliminating the two interference terms the virtual photon cross section can be expressed as

$$
2 \pi \frac{d \sigma}{d t}=\frac{d \sigma_{T}}{d t}+\epsilon \frac{d \sigma_{L}}{d t}
$$

where the interference terms have been determined from a fit to the $\phi$ dependence at large $\epsilon$. The longitudinal and transverse terms of the cross section can then be extracted by varying the photon polarization, $\epsilon$, and fitting a straight line to the known $\epsilon$ dependence. The longitudinal cross section is given by the slope and the 
transverse cross section by the $(\epsilon=0)$ intercept. The separated cross sections can be written,

$$
\begin{aligned}
\frac{d \sigma_{L}}{d t} & =\frac{\sigma_{1}-\sigma_{2}}{\epsilon_{1}-\epsilon_{2}} \\
\frac{d \sigma_{T}}{d t} & =\frac{\epsilon_{2} \sigma_{1}-\epsilon_{1} \sigma_{2}}{\epsilon_{1}-\epsilon 2}
\end{aligned}
$$

where $\sigma_{1}$ and $\sigma_{2}$ are the unseparated high and low $\epsilon$ cross sections.

Alternatively, all four response functions can be determined simultaneously by fitting the entire experimental $\phi$ acceptance. The results from both methods agree within the uncertainty of the cross sections. Table 6.2 lists the separated cross sections, $\sigma_{T}$ and $\sigma_{L}$, extracted using equation 6.6. Though not the main focus in this experiment, the size of the separated transverse cross sections may be of general interest. While the Regge model predicts a negligible transverse component of the cross section independent of $Q^{2}, \mathrm{~F}_{\pi}-2$ data suggest an approximately equal contribution at $Q^{2}=2.45(\mathrm{GeV} / \mathrm{c})^{2}$. To address this issue, $\mathrm{F}_{\pi}-2$ data set may be used to constrain current models of the pion electroproduction process.

In order to extract the value of $F_{\pi}$, the longitudinal cross section is compared to the results of a Regge calculation by Vanderhaeghen, Guidal and Laget. This Regge model does a good job in describing the separated cross sections of previous L-T separated data such as the DESY, and $\mathrm{F}_{\pi^{-}}$-1 data. However, it should be noted that the model under-estimates $\sigma_{T}$ consistently at all values of $Q^{2}$. The VGL model calculations for $\mathrm{F}_{\pi}-2$ longitudinal and transverse cross sections are shown in Fig 6.2. The $\pi$ and $\rho$ trajectories are included in this calculation. The $\pi$ trajectory 


\begin{tabular}{||c|c|c|c|c|c|c|}
\hline $\begin{array}{c}Q^{2} \\
(\mathrm{GeV} / \mathrm{c})^{2}\end{array}$ & $\begin{array}{c}W \\
(\mathrm{GeV})\end{array}$ & $\begin{array}{c}t \\
\left(\mathrm{GeV}^{2}\right)\end{array}$ & $\begin{array}{c}\theta^{*} \\
(\mathrm{deg})\end{array}$ & $\begin{array}{c}\sigma_{L} \\
\left(\mu \mathrm{b} / \mathrm{GeV}^{2}\right)\end{array}$ & $\begin{array}{c}\sigma_{T} \\
\left(\mu \mathrm{b} / \mathrm{GeV}^{2}\right)\end{array}$ & $\mathrm{R}=\frac{\sigma_{L}}{\sigma_{T}}$ \\
\hline \hline 2.274 & 1.416 & 0.079 & 5.358 & $6.060 \pm 0.60$ & $2.802 \pm 0.27$ & $2.16 \pm 0.30$ \\
2.242 & 1.513 & 0.112 & 9.137 & $4.470 \pm 0.46$ & $2.459 \pm 0.21$ & $1.81 \pm 0.24$ \\
2.213 & 1.593 & 0.139 & 11.19 & $3.661 \pm 0.40$ & $2.198 \pm 0.19$ & $1.67 \pm 0.23$ \\
2.187 & 1.667 & 0.166 & 12.94 & $2.975 \pm 0.37$ & $2.124 \pm 0.18$ & $1.39 \pm 0.21$ \\
2.153 & 1.763 & 0.215 & 16.04 & $1.630 \pm 0.35$ & $2.369 \pm 0.19$ & $0.69 \pm 0.16$ \\
\hline \hline 2.308 & 2.215 & 0.145 & 6.61 & $2.078 \pm 0.23$ & $1.635 \pm 0.11$ & $1.27 \pm 0.16$ \\
2.264 & 2.279 & 0.202 & 10.77 & $1.365 \pm 0.16$ & $1.395 \pm 0.08$ & $0.98 \pm 0.13$ \\
2.223 & 2.411 & 0.245 & 12.58 & $0.980 \pm 0.14$ & $1.337 \pm 0.08$ & $0.74 \pm 0.11$ \\
2.181 & 2.539 & 0.288 & 13.96 & $0.786 \pm 0.14$ & $1.304 \pm 0.08$ & $0.60 \pm 0.11$ \\
2.127 & 2.703 & 0.365 & 16.82 & $0.564 \pm 0.16$ & $1.240 \pm 0.08$ & $0.46 \pm 0.12$ \\
\hline
\end{tabular}

Table 6.2: Separated cross sections for the $\left(e, e^{\prime}, \pi^{+}\right)$reaction for both values of $Q^{2}$. The errors on the cross sections are statistical and systematic, while the errors on the ratios are the total errors.

is parameterized in a monopole form,

$$
F_{\pi}\left(Q^{2}\right)=\frac{1}{1+\frac{Q^{2}}{\Lambda_{\pi}^{2}}}
$$

where $\Lambda_{\pi}^{2}$ denotes the corresponding cutoff parameter $\Lambda_{\pi}^{2}$. Since most of the free parameters in the Regge model have been fixed by photoproduction data, the value of $F_{\pi}$ can be obtained from a one-parameter fit to equation 6.7 . The value of $\Lambda_{\pi}^{2}$ is determined from a comparison of the longitudinal experimental cross section and the result of the Regge calculation at each value of $Q^{2}$. The values for the cutoff parameters shown in Figure 6.2 are $\Lambda_{\pi}^{2}=0.512(0.491) \mathrm{GeV}^{2}$ at values of $Q^{2}=1.60$ (2.45) $(\mathrm{GeV} / \mathrm{c})^{2}$. The values of $\Lambda_{\rho}^{2}$ were chosen to be 0.6 and $2.1 \mathrm{GeV}^{2}$ to illustrate the sensitivity of the longitudinal and transverse cross sections to the $\rho$ trajectory. The $\rho$ exchange has little influence on $\sigma_{L}$ and ranges between 0.5 and $1 \%(3 \%)$ at $Q^{2}=1.60(2.45) \mathrm{GeV}^{2}$ over the experimental acceptance. The transverse cross section 
varies by $13 \%(30 \%)$ at $Q^{2}=1.60(2.45)(\mathrm{GeV} / \mathrm{c})^{2}$ at small values of $-t$. Finally, the value for $F_{\pi}$ is extracted from a one-parameter fit with $\Lambda_{\pi}^{2}$ at the minimum of the $\chi^{2} /$ dof distribution (see Figure 6.4).
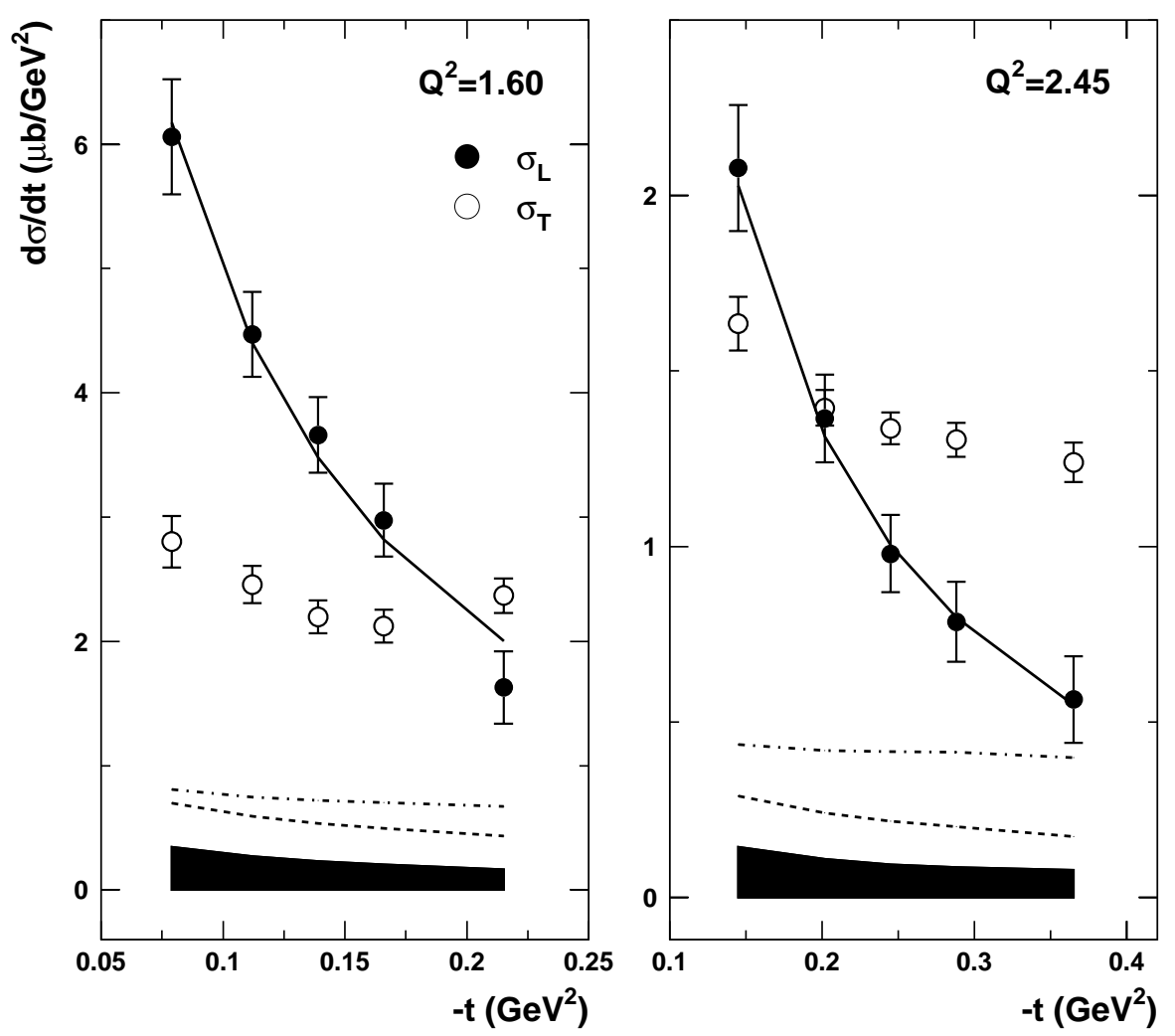

Figure 6.2: Experimental separated cross sections, $\sigma_{L}$ and $\sigma_{T}$ compared to $V G L$ Regge calculations with values of $\Lambda_{\pi}^{2}=0.512$ (0.491) $\mathrm{GeV}^{2}$ at values of $Q^{2}=1.60$ (2.45) $(\mathrm{GeV} / \mathrm{c})^{2}$. The error bars indicate statistical and random systematic uncertainty combined in quadrature. The error band denotes the correlated part of the systematic uncertainty by which all data points move collectively. The dashed line and dashed-dotted lines indicate VGL Regge calculations for $\sigma_{T}$ for values of $\Lambda_{\rho}^{2}$ of 0.6 and $2.1(\mathrm{GeV} / \mathrm{c})^{2}$.

The VGL model compares well with the separated longitudinal cross section, $\sigma_{L}$. However, the transverse cross sections are systematically underpredicted by 
more than a factor of two. Note that the same trend was observed in the analysis of $\mathrm{F}_{\pi^{-}}-1$ data. This is likely due to the choice of parameters used to describe the $\rho N N$ vertex in the VGL model calculation. The model is based on the Bonn potential, but does not include all parameters in the case of the strong $\rho N N$ vertex. Instead of the monopole parameterization of the strong $\rho N N$ form factor, a t-dependent form of the Regge parameterization was used. This likely results in the loss of total strength in the $\rho N N$ vertex, which may be directly observable in the strength of the transverse cross section.
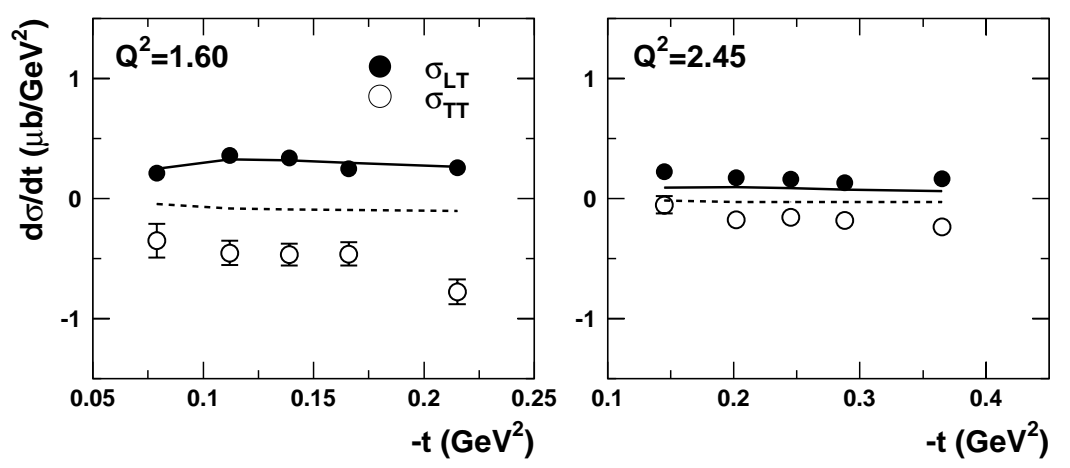

Figure 6.3: Experimental separated cross sections, $\sigma_{L T}$ and $\sigma_{T T}$ obtained from a 4 parameter fit to the experimental $\phi$ dependence. The error bars are statistical only. The solid and dashed lines represent the VGL Regge model results for $\sigma_{L T}$ and $\sigma_{T T}$ with $\Lambda_{\pi}^{2}=0.512(\mathrm{GeV} / \mathrm{c})^{2}$ and $\Lambda_{\pi}^{2}=0.491(\mathrm{GeV} / \mathrm{c})^{2}$.

The interference terms shown in Figure 6.3 have been fitted explicitly from the data using a fit to the experimental $\phi$ dependence. The values of $\sigma_{T T}$ and $\sigma_{L T}$ are compared to those of the VGL model ${ }^{1}$. To estimate the $W$-dependence of the interference terms, $\sigma_{L T}$ and $\sigma_{T T}$ were evaluated in the VGL Regge model for $\mathrm{F}_{\pi}-1$ and $\mathrm{F}_{\pi}-2$ kinematics at the same values of $Q^{2}$ and $-t$. The shape of

\footnotetext{
${ }^{1}$ The sign convention used for $\sigma_{T T}$ is the one from reference [11]. Thus, the sign of the experimental cross section is inverted.
} 
the $\sigma_{L T}$ is generally reproduced correctly at all values of $Q^{2}$, but the model cross section is generally lower relative to the data. The absolute value of $\sigma_{T T}$ calculated in the VGL model underestimates the data. This is likely in agreement with the observation of the VGL model underpredicting the strength of the transverse cross section, since the same currents enter in the calculations. Thus, if the strength of $\sigma_{T}$ is underpredicted the strength of $\sigma_{T T}$ may be underpredicted as well. Note that $\sigma_{L T}$ may be influenced as well.

\subsection{Extraction of $F_{\pi}$}

For the purpose of extracting $F_{\pi}$ from experimental data, the results of VGL/Regge calculations were compared to experimental cross sections. The VGL/Regge model has been shown to be consistent with existing pion photo- and electroproduction data for values of $W>2 \mathrm{GeV}$. The VGL/Regge model compares well with $\sigma_{L}$, but there is clearly room for improvement for $\sigma_{T}, \sigma_{T T}$ and $\sigma_{L T}$. However, assuming that the pion exchange process dominates at small values of $-t$, the contribution of other mechanisms is expected to be small.

The presence of the $\rho$ trajectory does not significantly influence $\sigma_{L}$ and in turn the extraction of $F_{\pi}$. The dependence on the $\rho$ trajectory was studied by varying the $\Lambda_{\rho}^{2}$ parameter in the Regge calculation. The value of $\sigma_{L}$ did not deviate significantly from its nominal value. However, a large influence on the transverse piece of the cross section can be observed.

Values for $F_{\pi}$ were extracted using a $\chi^{2}$ optimization of the experimental value for $\sigma_{L}$ to the VGL model prediction (see Figure 6.4). The uncertainty in $\Lambda_{\pi}^{2}$ can be 


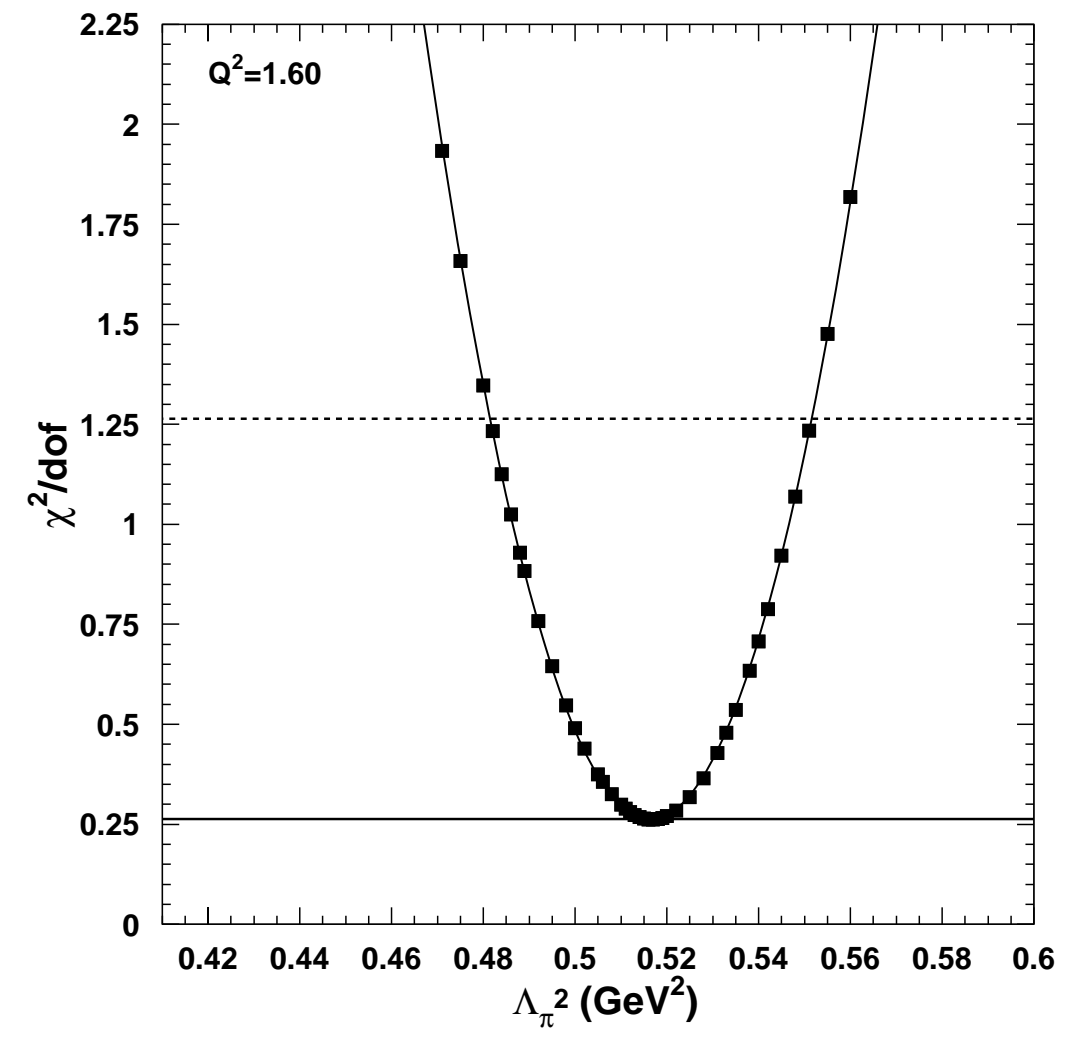

Figure 6.4: $\chi^{2}$ distribution for a comparison of $\sigma_{L}$ from VGL/Regge calculations and $F_{\pi}$-2 experimental data. The data are fit with a parabola and the value of $F_{\pi}$ is extracted at the minimum of the $\chi^{2}$ distribution (solid line). The dashed line indicates the uncertainty in $\Lambda_{\pi}^{2}$ when $\chi^{2}$ varies by one standard deviation.

expressed in terms of the curvature of the $\chi^{2}$ function in the region of the minimum. For a standard parabola, $\chi^{2}\left(\Lambda_{\pi}^{2}\right)=a+b\left(\Lambda_{\pi}^{2}-c\right)^{2}$, the uncertainty in the parameter $\Lambda_{\pi}^{2}$ can be written [110],

$$
\sigma_{\Lambda_{\pi}^{2}}=\sqrt{2\left(\frac{d \chi^{2}}{d \Lambda_{\pi}^{2}}\right)^{-1}}
$$

where $\left(\frac{d \chi^{2}}{d \Lambda_{\pi}^{2}}\right)=\frac{1}{\sqrt{b}}$ and $b$ is determined from the fit parabola. Using equation 6.7, the corresponding uncertainty in $F_{\pi}$ can be expressed as the first derivative with 


\begin{tabular}{||c|c|c|c|c|c||}
\hline $\begin{array}{c}Q^{2} \\
(\mathrm{GeV} / \mathrm{c})^{2}\end{array}$ & $F_{\pi}$ & $\begin{array}{c}\left(\frac{d F_{\pi}}{F_{\pi}}\right)^{\text {random }} \\
(\%)\end{array}$ & $\begin{array}{c}\left(\frac{d F_{\pi}}{F_{\pi}}\right)^{\text {scale }} \\
(\%)\end{array}$ & $\begin{array}{c}\left(\frac{d F_{\pi}}{F_{\pi}}\right)^{t-c o r r} \\
(\%)\end{array}$ & $\begin{array}{c}\left(\frac{d F_{\pi}}{F_{\pi}}\right)^{\text {total }} \\
(\%)\end{array}$ \\
\hline \hline 1.60 & 0.243 & 4.98 & 1.74 & 0.50 & 5.30 \\
2.45 & 0.167 & 6.25 & 1.54 & 0.52 & 6.46 \\
\hline
\end{tabular}

Table 6.3: Values for $\Lambda_{\pi}^{2}$ and $F_{\pi}$. The values for $\Lambda_{\pi}^{2}$ are extracted from a comparison of the separated longitudinal cross section to the one from the VGL/Regge model prediction and $F_{\pi}$ is determined using equation 6.7. Note that these values are dependent on the particular model used.

respect to $\Lambda_{\pi}^{2}$,

$$
\frac{F_{\pi}}{\Lambda_{\pi}^{2}}=-\left(\frac{Q^{2}}{\Lambda_{\pi}^{4}}\right) \frac{-1}{\left(1+\frac{Q^{2}}{\Lambda_{\pi}^{2}}\right)^{2}},
$$

where $Q^{2}$ is the central value for each setting and $\Lambda_{\pi}^{2}$ is the cutoff at the minimum of the $\chi^{2}$ distribution. Alternatively, the uncertainty in $F_{\pi}$ due to the experimental uncertainty can be determined by calculating $\Lambda_{\pi}^{2}$ at one standard deviation from the minimum value of $\chi^{2}$.

The VGL/Regge model describes the experimental data well, so that no further modifications to the model are necessary. The resulting values for $F_{\pi}$ are listed in Table 6.3. Figure 6.5 shows the values for $F_{\pi}$ from this analysis compared to values from DESY, and Jefferson Lab $\mathrm{F}_{\pi}-1$. The JLab data taken at $Q^{2}=1.60(\mathrm{GeV} / \mathrm{c})^{2}$ were taken at different values of $W$ to allow for studying the model dependence in the extraction of $F_{\pi}$. The extraction depends necessarily on a model of the $\mathrm{p}\left(e, e^{\prime} \pi^{+}\right) \mathrm{n}$ reaction. However, comparing measurements at different proximity to the pion pole allows for a direct statement on the model dependence. $\mathrm{F}_{\pi^{-}}-1$ and $\mathrm{F}_{\pi^{-}}$-2 data are in good agreement suggesting a small model dependence in the extrapolation to small $-t$ 


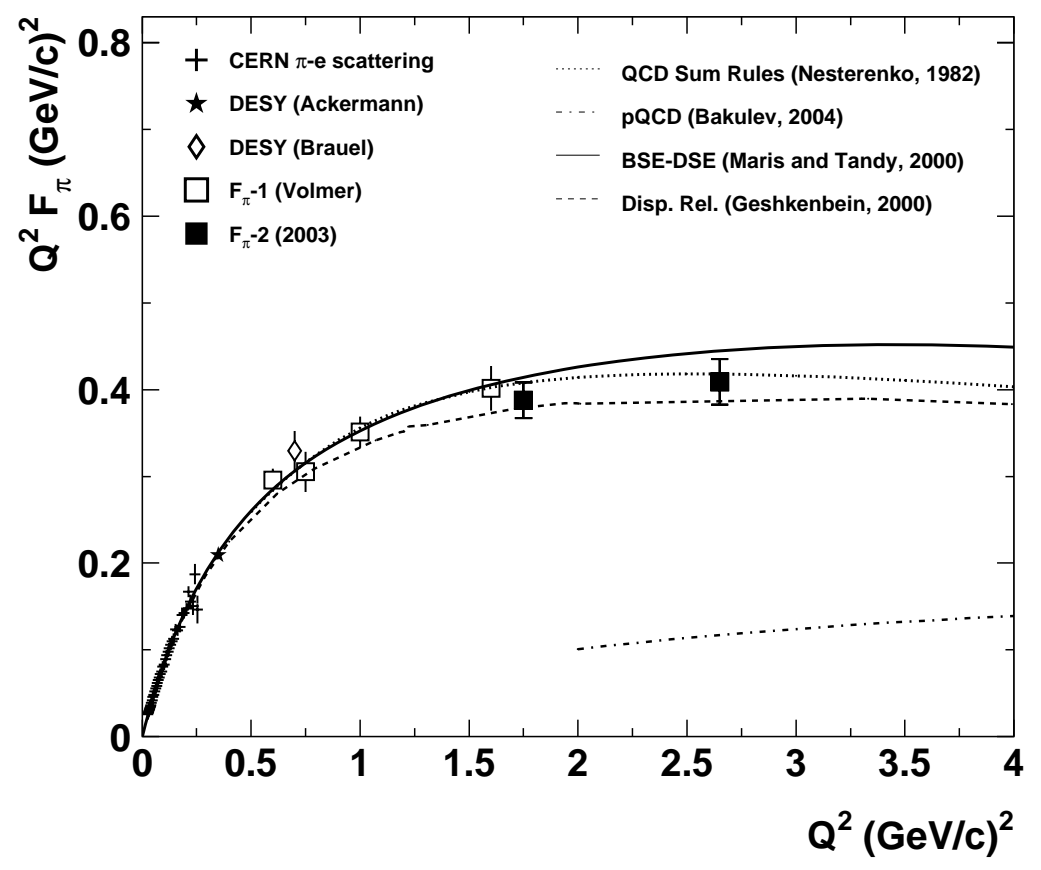

Figure 6.5: Pion form factor from $F_{\pi}$-2 compared to data from CERN, DESY and JLab $F_{\pi}-1$. The error bars combine the statistical and experimental systematic uncertainties in quadrature. The curves are from a Dyson-Schwinger equation (dasheddotted), QCD sum rules (dashed) and from a $p Q C D$ calculation (dotted). All models describe the data well up to values of $Q^{2}=1.60(\mathrm{GeV} / \mathrm{c})^{2}$. The $F_{\pi}$-2 data point at $Q^{2}=1.60(\mathrm{GeV} / \mathrm{c})^{2}$ deviates by $\approx 1 \sigma$ from the Dyson Schwinger calculation by Maris and Tandy.

\section{$6.6 \pi^{-} / \pi^{+}$Ratio and Pole Dominance}

The extraction of $F_{\pi}$ from the electroproduction longitudinal cross section relies on the dominance of the pion exchange process. To test pole term dominance for $\mathrm{F}_{\pi^{-}} 2$ data experimentally, pion electroproduction from deuterium is used. This entails taking data at both positive and negative polarity. The dominance of the pole term is then studied through the ratio of longitudinal $\pi^{-}$and $\pi^{+}$cross sections, which should be identical. The ratio of longitudinal cross sections can be written in 
terms of the isovector and isoscalar amplitudes,

$$
\frac{\sigma\left(\gamma^{*} n \rightarrow \pi^{-} p\right)}{\sigma\left(\gamma^{*} p \rightarrow \pi^{+} n\right)}=\frac{\left|A_{v}-A_{s}\right|^{2}}{\left|A_{v}+A_{s}\right|^{2}}
$$

The pion exchange process is purely isovector, so that a ratio of unity suggests the absence or suppression of isoscalar background. It should be noted that the amplitudes in equation 6.10 are complex. Particular combinations of relative phases in the ratio may therefore still be consistent with unity and effectively hide the contribution of isoscalar processes. Though the ratio of longitudinal cross sections may be consistent with unity the interpretation of deviations in terms of isoscalar contributions is nontrivial.

The pion electroproduction cross section from deuterium is complicated by an extra degree of freedom and depends on six kinematic quantities instead of five in the hydrogen case. The lab cross section can be written,

$$
\frac{d^{6} \sigma}{d \Omega_{e} d E_{e}^{\prime} d \Omega_{\pi} d P_{\pi}}=\Gamma\left(\frac{d \sigma}{d \Omega_{\pi} d P_{\pi}}\right)
$$

and the virtual photon cross section can be separated into longitudinal, transverse and interference term contributions,

$\frac{d \sigma}{d \Omega_{\pi} d P_{\pi}}=2 \pi \frac{d \sigma_{T}}{d \Omega_{\pi} d P_{\pi}}+\epsilon \frac{d \sigma_{L}}{d \Omega_{\pi} d P_{\pi}}+\sqrt{2 \epsilon(1+\epsilon)} \frac{d \sigma_{L T}}{d \Omega_{\pi} d P_{\pi}} \cos \phi_{\pi}+\epsilon \frac{d \sigma_{T T}}{d \Omega_{\pi} d P_{\pi}} \cos 2 \phi_{\pi}$

The analysis of the data was done analogous to the analysis of hydrogen data 

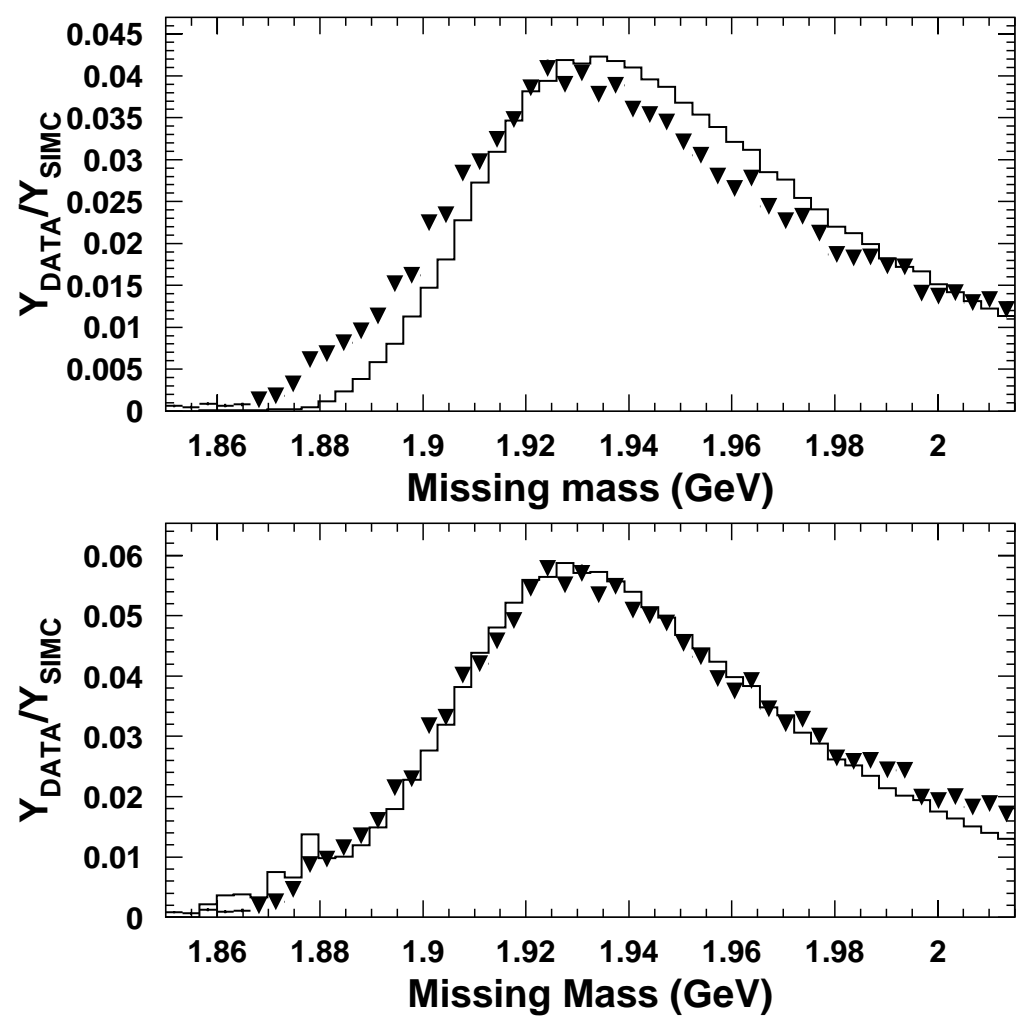

Figure 6.6: Missing mass distribution for $\pi^{+}$production from deuterium. Triangles indicate experimental data and the solid line is the Monte Carlo assuming quasifree production. The disagreement between data and Monte Carlo may be attributed to not taking into account $N N$ final states interactions in the Monte Carlo.

with the exception of the calculation of the model central cross section. In the Monte Carlo, pion production from deuterium and other nuclear targets is considered in the quasifree approximation taking the produced pion as a particle with momentum $p_{F}$. In the quasifree approximation, interactions between the recoiling nucleons in the final state ( $N N$ force or Pauli exclusion) are neglected. The effect of $N N$ final state interactions is illustrated in Figure 6.6. At small relative momentum between the recoiling nucleons, $k_{r e l}$, the $N N$ force is attractive resulting in distortions to the shape of the missing mass distributions. 
The effects of he $N N$ force were included using the Jost function formalism where factorization from the dominant pion electroproduction process is assumed. In this case the electroproduction cross section is modified by the invserse of the magnitude squared of the Jost function,

$$
\sigma_{\pi}^{N N F S I}=\frac{1}{\left|J_{l}\left(k_{r e l}\right)\right|^{2}} \sigma_{\pi}
$$

where $J_{l}\left(k_{r e l}\right)$ is the Jost function for the $l$ th partial wave. In this analysis, only the lowest $S$ waves are of interest. The correction approaches unity as the $N N$ potential goes to zero, thus $k_{\text {rel }}$ becomes large. The $S$ wave Jost function can be written,

$$
J_{l}\left(k_{r e l}\right)=\frac{k_{r e l}-i \beta}{k_{r e l}-i \alpha}
$$

where $\alpha$ and $\beta$ are determined from experimental data for the scattering length, $a$, and the effective range, $r_{e}$, of the potential

$$
\begin{aligned}
\frac{1}{2} r_{e}(\alpha-\beta) & =1 \\
\frac{1}{2} r_{e} \alpha \beta & =-\frac{1}{a} .
\end{aligned}
$$

The functional form of the Jost function is implemented in the Monte Carlo similar as done in reference [55], but including the ${ }^{3} S_{0}$ interaction in addition to the ${ }^{1} S_{0}$ interaction. The scattering length and effective range for $\pi^{-}$(where the recoil system consists of two protons) were taken from proton-proton scattering data and the 
values for $\pi^{+}$(where the recoil system consists of two neutrons) were taken from neutron-neutron data. For the triplet the values from references $[111,112]$ were used. Including the triplet state does not significantly improve the result. The Jost function was further corrected by an ad-hoc correction to account for nontrivial contributions from Couloumb effects.

The central cross section was calculated in SIMC using a "point spectrometer" method. The electron spectrometer kinematics are determined from the average values of $Q^{2}$ and $W$ for each such bin for which the cross section is to be calculated. In this approach the cross section is averaged over the out-of-plane angle and the generation volume in the Monte Carlo is limited to a single point in angle and momentum. The pion spectrometer kinematics are specified for parallel kinematics with the spectrometer angle along the $\mathbf{q}$ vector. The angular generation volume is then used to select the central angle relative to $\mathbf{q}$. Processes like radiative effects, multiple scattering, energy loss and single arm spectrometer acceptance are disabled leaving only the event generation routines. The central cross section can be expressed,

$$
\left(\int \frac{d^{6} \sigma}{d \Omega_{e} d E_{e} d \Omega_{\pi} d P_{\pi}} d P_{\pi}\right)^{\text {central }}=\sum_{i} \frac{\Delta<\sigma_{\text {model }}^{5}>_{i}}{\Delta P_{\pi}} \cdot \Delta P_{\pi}
$$

where $\Delta<\sigma_{\text {model }}^{5}>_{i}$ is the five-fold cross section averaged over the nucleon wave function in the $i$ th $P_{\pi}$ bin. The experimental cross section is then extracted using equation 5.1. The results are shown in Figure 6.7 and Table 6.4.

The preliminary analysis of the longitudinal $\pi^{-}$to $\pi^{+}$ratios suggests relatively 


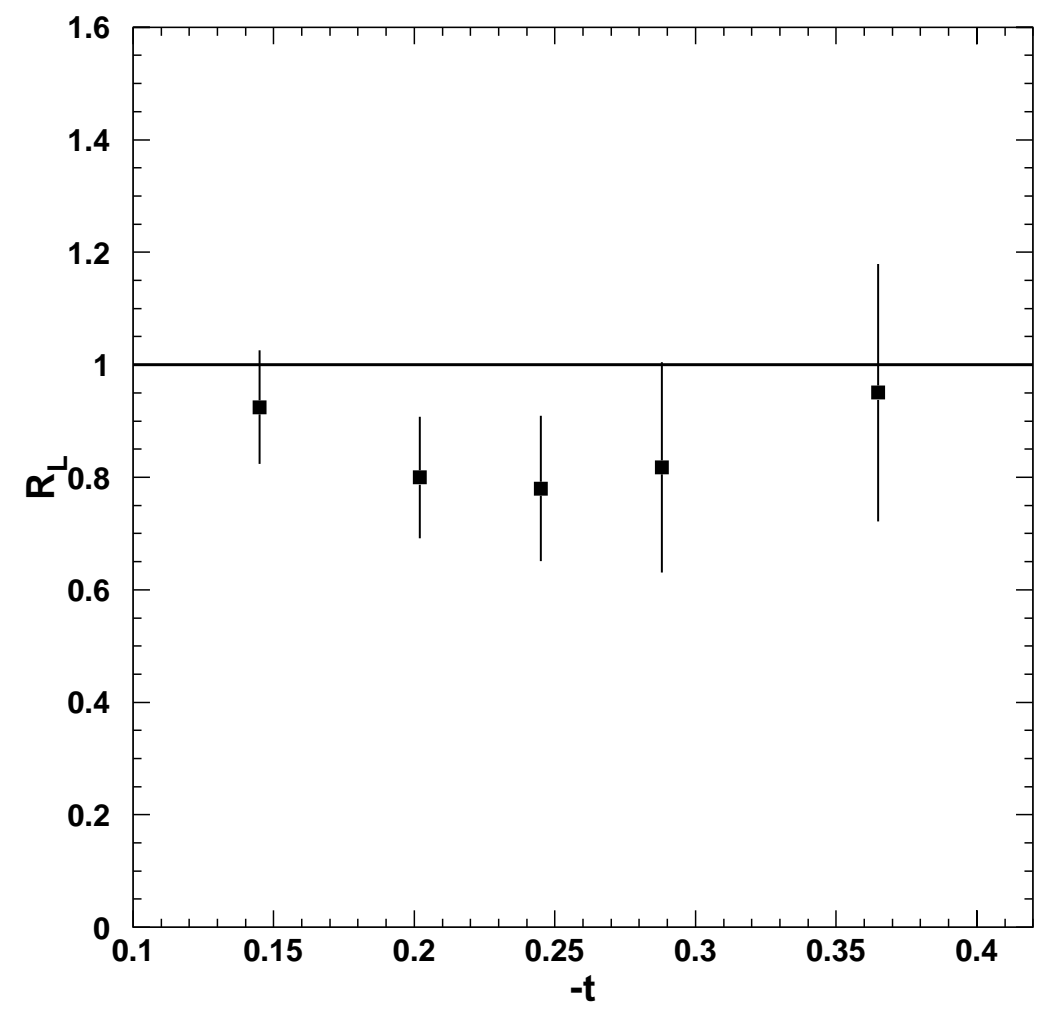

Figure 6.7: Longitudinal $\pi^{-} / \pi^{+}$ratios. The error bars on the data points are statistical and systematic.

good agreement with unity within the experimental uncertainty. Further analysis will include studies of the sensitivity of quantities such as the tracking efficiency on high rates, which may reduce the systematic uncertainty. In order to determine the size of contributions from isoscalar backgounds at the pion pole, the result from $\mathrm{F}_{\pi^{-}}-2$ will be combined with the four data points from $\mathrm{F}_{\pi^{-}}-1$ yielding a range in $Q^{2}$ from 0.6 to $2.45(\mathrm{GeV} / \mathrm{c})^{2}$. The experimental results for the longitudinal $\pi^{-}$to $\pi^{+}$ ratios can then be compared to the ones from the VGL/Regge model allowing for a prediction of the size of the background at the physical pion pole. 


\begin{tabular}{||l|c|c|c|c|c|c||}
\hline $\begin{array}{l}Q^{2} \\
(\mathrm{GeV} / \mathrm{c})^{2}\end{array}$ & $\begin{array}{c}W \\
(\mathrm{GeV})\end{array}$ & $\begin{array}{c}t \\
\left(\mathrm{GeV}^{2}\right)\end{array}$ & $\begin{array}{c}\theta^{*} \\
(\mathrm{deg})\end{array}$ & $R_{L}$ & $\delta R_{L}^{\text {stat }}$ & $\delta R_{L}^{\text {tot }}$ \\
\hline \hline 2.308 & 2.215 & 0.145 & 6.61 & 0.92 & 0.09 & 0.11 \\
2.264 & 2.279 & 0.202 & 10.77 & 0.80 & 0.09 & 0.11 \\
2.223 & 2.411 & 0.245 & 12.58 & 0.78 & 0.10 & 0.11 \\
2.181 & 2.539 & 0.288 & 13.96 & 0.88 & 0.14 & 0.16 \\
2.127 & 2.703 & 0.365 & 16.82 & 0.95 & 0.25 & 0.27 \\
\hline
\end{tabular}

Table 6.4: Longitudinal separated ratios cross section ratios. Statistical and total systematic uncertainties are shown. The cross sections have been evaluated using the hydrogen model.

\subsection{Results and Discussion}

Though the value of the pion form factor is still far from its asymptotic value at the values of $Q^{2}$ considered in this experiment, much can be learned from the most recent measurement of the pion form factor. Recall that pertubative QCD still underpredicts the data from previous measurements and that model predictions are not well constrained at moderate and higher values of $Q^{2}$. The $F_{\pi}$ result from $\mathrm{F}_{\pi}-2$ is shown in Figure 6.5 compared to different theoretical predictions discussed in Chapter 1. All models shown reproduce the pion charge radius up to values of $Q^{2}=1.60$ $(\mathrm{GeV} / \mathrm{c})^{2}$. At intermediate values of $Q^{2}$ all models reach a maximum value, depending on the separation scale included in the model. For example, the separation scale between quarks and gluon (soft) background in the constituent quark model is typically set at $Q^{2}=1(\mathrm{GeV} / \mathrm{c})^{2}$. Up to values of $Q^{2}$ of $1.60(\mathrm{GeV} / \mathrm{c})^{2}$, the data follow the monopole prediction for a pion charge radius $\Lambda_{\pi}^{2}=0.54(\mathrm{GeV} / \mathrm{c})^{2}($ Figure 6.8). Jefferson Lab data at $Q^{2}=2.45(\mathrm{GeV} / \mathrm{c})^{2}$ suggest a $1 \sigma$ deviation from the monopole form. The JLab $\mathrm{F}_{\pi}-2$ data indicate no turning point in $F_{\pi}$ up to $Q^{2}=2.45$ $(\mathrm{GeV} / \mathrm{c})^{2}$, but may indicate that a maximum value has been reached. The data are 
in relatively good agreement with a QCD sum rule calculation by Nesterenko and Radyushkin, who use a QCD sum rule framework for the soft contribution to $F_{\pi}$ as well as an asymptotically dominant hard gluon exchange term. The data also agree with the dispersion relation calculation by Geshkenbein. Dispersion relations relate timelike and spacelike regions of the pion form factor in the complex plane and the approach is generally rigorous, but not well constrained in the timelike region. The data are also reasonably well described by the Dyson-Schwinger calculation by Maris and Tandy, which is based on the Bethe-Salpeter equation with dressed quark and gluon propagators. All parameters are determined without the use of $F_{\pi}$ data $[24,48]$. The discrepancy at higher values of $Q^{2}$ may be attributable to not including all pion scattering diagrams in the calculation. Modifications to the BSE model which should make it more applicable at higher Q2 are currently underway [113]. While the focus of this experiment is on the longitudinal term of the pion electroproduction cross section and $F_{\pi}$, valuable information may be extracted from the separated transverse part of the cross section and the explicitly fitted interference terms. As illustrated in Figure 6.2, the VGL/Regge model underpredicts the transverse cross section. This may indicate that the strength of the $\pi \rho \gamma$ vertex is not described suitably in this electroproduction model. Further studies may focus on the feasability of obtaining information on the $F_{\pi \rho \gamma}$ transition form factor through the transverse pion electroproduction cross section. Note that the data were taken at a value of $W$ above the resonance region, so that background contribution may be excluded.

The longitudinal $\pi^{-} / \pi^{+}$ratios determined in this experiment indicate that the 


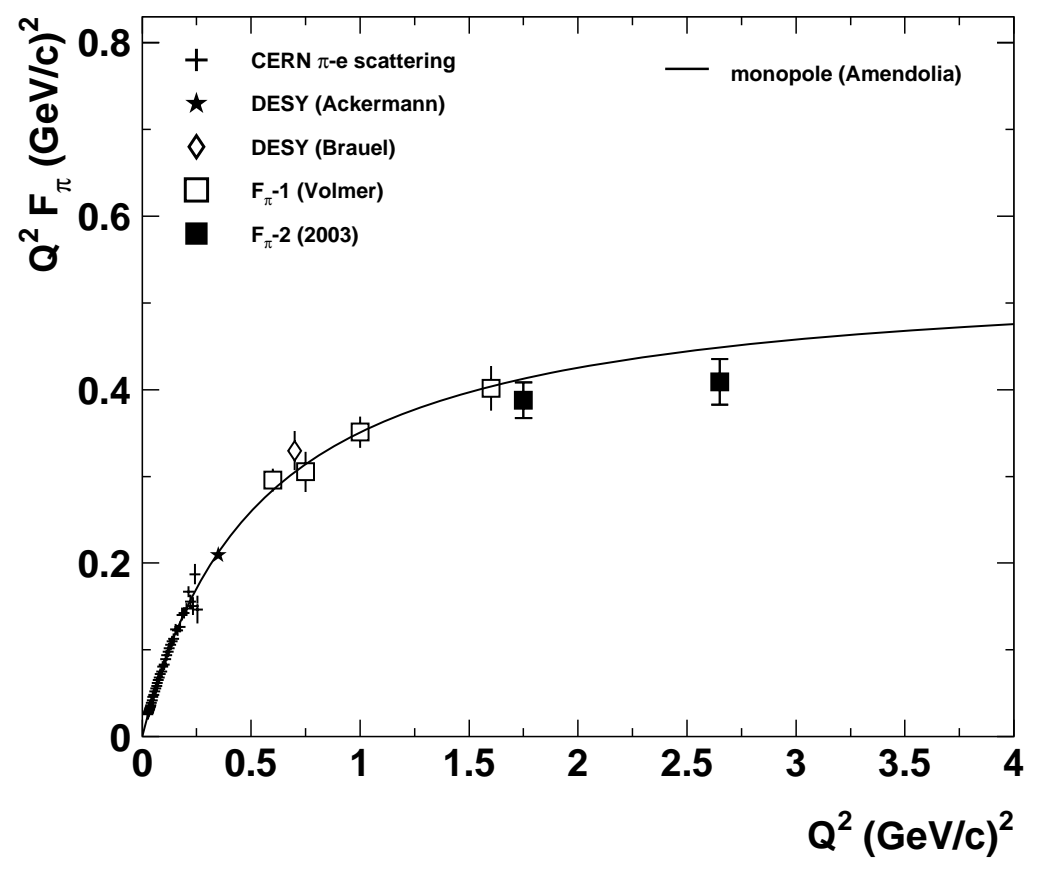

Figure 6.8: Pion form factor from $F_{\pi}$-2 compared to $e-p$ scattering data from CERN, and precision pion electroproduction from DESY and JLab $F_{\pi}-1$. The solid curve is a monopole fit to the CERN data [4]. The monopole prediction describes the data well up to $Q^{2}=1.60(\mathrm{GeV} / \mathrm{c})^{2}$. The $F_{\pi}$-2 data point at $Q^{2}=2.45(\mathrm{GeV} / \mathrm{c})^{2}$ deviates by $1 \sigma$ from the monopole form.

pion pole process is largely dominant at the kinematics of this experiment at small -t. However, no statement on the background contribution at the pion pole can be made at this point. Further analysis of thebackgrounds at small $t$ will combine the results from $\mathrm{F}_{\pi^{-}}-1$ and $\mathrm{F}_{\pi^{-}}-2$ and compare to the Regge model prediction.

\subsection{Future Measurements}

One of the fundamental challenges in nuclear physics is the description of hadrons in terms of the constituents of QCD. An important issue is the understand- 
ing of the transition region from long distance scales (low $Q^{2}$ ) to short distance scales (high $Q^{2}$ ). The simplest hadronic system available for study in its ground state is the pion, and the measurement of the pion form factor is the way to observe this transition experimentally. The most accurate data set for $F_{\pi}$ up to $Q^{2}=2.45$ $(\mathrm{GeV} / \mathrm{c})^{2}$ has been obtained in Hall $\mathrm{C}$ at JLab and it may be speculated that the maximum in the $Q^{2} F_{\pi}$ distribution has been reached. Future measurements are in preparation to extend the measurement of the pion form factor to a kinematic regime where one might begin to approach pQCD expectations. Recent theoretical studies indicate that hard exclusive processes contribute significantly at values of $Q^{2} \approx 5(\mathrm{GeV} / \mathrm{c})^{2}$

The JLab $12 \mathrm{GeV}$ upgraded electron beam allows for reliable $F_{\pi}$ measurements up to $Q^{2}=6(\mathrm{GeV} / \mathrm{c})^{2}$. In order to maximize the contribution of the pion pole to the cross section and to suppress non-pole backgrounds, $W$ has to be increased as well. Higher values of $W$ at fixed $Q^{2}$ allow for data taking at a smaller minimum values of $-t$, thus, reducing the distance to the pion pole. The optimal range was determined to be centered at $W=3 \mathrm{GeV}$. As in previous $F_{\pi}$ measurements, the proposed measurement will need full $\phi$ coverage for range of values of $-t$ to allow for the separation of the four response terms of the pion electroproduction cross section. Previously, full $\phi$ coverage could be obtained up to the point where longitudinal and transverse components become comparable. This occurs at $-t \approx 0.6(\mathrm{GeV} / \mathrm{c})^{2}$ for $Q^{2}=6(\mathrm{GeV} / \mathrm{c})^{2}$ kinematics. In the proposed measurement, pions will be emitted at very forward lab angles limiting the choice of the required apparatus. A good understanding of the spectrometer optics and kinematic offsets are essential. A 
recent review of the proposed spectrometers for the $12 \mathrm{GeV}$ uprade at JLab identified the Hall C HMS/SHMS combination as the most suitable detector setup. The design of the SHMS foresees a vertical bend, point-to-point focusing spectrometer similar to the HMS specifications. Two tunes will be available (Small Solid Angle and Large Solid Angle) of which the Small Angle Tune will allow for $F_{\pi}$ measurement at the required minimum angle of $5.5^{\circ}$. The rigid pivot design will ensure reproducibility of the pointing offsets and allow for reliable optics fitting at all angles over the full momentum range. In addition, the design goal momentum resolution will constrain the momentum offset. The acceptance will be designed flat to reduce systematic uncertaintied associated with the variation of rates. A detailed description of the proposed Hall C spectrometer systems at $12 \mathrm{GeV}$ can be found in reference [114]. In the proposed measurement, pions will be detected by the SHMS spectrometer and the coincident electrons will be detected in the HMS. Full $\phi$ coverage can be obtained in three angle settings of the SHMS. In parallel to the $F_{\pi}$ measurement, studies of the kinematic dependence of the pion electroproduction cross section are worth pursuing. For example, measurements at different values of $W$ could aid in constraining the $W$ dependence of the pion electroproduction cross section, which may further reduce the model uncertainty in $F_{\pi}$. Additional data on separated longitudinal and transverse cross sections may provide a constraint on the size of the transverse term in pion electroproduction models like the VGL/Regge model. The measurement of the transverse cross section may be of particular interest, since it may yield information on the pion transition form factor. 


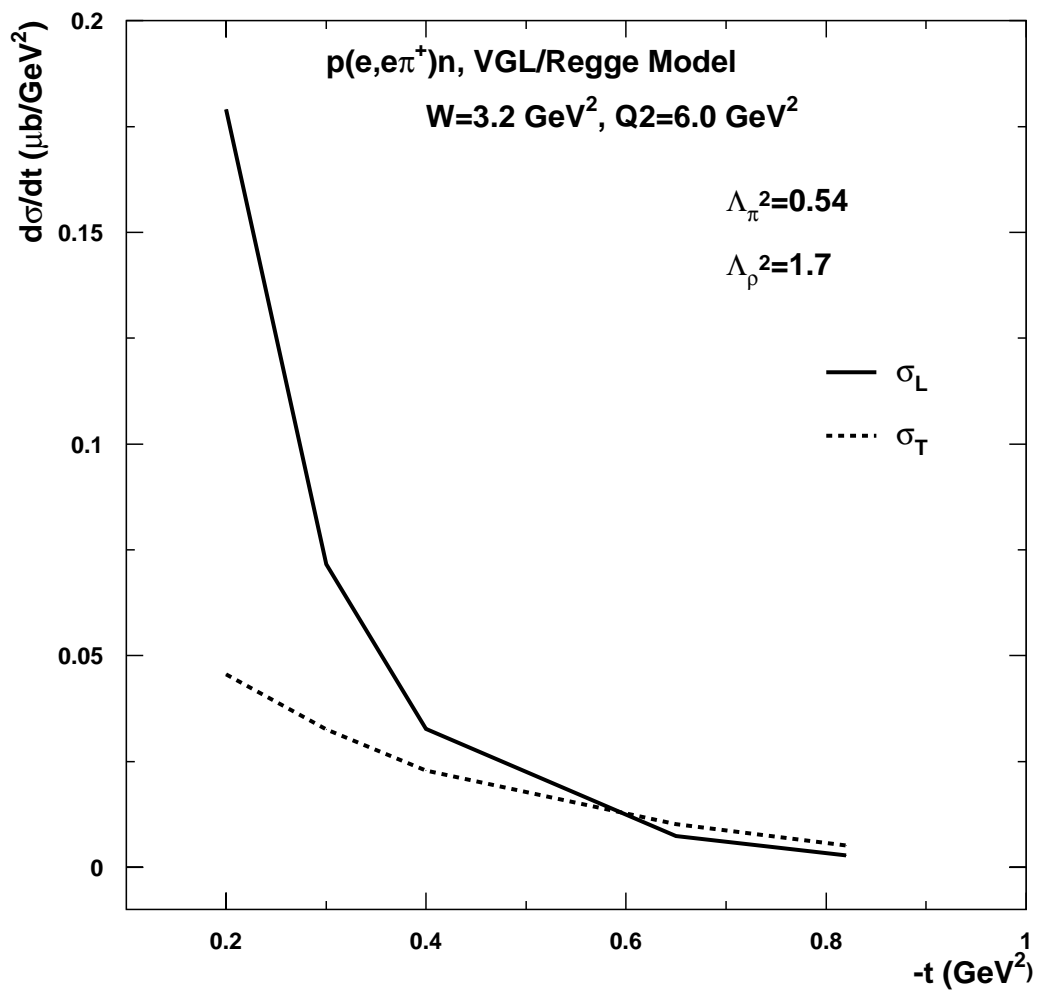

Figure 6.9: Pion electroproduction cross sections at 11 GeV kinematics. The longitudinal and transverse terms become comparable at $-t=0.6(\mathrm{GeV} / \mathrm{c})^{2}$.

\subsection{Conclusions}

The $\mathrm{F}_{\pi}-2$ experiment measured the longitudinal and transverse cross sections via pion electroproduction from hydrogen and deuterium and the charged pion form factor was determined from the hydrogen cross sections. The longitudinal piece of the cross section was extracted using the Rosenbluth separation method, which requires measurements of the lab cross section at two values of the virtual photon polarizations, $\epsilon$. The longitudinal cross section was then compared to the values calculated from the VGL/Regge model and the pion form factor was extracted from 
a one-parameter fit.

The $F_{\pi}$ data point at $Q^{2}=1.60(\mathrm{GeV} / \mathrm{c})^{2}$ is in good agreement with the previous data taken during $\mathrm{F}_{\pi}-1$ indicating a small uncertainty in the extraction method. The extracted values for $F_{\pi}$ show a $1 \sigma$ deviation from the monopole form at $Q^{2}=2.45$ $(\mathrm{GeV} / \mathrm{c})^{2}$. Several calculations provide a good description of the measured values up to $Q^{2}=1.60(\mathrm{GeV} / \mathrm{c})^{2}$. The $\mathrm{F}_{\pi^{-}}-2$ data point at $Q^{2}=2.45(\mathrm{GeV} / \mathrm{c})^{2}$ deviates slightly from the Dyson Schwinger calculation by Maris and Tandy. 
Appendix A

\section{Target Density - Luminosity Scan Runs}

The size of the electron beam typically used in experiments at JLab is relatively small. While small beam dimensions ensure energy resolution and thus high quality beam properties they can result in measurable modifications to the average cryogenic target density. Localized target density fluctuations are induced by large power deposition per unit area in the target, which in turn can result in localized boiling of the target fluid. To reduce the localized target heating effects, the beam was deflected by a time varying magnetic field generated by magnets located approximately 20 meters upstream of the target. The result of the beam rastering is to distribute the beam intensity over a small area rather than localizing it at any one point on the target.

The effect of the beam current on local target density can be measured by comparing the yields at fixed kinematics and varying beam current. During $\mathrm{F}_{\pi^{-}}-2$ the current dependence of the yields was studied using so-called luminosity scans. For each luminosity scan, runs were taken for beam currents between 10 and 90 uA for each target (Hydrogen, Deuterium). The kinematics (see Table A.1) as determined by the spectrometer angles and momenta were set for elastic or deep inelastic electron scattering. To check that the analysis properly accounted for rate dependent effects, additional data were taken with a solid carbon target. For this target there should be no density reduction effects at the given beam currents (boiling point of carbon: $4554 \mathrm{~K}$ ). In particular, the luminosity scan on carbon provides a way to test the validity of the charge measurement and efficiency and 


\begin{tabular}{|l|c|c|}
\hline $\begin{array}{l}E_{\text {beam }} \\
(\mathrm{GeV})\end{array}$ & $\begin{array}{c}P_{e} \\
(\mathrm{GeV} / \mathrm{c})\end{array}$ & $\begin{array}{c}\theta_{e} \\
(\mathrm{deg})\end{array}$ \\
\hline \hline 4.210 & 3.00 & 12.00 \\
3.779 & 2.00 & 20.00 \\
3.779 (carbon) & 3.50 & 10.54 \\
4.709 & 4.05 & 10.57 \\
\hline
\end{tabular}

Table A.1: Kinematic settings for the $F_{\pi}$-2 luminosity scans.

deadtime corrections over an extended dynamic range of current and rate. The luminosity scans also include carbon data taken at fixed high beam current and varying computer deadtime to verify in detail the calculation of computer deadtime from experimental data.

The luminosity scan data were analyzed for each target using two different methods. The first method is a scaler based analysis, which makes use of only the raw counting rates while the second method uses all valid particle events based on valid drift chamber information.

The scaler based analysis entails the study of the raw HMS and SOS pretrigger signals. The trigger configuration was set up for electron detection in both spectrometers for all luminosity scans. Particle identification was implemented in the trigger, so that a pretrigger was generated whenever a signal from either the upper or the lower leg of the trigger logic (see diagram 2.16) was present (ELREAL). The charge normalized yields were corrected for electronic deadtime only and calculated for each beam current. The current dependent density changes were then determined from a comparison of the charge normalized pretrigger yields as a function of beam current. Unfortunately, the scaler based analyses are not very reliable at high rates $(>100 \mathrm{KHz})$ into the spectrometer due to background contributions 
from processes other than the process of interest, or due to gain shifts in the trigger thresholds.

The tracking based study entails the analysis of particle tracks including particle identification and loose cuts on the spectrometer acceptance. Therefore, tracking based analyses allow for better background rejection like background from reaction mechanisms other than the one of interest. The tracking based charge normalized yields were corrected for tracking efficiency, computer and electronic deadtimes and calculated as a function of beam current. The current dependence was then determined from these yields for HMS and SOS separately. Comparing the resulting fit to the current dependence from both spectrometers provides a semi-independent consistency check of the measurement.

The luminosity scan analysis was complicated by high particle rates into the spectrometers and uncertainties in the calibration of the beam current monitors. In particular, the interpretation of pretrigger yields that rely on calorimeter signals is difficult due to multiple particle events. The uncertainty in the beam current monitor $(\mathrm{BCM})$ calibration can be attributed to relative drifts in the zero-offsets, which can be on the order of $0.2 \mu \mathrm{A}$. Such fluctuations lead to offsets in the local BCM calibrations relative to a global one. Offsets in the beam current monitor calibration affect the current dependence of the normalized yield. For a given beam current, the charge used in the normalization of the yield is calculated from the global BCM calibration for an experiment. Since the charge is directly proportional to the current, an offset in the BCM calibration results in a constant offset in the measured charge. The true measured charge taking into account offsets in the BCM 
calibration can be written,

$$
Q^{\prime}=Q \cdot\left(1+\frac{d I_{\text {beam }}}{I_{\text {beam }}}\right)
$$

where $Q$ is the charge calculated from the global BCM calibration, $I_{\text {beam }}$ and $d I_{\text {beam }}$ denote the beam current and associated uncertainty, and $Q^{\prime}$ is the measured charge corrected for the uncertainty in beam current. Note that the offset in the measured charge is different at each beam current and has a larger impact at low currents. For example, a current offset of $200 \mathrm{nA}$ results in a $0.28 \%$ charge error at $100 \mu \mathrm{A}$, but becomes a $1 \%$ effect at $20 \mu \mathrm{A}$. As a result, the observed current dependence of the charge normalized yield will be affected. Ideally, one could measure this offset and apply it as an additional correction to the yield.

In this analysis, the uncertainty in the beam current offset was estimated from the global current residual determined from BCM2. The deviation from the mean current offsets from all beam current monitor calibrations was calculated at varying beam currents. To this end, a local BCM calibration was performed from data taken in the immediate vicinity of a set of luminosity scan runs. The charge normalized yields were then fit as a function of beam current and the fit quality was determined. Then, a BCM offset was applied, the modified charge was calculated and the resulting current dependence was fit. This procedure was performed for HMS and SOS tracks. The BCM offset for a particular target was taken at the minimum of the average $\chi^{2}$ determined for each data set. The BCM offset resulting from this procedure was $200 \mathrm{nA}$ for both hydrogen and deuterium. 
The contribution of the target cell walls to the normalized yield was estimated from dummy target data taken at the luminosity scan kinematics. The cell wall contribution is independent of beam current and results in a global modification of the observed current dependence of the normalized yield. In the tracking based analysis, the contribution of the cell walls is relatively small.

The normalized yields for the scaler and tracking based analyses for HMS and SOS as a function of beam current are shown in Figure A.1. The BCM offset obtained from the $\chi^{2}$ minimization described above has been applied.

During $\mathrm{F}_{\pi}-2$, luminosity data on the carbon target were taken at different rates. For the first current scan, data were taken at relatively low rates, not exceeding 200 KHz. In subsequent scans the rates were increased to match the ones obtained with the cryotargets. The result for the low rate carbon scan is shown in Figure A.3 and the one for the high rate scan is shown in Figure A.5. While no measureable current dependence can be observed at low rates, the carbon scan at high rates suggests a $1 \%$ effect at rates of $600 \mathrm{KHz}$ at $100 \mu \mathrm{A}$. The results for hydrogen and deuterium indicate no significant current dependence and no correction factor for target density modifications was applied in this analysis. 

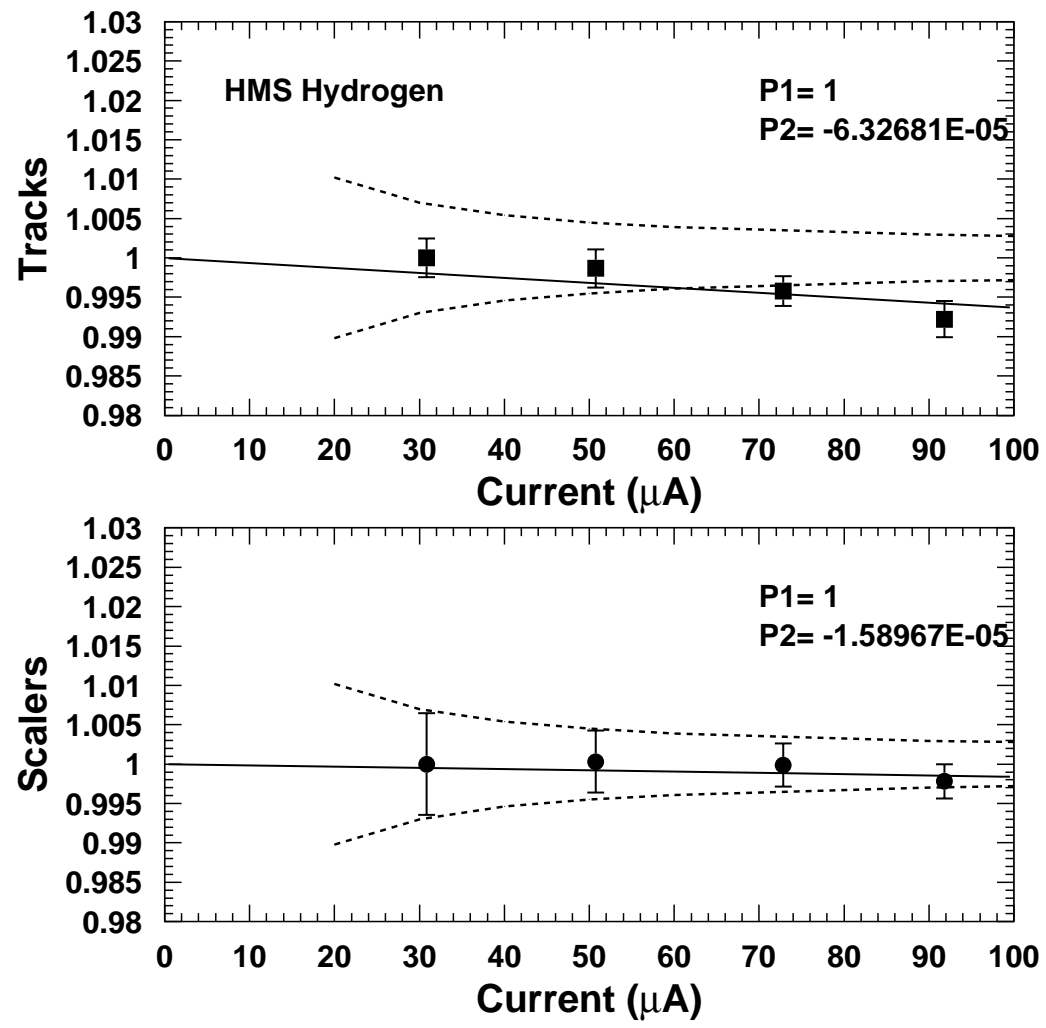

Figure A.1: Charge normalized yield as a function of beam current from the hydrogen target a measured in the HMS. The data points are normalized so that the value of the line fit to the data is one at zero beam current. The error bars are statistical only. The dashed lines denote the uncertainty in the BCM calibration as a function of beam current. 

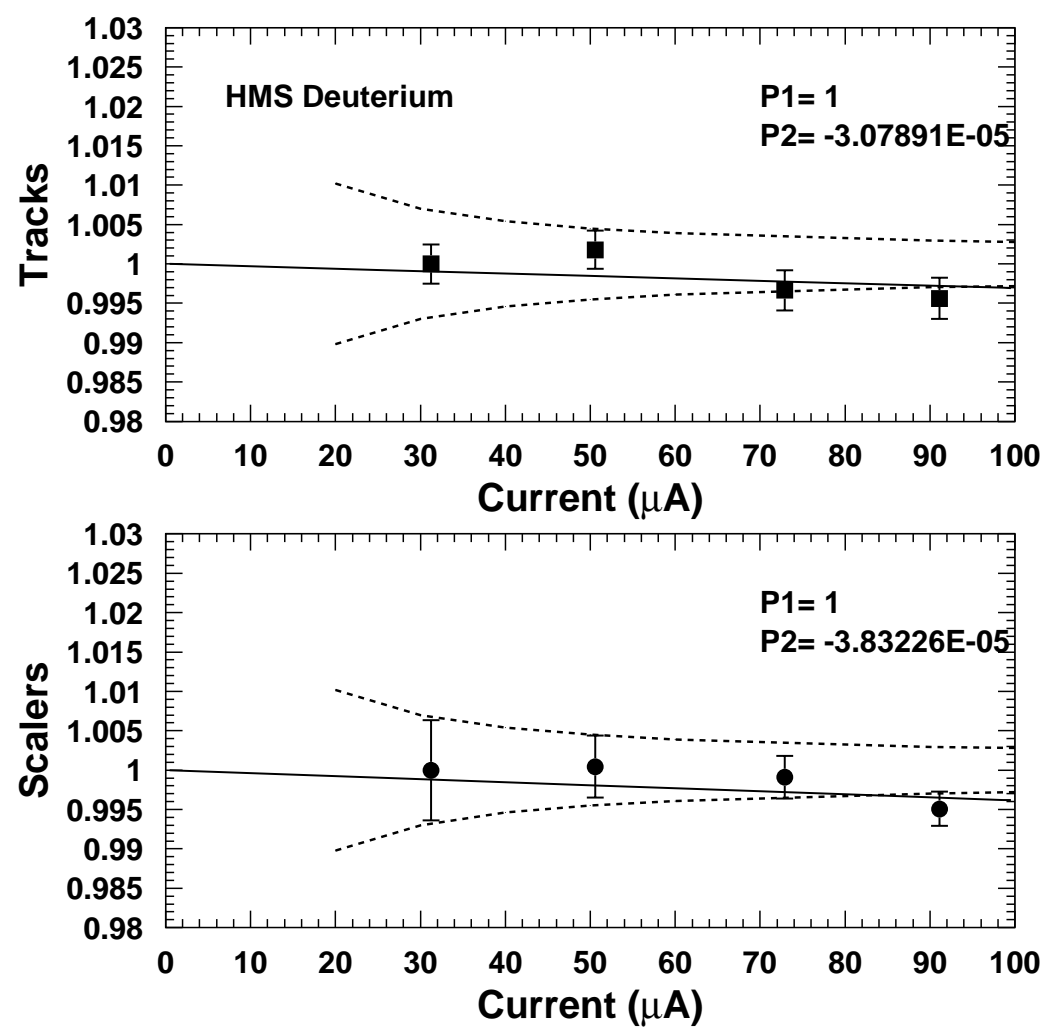

Figure A.2: Charge normalized yield as a function of beam current from the deuterium target a measured in the HMS. The data points are normalized as described in Figure A.1. 

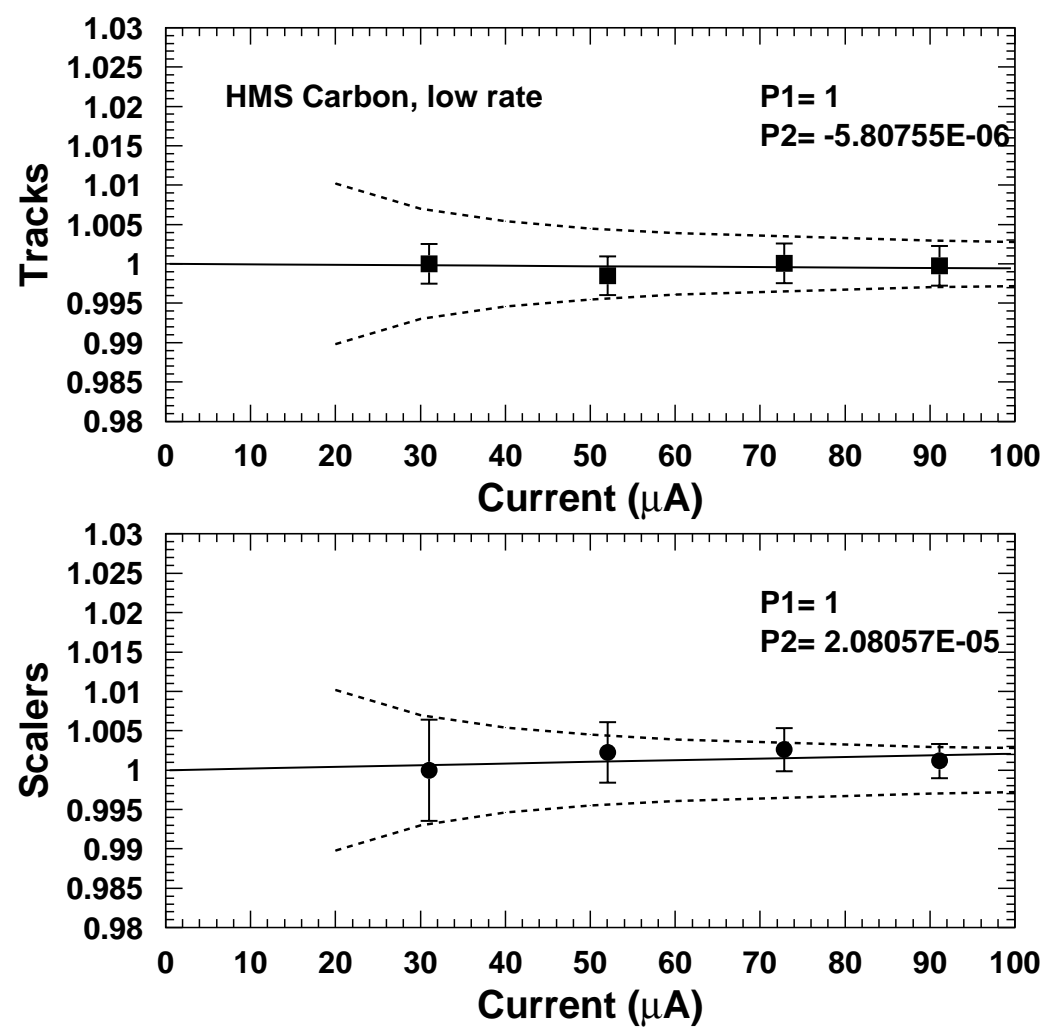

Figure A.3: Charge normalized yield as a function of beam current from the solid carbon target a measured in the HMS. The data points are normalized as described in Figure A.1. 

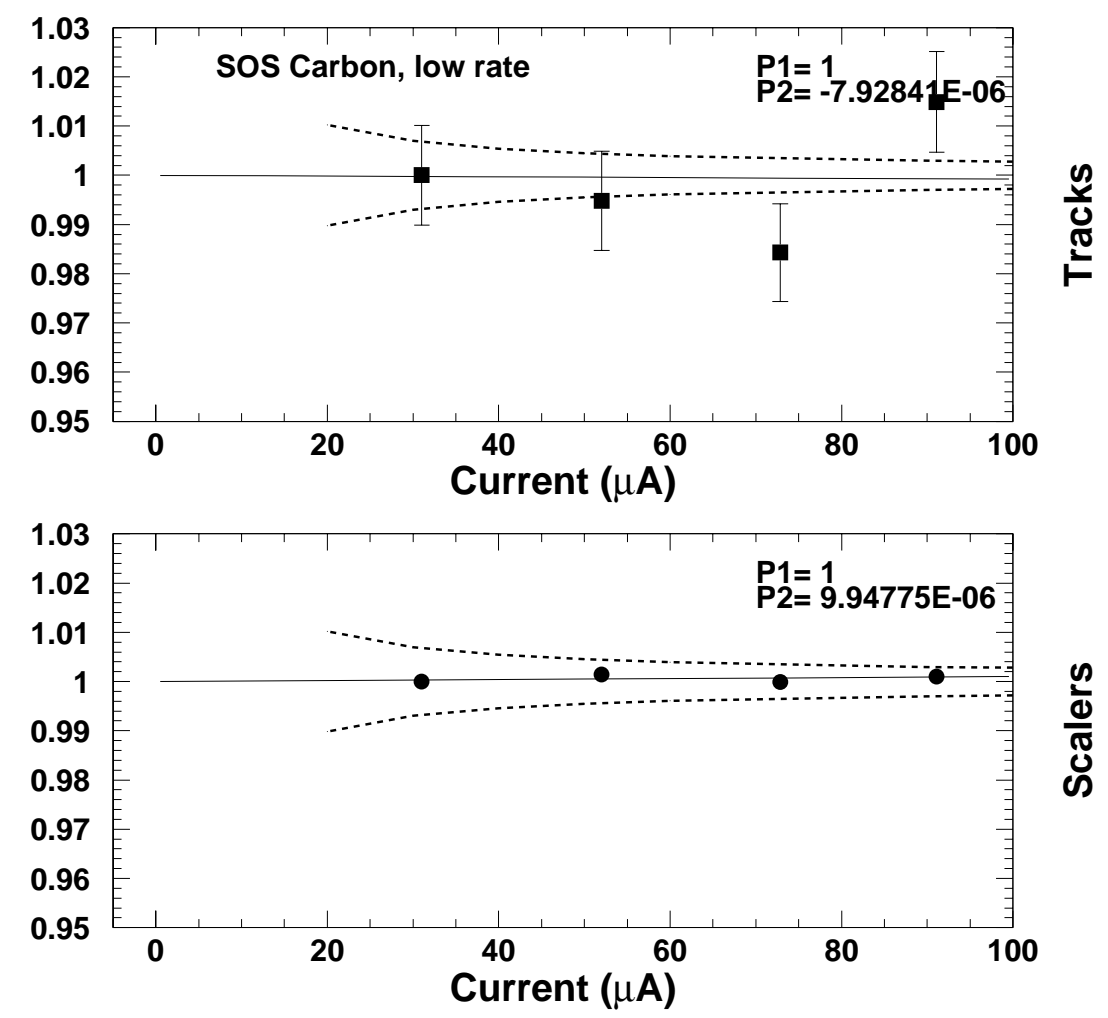

Figure A.4: Charge normalized yield as a function of beam current from the solid carbon target a measured in the HMS. The data points are normalized as described in Figure A.1. 

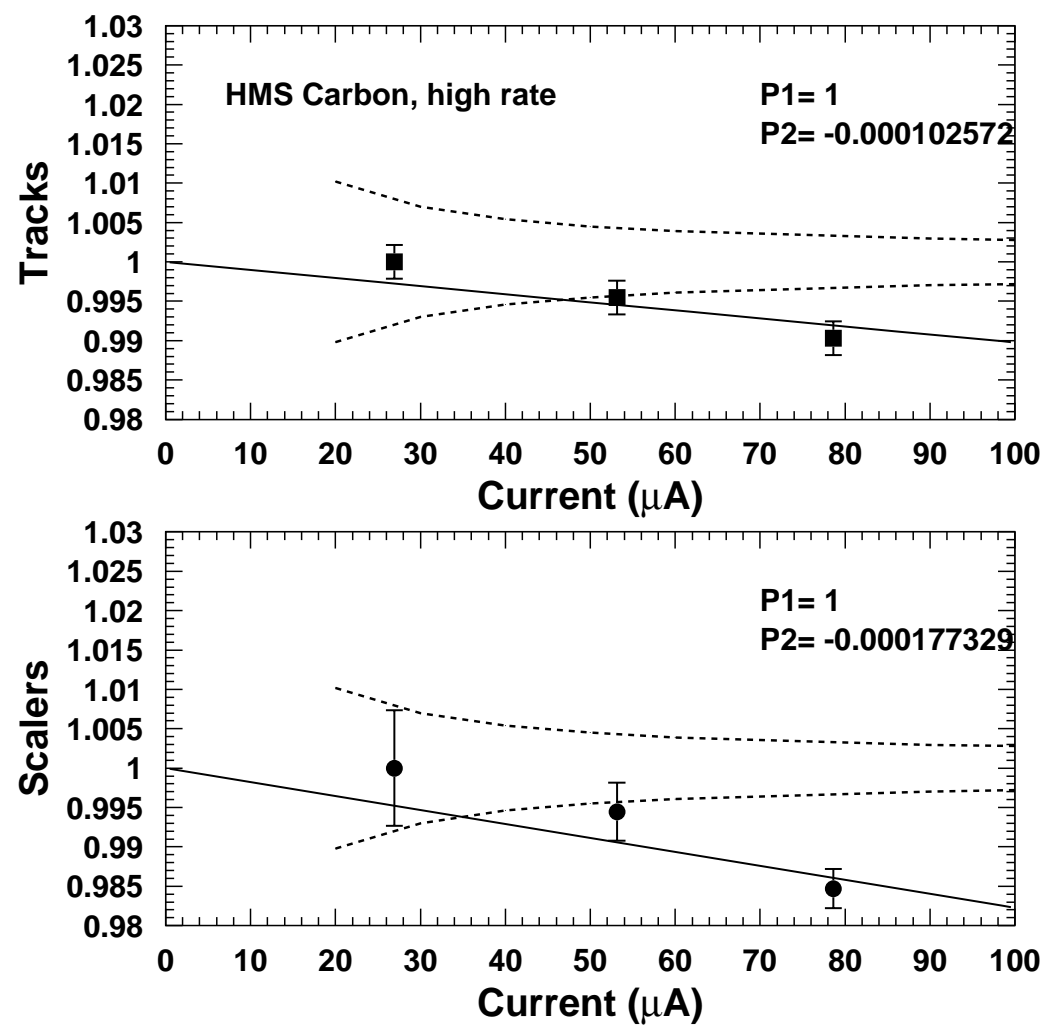

Figure A.5: Charge normalized yield as a function of beam current from the solid carbon target a measured in the HMS. The data points are normalized as described in Figure A.1. 
Appendix B

\section{Target - Thermal Contraction}

Standard materials change size with changes in temperature and over small regions of such changes the relative change in size is proportional to the temperature change with proportionality constant given by the Coefficient of Thermal Expansion. In a typical experiment the target cell length is measured at room temperature $(293 \mathrm{~K})$ whereas the operating temperature is on the order of 5 -22K. The effect of thermal contraction on the target length has to be taken into account at operating temperatures. Note that the thermal expansion coefficient is very dependent on the detailed composition of the material

The type of Aluminum used for the target cell is Al7075 an aluminum/zinc alloy (meaning high strength - other elements included of significant fraction are Mn and $\mathrm{Cu}$ ). The dummy target material is Al6061-T6, another high strength aluminum alloy. The average thermal linear expansion coefficents for Al7075 and Al6061 are listed in Table B.1. Note however that either type of Aluminum becomes significanlty non-linear at very low temperatures.

To estimate the contraction of the target cell, consider a circular geometry with circumference given by the standard form $2 \pi R$. Denote the total length of

\begin{tabular}{||l|c|}
\hline Material & $\begin{array}{c}\text { CTE } \\
\left(\mu \mathrm{m} / \mathrm{m}^{*} \mathrm{C}\right)\end{array}$ \\
\hline \hline Al 7075 & $23.4 \pm 0.3$ \\
Al 6061 & $23.6 \pm 0.3$ \\
Al 6061-T6 & $24.3 \pm 0.3$ \\
\hline
\end{tabular}

Table B.1: Material properties for Aluminium alloys used in Hall C target cell walls 
the circumference by $L$. The relative change in diameter is then equivalent to the relative change in circumference.

$$
\begin{aligned}
L & =2 \pi R \\
\delta L & =2 \pi \delta R \\
\frac{\delta L}{L} & =2\left(\frac{\delta R}{2}\right) \pi R=\frac{\delta R}{R}
\end{aligned}
$$

Using the coefficient of thermal expansion above and assuming a final temperature for hydrogen target operating temperature and an initial target radius of $4.018 \mathrm{~cm}:$

$$
L_{293 K}-\frac{L_{19 K}}{L_{293 K}}=\left(\frac{23.4 \mu m}{m * K}\right) *\left(10^{-6} \frac{m}{\mu m}\right) *(293 K-19 K) *(0.04018 m)
$$

The results for the relative change in target length as a function of temperature are shown in Figure B.1. The dashed curve is a calculation from equation B.2 using a linear thermal coefficient. The solid curve denotes a parameterization for Al6061-T6 for data between 4 and $300 \mathrm{~K}$ [115]. The data show a non-linear behavior at low temperatures and approach a constant value below a particular critical temperature. The behavior at low temperature is similar for all Aluminum alloys, thus requiring one to use a constant coefficient in calculations of thermal contraction. Assuming uncertainties in temperature $(d T=200 \mathrm{mK})$, cell radius $(d R=0.0001 \mathrm{~m})$ and thermal expansion coefficient of $(d \alpha=0.3-0.4 \mathrm{um} / \mathrm{m} * \mathrm{~K})$ results in an uncertainty of $\approx 0.2 \%$. 


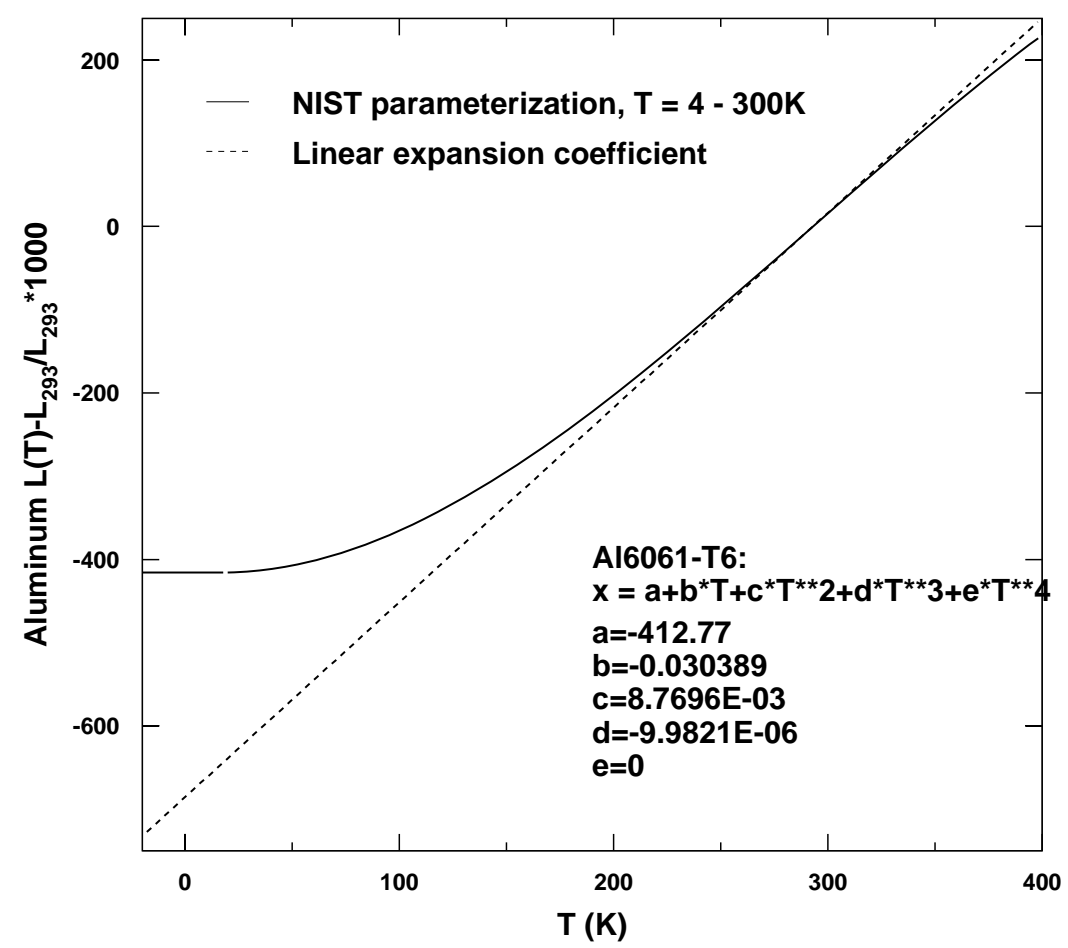

Figure B.1: Representative example of Aluminum thermal contraction for Al6061T6. The solid curve denotes a parameterization to data between 4 and $300 \mathrm{~K}$, while the dashed line is a calculation assuming a linear Thermal Expansion Coefficient. The data deviate significantly from the linear prediction at low temperature approaching a constant value. Note that this behavior is similar for all Aluminum alloys. 


\section{BIBLIOGRAPHY}

[1] W. Melnitchouk, R. Ent and C. Keppel, Phys. Rept. 406, 127 (2005).

[2] G.R.Farrar and D.R. Jackson, Phys. Rev. Lett. 43, 246 (1979).

[3] N. Isgur and C. H. LLewellyn-Smith, Phys. Rev. Lett. 52, 1080 (1984); N. Isgur and C. H. LLewellyn-Smith, Nucl. Phys. B317, 526, (1989).

[4] S.R. Amendolia et al., Nucl. Phys. B277, 168 (1986).

[5] S.R. Amendolia et al., Phys. Lett. B138, 454 (1984).

[6] C.J.Bebek et al., Phys. Rev. D17, 1693 (1978).

[7] C.J.Bebek et al., Phys. Rev. Lett. 37, 1326 (1976); C.J.Bebek et al., Phys. Rev. D13, 25 (1976).

[8] C.J.Bebek et al., Phys. Rev. D9, 1229 (1974).

[9] R. C. E. Devenish and D. H. Lyth, Phys. Rev. D5, 47 (1972).

[10] C.E. Carlson and J. Milana, Phys. Rev. Lett. 65, 246 (1979).

[11] M. Vanderhaeghen, M. Guidal and J.-M. Laget, Phys. Rev. C57, 1454 (1998); Nucl. Phys. A627 645 (1997).

[12] F. A. Berends, Phys. Rev. D1, 2590 (1970).

[13] F. Gutbrod and G. Kramer, Nucl. Phys. B49, 461 (1972). 
[14] P.Brauel et al., Phys. Lett. B69, 253 (1977); P.Brauel et al., Z. Phys. C3, 101 (1979).

[15] H. Ackermann et al., Nucl. Phys. B137, 294 (1978).

[16] J. Volmer et al., Phys. Rev. Lett 86, 1713 (2001).

[17] H.P. Blok, G.M. Huber and D.J. Mack, nucl-ex/0208011 (2004).

[18] D.J. Mack, H.P. Blok and G. M. Huber, E96-00\%, extension proposal to experiment E93-026.

[19] G. P. Lepage and S. J. Brodsky, Phys. Lett. B87, 359 (1979).

[20] A. V. Efremov and A. V. Radyushkin, Phys. Lett. B94, 245 (1980); A. V. Efremov and A. V. Radyushkin, Theor. Math. Phys. B42, 97 (1980).

[21] F.M. Dittes and A. V. Radyushkin, Phys. Lett. 134B, 359 (1984).

[22] F. Cardarelli et al., Phys. Lett. B332, 1 (1994); Phys. Lett. B357 267 (1995).

[23] V. A. Nesterenko and A. V. Radyushkin, Phys. Lett. B115, 410 (1982).

[24] P. Maris and P. C. Tandy, Phys. Rev. C62, 204 (2000).

[25] R. Jakob and P. Kroll, Phys. Lett. B315, 463 (1993).

[26] A.P. Bakulev, K. Passek-Kumericki, W. Schroers and N.G. Stefanis, Phys. Rev. D70, 033014 (2004).

[27] A.P. Bakulev and A.V. Radyushkin, Phys. Lett. B271, 223 (1991). 
[28] J. Botts and G. Sterman, Nucl. Phys. B325, 62 (1989); H. N. Li and G. Sterman, Nucl. Phys. B381, 129 (1992).

[29] G. Sterman and P. Stoler, Ann. Rev. Nucl. Part. Sci 47, 193 (1997).

[30] R. Jakob et al., J. Phys. G22, 45 (1996).

[31] V. M. Braun, A. Khoddjamirian and M. Maul, Phys. Rev. D61, 073004 (2000).

[32] P.O. Bowman, U.M. Heller and A.G. Williams, Phys. Rev. D66, 014505 (2002); P.O. Bowman, U.M. Heller, D.B. Leinweber and A.G. Williams, Modelling the quark propagator, hep-lat/0209129 (2002).

[33] M.S. Bhagwat, M.A. Pichowsky and C.D. Roberts and P.C. Tandy, Phys. Rev. C68, 015203 (2003).

[34] M.S. Bhagwat and P.C. Tandy, Phys. Rev. D70, 094039 (2004).

[35] J. Van der Heide, J. H. Koch and E. Laermann, Phys. Rev. D69, 094511 (2004).

[36] J. Van der Heide, M. Lutterot, J. H. Koch and E. Laermann, Phys. Lett. B566, $131(2003)$.

[37] F. D. R. Bonnet et al., hep-lat/0411028, (2004).

[38] G. Martinelli, C. T. Sachradajda, Nucl. Phys. B306, 365 (1988).

[39] J.W. Moffat and V. G. Snell, Phys. Rev. D4, 1452 (1970).

[40] J. S. Conway et al., Phys. Rev. D39, 92 (1989). 
[41] W. Melnitchouk, Eur. Phys. J. A17, 223 (2003).

[42] G.B. West, Phys. Lett. 24, 1206 (1970); Phys. Rev. D14 732 (1976).

[43] S.D. Drell and T.-M. Yan, Phys. Rev. Lett. 24, 131 (1970).

[44] J. S. Conway, Ph.D. thesis, University of Chicago, Fermilab-thesis-1987-16.

[45] K. Wijesooriya, P. E. Reimer and R. J. Holt, Phys. Rev. C72, 065203 (2005).

[46] I. Cloet, private communication.

[47] O. C. Jacobs and L. S. Kisslinger, Phys. Lett. B243, 323 (1990).

[48] P. Maris and P. C. Tandy, Phys. Rev. C58, 3659 (1998); P. Maris and C.R. Roberts, Phys. Rev. C58 3659 (1998).

[49] M.S. Bhagwat, Aspects Of Non-Perturbative QCD For Hadron Physics, Ph.D Thesis, Kent State University, (2005).

[50] P. L. Chung, F. Coester and W. N. Polyzou, Phys. Lett. B205, 545 (1988).

[51] C.-W. Hwang, Phys. Rev. D64, 034001 (2001).

[52] A. F. Krutov and V. E. Troitsky, Eur. Phys. J. C20, 71 (2001).

[53] F. Coester and W. N. Polyzou, nucl-th/0405082, (2004).

[54] B.V. Geshkenbein, Phys. Rev. D61, 033009 (2000).

[55] D. Gaskell, Longitudinal Electroproduction of Charged Pions on Hydrogen, Deuterium, and Helium-3, Ph.D. Thesis, Oregon State University, (2001). 
[56] J. Volmer, The Pion Charge Form Factor via Pion Electroproduction on the Proton, Ph.D. Thesis, Vrije Universiteit te Amsterdam (2000).

[57] L. N. Hand, Phys. Rev. 129, 1834-1846 (1962).

[58] I. T. Obukhovsky, D. Fedorov, A. Faessler, T. Gutsche and V. E. Lyubovitskij, hep-ph/0506319 (2004).

[59] D.J. Mack, H.P. Blok, G.M. Huber, E01-004, extension proposal to experiment E93-021.

[60] C. E. Reece, Operating Experience With Superconducting Cavities at Jefferson Lab, Invited Contribution to the Eighth Workshop on RF Superconductivity, Abano Terme, Italy,(1997).

[61] R. Legg, Operating Experience at CEBAF, JLab Technical Note PR96-013, (unpublished).

[62] I. Niculescu, Shower Counter and Cerenkov Efficiencies, JLab Hall C Internal Report, (1997, unpublished).

[63] C. Yan et al., Nucl. Inst. and Meth. A365, 261 (1995).

[64] M.K. Jones, private communication.

[65] C.Yan, R.Carlini and D. Neuffer,Beam Energy Measurement Using the Arc Beam Line as a Spectrometer, CEBAF-PR-93-004, (1993).

[66] K. Unser, Proceedings of the Accelerator Instrumentation Workshop, edited by W. Barry and P. Kloeppel, A.I.P. Conf. Proc. 252, 266 (1992). 
[67] D. Mack, Mu metal shielding measurements, http://hallcweb.jlab.org/hclog/0307_archive/030708103928.html.

[68] D. Mack, Mu metal shielding, http://hallcweb.jlab.org/hclog/030\%_archive/030708111738.html, http://hallcweb.jlab.org/hclog/0307_archive/030708113704.html.

[69] R. Wojcik and C. Yan, et al., Nucl. Inst. and Meth. A484, 690 (2002).

[70] C. Yan et al., Nucl. Inst. and Meth. A365, 46 (1995).

[71] D. Gaskell, private communication.

[72] J. Dunne, Cryo and Dummy Target Information, JLab Hall C Internal Report, (unpublished).

[73] D.G. Meekins, Ph.D. thesis, The College of William and Mary, 1999.

[74] B. Terburg, , Ph.D. Thesis, University of Illinois (1999).

[75] D.G. Meekins, private communication.

[76] Experiment Safety Assessment Document for the Hall C Base Equipment, Jlab Hall C document (2005).

[77] B. Clasie, private communication.

[78] J.R. Arrington, Inclusive Electron Scattering From Nuclei at $x>1$ and High $Q^{2}$, Ph.D. Thesis, California Institute of Technology, (1998).

[79] R. Asaturyan et al., Nucl. Instrum. Meth. A548, 364-374 (2005). 
[80] D. Abbott et al., Proceedings of the 1995 IEEE Conference on Real-Time Computing Applications in Nuclear, Particle and Plasma Physics, 147 (1995).

[81] V. Tvaskis, , Ph.D. Thesis, Vrije Universiteit te Amsterdam (2004).

[82] H. P. Blok, private communication.

[83] M. Berz, Technical Report MSUCL-977, Michigan State University (1995) unpublished.

[84] K.Assamagan, D.Dutta and P. Welch,Hall C Matrix Element Optimization Package (CMOP), Jefferson Lab Hall C Internal Report (unpublished).

[85] D.Dutta, , Ph.D. Thesis, Northwestern University, (1999).

[86] M.K. Jones, Hall C User Howto - Optimize Optics Matrix Elements, Hall C Internal Document (unpublished).

[87] H. P. Blok, Beam centering and effect of vertical beam offsets, http://hallcweb.jlab.org/hclog/0307_archive/030713122338.html.

[88] J. Volmer and H.P. Blok, Usage of Heepcheck, Jefferson Lab Hall C Internal Report, (unpublished).

[89] M. E. Christy et al., Phys. Rev. C70, 015206 (2004).

[90] M. E. Christy, private communication.

[91] W.R. Leo - MINUIT - Techniques for Nuclear and Particle Physics Experiments, (Springer Verlag, Berlin, 1994). 
[92] R.M. Mohring, , Ph.D. Thesis, University of Maryland, (1999).

[93] M. J. Longo and B. J. Moyer, Phys. Rev. 125, 701 (1962).

[94] N. C. R. Makins, Ph.D. thesis, Massachussetts Institute of Technology (1994).

[95] COSY INFINITY, Proceedings of the 1991 Particle Accelerator Conference, see, http://bt.nscl.msu.edu/cosy/index.htm.

[96] D.H. Potterveld, private communication.

[97] L. Alvarez-Gaume et al., Phys. Lett. B592, 1 (2004).

[98] S. Lebedev, utiLandau PHENIX utility, www.phenix.bnl.gov/WWW/software/luxor/uti/mod 1998.

[99] J. Volmer, Calculation of Energy Losses in Hall C's Replay Engine, Jefferson Lab Hall C Internal Report, (unpublished).

[100] R. Ent et al., Phys. Rev. C64, (2001).

[101] L. M. Mo and Y. S. Tsai, Rev. Mod. Phys 41, 205 (1969).

[102] N.C.R. Makins, , Ph.D. Thesis, Massachussetts Institute of Technology, (1994).

[103] P.G. Blunden, W. Melnitchouk and J.A. Tjon, Phys.Rev. C72, 034612, (2005).

[104] A.V. Afanasev et al., Phys.Rev. D72, 013008, (2005).

[105] J.R. Arrington, Phys. Rev. C69, 022201 (2004). 
[106] P.E. Bosted, Phys. Rev. C51, 409 (1995).

[107] D.van Westrum, , Ph.D. Thesis, University of Colorado, (1998).

[108] D.van Westrum, Measurement and Estimate of Proton Absorption in Hall C Spectrometers, Jefferson Lab Hall C Internal Report, (unpublished).

[109] F. James - MINUIT - Function Minimization and Error Analysis, Reference Manual.

[110] P.R. Bevington - Data Reduction and Error Analysis for the Physical Sciences, (McGraw-Hill, Inc., 1992).

[111] Y. Yamaguchi, Phys. Rev. 95, 1628 (1954).

[112] J. Dubach, W. M. Kloet and R.R. Silbar, Phys. Rev. C33, 373 (1986).

[113] C. Roberts, private communication.

[114] Hall-C Collaboration, Hall C 12 GeV Upgrade Conceptual Design Report, Jlab Hall C document (2002).

[115] National Institute of Standards and Technology, Cryogenic Technologies Group, http://cryogenics.nist.gov/NewFiles/6061_T6_Aluminum.html. 